volume 7

ISSUE 4

2019 giugno

Editor in Chief Paola Gnerre

Supervisor Editor Roberto Nardi

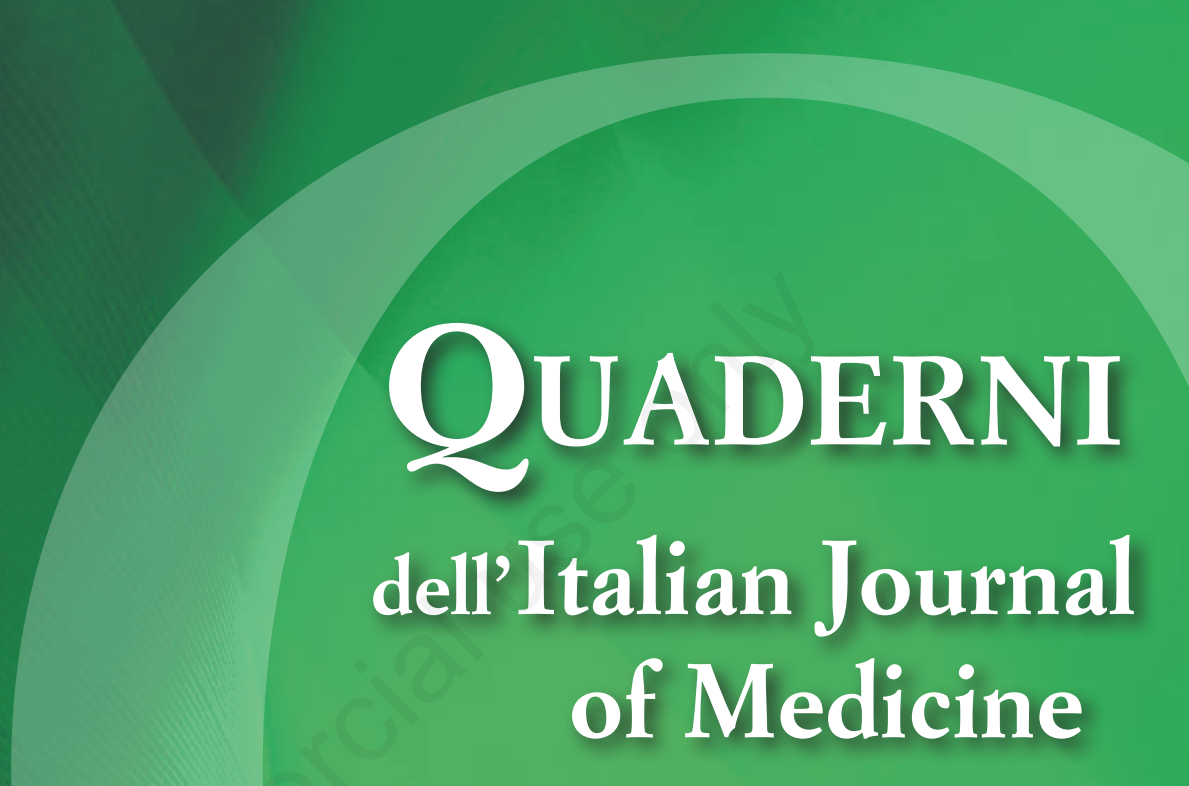

A Joumal of Hospital

and Internal Medicine

The official journal of the Federation of Associations of Hospital Doctors on Internal Medicine (FADOI)

\title{
I diritti negati agli anziani: quali implicazioni per la salute?
}

Guest Editors: R. Nardi, P. Gnerre, A. Salsi, A. Fontanella, D. Manfellotto 
PRESIDENTE ELETTO

Dario Manfellotto, Roma, Italy

PAST PRESIDENT

Mauro Campanini, Novara, Italy

SEGRETARIO

Micaela La Regina, La Spezia, Italy

SEGRETARIO VICARIO

Andrea Montagnani, Grosseto, Italy

\section{STAFF DI SEGRETERIA}

\begin{tabular}{ll}
\hline Comunicazione & Claudia Tieri, Bari, Italy \\
Ricerca & Roberta Re, Novara, Italy \\
Formazione & Maurizia Gambacorta, Todi $(P G)$, Italy
\end{tabular}

TESORIERE

David Terracina, Roma, Italy

\section{STAFF DI TESORERIA}

Francesco D'Amore, Roma, Italy

PRESIDENTE FONDAZIONE FADOI

Mauro Campanini, Novara, Italy

COORDINATORE

Giuseppe Augello, Canicatti ( $A G)$, Italy

\section{DIPARTIMENTO PER LA RICERCA CLINICA FADOI}

Direttore Francesco Dentali, Varese, Italy

Supervisor per la Ricerca Giancarlo Agnelli, Perugia, Italy

DIPARTIMENTO PER LA FORMAZIONE

E AGGIORNAMENTO

Direttore Mauro Silingardi, Bologna, Italy

COORDINAMENTO FORMAZIONE

AREA CENTRO-NORD

Francesco Orlandini, La Spezia, Italy

COORDINAMENTO FORMAZIONE

AREA CENTRO-SUD

Generoso Uomo, Napoli, Italy

COORDINATORE COMMISSIONE GIOVANI

Flavio Tangianu, Oristano, Italy

MEMBRO FISM E RESPONSABILE

PER L'INNOVAZIONE IN MEDICINA INTERNA

Antonino Mazzone, Legnano (MI), Italy

ITALIAN JOURNAL OF MEDICINE

Editor in Chief Giorgio Vescovo, Padova, Italy
RESPONSABILE DEI

QUADERNI DELL'ITALIAN JOURNAL OF MEDICINE

Paola Gnerre, Savona, Italy

SUPERVISOR EDITOR DEI

QUADERNI DELL'ITALIAN JOURNAL OF MEDICINE

Roberto Nardi, Bologna, Italy

DELEGATO FADOI ITALIAN STROKE ORGANIZATION

E CONSULTA CARDIOVASCOLARE

Michele Stornello, Siracusa, Italy

\section{RAPPORTI CON EFIM}

Antonio Brucato, Bergamo, Italy

Gualberto Gussoni, Milano, Italy

Ombretta Para, Firenze, Italy

Giorgio Vescovo, Padova, Italy

RESPONSABILE SITO NAZIONALE

Salvatore Lenti, Arezzo, Italy

RESPONSABILE SISTEMA GESTIONE QUALITÀ

Franco Berti, Roma, Italy

RESPONSABILE CLINICAL COMPETENCE

Antonino Mazzone, Legnano (MI), Italy

RESPONSABILE AREA ECOGRAFIA

Francesco Cipollini, Ascoli Piceno, Italy

Marcello Romano, Catania, Italy

\section{RESPONSABILE MEDICINA DI GENERE}

Cecilia Politi, Isernia, Italy

\section{RESPONSABILE AREA NUTRIZIONE}

Roberto Risicato, Siracusa, Italy

Massimo Rondana, Pordenone, Italy

Luciano Tramontano, Praia a Mare (CS), Italy

RESPONSABILE AREA DI CLINICAL GOVERNANCE

Giovanni Iosa, Cesenatico ( $F C$ ), Italy

Stefano De Carli, Udine, Italy

\section{AREA SLOW MEDICINE}

Roberto Frediani, Chieri (TO), Italy

Luigi Lusiani, Castelfranco Veneto (TV), Italy

\section{AREA DOLORE}

Domenico Panuccio, Bologna, Italy

Giuseppe Civardi, Piacenza, Italy

\section{AREA CRITICA IN MEDICINA INTERNA}

Carlo Nozzoli, Firenze, Italy

\section{RESPONSABILE AGGIORNA FADOI}

Giuliano Pinna, Asti, Italy 


\section{Italian Journal of Medicine}

A Journal of Hospital and Internal Medicine

\section{PRESIDENTE FONDAZIONE}

Mauro Campanini, Novara, Italy

\section{COORDINATORE}

Giuseppe Augello, Canicattì ( $A G)$, Italy

\section{DIPARTIMENTO PER LA RICERCA CLINICA FADOI}

Direttore $\quad$ Francesco Dentali, Varese, Italy

Supervisor per la Ricerca Giancarlo Agnelli, Perugia, Italy

SEGRETERIA

Grazia Panigada, Pescia (PT), Italy

DELEGATO SIF

Francesco Rossi, Napoli, Italy

DELEGATO ANÍMO

Alberto Dal Molin, Novara, Italy

RESPONSABILE AREA MALATTIE CARDIOVASCOLARI

Paolo Verdecchia, Assisi (PG), Italy

\section{STAFF AREA MALATTIE CARDIOVASCOLARI}

Cecilia Becattini, Perugia, Italy

Pierpaolo Di Micco, Napoli, Italy

Fernando Gallucci, Napoli, Italy

Alessandro Squizzato, Varese, Italy

\section{RESPONSABILE AREA MALATTIE INFETTIVE}

Ercole Concia, Verona, Italy

\section{STAFF AREA MALATTIE INFETTIVE}

Anna Maria Azzini, Verona, Italy

Gianluca Giuri, Castelnovo ne'Monti (RE), Italy

Matteo Giorgi Pierfranceschi, Piacenza, Italy

Carlo Tascini, Pisa, Italy

\section{RESPONSABILE AREA MALATTIE RESPIRATORIE}

Leonardo Fabbri, Reggio Emilia, Italy

\section{STAFF AREA MALATTIE RESPIRATORIE}

Bianca Beghè, Reggio Emilia, Italy

Gaetano Cabibbo, Modica (RG), Italy

Francesco Corradi, Firenze, Italy

Francesco Ventrella, Cerignola $(F G)$, Italy

\section{RESPONSABILE AREA MALATTIE REUMATOLOGICHE}

Carlo Salvarani, Reggio Emilia, Italy

\section{STAFF AREA MALATTIE REUMATOLOGICHE}

Paola Faggioli, Legnano (MI), Italy

Laura Morbidoni, Senigallia (AN), Italy

Nicolò Pipitone, Reggio Emilia, Italy

Tito D'Errico, Napoli, Italy

\section{RESPONSABILE AREA MALATTIE METABOLICHE}

Roberto Vettor, Padova, Italy

\section{STAFF AREA MALATTIE METABOLICHE}

Tiziana Attardo, Agrigento, Italy

Giovanni Gulli, Savigliano (CN), Italy

Ada Maffettone, Napoli, Italy
Maurizio Nizzoli, Forli, Italy

RESPONSABILE AREA ORGANIZZATIVA

IN MEDICINA INTERNA

Antonio Greco, San Giovanni Rotondo ( $F G)$, Italy

\section{STAFF AREA ORGANIZZATIVA IN MEDICINA INTERNA}

Marco Candela, Jesi (AN), Italy

Giovanni Mathieu, Pinerolo (TO), Italy

Valentino Moretti, San Daniele del Friuli (UD), Italy

Filomena Pietrantonio, Brescia, Italy

Elisa Romano, La Spezia, Italy

DIRETTORE DIPARTIMENTO PER LA FORMAZIONE

E AGGIORNAMENTO

Mauro Silingardi, Bologna, Italy

COORDINAMENTO DI AREA FORMATIVA CENTRO-NORD

Francesco Orlandini, La Spezia, Italy

COORDINAMENTO DI AREA FORMATIVA CENTRO-SUD

Generoso Uomo, Napoli, Italy

STAFF DIPARTIMENTO PER LA FORMAZIONE

E AGGIORNAMENTO

Roberto Frediani, Chieri (TO), Italy

Marco Grandi, Sassuolo (MO), Italy

\section{SEGRETERIA DIPARTIMENTO PER LA FORMAZIONE} E AGGIORNAMENTO

Luigi Magnani, Voghera (PV), Italy

\section{BOARD SCIENTIFICO}

Clelia Canale, Reggio Calabria, Italy

Fabrizio Colombo, Milano, Italy

Giuseppe De Matthaeis, Città Sant'Angelo (PE), Italy

Massimo Giusti, Torino, Italy

Luca Masotti, Cecina (LI), Italy

Andrea Montagnani, Grosseto, Italy

Nicola Mumoli, Livorno, Italy

Maurizio Ongari, Porretta Terme (BO), Italy

Ruggero Pastorelli, Colleferro (RM), Italy

Fulvio Pomero, Savigliano (CN), Italy

Roberto Risicato, Siracusa, Italy

Antonio Sacchetta, Treviso, Italy

Giancarlo Tintori, Pisa, Italy

COMMISSIONE TECNICHE E METODICHE INNOVATIVE DI FORMAZIONE E VERIFICA

Responsabile dell'Innovazione in Medicina Interna

Antonino Mazzone, Legnano (MI), Italy

STAFF

Francesco Dentali, Varese, Italy

Andrea Montagnani, Grosseto, Italy

Filippo Pieralli, Firenze, Italy

\section{DIRETTORE SCIENTIFICO FONDAZIONE FADOI}

Gualberto Gussoni, Milano, Italy 


\title{
QUADERNI - Italian Journal of Medicine
}

\author{
I DIRITTI NEGATI AGLI ANZIANI: \\ QUALI IMPLICAZIONI PER LA SALUTE? \\ Guest Editors: R. Nardi, P. Gnerre, A. Salsi, A. Fontanella, D. Manfellotto
}

RASSEGNE

Perchè gli internisti devono occuparsi dei diritti negati agli anziani? . . $\quad 1$ M. Campanini, P. Gnerre, A. Fontanella

Diritti negati, abusi, maltrattamenti e neglect nell'anziano: macrofenomeno mondiale sommerso, sostanzialmente misconosciuto o trascurato . . . . . . . . . . . . . . . . . . . . . . . . . . .

P. Calogero, A. Salsi

Il diritto al coordinamento delle cure e alla continuità assistenziale nella gestione delle malattie croniche: strumenti disponibili oggi e quali potenzialmente fruibili in futuro? . . . . . . . . . . . . . 12 F. Orlandini

La mancata pianificazione dei fabbisogni di medici e di specialisti in Italia nel Servizio Sanitario pubblico. Quali conseguenze in rapporto all'invecchiamento della popolazione? . . . . . . . . . . . . . 21

D. Montemurro, M. D'Arienzo, C. Rivetti, E. Marcante, F. Ragazzo,

P. Di Silverio, A. Rossi, C. Palermo

I rischi di un eccesso di cure negli anziani: fare di più non significa fare meglio anche nell'anziano $\ldots \ldots \ldots \ldots \ldots \ldots \ldots \ldots$ M. Bobbio

Pensionamento: ritirarsi dal lavoro, non dalla vita né da un futuro di buona salute. . . . . . . . . . . . . . . R. Nardi, D. Borioni, D. Tirotta, F. Berti, G. Pinna, S. Ehrlich, G. Pentella, P. Cosma, R. Rapetti, A. Fontanella

Gestione della depressione, inevitabile compagna del paziente anziano $\ldots \ldots \ldots \ldots \ldots \ldots \ldots \ldots \ldots \ldots \ldots \ldots \ldots$

E. Pecoraro, S. La Carrubba

La sessualità nell'anziano

V. Duretto, G. Pinna

Gli ostacoli metodologici nella comunicazione con il paziente anziano M. Felici, A. Tufi, A. Pulerà, S. Lenti

L'importanza della capacità di educare all'autogestione delle malattie croniche (self-management) per prevenire l'aggravamento e i ricoveri ripetuti non programmati. . . . . . . . . . . . . . M. La Regina, A. Greco

Le cure di fine vita negli anziani: il diritto di morire bene

L. Lusiani, C. Santini

Il programma delle otto $R$ : utopia o possibile realtà per migliorare l'assistenza agli anziani? 


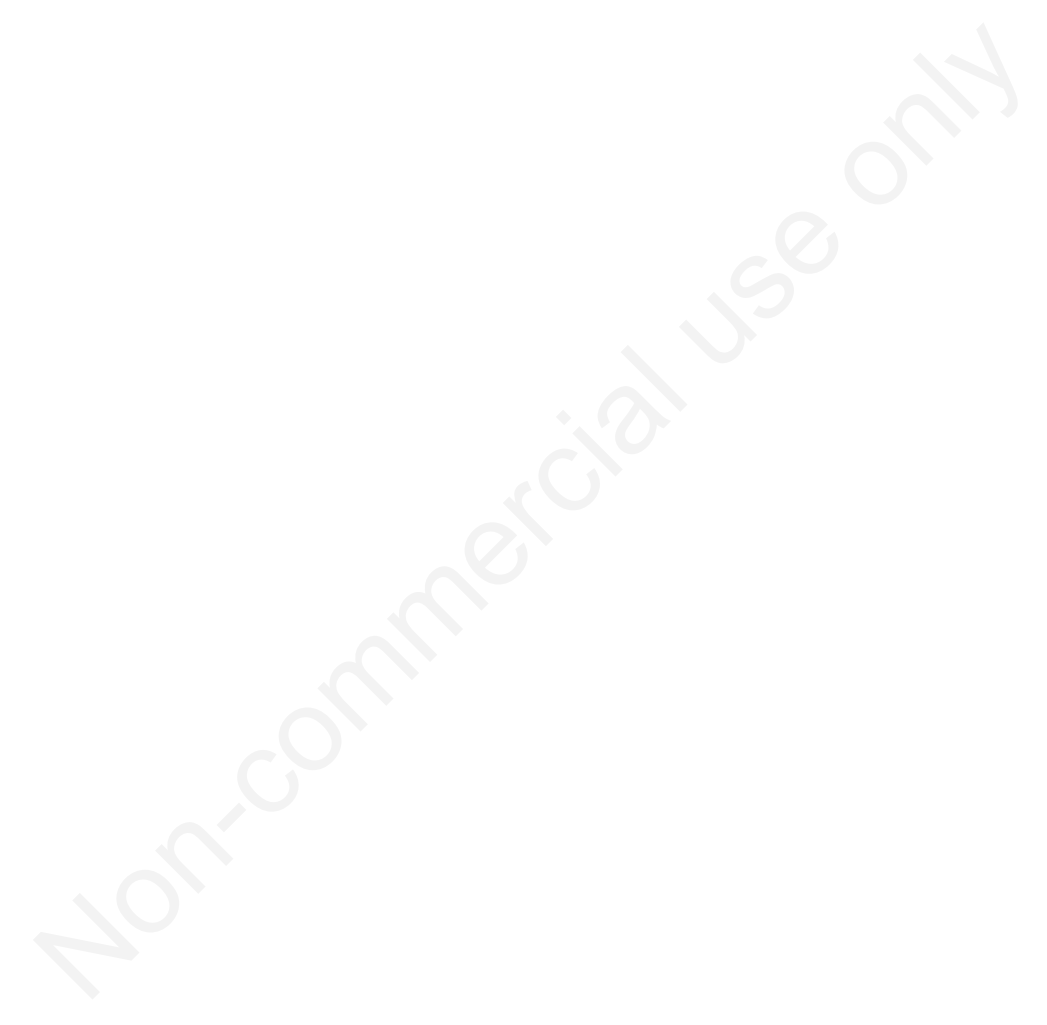




\title{
Perché gli internisti devono occuparsi dei diritti negati agli anziani?
}

\author{
Mauro Campanini, ${ }^{1}$ Paola Gnerre, ${ }^{2}$ Andrea Fontanella ${ }^{3}$ \\ ${ }^{1}$ Dipartimento di Medicina Interna 2, Ospedale Maggiore della Carità, Novara; ${ }^{2}$ Dipartimento di Medicina Interna, Ospedale \\ San Paolo, Savona; ${ }^{3}$ Presidente Nazionale FADOI, Direttore del Dipartimento di Medicina e Pronto Soccorso, Ospedale \\ Buon Consiglio Fatebenefratelli, Napoli, Italia
}

In presenza di un forte allarme sociale, provocato dal prevalere degli egoismi nazionalistici ed individuali, in periodi in cui si vedono sempre più emergere atteggiamenti di classismo e di razzismo, si afferma in modo prepotente l'esigenza che proprio lo Stato, attraverso i propri apparati, si faccia con forza garante del rispetto dei valori di fondo (dalla legalità, alla giustizia, ai diritti fondamentali) posti alla base della nostra Carta costituzionale, di cui si sono celebrati i sessanta anni dalla sua entrata in vigore avvenuta il $1^{\circ}$ gennaio del 1948. La salvaguardia della sicurezza collettiva, indispensabile per consentire a ciascun individuo di godere una condizione di vita esente da paure, nel pieno esercizio dei suoi diritti, rappresenta l'obiettivo primario cui dovranno, infatti, tendere tutte le autorità costituite, al fine di permettere lo sviluppo di una civile convivenza, in ogni parte del territorio nazionale. Laddove c'è la percezione di una negazione di alcuni valori che la nostra Costituzione sancisce vuol dire che quella Carta è ancora, in parte, un ideale da perseguire, una speranza da trasformare in realtà, un impegno di lavoro da compiere. Tutti gli individui hanno bisogno di elementari necessità senza le quali non riescono a sopravvivere in modo qualitativamente dignitoso. Dovere morale ed etico di ogni Società è far si che ogni persona abbia accesso ai bisogni primari che comprendono la nutrizione, il vestiario, la casa, i Servizi Sanitari, l'istruzione, l'annullamento dell'emarginazione, la riduzione della mortalità infantile e il miglioramento della speranza di vita.

Secondo l'Organizzazione Mondiale della Sanità

Corrispondente: Paola Gnerre, Dipartimento di Medicina Interna, Ospedale San Paolo, Savona, Italia. Tel.: +39.019.8404358 - Fax: +39.019.8404583. E-mail: pgnerre@yahoo.it

Articolo pubblicato secondo la Creative Commons Attribution NonCommercial 4.0 License (CC BY-NC 4.0).

CCopyright M. Campanini et al., 2019

Licensee PAGEPress, Italy

QUADERNI - Italian Journal of Medicine 2019; 7(4):1-3
(OMS) tutti i Servizi Sanitari devono essere strutturati per garantire ai cittadini un'assistenza sanitaria continua, una facile accessibilità ai servizi, un adeguato rifornimento di strutture e strumenti essenziali, un personale sanitario proporzionato e un coinvolgimento del paziente e/o del care-giver nelle decisioni sanitarie da prendere. ${ }^{1}$ Tutto questo diventa più difficile da attuare se si rapporta alle popolazioni più fragili come nel caso del cittadino anziano.

L'anziano è solitamente un paziente affetto da più patologie croniche incidenti contemporaneamente (comorbidità o multimorbidità), le cui esigenze assistenziali sono determinate non solo da fattori legati alle condizioni cliniche, ma anche da altri determinanti come lo status socio-familiare, ambientale e l'accessibilità alle cure. Infatti non solo vi è il problema dell'età avanzata ma vi è anche una realtà sociale mutata, nella quale la struttura della famiglia, di cui nel passato l'anziano era il nucleo fondante e coagulante, è profondamente cambiata, con una cerchia familiare sempre più ristretta, per la riduzione della natalità, e le donne che hanno abbandonato il loro ruolo tradizionale di regine della casa, per entrare di necessità nel mondo del lavoro e con i care-giver spesso anch'essi anziani e talora afflitti da patologie croniche. La questione assume un aspetto ancora più rilevante, anche sotto il profilo economico e organizzativo, di fronte alla non autosufficienza della persona anziana.

L'aumento della spettanza di vita e della sopravvivenza farà si che nel 2025 nei paesi a maggior sviluppo la percentuale di sessantenni e ultra sessantenni rappresenterà circa il 30\% della popolazione e circa 170 milioni di persone saranno affette da malattie degenerative e croniche. Un numero destinato a lievitare con il progressivo allungamento della vita, pertanto, dato il crescente numero, la gestione del paziente anziano, sia autosufficiente che non, diventerà a breve un vero e proprio problema di salute pubblica e sarà la vera sfida sanitaria del futuro. ${ }^{2}$ La problematica è talmente incipiente che anche il Consiglio d'Europa ha adottato nel 2014 una raccomandazione in tema di promozione dei diritti degli anziani, al fine di stimolare gli Stati a promuovere misure per l'anti-discriminazione, per il sostegno e l'incentivo alla 
partecipazione e all'autonomia degli anziani, per la protezione contro violenza e abuso, per l'occupazione, per la cura e l'amministrazione della giustizia. ${ }^{3} \mathrm{La}$ Raccomandazione presenta una serie di buone pratiche che gli Stati e la società civile sono chiamati a implementare, oltre che a proclamare il fatto che i diritti fondamentali, a lungo negletti e negati nei riguardi della popolazione anziana, si applicano anche ad essi su basi di eguaglianza. E nel 2017 l'OMS ha promulgato le linee guida sulla cura integrata per le persone anziane sottolineando il ruolo dell' assistenza primaria e il contributo della comunità per prevenire, rallentare o invertire i declini delle capacità fisiche e mentali tra le persone anziane. Le linee guida forniscono, quindi, indicazioni per chi si occupa di assistenza primaria per porre al centro dell'assistenza le esigenze globali delle persone anziane e non solo le malattie. I sistemi sanitari devono soddisfare i bisogni delle popolazioni più anziane, fornendo una cura integrata e focalizzata sulla persona, con maggior impatto sulla capacità funzionale e quindi maggiormente incentrata su problematiche come il dolore cronico, le difficoltà nell'udito, nella vista, nella deambulazione o nello svolgimento delle comuni attività quotidiane. Tutti gli stati, pertanto, devono sviluppare meccanismi sostenibili di finanziamento per proteggere gli anziani e le loro famiglie da oneri finanziari indebiti, organizzare i servizi per rispondere ai vari livelli di capacità delle persone anziane e alle loro esigenze e preferenze, garantire la copertura dei servizi a tutte le persone anziane specie in merito a quegli interventi atti a mantenere la capacità intrinseca e la capacità funzionale (esempio piani di valutazione e cura completi, interventi chirurgici, dispositivi, integratori alimentari funzionali, esercizi fisici multimodali e assistenza a lungo termine). In Italia, rispetto al centro e al nord Europa, il problema si è manifestato e si manifesterà in maniera ancora più evidente a seguito della elevata durata della vita media e, in particolare, del ridotto tasso di natalità. In Italia ci sono oltre 12 milioni di ultra 65 enni, 3 milioni dei quali vivono soli. La problematica dei diritti negati agli anziani per l'accesso alle cure sanitarie è quindi una problematica impellente tanto che già nel 1990 a Parma fu elaborata la Carta rivendicativa dei diritti degli anziani cronici non autosufficienti esplicitata nei 10 punti della Tabella 1.

A causa di questa evoluzione antropologica e sociologica della nostra società, la maggioranza dei nostri pazienti sono ultrasettantacinquenni, con più patologie croniche, in poli terapia, con deficit funzionali dei principali organi emuntori, con patologie metaboliche in scompenso, con patologie cardiache

Tabella 1. Carta rivendicativa dei diritti degli anziani cronici non autosufficienti.

1. L'anziano cronico non autosufficiente è un malato e, in quanto tale, ha pieno e inalienabile diritto, al pari di ogni cittadino, alle cure sanitarie, senza limiti di durata, cause o fenomenologia. In queste cure vanno comprese, ove occorrano, quelle fornite dalie strutture ospedaliere o dalle residenze sanitarie pubbliche o private

2. Non deve esistere alcuna disparità di trattamento degli anziani cronici non autosufficienti rispetto agli altri cittadini malati, in particolare per quanto riguarda l'accesso ai servizi ambulatoriali e ospedalieri e la tempestività delle cure e delle prestazioni riabilitative

3. L'anziano cronico non autosufficiente, se ospedalizzato, ha il diritto di non essere dimesso se non esistono le premesse per una sistemazione che gli assicuri tutte le cure sanitarie di cui necessita

4. È indispensabile la presenza diffusa sul territorio (quartieri, piccoli comuni, circoscrizioni) di piccole residenze sanitarie che assicurino l'assistenza curativa e riabilitativa agli anziani cronici non autosufficienti che non possono essere curati a domicilio

5. In attesa di assicurare a tutti gli anziani cronici non autosufficienti il ricovero in strutture sanitarie (ospedali, ospedalizzazione a domicilio, piccole residenze sanitarie, ecc.) possono essere utilizzati anche a fini sanitari i presidi residenziali socio-assistenziali, soprattutto per la deospedalizzazione protetta. In tal caso la gestione è a carico dei servizi sanitari e del relativo fondo sanitario, fermo restando il supporto che viene garantito dal servizio socio-assistenziale

6. L'ospedalizzazione non è, comunque, la soluzione obbligata né per forza di cose la migliore. Le USL devono istituire il servizio di Ospedalizzazione a domicilio per tutti quei malati che possono essere curati a casa loro, aggiungendo alle prestazioni dei medici di base quelle di una équipe medico-infermieristica ospedaliera o territoriale. Ciò comporta risultati positivi per i pazienti, non sradicati dal loro ambiente; per i familiari, non costretti ad estenuanti presenze in ospedale; per il Servizio sanitario, in grado di attuare notevoli risparmi

7. Il Servizio sanitario nazionale deve dotarsi di tutti i presidi necessari per assicurare agli anziani cronici non autosufficienti, come a tutti i cittadini malati, idonei interventi preventivi, curativi e riabilitativi

8. In particolare, fermo restando il diritto dei familiari di seguire, sostenere ed aiutare i propri congiunti malati, il Servizio sanitario nazionale deve garantire il personale necessario per dare al paziente non autosufficiente tutto il supporto affinché eserciti il massimo della propria autonomia personale nelle attività basilari quali mangiare, bere, alzarsi, muoversi

9. Il volontariato, in primo luogo quello che opera a casa degli anziani cronici non autosufficienti, deve essere valorizzato nella sua funzione integrativa, e non sostitutiva, dei servizi che devono essere garantiti dal Servizio sanitario nazionale

10. In ogni caso, attualmente, nulla deve essere a carico, dal punto di vista economico, dell'anziano cronico non autosufficiente o dei suoi familiari per qualsiasi tipo di ricovero, alloggio o cura; e ciò analogamente al trattamento di cui può godere ogni altro cittadino italiano ammalato 
scompensate, con broncopatia cronica riacutizzata, con problemi cognitivi, con problemi sociali, con problemi assistenziali, con problemi economici. I pazienti cronici hanno bisogno di cure di tipo riabilitativo (per recuperare possibili livelli di autonomia), di tipo sintomatico (per alleviare le conseguenze delle patologie e della condizione di non autosufficienza) e di tipo preventivo (per evitare l'aggravamento delle patologie in essere e l'insorgere di sindromi collaterali). Sulla base di queste premesse si comprende perché soprattutto l'internista debba occuparsi dei diritti negati all'anziano. Il professionista sanitario dedicato alla gestione del paziente cronico deve avere la capacità di prendere decisioni in più ambiti e in differenti setting di cura e, frequentemente, in situazioni con ridotte evidenze scientifiche e senza linee guida di riferimento. Deve inoltre avere competenze trasversali a più ambiti di cura con una visione olistica che favorisca l'interazione tra le varie figure sanitarie impegnate nella cura del paziente garantendo una presa in carico globale dello stesso. Pertanto l'internista è uno dei principali specialisti impegnati nella gestione del paziente cronico e quindi uno dei principali professionisti che subisce la mancata politica di adeguata assistenza all'anziano, cosa che comporta la perdita del sostegno dei diritti dell'anziano. In più una mancata organizzazione socio-sanitaria per la gestione delle persone anziane si riflette anche sull'organizzazione ospedaliera: le regioni con il più basso numero di posti letto per anziani in strutture residenziali (Lazio e Sud Italia) presentano un tasso di ospedalizzazione nettamente più elevato rispetto alla media nazionale. Anche in questo caso appare evidente come un'inap- propriata sovra-offerta di carattere ospedaliero assorba impropriamente risorse destinabili a forme di assistenza all'anziano più adeguate ai suoi bisogni e meno dispendiose.

Chiudiamo da dove siamo partiti: la nostra Costituzione all'art. 32 stabilisce che La Repubblica tutela la salute come fondamentale diritto dell'individuo $e$ interesse della collettività, pertanto il Servizio Sanitario Nazionale deve garantire a tutte le persone anziane un'adeguata assistenza sanitaria, poiché anche se il paziente anziano cronico è forse inguaribile esso non è mai incurabile. Le Regioni devono programmare i posti letto degli ospedali tenendo conto delle esigenze di malati acuti, cronici riacutizzati, convalescenti e lungodegenti e le ASL devono provvedere alla tutela della salute degli anziani, fornendo le proprie prestazioni qualunque siano le cause, la fenomenologia e il decorso della malattia e comunque devono garantire prestazioni sanitarie alle persone colpite da malattie specifiche della vecchiaia e ciò indipendentemente dalla loro durata.

\section{Bibliografia}

1. WHO. Guidelines on Integrated Care for Older People (ICOPE). Available from: http://www.who.int/ageing/ publications/guidelines-icope/en/

2. Ministero della Salute. Piano nazionale della cronicità. Available from: http://www.salute.gov.it/imgs/C_17 pubblicazioni_2584_allegato.pdf

3. Commissione Europea Bruxelles. Raccomandazione; 2014. Available from: http://www.eur-lex.europa.eu/Lex UriServ/LexUriServ.do?uri=COM:2014:0022:FIN:IT:PD 


\title{
Diritti negati, abusi, maltrattamenti e neglect nell'anziano: macrofenomeno mondiale sommerso, sostanzialmente misconosciuto o trascurato
}

\author{
Pietro Calogero, ${ }^{1}$ Afro Salsi ${ }^{2}$ \\ ${ }^{1}$ Direzione UOC Geriatria, Aosp Bologna; ${ }^{2}$ Geriatria, Bologna, Italia
}

\section{Perché i vecchi}

Tutti sanno ormai del fenomeno dell'invecchiamento demografico, un processo che riguarda praticamente tutte le popolazioni del mondo le cui collettività in parte hanno già espresso questo fenomeno in parte invece dovranno ulteriormente esprimerlo. Quindi limitarsi a richiamare ancora una volta una trasformazione così squisitamente epocale e che costituisce ormai un dato di certezza assoluto da nessuno contestato, rischia apparire come operazione retorica e, paradossalmente, di sfumare fino a confondere i problemi che l'accompagnano, che invece all'opposto vanno esaminati in tutta la loro a volte drammatica concretezza. Fra i tanti ve ne è uno che ci parla dell'enorme aumento dei soggetti alle età estreme della vita - diciamo gli ultranovantenni - i quali porranno problemi da affrontare e risolvere nuovi, fino ad ora per la maggior parte inusuali, sfidanti fino ai limiti delle possibilità delle varie aree disciplinari che dovranno ognuna esprimere sensibilità e conoscenze gerontologicamente coerenti: sociologia, politica, economia, medicina, psicologia, urbanistica e altre ancora. Rifacendosi ai dati proiettivi - uno dei tanti ma certamente affidabile - dell'US Census Bureau ${ }^{1}$ elaborato quasi venti anni fa ci si dice che il numero di questi ipervecchi decuplica nell'arco di mezzo secolo ma soprattutto a partire dagli anni venti del duemila inizierà una crescita quasi logaritmica. In Italia oggi 2019 i centenari e ultracentenari sono circa 20.000 (Figura 1).

Questa fascia di popolazione e quindi a maggior ragione i soggetti quasi-coetanei, per intenderci gli ul-

Corrispondente: Afro Salsi, Geriatria, Bologna, Italia. E-mail: afro.salsi@gmail.com

Articolo pubblicato secondo la Creative Commons Attribution NonCommercial 4.0 License (CC BY-NC 4.0).

(C) Copyright P. Calogero e A. Salsi, 2019

Licensee PAGEPress, Italy

QUADERNI - Italian Journal of Medicine 2019; 7(4):4-11 tranovantenni, subirà uno sviluppo incrementale similogaritmico a partire dagli anni 2030-2040 essendo nel frattempo già triplicata nell'arco di tempo che va dal $2000 \mathrm{al} 2020$. È un fenomeno quindi già avviato e nella percezione comune. Quali gli effetti di tale invecchiamento estremo o per meglio precisare quali sono i problemi che dobbiamo affrontare senza alcuna possibilità reale di poterli eludere? Per meglio comprendere il profilo qualitativo di tale trasformazione al fine anche di individuare gli adeguamenti corretti da ricercare è utile richiamare la definizione di invecchiamento. Per invecchiamento si intende una riduzione progressiva della riserva funzionale di organi e apparati accompagnata da una altrettanto progressiva riduzione della capacità di adattamento omeostatico alle variazioni ambientali. In pratica e per semplificare allo scopo di una migliore comprensione possiamo affermare che i vecchi fanno sempre più fatica a riprendersi dai e superare i danni prodotti dagli stressori ambientali (malattie, traumi, difficoltà quotidiane e simili) ragione per cui ci appaiono come soggetti allo stesso tempo deboli e indifesi mano a mano che l'invecchiamento procede. La ricerca gerontologica ha da tempo fornito una chiave di lettura universalmente accettata dell'invecchiamento incentrata sulla definizione di fragilità riportata nel sotto paragrafo successivo con una proposta di traduzione italiana. ${ }^{2}$

\section{Definizione di fragilità}

Frailty, a fairly common biological syndrome in the elderly, is identified by decreased reserves in multiple organ systems. It may be initiated by disease, lack of activity, inadequate nutritional intake, stress, and/or the physiologic changes of aging. Frailty develops slowly in a stepwise process, with increments of decline precipitated by acute events. It is manifested as loss of skeletal muscle mass (sarcopenia), abnormal function in inflammatory and neuroendocrine systems, and poor energy regulation. In the frail elderly, there is homeostenosis, or a decreased ability in the body's physiologic response to maintain homeostasis in times of acute stress. In essence, frailty is a product of ex- 
cess demand imposed upon reduced capacity. Once the elderly become frail, there is often a rapid, progressive, and self-perpetuating downward spiral toward failure to thrive and death.

La fragilità, una sindrome biologica abbastanza comune negli anziani, è identificata da una diminuzione delle riserve in sistemi multipli di organi. Può essere provocato da malattia, mancanza di attività, insufficiente apporto nutrizionale, stress e/o dal cambiamenti fisiologici connesso all'invecchiamento. La fragilità si sviluppa lentamente in un processo graduale, con incrementi del declino precipitati da eventi acuti. Si manifesta come perdita della massa muscolare scheletrica (sarcopenia), funzione anormale nei sistemi infiammatori e neuroendocrini e scarsa regolazione energetica. Negli anziani fragili, vi è riduzione delle capacità omeostatiche ovverossia una diminuzione della capacità di risposta fisiologica del corpo a mantenere l'omeostasi in periodi di stress acuto. In sostanza, la fragilità è un il frutto di una condizione di eccesso di domanda a fronte di capacità ridotte. Una volta che gli anziani diventano fragili, si osserva spesso una spirale verso il basso rapida, progressiva e auto-perpetuantesi verso lo scompenso e la morte.

La definizione qui riportata ed il ragionamento sin qui condotto ci restituiscono chiaramente l'immagine di soggetti fragili/vulnerabili conseguentemente esposti al rischio in generale, in definitiva ogni forma di rischio e perciò anche - si potrebbe dire soprattutto quello del maltrattamento. Si tratta di individui che essenzialmente in ragione della loro fragilità clinico/biologica all'interno della specifica nicchia ecologica di rete relazionale non possono esprimere le usuali strategie difensive (fisiche, intellettive, comportamentali, sociali) per mezzo delle quali normalmente si tutelano i soggetto più giovani. È attraverso questi processi di progressiva trasformazione che invecchiando si di- venta sempre più vittime potenziali o preferite del maltrattamento. Si tratta di una condizione che può generare due reazioni fra di loro opposte: la prima, probabilmente per fortuna maggioritaria, induce alla solidarietà e alla protezione, la seconda invece caratterizzata da sentimenti meno empatici induce allo sfruttamento fino alla violenza. La cultura anglosassone nella maggioranza dei casi utilizza il termine abuse ma credo più appropriata come scelta lessicale italiana il termine maltrattamento che meglio richiama gli atti cui qui ci riferiamo. Tanto più che il Center for Diesase Control and Prevention statunitense lo definisce così: ${ }^{3}$ elder abuse (mistreatment) is an intentional act or failure to act by a caregiver or another person in a relationship involving an expectation of trust that causes or creates a risk of harm to an older adult. Mistreatment may be in the domains of physical, sexual, emotional or psychological, and financial abuse or exploitation and neglect. Quindi atti volontari compiuti con modalità attiva od omissiva nell'ambito di tutti i domini fisico, sessuale, emozionale o psicologico e finanziario.

\section{Quanti sono}

Il maltrattamento dell'anziano su scala mondiale rispetto agli studi che hanno cercato di determinarne la prevalenza nella varie popolazioni studiate presenta due riscontri costanti e pressoché generalizzati che ne segnano profondamente il profilo. Il primo è l'elevato numero di coloro che ne sono vittime dato che la forbice delle varie prevalenze osservate oscilla da un minimo di meno del $5 \%$ nel Regno Unito ${ }^{4}$ ad un massimo di quasi $30 \% .^{5}$ Una variabilità che si giustifica con la difficoltà di definire in maniera univoca e per quanto possibile standardizzata il metodo di rilevazione oltre

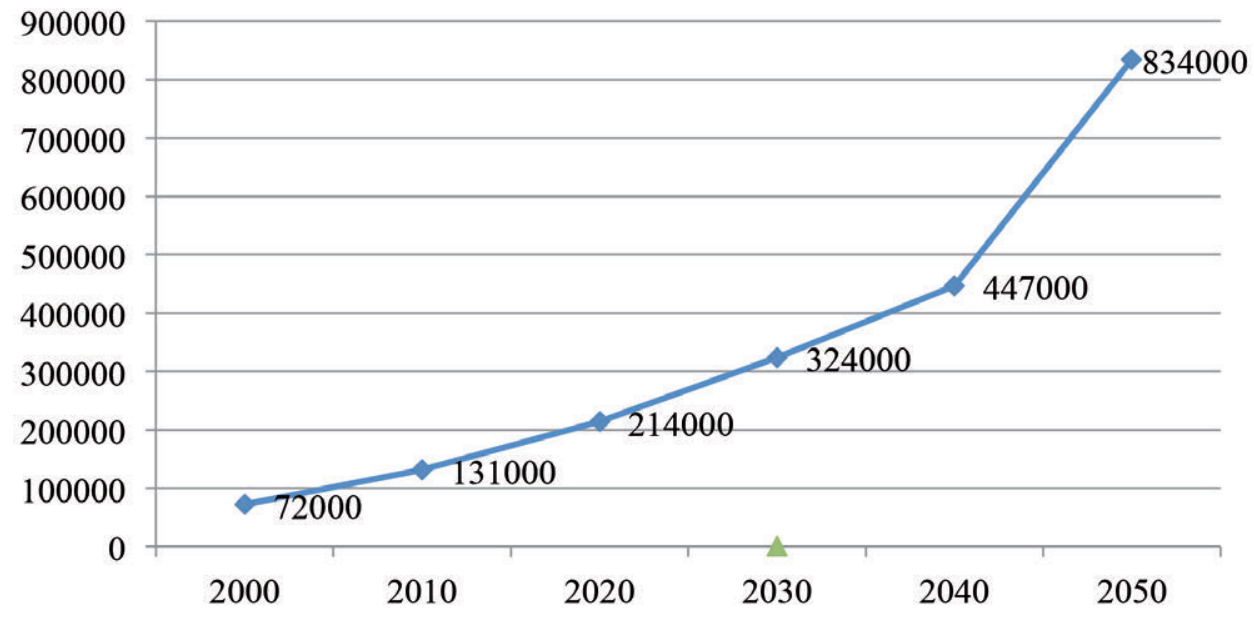

Figura 1. Centenari negli USA. 
alla soglia limite di separazione per comportamenti che per forza di cose sfumano gradualmente dalla normalità borderline all'episodio commissivo vero e proprio. Inoltre un'indagine realizzata nel Regno Unito ha potuto riscontrare che spesso mancano nel personale di assistenza la conoscenza e la sensibilità necessarie a riconoscere, registrare e segnalare $1^{\prime}$ abuso. ${ }^{6}$ Crediamo che relativamente a questo punto di non semplice quantificazione della numerosità casistica, al fine di fissare un dimensionamento di massima affidabile e orientante si possa prendere come riferimento una metanalisi recentemente pubblicata di $52^{7}$ studi da cui emerge che un anziano su sei patisce questa condizione in almeno una delle aree potenzialmente interessate. Il secondo importante tratto che accompagna il fenomeno del maltrattamento e che lo caratterizza profondamente è quello della mancata emersione e quindi della non conoscenza di una quota rilevante di episodi che ovviamente non è possibile quantificare ma della cui rilevanza tutti coloro che se ne sono occupati hanno contezza. Gli episodi di maltrattamento di vario tipo sull'anziano sono frequenti - come detto uno su sei - ma una parte rilevante di questa casistica non è conosciuta dalla collettività così che essa viene privata dell'elemento essenziale - la conoscenza - per costruire azioni contrastive. D'altronde ciò è perfettamente comprensibile se è vero, come è vero, che solitudine, isolamento, abbandono sono fattori primari di rischio. L'emersione dei casi è quindi il primo gradino di ogni percorso per il loro contenimento.

Che ci si trovi nella condizione di dover affrontare un problema dai rilevanti contorni sociali per l'impatto epidemiologico ed anche etico-morale visto che stiamo parlando di tanti soggetti iperdeboli (vecchi ma anche con tutele scarse o assenti o che gli sono addirittura ostili) lo dimostra anche il duplice intervento dell'OMS ${ }^{8,9}$ che ha prodotto due documenti sul tema rispettivamente nel 2002, quindi quasi venti anni orsono, e nel 2008. Pur non sfuggendo il limitato valore operativo di simili documenti che nella migliore delle ipotesi sono uno stimolo alla conoscenza e all'avvio di azioni concrete, in Italia scarsa o nulla eco essi hanno avuto essendosi di fatto preferito lasciare tutto nel sommerso salvo poi scandalizzarsi e inorridire quando casi a volte clamorosi venivano alla conoscenza di molti tramite i media. Ciò, come dimostrato, è solo la punta molto estrema dell'iceberg. Una delle poche iniziative assunte ${ }^{10}$ nel nostro paese è quella attuata a Udine dalla Cooperativa Solimai e denominata TAM (Telefono Anziani Maltrattati) un servizio che mette a disposizione della popolazione le funzioni di ascolto e selezione del casi con compilazione di un dossier ed eventuale attivazione di interventi. Da osservare che nel 2017 praticamente il 100\% delle chiamate era da considerarsi pertinente. Questo particolare induce a pensare che le segnalazioni scattino quando la situazione è chiaramente al di sopra di ogni dubbio di maltrattamento, il che se da un lato tranquillizza circa il funzionamento efficiente di sistema di questo tipo, dall'altro induce a credere che molti casi per varie ragioni meno eclatanti vadano persi. L'emersione del fenomeno, occorre ribadirlo, è prerequisito necessario per indurre nelle collettività una coscienza e una conoscenza del medesimo non fugace e non limitata nel tempo all'indignazione scatenata dall'episodio clamoroso. L'emersione serve infatti anche a produrre quella conoscenza dalla quale delineare una corretta strategia di approccio che non si limiti alla mera repressione/punizione del o dei perpetratori - che si sono dimostrate di scarsa efficacia - ma che al contrario sappia avvalersi anche di interventi probabilmente più appropriati come il supporto al disagio e la possibilità di stemperare le condizioni di isolamento e solitudine. Emersione, repressione, aiuto e lotta all'isolamento sociale sono le quattro linee operative lungo le quali dovrebbe svilupparsi un programma sociale di lotta al maltrattamento dell'anziano da parte di una collettività. Credo che possa riassumersi con questa enunciazione la sintesi dei tentativi fin qui condotti nelle varie realtà in tutto il mondo.

Per quanto attiene all'opera di emersione, la costituzione di punti raccolta delle segnalazioni di facile fruizione da parte di tutti i cittadini e perciò facilmente accessibili e con percorsi massimamente deproceduralizzati e deformalizzati in grado di assicurare a chi segnala ogni garanzia possibile circa le sempre temute ritorsioni rappresenta la migliore delle opzioni possibili. L'opzione dei punti di raccolta delle segnalazioni prodotte dalla varietà di soggetti a qualunque titolo testimoni potenziali è preferibile rispetto alla ricerca attiva, magari supportata da schede-intervista ad hoc create, delle situazioni in essere o a rischio (cosiddetto case finding) che invece appare più adatta a studiare il maltrattamento in una specifica popolazione nei suoi vari aspetti quali ad esempio la dimensione o i fattori di rischio. Sono disponibili questionari strutturati validati con punteggi utili allo scopo di capire se in un determinato contesto sussistano condizioni di maltrattamento. ${ }^{11}$

\section{Quali i fattori di rischio}

Il primo e più importante fattore di rischio naturalmente ed intuitivamente è la vecchiaia in quanto tale soprattutto se, come detto, molto avanzata e accompagnata da un grado elevato di fragilità/vulnerabilità. Quindi con l'incremento del grado e del numero di malattie e delle connesse disabilità - si ricordi che l'età anagrafica è proporzionale al numero delle comorbidità presenti il quale numero peraltro resta comunque estremamente variabile da individuo a individuo - si assiste ad un parallelo incremento del rischio. ${ }^{12} \mathrm{La}$ comparsa di una o più malattie nella vicenda indivi- 
duale di una persona produce molteplici modificazioni del profilo fenomenologico del sè in tutte le aree relazionali - somatica e psicologica ma anche ovviamente quella delle capacità prestazionali - tramite le quali, in definitiva, si interagisce e ci si rapporta col mondo esterno che può a volte finire col percepirci in modo spesso negativo, progressivamente deteriorato rispetto ai vissuti precedenti. Ciò può avvenire esemplarmente nel caso di sindromi dementigene laddove il disfacimento progressivo e totale della personalità rischia di minare alla base una preesistente affettività, un fenomeno che assommandosi alla fatica e allo stress dell'accudienza bene spiega, certo non giustifica, molti dei casi di maltrattamento. In questo senso colpirono la lucidità e la profondità della redazione del New York Times quando anni addietro riportò la notizia di un anziano, affetto da grave demenze, che venne abbandonato solo su di una carrozzina a Central Park: when love exhausts fu il titolo con cui venne data notizia dell'episodio. Ritengo che si debba avere piena e solida contezza di questo ordine di elementi se si vogliono predisporre azioni di ostacolo efficaci che vadano oltre la scorciatoia della repressione che pure ha un ruolo innegabile da non sottovalutare, forse inferiore alle aspettative.

Contrariamente rispetto a quanto si sarebbe indotti a pensare la maggioranza degli episodi, che approssimativamente e coi limiti di stima già detti si attesta sul $60 \%$, ha luogo in ambito domestico. Ciò stupisce solo fino ad un certo punto perché è ovviamente in quel tipo di contesto che lo stress assistenziale e quindi emotivo dei caregivers può raggiungere il massimo ed è nel medesimo contesto che minime all'opposto sono le forme di controllo sociale. Un'indagine realizzata in sette paesi europei ${ }^{13}$ intervistando 4464 anziani di età compresa fra 60 e 84 anni non dementi e non istituzionalizzati conclude confermando l'ipotesi che status socio economico e supporto sociale di cui l'anziano può fruire, in definitiva due variabili fortemente interconnesse l'una con l'altra, siano un importante fattore di protezione o all'opposto, se carenti, di rischio. In questi casi la condizione di debolezza economica e sociale produce un effetto di via finale comune costituita dall'isolamento che priva la persona anziana di buona parte delle difese potenziali intrinseche ed estrinseche. A prima vista curioso ma non del tutto inaspettato è invece il riscontro di una relazione, documentata, fra il turn-over del personale e la possibilità di subire maltrattamenti per gli anziani ospiti di strutture. ${ }^{14}$ Collateralmente appare allarmante quanto emerge dallo stesso studio condotto in 300 strutture long-term di Israele che ha rivlevato come oltre la metà degli ospiti avesse subito una qualche forma di violenza nei 12 mesi precedenti. Certamente l'alto turn-over del personale è di per sè indicatore di una quasi certa non ottimale qualità dell'assistenza e per- ciò di un terreno fertile per il determinarsi di eventi di abuso, ma è anche vero che proprio questo tipo di turn-over accelerato ostacola il sedimentarsi di quelle forme di professionalità e sensibilità che possono costituire un argine ai maltrattamenti.

In tema di analisi dei principali fattori di rischio o all'opposto di protezione è senz'altro di un certo interesse ricordare come gli anziani vittime di abuso possano adottare strategie e tecniche per quanto possibile di difesa individuale e queste sembrano essere di una qualche efficacia in particolare quando sono imperniate sul mantenimento o addirittura sul rafforzamento dell'immagine del sé, un obiettivo questo decisamente soggetto-dipendente ma non per questo impossibile da perseguire. ${ }^{15}$ Ovviamente sono protettivi anche quei comportamenti cautelativi che cerchino di evitare di esporre il soggetto a situazioni propizianti l'evento abuso, quali ad esempio l'assenza di una o più persone di fiducia con cui comunicare o la rarefazione dei rapporti sociali o, peggio ancora, la decontestualizzazione totale del rapporto fra l'anziano ed il suo care-giver primario quando la relazione con chi lo assiste rimane l'unica possibile. Nella Tabella 1 che segue sono riportati i più significativi fattori di rischio.

\section{Le azioni contrastive}

Chi maltratta un anziano nelle varie forme attraverso le quali questa spregevole azione può esprimersi, seconda la legge italiana rischia l'imputazione in ordine ad alcuni reati. Essi sono: circonvenzione d'incapace, truffa, lesioni personali, abuso di mezzi di contenzione o disciplina, abbandono di incapace, violazione e omessa prestazione dei mezzi di sussistenza e probabilmente altri ancora. Quindi gli strumenti giuridici da applicare certo non mancano ma, ancora una volta, se non vi è un'emersione degli episodi che vada oltre quei singoli casi limite riportati dagli organi di informazione, anche la repressione e l'erogazione di una pena - forma di contrasto di per sé dal valore limitato - di fatto si trasforma in un'arma sostanzialmente spuntata. Le azioni di contrasto, in particolare quelle di dimostrata efficacia, sono come immaginabile di non semplice costruzione ed in particolare nel nostro paese assai scarse tanto che l'Italia non è neppure presente nel report del $20011 \mathrm{col}$ quale l'OMS ha cercato di delineare una strategia di prevenzione nei confronti dei maltrattamenti agli anziani. ${ }^{16}$

Negli USA sono progressivamente sorti punti di riferimento a variabile tipologia funzionale per coloro che necessitano di supporto in ordine a situazioni di abuso come l'Elder Abuse Prevention Unit in Virginia uno dei primi e attivo dal 1997, istituito a seguito dei preoccupanti dati di prevalenza emersi da un'indagine di tre anni prima. Essenzialmente sono centri di raccolta di segnalazioni, elaborazione delle medesime ed 
attivazione di quanto localmente posibile in termini di prevenzione. In un sito web $^{17}$ dedicato alla popolazione anziana si contano censiti 75 programmi a vario titolo connessi al tema della prevenzione dell'abuso.

Delle difficoltà di reperire dati a supporto della dimostrazione scientificamente valida, in altri termini basata sul criterio principale dell'evidenza sperimentalmente acquisita, ci offrono testimonianza due contributi realizzati col metodo della metanalisi. La prima del 2009 conclude che negli studi presi in considerazione vi sono importanti limiti metodologici tali per cui non è possibile trarre elementi di una qualche utilità circa l'efficacia degli interventi realizzati. ${ }^{18} \mathrm{La}$ seconda più recente in quanto del 2017 e avente per oggetto la condizione di domiciliarità, forse la più difficile da affrontare, conclude da un lato confermando quanto emerso dalla precedente e dall'altro lamentando la scarsità dei risconrti presenti in letteratura. ${ }^{19}$ Stando invece a quanto osservato grazie ad un intervento concepito a scopo preventivo ma anche di gestione dei casi rilevati, su una popolazione di 558 anziani ospiti di strutture in tre municipalità dello stato di Israele e basato principalmente sul counseling si possono ottenere un miglioramenti nel $66 \%$ dei casi e l'azzeramento nel $20 \%$. Interessante notare che secondo gli autori dello studio i migliori risultati si ottennero grazie alla protezione legale. Un risultato un po' in controtendenza ma che forse si spiega col contesto istituzionale ove, di norma, il controllo sociale nelle sue varie forme è maggiore e quindi anche il potenziale di deterrenza da esso derivato. ${ }^{20}$ Anche stando alle indicazioni fornite dal documento della Regione Europea dell'OMS già citato ${ }^{16}$ per la maggior parte delle tipologie di intervento che si possono ipotizzare e perciò in teoria attuare non vi è certezza, come già detto, circa i risultati ottenibili. Purtuttavia la preparazione e la sensibilizzazione professionale del personale, i programmi scolastici che migliorino la relazione intergenerazionale e con essa l'attitudine dei giovani verso gli anziani, la rieducazione psicologicocomportamentale di chi maltratta, forme di tutela legale sembrano offrire qualche risultato degno di attenzione. Vengono richiamate, fra le altre, a titolo esemplificativo un'esperienza tedesca di addestramento del personale a riconoscere e gestire i casi di maltrattamento al domicilio, una irlandese di workshops sul tema della violenza all'anziano per assistenti sociali e per studenti di scuole di formazione sanitaria, una spagnola di un sito web dedicato al tema con una sorta di decalogo per la prevenzione.

Probabilmente di non irrilevante interesse, sia ai fini di una comprensione non superficiale degli abusi sia per opporvisi coi giusti strumenti, sono i risultati ottenuti nel Regno Unito grazie a programmi di ri-educazione e gestione dell'aggressività, diretti a persone che se ne erano rese responsabili o comunque ad alto rischio, tutti care-givers informali che agivano al domicilio dove, occorre ribadirlo, maggiore è la possibilità che tali eventi abbiano luogo. ${ }^{21}$ Il programma, che era strutturato in sessioni individuali della durata di novanta minuti incentrate sull'addestramento e sul controllo dell'aggressività (parte I e II) produsse una significativa riduzione delle componenti determinanti maggiori nella relazione conflittuale e perciò degli abusi. Si può ritenere, a mio avviso con qualche fondamento, che questo sia il cuore del problema più ge-

Tabella 1. Fattori di rischio.

\begin{tabular}{|c|c|}
\hline Vittima & $\begin{array}{l}\text { Genere femminile } \\
\text { Età superiore } 75 \text { aa } \\
\text { Alto livello di disabilità fisica e intellettuale }\end{array}$ \\
\hline Perpetratore & $\begin{array}{l}\text { Genere maschile } \\
\text { Disturbi mentali } \\
\text { Abuso di sostanze } \\
\text { Ostilità } \\
\text { Difficoltà economiche } \\
\text { Stress e burn-out }\end{array}$ \\
\hline Relazionali & $\begin{array}{l}\text { Dipendenza economica del perpetratore dalla vittima } \\
\text { Dipendenza emotiva o logistica del perpetratore dalla vittima } \\
\text { Storia di difficoltà relazionali } \\
\text { Ambiente violento } \\
\text { Sistemazione logistica carente } \\
\text { Relazione di figlio o partner }\end{array}$ \\
\hline Comunità & $\begin{array}{l}\text { Isolamento sociale } \\
\text { Carenza di supporti }\end{array}$ \\
\hline Sociali & $\begin{array}{l}\text { Ageismo } \\
\text { Razzismo o sessismo } \\
\text { Cultura della violenza } \\
\text { Problemi sociali o economici }\end{array}$ \\
\hline
\end{tabular}


nerale e che quindi le azioni da intraprendere più efficaci siano principalmente quelle indicate, vale e dire quelle che operano sull'asse della care: competenza di chi accudisce, supporto assistenziale, gestione dell'aggressività, livelli di stress. Lo studio citato purtroppo mancava dei casi controllo e quindi non può essere considerato fra quelli di buona qualità, nonostante ciò ritengo che le indicazioni che se ne possono ricavare siano quelle giuste. Del resto secondo la già citata fonte bibliografica dell'OMS ${ }^{16}$ sarebbero proprio in generale i programmi di supporto ai care-givers quelli che danno i risultati maggiori. Più recentemente ${ }^{22}$ a conferma dell'ipotesi che proprio nella diade assistito/perpetratore e nel profilo particolare che tale rapporto assume si collochino le radici della maggior parte dei fenomeni di maltrattamento e che da questo si dovrebbe partire per azioni ostative sono stati resi disponibili i risultati di uno studio iraniano. L'interesse particolare dello studio sta anche nella dimostrazione indiretta della dimensione transculturale del fenomeno, visto che parliamo di una collettività musulmana, e quindi possiamo assumere che il rischio di violenza sugli anziani fragili e vulnerabili sia una condizione universalmente presente. Ovviamente è soprattutto sui risultati ottenuti che esso richiama la nostra attenzione. Col metodo caso-controllo un totale di 464 perpetratori di abusi (232 trattati e 232 controlli) della regione di Teheran sono stati sottoposti a sessioni di 45-60 minuti per un ciclo complessivo di 20 sedute nell'arco di 6 mesi. Al termine del ciclo si potè osservare un significativo positivo effetto sui trattati rispetto ai controlli.

Le principali considerazioni fin qui svolte trovano una sintesi efficace ed una conferma nel documento elaborato dalla Commissione per le Politiche della Famiglia dello Stato di Komihana e Whanau in Nuova Zelanda grazie ad un'analisi realizzata mediante interviste strutturate face to face ai principali soggetti a qualsiasi titolo presenti sulla scena, a interviste telefoniche e a focus groups. ${ }^{23} \mathrm{Il}$ materiale così raccolto è stato elaborato alla luce della letteratura scientifica e proposto alla collettività locale e internazionale per mezzo di un documento che si raccomanda alla lettura per la sua coerenza e condivisibilità di contenuti, metodologia seguita e completezza delle informazioni ivi riportate quanto a epidemiologia, fattori di rischio e protezione, azioni preventive e correttive suggerite. Relativamente a queste ultime, vale a dire le azioni correttive, vi è un contributo che merita di essere pure ricordato. Si tratta della proposta metodologica ${ }^{24}$ di un gruppo californiano formulata dopo l'analisi di oltre mille casi che, richiamandosi anche ai principi della Valutazione Geriatrica Multidimensionale, raccomanda l'adozione dell'Abuse Intervention Model (AIM) per valutare, processandolo, ogni caso. Esso richiede la scomposizione del contesto in tre domini principali che sono: Vulnerable Older Adult, Trusted
Other (possiamo dire la persona cui l'anziano si affida) e Context. Ciascuno di questo domini ha una serie di variabili che tutte devono essere analizzate e prese in considerazione per possibili azioni correttive. Come si vede molte sono le analogie con la citata Valutazione Geriatrica Multidimensionale.

Si è detto che il primo passo di un azione virtuosa di contenimento è probabilmente l'emersione della casistica. Per ottenere ciò occorre creare stimoli affinchè ciò avvenga e istituire perciò un punto di raccolta, che definiremo Centro Unico Multitask di Raccolta delle Segnalazioni (CUMRS) in grado anche di analizzare le segnalazioni acquisite individuando quelle meritevoli di un seguito. Un punto, unico in ogni territorio, che ha necessità di essere socialmente visibile e riconoscibile oltrechè facilmente accessibile grazie all'assenza di ogni forma complessa di fruizione. Deve inoltre essere dotato di un set minimo di competenze comprensivo di conoscenze di ordine psicologico, giuridico, medico geriatrico, sociale $o$ altre che si ritenessero utili. In questo modo si ottiene la realizzazione di una sorta di registro locale dei maltrattamenti, che di per sé ha una capacità intrinseca di sensibilizzazione e catalizzazione di azioni proattive utili a combattere i maltrattamenti, e si fornisce una risposta a quei casi ritenuti meritevoli per importanza e gravità dell'episodio. L'epidemiologia del fenomeno mondiale dell'elder abuse e i dati di prevalenza che vi si collegano dimostrano che vi è necessità di iniziative appropriate e che tali iniziative, stando alle indicazioni emerse dal complesso delle pubblicazioni scientifiche sul tema, debbano imperniarsi sul modello operativo riportato nello schema in Figura 2.

Secondo tale schema al Centro Unico o come altro lo si voglia definire compete, oltre alla raccolta della casistica anche in una logica di screening per predisporre interventi nelle situazioni più eclatanti, elaborare suggerimenti per la programmazione dei servizi, l'onere di iniziative quali campagne di sensibilizzazione per promuovere atteggiamenti postivi nei riguardi della popolazione anziana fragile, la formazione specifica per cogliere e prevenire i maltrattamenti per gli staff con compiti di assistenza nelle aree più esposte al rischio, corsi per care-givers che creino le condizioni utili allo svolgimento del compito e alla gestione dell'aggressività insieme allo stress. In definitiva si tratta di dotarsi di uno dei tanti di strumenti necessari per adeguare le nostre collettività al fenomeno dell'iperinvecchiamento.

\section{Punti chiave}

- L'iperinvecchiamento in particolare estremo è la causa principale dei maltrattamenti agli anziani.

- La maggior parte dei maltrattamenti sono misconosciuti.

- L'emersione del fenomeno è prerequisito irrinunciabile per opporvisi. 


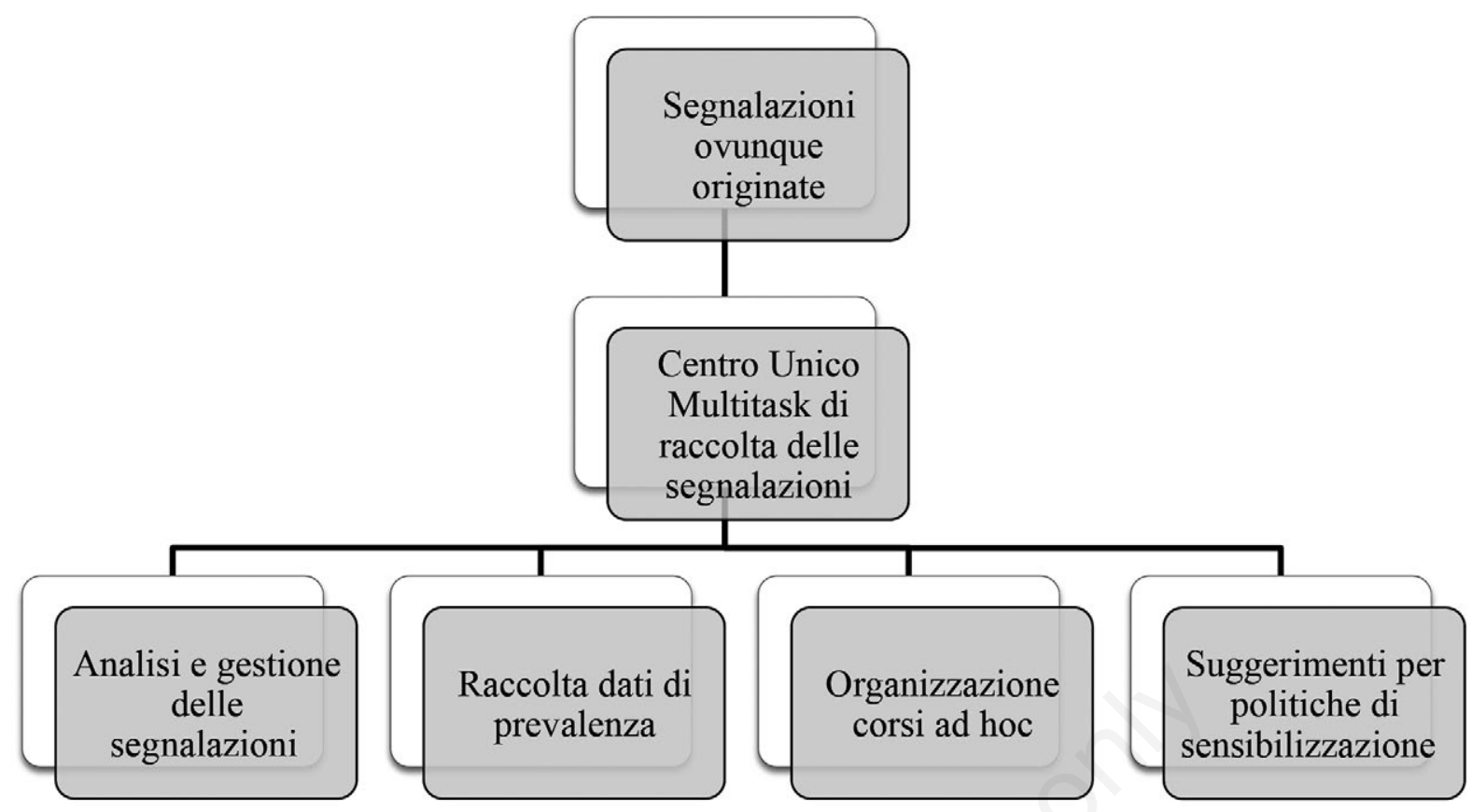

Figura 2. Modello operativo da attuare in caso di elder abuse.

- Un'efficace prevenzione si basa sulla creazione di centri di segnalazione multitasking.

\section{Conclusioni}

I maltrattamenti agli anziani e in particolare se iper-longevi sono eventi di accertata elevata frequenza su scala mondiale e, soprattutto e purtroppo, ampiamente misconosciuti e in Italia non affrontati nonostante appaiano comportamenti spregevoli sul piano etico e morale. L'urgenza di azioni contrastive richiede iniziative non dilazionabili ancorchè non evidence based. Purtuttavia un'attenta analisi della letteratura scientifica disponibile consente di delineare ipotesi di interventi di una qualche efficacia anche se ovviamente non in grado di azzerare gli episodi. L'ipotesi della creazione di un Centro Unico Multitask a configurazione e funzionamento variabili a seconda del contesto rappresenta lo schema concettuale per agire.

\section{Bibliografia}

1. US Census Bureau. Midrange estimate for centenarians in the United State; 1999.

2. Ahmed N, Mandel R, Fain MJ. Frailty an Emerging Geriatric Syndrome. Am J Med 2007;120:748-53.

3. Hall JE, Karch DL, Crosby AE. Elder Abuse Surveillance: Uniform Definitions and Recommended Core Data Elements for Use in Elder Abuse Surveillance, Version 1.0. Atlanta, GA: National Center for Injury Pre- vention and Control, Centers for Disease Control and Prevention; 2016.

4. Biggs S, Manthorpe J, Tinker A, et al. Mistreatment of older people in the United Kingdom: findings from the first national prevalence study. J Elder Abuse Negl 2009;21:1-14.

5. Garre-Olmo J, Planas-Pujol X, Lopez-Pousa S, et al. Prevalence and risk factors of suspected elder abuse subtypes in people aged 75 and older. J Am Geriatr Soc 2009;57:815-922.

6. Richardson B, Kitchen G, Livingston G. The effect of education on knowledge and management of elder abuse: a randomized controlled trial. Age Ageing 2002;31:335.

7. Yon Y, Mikton CR, Gassoumis ZD, Wilber KH. Elder abuse prevalence in community settings: a systematic review and meta-analysis. Lancet Glob Health 2017; 5:e147-56.

8. WHO. The Toronto declaration on the global prevention of elder abuse; 2002.

9. WHO. A global response to elder abuse and neglect Building primary health care capacity; 2008.

10. Cooperativa Solimai. Telefono anziani maltrattati. Disponibile a: http://www.solimai.it/solimai/index.jsp? codicePagina=TAM

11. Gonzalez, Jennifer M. Reingle Pilot testing the detection of elder abuse through emergency care technicians (DETECT) screening tool: results from the DETECT pilot project Journal of Elder Abuse \& Neglect 2019 [Epub ahead of print].

12. Dong X, Simon M, Evans D. A population-based study of physical function and risk for elder abuse reported to social service agency: findings from the Chicago health and aging project. J Appl Gerontol 2014;33:808-30. 
13. Melchiorre MG, Chiatti C, Lamura G, et al. Social support, socio-economic status, health and abuse among older people in seven European countries. PLoS One 2013;8(1).

14. Ben Natan M, Ariela L. Study of factors that affect abuse of older people in nursing homes. Nurs Manag (Harrow) 2010;17:20-4

15. Sandmoe A, Hauge S. When the struggle against dejection becomes a part of everyday life: a qualitative study of coping strategies in older abused people. J Multidiscip Healthc 2014;7:283-91.

16. World Health Organization. Regional Office for Europe. European report on preventing elder maltreatment. Copenhagen: WHO Regional Office for Europe; 2011.

17. Worldwide programs providing a better way to deal with aging. Available from: http://www.programsforelderly.com

18. Ploeg J, Fear J, Hutchison B, et al. A systematic review of interventions for elder abuse. J Elder Abuse Negl 2009;21:187-210.

19. Fearing G, Sheppard CL, McDonald L, et al. A systematic review on community-based interventions for elder abuse and neglect. J Elder Abuse Negl 2017;29: 102-33.

20. Alon S, Berg-Warman A. Treatment and prevention of elder abuse and neglect: where knowledge and practice meet-a model for intervention to prevent and treat elder abuse in Israel. J Elder Abuse Negl 2014;26:150-71.

21. Campbell Reay AM, Browne KD. The Effectiveness of Psychological Interventions With Individuals Who Physically Abuse or Neglect Their Elderly Dependents. J Interpers Violence 2002;17:416-31.

22. Estebsari F, Dastoorpoor M, Mostafaei D, et al. Design and implementation of an empowerment model to prevent elder abuse: a randomized controlled trial. Clinical Interventions in Aging 2018;13:669-79.

23. Families Commission. Kominhana a Whanau Elder abuse and neglect: exploration of risk and protective factors; 2008.

24. Mosqueda L, Burnight K, Gironda MW, et al. The Abuse Intervention Model: A Pragmatic Approach to Intervention for Elder Mistreatment. J Am Geriatr Soc 2016; 64:1879-83. 


\title{
Il diritto al coordinamento delle cure e alla continuità assistenziale nella gestione delle malattie croniche: strumenti disponibili oggi e quali potenzialmente fruibili in futuro?
}

\author{
Francesco Orlandini \\ Direzione Sanitaria, ASL4 Regione Liguria, Chiavari (GE), Italia
}

\section{Introduzione}

Il Piano Nazionale Cronicità, Accordo tra lo Stato, le Regioni e le Province Autonome di Trento e di Bolzano del 15 settembre 2016, ${ }^{1}$ emesso dalla Direzione Generale della Programmazione Sanitaria del Ministero della Salute, prendendo le mosse dalla definizione OMS di cronicità comem problemi di salute che richiedono un trattamento continuo durante un periodo di tempo da anni a decadi e dai relativi Piani d'azione WHO 2008-2013 e 2013-2020 che sottolineano l'importanza dell'intervento di Governi e Amministrazioni per assicurare la prevenzione ed il controllo delle malattie croniche, riconoscendo che $i l$ mondo della cronicità è un'area in progressiva crescita che comporta un notevole impegno di risorse, richiedendo continuità di assistenza per periodi di lunga durata e una forte integrazione dei servizi sanitari con quelli sociali pone le basi per il diritto al coordinamento delle cure e alla continuità assistenziale dei malati cronici. A ciò si aggiunge la presenza di interventi sanitari e sociosanitari integrati nei livelli essenziali di assistenza a partire dal DCPM del novembre $2001^{2}$ e l'introduzione della continuità assistenziale nel nuovo decreto $2016 .{ }^{3}$ Non ultima la legge 24/2017 che estende il diritto alla salute, includendo la sicurezza e l'appropriatezza delle cure. Altri obiettivi che richiedono per il conseguimento coordinamento e continuità delle cure.

Il paziente cronico è più spesso un pluri-cronico, cioè una persona, solitamente anziana, affetta da più pa-

Corrispondente: Francesco Orlandini, Direzione Sanitaria, ASL 4 Regione Liguria, via Ghio 9, Chiavari (GE), Italia. E-mail: orlandinif@libero.it

Articolo pubblicato secondo la Creative Commons Attribution NonCommercial 4.0 License (CC BY-NC 4.0).

CCopyright F. Orlandini, 2019

Licensee PAGEPress, Italy

QUADERNI - Italian Journal of Medicine 2019; 7(4):12-20 tologie croniche incidenti contemporaneamente (comorbidità o multimorbidità), le cui esigenze assistenziali sono determinate non solo da fattori clinici, ma anche sociali, culturali, ambientali, ecc. La presenza di pluri-patologie richiede l'intervento di diverse figure professionali ma c'è il rischio che i singoli professionisti intervengano in modo frammentario, focalizzando l'intervento piu sul trattamento della malattia che sulla gestione del malato nella sua interezza, dando talvolta origine a soluzioni contrastanti, con possibili duplicazioni diagnostiche e terapeutiche che contribuiscono all'aumento della spesa sanitaria e rendono difficoltosa la partecipazione del paziente al processo di cura.

\section{Modelli internazionali di coordinamento delle cure e continuità assistenziali}

Gli Integrated Care Models, descritti in letturatura, possono essere distinti in base al livello a cui avviene l'integrazione. Si va dai modelli progettati per integrare l'assistenza per gli individui con condizioni croniche a modelli specifici di malattia e modelli integrati basati sulla popolazione. ${ }^{4-6}$

\section{Integrazione a livello individuale}

Questo gruppo di modelli di assistenza integrata si occupa del coordinamento individuale ${ }^{5}$ delle cure per $\mathrm{i}$ pazienti ad alto rischio e/o con più condizioni e i loro accompagnatori. I singoli modelli di assistenza integrata mirano a facilitare l'erogazione adeguata dei servizi di assistenza sanitaria e a superare la frammentazione tra i diversi professionisti. Il coordinamento delle cure per questi pazienti va oltre un episodio di cura, dove è necessario il coordinamento, ma abbraccia anche il concetto di integrazione lungo tutto il corso della vita. Fra questi ricordiamo il Case Management che mira ad assicurare il coordinamento dell'assistenza di un paziente attraverso l'assegnazione di un responsabile del caso. Il ruolo di case manager può essere designato a case manager certificati con o senza esperienza medica, nonché a infermiere di cure primarie avanzate o altri pro- 
fessionisti della salute. Le componenti cruciali sono: i) selezione degli individui per i quali la gestione dei casi è più appropriata, ad esempio pazienti con multimorbilità, pazienti frequentemente ammessi in ospedale o pazienti che necessitano di coordinamento dell'assistenza sanitaria e sociale; ii) valutazione e pianificazione della cura individuale; iii) monitoraggio regolare dei pazienti; e iv) adeguamento dei piani di assistenza, se necessario. I compiti principali di un case manager sono quindi di valutare le esigenze del paziente e del caregiver, sviluppare un piano di cura su misura, organizzare e regolare di conseguenza i processi di cura, monitorare la qualità dell'assistenza e mantenere il contatto con il paziente. Le evidenze dimostrano che tale modello riduce il numero di ricoveri ospedalieri e migliora la soddisfazione dei pazienti, mentre le evidenze di costi-efficacia rimangono controverso, soprattutto se non indirizzato a gruppi di pazienti ben selezionati.

Il piano di assistenza individuale (PAI) è un altro approccio di integrazione dell'assistenza per i pazienti con condizioni di multimorbilità e a lungo termine. L'obiettivo è fornire un'assistenza più personalizzata e mirata, creando piani di assistenza condivisi che mappino i processi di cura, articolino chiaramente il ruolo di ciascun fornitore e paziente nel processo di cura e conservino informazioni retrospettive e prospettiche sull'assistenza per un particolare paziente. I piani di assistenza sono un punto di riferimento per qualsiasi operatore coinvolto nell'assistenza di un individuo. I coordinatori dell'assistenza valutano i bisogni di un paziente, sviluppano piani di assistenza e negoziano e coordinano l'erogazione di cure multidisciplinari. Il successo dei piani di assistenza che si estendono all'interfaccia di settori sanitari e sociali si basano sulle capacità di tali coordinatori. Analogamente al Case Management, i PAI sono costosi e laboriosi. Pertanto, richiedono criteri di ammissibilità chiari e un'attenta selezione dei beneficiari. Nello sviluppo di piani di assistenza è importante che il paziente e/o il caregiver siano partecipi e comprendano il piano. Inoltre, i piani di assistenza dovrebbero essere di natura dinamica, adeguati attraverso valutazioni periodiche e feedback dei pazienti. L'uso della information technology (IT) è fondamentale per facilitare lo sviluppo di piani di assistenza condivisi a cui i fornitori possono accedere attraverso contesti, pazienti e assistenti.

\section{Patient-centred medical home}

Il modello patient-centred medical home (PCMH) è una pratica di gruppo diretta dal medico che può fornire assistenza che è accessibile, continua, completa $\mathrm{e}$ coordinata e erogata nel contesto della famiglia e della comunità. Il modello PCMH adotta un approccio olistico alla gestione dei pazienti con malattie croniche, con multimorbidità offrendo un modello individuale alternativo di assistenza primaria in cui i pazienti sono assegnati a particolari gruppi di medici. Pertanto, PCMH non dovrebbe essere considerato come un contesto in cui viene fornita assistenza, ma piuttosto come un modello completo di organizzazione che fornisce le funzioni fondamentali dell'assistenza primaria. Il modello PCMH sostiene che tutti i tipi di assistenza, ad es. assistenza sub-specialistica, ospedali, agenzie sanitarie domestiche, case di cura e parti interessate, ad es. comunità, famiglia, servizi pubblici e privati basati sulla comunità, dovrebbero essere riuniti attraverso l'integrazione reale o virtuale. Una caratteristica distintiva del modello PCMH è che offre un'assistenza più personalizzata assegnando i pazienti ai medici di base, assicurando che i pazienti sappiano chi è responsabile della loro salute e che i professionisti coinvolti conoscano i pazienti di cui sono responsabili. PCMH è visto come l'apripista che cura - al contrario di gestire o limitare l'accesso - attraverso il coordinamento attivo delle persone verso le cure più appropriate. Ciò significa che gran parte dell'assistenza viene fornita da team multidisciplinari nell'assistenza primaria, ma nel caso in cui sia necessaria l'assistenza specialistica, i team di assistenza primaria acquistano questi servizi per conto del paziente. I registri dei pazienti svolgono un ruolo fondamentale nel successo dei modelli PCMH insieme alle tecnologie informatiche e agli scambi di informazioni sanitarie in modo che i pazienti ricevano servizi indicati quando e dove ne hanno bisogno in modo culturalmente e linguisticamente appropriato. La valutazione del modello PCMH mostra che ciò comporta una riduzione del $20 \%$ dei ricoveri ospedalieri e una riduzione del $12 \%$ dei tassi di riammissione tra i suoi beneficiari. Le critiche ammoniscono che in sistemi altamente frammentati, l'integrazione tra i fornitori potrebbe non avvenire a meno che i meccanismi di rimborso non incentivino specificamente l'integrazione.

\section{Budget per la salute personale}

Partendo dal presupposto che il coordinamento delle cure può essere meglio eseguito dai pazienti stessi, i budget sanitari personali sono un modello di assistenza integrata che offre ai pazienti una maggiore autonomia nella loro cura. Negli ultimi due decenni, tali modelli sono stati sperimentati negli Stati Uniti e nel Regno Unito nel settore dei servizi a lungo termine a casa e in comunità e ora sono attuati in Austria, Germania, Paesi Bassi e Norvegia. Il modello suggerisce che agendo come detentori del budget, i pazienti possono coordinare meglio la loro assistenza in base alle loro esigenze acquistando servizi dai vari professionisti. I pagamenti in contanti o i budget virtuali possono essere utilizzati non solo per i servizi di acquisto, ma anche per supportare i familiari come badanti. Le valutazioni di tale modello mostrano livelli più elevati di soddisfazione del paziente e tassi più bassi di bisogni insoddisfatti rispetto ai modelli convenzionali di 
assistenza, specialmente tra i pazienti più giovani con disabilità. Le valutazioni hanno anche dimostrato una maggiore efficienza nell'assistenza erogata, principalmente a causa di una migliore continuità ed evitamento delle duplicazioni. Tuttavia, gli studi dimostrano che gran parte degli oneri amministrativi e del rischio ricade sui pazienti e sui loro caregiver, e quindi richiede la capacità del paziente di gestirli. La qualità e il volume dei servizi acquistati dipendono pesantemente dalla capacità dei pazienti o dei caregiver di valutarla adeguatamente, il che è difficile data l'asimmetria informativa nella salute.

\section{Integrazione a livello di gruppi o malattie}

\section{Chronic care model}

Il chronic care model (CCM) è uno dei modelli di cura integrati più noti e ampiamente applicati. Il CCM è stato sviluppato nel 1998 da un gruppo di ricercatori del MacColl Institute for Healthcare Innovation in USA. Tale modello suggerisce di passare dall'assistenza acuta, episodica e reattiva all'assistenza che abbraccia approcci longitudinali, preventivi, basati sulla comunità, e integrati. Sviluppato come risultato di un'ampia revisione sistematica della letteratura, la progettazione del CCM riunisce fattori e componenti basati su evidenze ad impatto positivo sugli esiti dei pazienti, sulla qualità dell'assistenza e sui risparmi sui costi. ${ }^{5} \mathrm{Il} \mathrm{CCM}$ è costituito da sei domini principali: comunità, sistema sanitario, supporto all'autogestione, progettazione del sistema di erograzione, supporto decisionale e sistemi informativi. Ciascuno dei domini può essere ulteriormente decompresso in strategie essenziali per conseguire l'integrazione. Ad esempio, all'interno del dominio della comunità, una strategia chiave consiste nel mobilitare risorse della comunità, come partnership con organizzazioni di comunità e creazione di programmi comunitari. Il modello è stato successivamente rivisto nel 2003. In tale occasioni componenti come la competenza culturale, la sicurezza del paziente, il coordinamento dell'assistenza, le politiche comunitarie e la gestione dei casi sono state aggiunte allo schema iniziale. Le chiavi del successo del CCM sono state la comunicazione produttiva, bidirezionale all'interno del team multidisciplinare e la fornitura di supporto continuo ai pazienti per l'auto-cura. Le revisioni condotte in relazione all'efficacia sui costi del CCM hanno dimostrato che il rendimento degli investimenti iniziali richiede tempo Evoluzioni del CCM sono l'assistenza innovativa dell'OMS per il quadro delle condizioni croniche e il modello espanso di assistenza cronica. Questa nuova generazione di modelli di assistenza cronica prende in considerazione anche i determinanti della salute e si concentra su un sistema di interventi coordinati tra diversi tipi, livelli e impostazioni dell' assistenza, includendo la promozione della salute, la prevenzione, lo screening e la diagnosi precoce, la gestione di casi, la riabilitazione e le cure palliative.

\section{Modelli di assistenza integrati organizzati per anziani e fragili}

Rappresentano un altro specifico gruppo di iniziative. Si distinguono per l'elevata specificità delle risposte individuali del servizio che guidano la progettazione dei modelli di assistenza e la misura in cui l'assistenza richiede l'integrazione tra servizi sanitari e servizi sociali. ${ }^{5}$ PRISMA è un modello canadese progettato per integrare l'assistenza in comunità a soggetti con disabilità da moderata a grave. Lo scopo del modello è preservare l'autonomia funzionale degli individui. Il modello è progettato per servire da punto di accesso unico al sistema e per coordinare l'assistenza attraverso una rete di diversi fornitori. Il case management e le tecnologie informative sono componenti essenziali per il coordinamento. Secondo il modello, l'integrazione viene raggiunta attraverso una commissione congiunta di assistenza sanitaria e sociale. La commissione definisce la strategia, assegna le risorse alla rete e gestisce i gruppi di fornitori. Una valutazione dell'impatto ha dimostrato che il PRISMA che il modello è stato in grado di mantenere la stabilità funzionale degli individui, inoltre ha ridotto l'entità dei bisogni non soddisfatti, l'onere gravante sui caregiver e il rischio di ricoveri ospedalieri. Per superare la frammentazione delle cure per le persone anziane, il distretto di Torbay in Inghilterra ha istituito cinque team integrati di assistenza sanitaria e sociale. Le squadre sono organizzate secondo i principi territoriali e sono allineate con le pratiche generali nelle stesse località. Il modello si focalizza sui pazienti anziani con multimorbidità, che richiedono un supporto intensivo. Il modello impiega coordinatori di assistenza sanitaria e sociale, che fungono da punto di contatto unico all'interno di ciascuna squadra. Come nel PRISMA, il Torbay Care Trust prevede un consiglio congiunto di assistenza sanitaria e sociale con budget in comune. I budget sono assegnati in base alle esigenze della popolazione e non sono limitati a determinati tipi di assistenza. La pianificazione proattiva delle dimissioni e l'assistenza integrata hanno permesso al modello di Torbay di ottenere la riduzione della durata media della degenza ospedaliera e delle riammissioni ospedaliere.

\section{Modelli malattia-specifici}

Alcuni paesi hanno sviluppato modelli volti a fornire una migliore integrazione delle cure per le persone con determinate malattie e condizioni a lungo termine come il diabete mellito, le malattie cardiovascolari, la BPCO e l'asma bronchiale. ${ }^{5}$

Chains of care è un modello di assistenza integrato sviluppato in Svezia con l'obiettivo di collegare l'assistenza primaria, ospedaliera e comunitaria attraverso 
percorsi integrati basati su accordi locali con gli erogatori. Tipiche catene di cura includono l'elemento di screening in un centro di cure primarie, i piani di trattamento sviluppati nel centro specialistico e la riabilitazione fornita nella comunità. Accordi contrattuali e allineamento degli incentivi che consentono un uso efficiente delle risorse sono caratteristiche distintive del modello svedese.

Le reti cliniche sono state sviluppate in Scozia sotto la supervisione di gruppi collegati di professionisti della salute e organizzazioni di cure primarie, secondarie e terziarie, che lavorano in modo coordinato, non vincolati dai confini professionali e sanitari esistenti per garantire un'equa fornitura di servizi di alta qualità e clinicamente efficaci. Il modello è un'integrazione virtuale che mette i pazienti al centro per migliorare l'accesso ai servizi e diminuire le variazioni nella qualità dell'assistenza. La gestione attiva dei pazienti è stata raggiunta garantendo un uso più efficiente della forza lavoro sanitaria.

\section{Programmi di gestione delle malattie}

Si tratta del primo sforzo globale per attuare un programma di assistenza integrata e contrastare la frammentazione e la mancanza di coordinamento tra $\mathrm{i}$ diversi livelli del sistema sanitario tedesco e risalgono al 2002. I programmi di gestione delle malattie (DMP) sono programmi standardizzati a livello nazionale per individui con condizioni croniche, introdotti su un quadro normativo top-down. L'Ufficio federale delle assicurazioni li definisce trattamento coordinato e cura dei pazienti durante l'intera durata di una malattia [cronica] oltre i confini tra i fornitori e sulla base di prove scientifiche e aggiornate. I DMP sono stati ideati per promuovere i principi del miglior trattamento basato sull'evidenza, la promozione dell'erogazione dei servizi attraverso i livelli di cura, l'autogestione dei pazienti e l'introduzione di nuovi meccanismi di garanzia della qualità. L'iscrizione ai DMPs per pazienti e professionisti avviene su base volontaria. I pazienti arruolati svolgono un ruolo attivo nella formulazione e adesione agli obiettivi del trattamento sulla base di un processo decisionale condiviso. Sono inoltre tenuti a partecipare a programmi di educazione e autogestione specifici per la malattia. I fornitori partecipanti devono conformarsi a requisiti di formazione e infrastruttura definiti. I medici di base di solito fungono da coordinatori di cura assicurando che il trattamento e le vie del paziente siano coerenti con le linee guida del DMP basate sull'evidenza. La partecipazione al DMP richiede che i fornitori partecipino attivamente a circoli di qualità e frequentino regolarmente corsi di formazione medica continua. I DMP hanno migliorato il ruolo dei medici generici nell'assistenza cronica e hanno contribuito a una chiara definizione dei ruoli dei fornitori attraverso il continuum delle cure.

\section{Modelli basati sulla popolazione}

Kaiser Permanente (KP) è una delle più grandi organizzazioni di assistenza sanitaria negli Stati Uniti, con oltre 9,6 milioni di membri in otto regioni del paese. KP è un sistema virtualmente integrato costituito da tre entità correlate: un piano sanitario senza scopo di lucro che sostiene i rischi assicurativi (Kaiser Foundation Health Plan), gruppi medici autogestiti (gruppi di medici Permanent) e un'organizzazione ospedaliera non profit (Kaiser Foundation Hospitals). I gruppi medici e gli ospedali della Fondazione Kaiser condividono un budget globale. Tutte e tre le componenti sono reciprocamente esclusive verso l'acquisto e la fornitura di servizi e sono unificate da una missione comune, che rappresenta una miscela di integrazione sistemica e normativa. Il modello di assistenza integrata KP si basa sulla stratificazione della popolazione $^{5}$ e sulla fornitura di diversi tipi di servizi in base alle esigenze. Nel modello KP, la popolazione riceve servizi di promozione e prevenzione allo scopo di controllare l'esposizione ai fattori di rischio; la maggior parte dei pazienti con assistenza cronica riceve supporto per l'autogestione della propria malattia e i pazienti ad alto rischio ricevono la servizi di gestione delle malattie o dei casi, che combinano l'autogestione e l'assistenza professionale. Il modello di stratificazione è presentato nella famosa Piramide di Kaiser.

L'integrazione all'interno del modello KP è focalizzata sull'assistenza cronica e sulla pratica multispecialistica, piuttosto che sull'assistenza primaria e secondaria. I componenti principali del modello KP mettono l'accento sulla prevenzione, il supporto all'autogestione, la gestione delle malattie e la gestione dei casi per i pluripatologici. Un componente cruciale che ha definito il successo del modello di assistenza integrata KP è che tutte le entità all'interno del gruppo $\mathrm{KP}$ sono reciprocamente responsabili per i risultati clinici e la qualità percepita del paziente e gli incentivi del fornitore sono legati alla qualità dell'assistenza e alla soddisfazione del paziente. A questo proposito un episodio di ammissione ospedaliera acuta o riammissione è visto come fallimento dell'intero sistema. La gestione attiva dei pazienti negli ospedali è assicurata attraverso percorsi e protocolli clinici chiaramente definiti e basati sull'evidenza. Il modello KP è stato innovativo nella creazione di nuove figure professionali: gli hospitalist, i responsabili delle dimissioni, i vari profili infermieristici e i coordinatori delle cure, consentendo un uso efficiente delle risorse umane e transizioni continue di assistenza tra le varie impostazioni. Un altro elemento cruciale del modello KP è il superamento dell'asimmetria e della frammentazione delle informazioni tra i fornitori attraverso l'introduzione del proprio ampio sistema informativo - Health Connect Program - permette ai fornitori all'interno del gruppo KP di accedere alle cartelle dei pazienti sia am- 
bulatoriali che non, oltre a facilitare l'autogestione dei pazienti consentendo ai pazienti di accedere ai loro registri, fissare appuntamenti e ordinare le ricariche dei farmaci. L'adozione delle strategie di governance clinica e la costante attenzione al miglioramento delle prestazioni consentono al modello KP di mantenersi ed evolvere nel tempo.

Sempre negli Stati Uniti, la Veterans Health Administration (VA) è un sistema sanitario che fornisce servizi integrati a persone anziane con patologie croniche. La VA impiega medici, possiede e gestisce ospedali, studi medici e gestisce i servizi all'interno della sua rete. Nata dalla trasformazione di un sistema ospedaliero, la VA attualmente è composta da 21 reti di servizi integrati su base regionale. I cambiamenti strutturali sono stati guidati dal presupposto che i guadagni in termini di efficacia ed efficienza possono essere raggiunti attraverso un migliore coordinamento tra le strutture, la sinergia di risorse e la fornitura di assistenza nelle impostazioni più appropriate. Le risorse ricevute dal governo federale non vengono assegnate alle strutture ma piuttosto alle reti: un meccanismo attraverso il quale si ottengono l'integrazione dei servizi e la responsabilità condivisa. Una caratteristica distintiva del sistema VA è la rinomata cultura della misurazione e del reporting che ha supportato i meccanismi di responsabilità e miglioramento continuo delle prestazioni. Le misure sono determinate da gruppi di indicatori che sono strettamente monitorati in ciascuna delle reti. Il modello VA ha fatto un grande sforzo nell'organizzare i processi di cura intorno alle esigenze dei pazienti e nell'abilitare l'autogestione dei pazienti attraverso investimenti in tecnologie informatiche di supporto. Gran parte delle trasformazioni sono state ottenute in virtù della promozione della governance clinica e della ricerca sui servizi sanitari del VA.

\section{Strategia per affrontare la sfida della cronicità nei Paesi Baschi}

Per ottenere l'assistenza integrata stati utilizzati due approcci. Un approccio di implementazione dal basso verso l'alto incentrato sull'integrazione clinica e funzionale che ha promosso il coordinamento dei processi di cura tra assistenza primaria e secondaria. È stata realizzata un'integrazione organizzativa combinando strutture ospedaliere e di assistenza primaria in un'unica organizzazione - Integrated Healthcare Organisations (IHO). Attualmente, il Paese basco conta 13 IHO con aree di utenza da 30.000 a 400.000 persone. L'attivazione simultanea di tutti i livelli del sistema e lo sviluppo di nuove professioni che facilitano l'erogazione delle cure integrate sono caratteristiche importanti di questo modello. Gli IHO di Bidasoa hanno creato, ad esempio, le Unità di Continuità di Cura (CCU) per il trattamento di pazienti ad alto rischio con co- e multimorbidità. Le CCU usano la continuità individuale dei piani di assistenza per migliorare il coordinamento tra i fornitori. Le CCU sono dotate di internisti di riferimento designati responsabili dell'ammissione e della stabilizzazione dei pazienti cronici e degli infermieri di collegamento che sostengono la dimissione e il passaggio dei pazienti dall'ospedale a casa, dove saranno poi seguiti dal loro medico di famiglia. Gli internisti di riferimento lavorano in stretta collaborazione con i medici di base nella pianificazione delle cure al di fuori degli episodi acuti. L'educazione del paziente all'autogestione, le cartelle cliniche condivise e un nuovo sistema di informazioni sanitarie sviluppato insieme agli utenti rappresentano elementi di successo che hanno facilitato il coordinamento tra i diversi erogatori. Il monitoraggio di specifici indicatori quali ricoveri ospedalieri e riammissioni per condizioni gestibili in ambito ambulatoriale, ha permesso di dimostrare impatti positivi su outcome clinici ed economici. Questi ultimi sono stati una componente essenziale del programma per superare la resistenza delle parti interessate e assicurare la sostenibilità dell'iniziativa.

\section{La realtà del nostro paese}

L'Italia ha definito come il Servizio Sanitario Nazionale intende occuparsi delle persone che soffrono di malattie croniche nel Piano Nazionale delle Cronicità, pubblicato nel $2016 .{ }^{1}$

Il Piano si compone di due parti: la prima contiene gli indirizzi generali per la cronicità, mentre nella seconda si trovano approfondimenti su patologie con caratteristiche e bisogni assistenziali specifici.

Nella prima parte vengono indicati la strategia complessiva e gli obiettivi di Piano, proposte alcune linee di intervento ed evidenziati i risultati attesi, attraverso i quali migliorare la gestione della cronicità nel rispetto delle evidenze scientifiche, dell'appropriatezza delle prestazioni e della condivisione dei PDTA.

Nella seconda parte il Piano individua un primo elenco di patologie croniche da gestire attraverso criteri quali la rilevanza epidemiologica, la gravità, l'invalidità, il peso assistenziale ed economico, la difficoltà di diagnosi e di accesso alle cure.

Le leve sono la informazione della popolazione e la formazione degli operatori.

Attraverso l'Intesa di settembre 2016 tutte le Regioni e Province autonome si sono impegnate a recepire il documento con propri provvedimenti e a dare attuazione ai contenuti del Piano nei rispettivi ambiti territoriali.

Sei gli aspetti chiave del piano e cioè aderenza, appropriatezza, prevenzione, cure domiciliari, formazione/educazione/empowerment, conoscenza e competenza.

Sette gli aspetti trasversali dell'assistenza: disu- 
guaglianze sociali, fragilità e vulnerabilità; diffusione delle competenze, formazione, sostegno alla ricerca; appropriatezza nell'uso delle terapie e delle tecnologie; sanità digitale; umanizzazione delle cure; ruolo delle associazioni di tutela delle persone con malattie croniche e delle loro famiglie; ruolo delle farmacie.

Dieci le patologie sulle quali si inizia a lavorare attraverso il Piano: malattie renali croniche e insufficienza renale; artrite reumatoide e artriti croniche in età evolutiva; rettocolite ulcerosa e malattia di Crohn; insufficienza cardiaca cronica; malattia di Parkinson e parkinsonismi; Broncopneumopatia cronica ostruttiva e insufficienza respiratoria cronica; insufficienza respiratoria cronica in età evolutiva; asma in età evolutiva; malattie endocrine croniche in età evolutiva; malattie renali croniche in età evolutiva.

Umbria, Puglia, Lazio, Emilia Romagna, Marche hanno recepito con proprio atto il PNC, mentre la Regione Toscana, sta lavorando a IDEA: Incontri $D i$ Educazione all'Autogestione delle malattie croniche. Approvazione e destinazione risorse. Il Piemonte ha un iter approvativo ancora in corso. La Lombardia ha un suo Piano Regionale della Cronicità e Fragilità e successivi provvedimenti attuativi. ${ }^{7}$

\section{Quali strumenti disponibili oggi in Italia?}

Fra gli strumenti di gestione delle cronicità un ruolo rilevante è riservato alla redazione e applicazione dei percorsi di presa in carico, i cosiddetti percorsi diagnostico-terapeutico assistenziali (PDTA) nei quali l'assistenza primaria rappresenta il punto chiave, la proattività e l'investimento per quanto riguarda l'empowerment del paziente e della famiglia.

Il Piano nazionale cronicità ha dato alle Regioni un manuale operativo che riguarda per ora 6 patologie ed individua degli indicatori di processo e di esito sui quali le Regioni dovranno lavorare nel definire $\mathrm{i}$ PDTA clinici. ${ }^{1}$

A fine 2017 risultavano definiti a livello regionale 116 PDTA per le patologie croniche: 26 per patologie cardiovascolari, 24 per quelle neurologiche, 12 per le malattie respiratorie, 10 per quelle reumatiche, 9 per le patologie endocrine, 8 per i percorsi riabilitativi e gastrointestinali, 5 per le patologie psichiatriche e per quelle genetiche, 4 per le renali ed oculari, 5 per altre patologie. ${ }^{1}$

Piemonte, Toscana e Puglia sono le uniche tre regioni che hanno attivato i PDTA per il Parkinson. Questi sono simili nella struttura, ma si differenziano nell'identificazione del bisogno assistenziale del malato di Parkinson, nelle indagini diagnostiche proposte, e soprattutto negli indicatori, questi ultimi riguardano il costo sociale della malattia. ${ }^{1}$

Regione Liguria prima di realizzare PDTA regionali ha istituito i DIAR (dipartimenti interaziendali regio- nali) quali strumento organizzativo della programmazione strategica, dell'integrazione inter-Aziendale, dell'integrazione dei diversi livelli di assistenza delle attività sanitarie e sociosanitarie. Essi rappresentano soprattutto la risposta organizzativa per superare la frammentazione assistenziale e l'autoreferenzialità, a favore della presa in carico globale del paziente, della continuità delle cure e della condivisione ed uniforme applicazione di percorsi evidence-based. ${ }^{8}$

I DIAR attivati finora sono: oncoematologico, emergenza urgenza, neuroscienze, chirurgico e cardiotoracovascolare. All'interno di questi DIAR sono in fase di avanzata produzione PDTA che saranno deliberati dalla giunta regionale e diventeranno documenti guida per la presa in carico omogenea dei pazienti in tutta la regione. ${ }^{8}$

In particolare nei PDTA relativi alla cronicità è enfatizzata la presa in carico continuativa del paziente cronico con una forte condivisione del percorso diagnostico terapeutico fra il setting ospedaliero e quello territoriale.

La gestione ottimale delle malattie croniche nei pazienti ultra-quarantenni affetti patologie croniche come ipertensione arteriosa, scompenso cardiaco, broncopneumopatia cronico-ostruttiva e diabete si avvale anche di un altro strumento messo già in atto da alcune regioni (Lombardia, Puglia, Liguria, ecc.), il PAI o Piano Assistenziale Individuale. Un numero consistente di pazienti potranno essere presi in carico dai Medici di medicina generale con l'aiuto di un infermiere professionale e un collaboratore di studio attraverso la sottoscrizione del Piano Assistenziale Individuale, al fine di garantirne la presa in carico effettiva. L'infermiere svolge funzione di Care Manager mentre il collaboratore di studio garantisce la gestione del percorso anche attraverso la prenotazione in agende dedicate. Il Piano Assistenziale Individualizzato definisce gli obiettivi ed $\mathrm{i}$ risultati attesi in termini di mantenimento o miglioramento dello stato di salute della persona con patologia cronica e individua il livello di complessità, la durata dell'intervento, le prestazioni sociosanitarie che devono essere erogate, compatibilmente con le risorse a disposizione; nel PAI sono individuate le responsabilità dei soggetti/strutture coinvolte e gli operatori che seguono il paziente. Il piano di assistenza tiene conto anche della situazione socio-ambientale del paziente, inclusa la presenza di relazioni familiari e di caregiver di riferimento, elementi questi che, per le persone non autosufficienti, concorrono alla scelta di una assistenza di tipo domiciliare o residenziale. Infine, è prevista la rivalutazione periodica e sistematica, da parte di un'equipe multi-professionale, delle condizioni complessive della persona e del suo stato di salute/benessere al fine di confermare o adeguare il PAI in relazione all'evoluzione dei bisogni assistenziali.

Un altro strumento di coordinamento delle cure e so- 
prattutto di continuità assistenziale declinato dal piano nazionale cronicità è l'Assistenza domiciliare integrata (ADI). Essa la modalità privilegiata di intervento per rispondere ad esigenze complesse di persone non autosufficienti, poiché permette l'erogazione di prestazioni sanitarie e sociosanitarie anche di elevata complessità ed intensità assistenziale favorendo il mantenimento del paziente nel contesto abitativo e familiare.

L'assistenza domiciliare integrata si realizza tramite l'erogazione coordinata e continuativa di un insieme integrato di prestazioni, sia sanitarie (ad es. mediche, infermieristiche e riabilitative, assistenza farmaceutica e accertamenti diagnostici), sia socio-assistenziali (cura della persona, fornitura dei pasti, cure domestiche) da parte di diverse figure professionali. La responsabilità clinica è attribuita al medico di medicina generale, o nei casi previsti, al medico competente per la terapia del dolore e le cure palliative. L'attivazione dell'ADI richiede la presa in carico della persona, la preliminare valutazione multidimensionale del bisogno e la definizione di un Piano di cura personalizzato, con individuazione degli obiettivi di cura e dei tempi di recupero, delle tipologie di intervento e della frequenza degli accessi. I servizi domiciliari sono così chiamati ad orientare la loro azione sulla base dei seguenti criteri: mantenere la persona nel suo ambiente di vita, supportare la famiglia, offrire soluzioni alternative quando tale supporto manca o viene meno, assicurare continuità assistenziale mediante dimissioni programmate dall'ospedale per acuti, così come dalle strutture riabilitative (post-acute ed estensive) o dalle Residenze sanitarie assistenziali.

Se il paziente è tenuto all'interno si un sistema proattivo di Sanità questo può fare crollare i ricoveri dei frequent user e i costi del sistema. In questo scenario è importante innovare ruoli e funzioni e lavorare in équipe ed è fondamentale la figura del Care Manager e il nuovo ruolo degli infermieri, ma anche la stratificazione dei pazienti e i sistemi informativi per la cui attivazione il professionista sanitario deve collaborare attivamente, affinché siano uno strumento efficace per la continuità assistenziale.

In quest'ottica, elemento essenziale per la concreta presa in carico delle cronicità prevista dal Piano Nazionale è la sono essenziali per fornire un opportuno supporto alla condivisione/circolazione dei dati clinici per ottimizzare il percorso di cura, evitare la inutile ripetizione di procedure diagnostiche e terapeutiche, consentire il monitoraggio continuo da parte dei vari attori assistenziali, la programmazione degli interventi e la gestione personalizzata dei pazienti.

Sedici Regioni, oltre alla Provincia Autonoma di Bolzano, stanno lavorando all'implementazione del fascicolo sanitario elettronico (FSE), ma solo 11 aderiscono all'interoperabilità, stando agli aggiornamenti della Agenzia per l'Italia Digitale. Ad oggi sono stati attivati circa 11 milioni e mezzo (11.484.678) di fascicoli sanitari. Nei primissimi mesi del 2018 le Regioni che ne hanno attivato di più sono: P.A. Trento, Friuli Venezia Giulia, Lombardia, Toscana e Valle d'Aosta. Questa implementazione tecnologica presuppone lo stanziamento di risorse per la sua realizzazione. Sebbene il Piano non abbia fondi ad hoc per la sua messa in atto, nelle sue premesse si sottolinea che è opportuno accedere ad altre fonti di finanziamento per la diffusione di strumenti e tecnologie ICT a supporto della cronicità: tra di esse i fondi del PON GOV Cronicità (le risorse per l'ICT in sanità) 2017-2023 pari a 21.154.946,00 euro.

Le liste di attesa rappresentano un fenomeno percepito dai cittadini e dai pazienti come una forte criticità dei sistemi sanitari, in quanto compromette l'accessibilità, la fruibilità dell'offerta sanitaria e la compliance ai PDTA. L'abbattimento dei tempi di attesa per le prestazioni sanitarie è uno degli obiettivi prioritari del SSN e l'erogazione dei servizi entro i tempi appropriati rispetto alla patologia e alle necessità delle cure, rappresenta una componente strutturale dei LEA. Gli obiettivi strategici devono essere quelli di promuovere la capacità del sistema di intercettare il reale bisogno di salute, di ridurre l'inappropriatezza e rendere compatibile la domanda con la garanzia dei LEA.

Fornire gratuitamente un numero crescente di prestazioni sanitarie a una quantità di persone sempre maggiore, nei tempi previsti dalla legge e con risorse sempre più limitate in rapporto alla richiesta, è un'impresa difficilmente raggiungibile soprattutto in aree vaste; si creano pertanto liste di attesa spesso by-passate dalle prestazioni private a pagamento e a carico del singolo cittadino. A farne le spese sono spesso le categorie più fragili (anziani, malati polipatologici e pensionati monoreddito) ed è indispensabile che le aziende sanitarie provvedano ad implementare modelli strutturati di presa in carico della criticità nell'ottica della massima appropriatezza, efficacia ed efficienza.

CONSENSO (COmmunity Nurse Supporting Elderly iN a changing SOciety) è progetto europeo che si pone l'obiettivo di favorire l'invecchiamento sano e attivo della popolazione residente in zone disagiate, permettendo agli anziani di restare a casa loro attraverso interventi incentrati sulla figura dell'infermiere di comunità. L'infermiere di famiglia e di comunità è una figura chiave in grado di aiutare e sostenere le persone anziane e le loro famiglie e facilita l'accesso ai servizi disponibili sul territorio, attiva gli interventi necessari - sia direttamente sia in collaborazione con il medico di medicina generale - dialoga, informa e consiglia gli anziani sui corretti stili di vita, con particolare riferimento ad alimentazione e attività fisica. L'adesione a questo progetto soprattutto nelle zone disagiate rappresenta realmente uno strumento impor- 
tante per il coordinamento delle cure e la continuità assistenziale nella gestione delle malattie croniche.

\section{Quali sono i potenziali strumenti fruibili in futuro?}

Il piano cronicità del Ministero del 2016 prevede una serie di interventi che hanno bisogno di tempo ed investimenti per giungere alla loro completa realizzazione.

Uno di questi è quello legato alla sanità digitale che sta muovendo i primi passi in larga scala proprio questi anni. L'obiettivo della sanità digitale è quello di promuovere l'impiego di modelli, tecniche e strumenti informatci nella gestione della cronicità al fine di garantire continuità e migliore qualità dell'assistenza, migliore efficacia, efficienza e appropriatezza.

Le linee di intervento proposte sono: i) sperimentare modelli di assistenza che riescano a coniugare soluzioni tecnologiche con i bisogni di salute del paziente (Teleassistenza domiciliare, Teleconsulto specialistico, telemonitoraggio medicale, Telesorveglianza, Telecontrollo, Telesoccorso, Teleallarme); ii) analizzare modelli, processi e modalità di integrazione dei servizi di telemedicina nella pratica clinica; iii) diffondere nei cittadini, nei pazienti, negli operatori e nelle istituzioni la cultura della telemedicina; iv) potenziare percorsi di formazione e aggiornamento continuo per gli operatori dei servizi di telemedicina; v) produrre studi di fattibilità e di analisi costo-beneficio e condividere le esperienze in telemedicina.

Le tecnologie della sanità digitale (eHealth) possono risultare di grande supporto nell'implementazione di modelli di gestione della cronicità basati sul Chronic Care Model (CCM). È possibile ipotizzare un modello concettuale di CCM rafforzato dalle tecnologie eHealth (eCCM) che non solo preveda un contributo della eHealth sulla messa in azione di ogni componente del CCM, ma possa fornire un supporto fondamentale nell'ambito dell'educazione e della formazione del paziente, portando ad una componente aggiuntiva del CCM (eHealth education).

Il coinvolgimento attivo del cittadino in un'ottica di patient empowerment, richiede la presenza di una infrastruttura di servizi online che consentano al cittadino di entrare in rete favorendo la comunicazione e lo scambio di informazioni tra il singolo cittadino e i propri familiari, gli operatori sanitari ed altri cittadini.

Le tecnologie e-Health sono già in grado di supportare la creazione di questa infrastruttura digitale di servizi sanitari innovativi (e-care) mettendo a disposizione del cittadino strumenti tecnologici che lo aiutino e lo accompagnino nella gestione della propria salute nella vita di tutti i giorni, con facilità, attraverso vari dispositivi (computer, smartphone, tablet, sensori, ecc), ovunque esso si trovi (casa, lavoro, ferie), e nel momento del bisogno. In particolare, la grande diffusione dei dispositivi mobile ed il crescente sviluppo di sensori indossabili rappresentano grandi potenzialità per supportare l'implementazione di nuovi modelli di assistenza focalizzati sulla prevenzione e sul miglioramento qualità della vita e su l'empowerment dei cittadini/pazienti. Di fatto, il paziente attraverso l'utilizzo di applicazioni mobile certificate può raccogliere dati strutturati sul proprio stato di salute (es. sintomi, farmaci assunti, ecc.), mentre attraverso l'uso di sensori connessi all'applicazione mobile può tenere traccia con facilità dei parametri vitali (es. glicemia, peso, movimento, ecc). L'uso di questi dati, generati dal paziente stesso nei periodi che intercorrono fra le visite, su base quotidiana e in modo semplice, offre un'opportunità unica di monitorare da remoto e in tempo reale lo stato di salute, migliorando la qualità dell'assistenza e riducendo i costi di gestione del malato cronico.

L'utilizzo di sistemi basati su Personal Health Record, sistemi a supporto delle decisioni (Clinical Decision Support System) e tecnologie persuasive consentiranno di creare delle piattaforme più evolute di tele-salute in grado di migliorare ulteriormente il monitoraggio remoto e la self-care dei malati cronici. Inoltre sistemi di modellazione di percorsi di cura e di gestione multiutente del paziente cronico basati su differenti basi di conoscenza (medica, organizzativa), potranno facilitare il coordinamento e la collaborazione degli attori coinvolti nel processo di cura supportando l'implementazione di percorsi assistenziali personalizzati, integrati e multidisciplinari.

La tecnologia può offrire soluzioni anche all'annoso problema delle liste di attesa che impattano negativamente sulla gestione ottimale delle malattie croniche, come recentemente riportato dalla nostra Azienda su Politiche Sanitarie, in press da FIASO (Federazione delle Aziende Sanitarie e Ospedaliere). Il tentativo di rispettare le classi di priorità d'accesso previste dalla normativa nazionale (priorità $\mathrm{B}=$ breve prestazione da erogare entro i 10 gg; priorità $\mathrm{D}=$ differita prestazione da erogare entro i 30/60 gg; priorità $\mathrm{P}=$ programmata da erogare entro 1 anno) può essere messo in atto attraverso azioni e tecnologie che non sono particolarmente difficili da applicare nelle varie realtà aziendali: i) sensibilizzazione dei cittadini, mediante una campagna di comunicazione, sull'importanza di rispettare o disdire per tempo gli appuntamenti prenotati al fine di consentire ad altre persone l'accesso alle prestazioni, evitare lo spreco delle risorse e, non ultimo, ottimizzare il lavoro dei professionisti con coinvolgimento del territorio (MMG, farmacie, distretti); ii) sensibilizzazione dei medici di medicina generale attraverso una serie di incontri su temi di appropriatezza prescrittiva e realizzazione di PDTA su patologie più frequenti al fine di disegnare percorsi condivisi che permettano di erogare 
prestazioni al di fuori delle liste di attesa; iii) recall telefonico informatizzato: una settimana prima della visita o dell'esame può effettuata una chiamata al cittadino per ricordare la prestazione prenotata e chiederne conferma o disdetta, attraverso un risponditore automatico che permette l'eventuale annullamento al momento del contatto telefonico; gli appuntamenti disdetti rientrano in tal modo nella disponibilità di altri cittadini; iv) numero verde: dedicato alle segnalazioni dei cittadini che ritengano non conforme il tempo di attesa della loro prenotazione. L'azienda effettua una presa in carico, mediante l'ascolto della persona, per individuare il problema e le sue reali necessità; v) sistema Agende Dinamiche: attivazione di un sistema informatico che consente di rendere disponibili, e quindi prenotabili, gli appuntamenti originariamente riservati a priorità $\mathrm{B}$ e $\mathrm{D}$ non utilizzati.

Infine, è atteso che anche il farmacista, storicamente legato alla dispensazione di farmaci dietro presentazione di una prescrizione medica, partecipi in fututo a modelli di cure integrate per la cronicità, attraverso la concreta realizzazione di quella che viene definita farmacia dei servizi. La capillare diffusione sul territorio, l'ampio orario di apertura (secondo solo ai pronto soccorso e alle strutture di degenza) e la relazione di convenzione con il sistema sanitario nazionale sono i presupposti di questa partecipazione. A tal proposito possiamo citare uno studio osservazionale no-profit sul ruolo del farmacista territoriale e della farmacia dei servizi nel case management del paziente diabetico, in atto in Campania. ${ }^{9}$ La farmacia, dotata di telecardiologia, POCT per gli esami ematici, esame robotizzato del fondo oculare e rilevazione dell'indice di windsor per lo screening delle arteriopatie e collegata in rete con MMG e specialisti, diventa una smart clinic presso cui ogni paziente diabetico può seguire puntualmente il suo PDTA senza file, senza assenze al lavoro e senza allontanarsi dalla località di residenza/domicilio.

\section{Conclusioni}

La cronicità è associata al declino di aspetti della vita come l'autonomia, la mobilità, la capacità funzionale e la vita di relazione con conseguente aumento di stress psicologico, ospedalizzazioni, uso di risorse (sanitarie, sociali, assistenziali) e mortalità. Secondo dati ISTAT, in Italia due milioni 600 mila persone vivono in condizione di disabilità $(4,8 \%$ della popolazione, totale, $44,5 \%$ nella fascia di età $>80$ anni) e ciò testimonia una trasformazione radicale nell'epidemiologia del nostro Paese.

In Europa si stima che le malattie croniche, nel complesso, siano responsabili dell' $86 \%$ di tutti i decessi e di una spesa sanitaria valutabile intorno ai 700 miliardi di Euro per anno.

Per vincere la sfida contro queste nuove epidemie è necessario coinvolgere e responsabilizzare tutte le componenti, dalla persona con cronicità al macrosistema-salute, formato non solo dai servizi, ma da tutti gli attori istituzionali e sociali che hanno influenza sulla salute delle comunità e dei singoli individui.

Si deve partire da una profonda riflessione sulle tendenze e sugli indirizzi dei servizi e dei professionisti, promuovendo una nuova cultura che dovrà impregnare, al di là delle differenze di ruolo, tutte le scelte di politica sanitaria, le strategie assistenziali, la tipologia dell'organizzazione, l'assetto operativo delle strutture e la conseguente offerta dei servizi.

Questo è il presupposto fondamentale per la gestione efficace della cronicità, perché è da tali premesse che originano le scelte e gli indirizzi strategici, si decidono le caratteristiche dell'offerta sanitaria, si promuovono i comportamenti dei protagonisti gestionali e professionali del mondo assistenziale.

\section{Bibliografia}

1. Direzione Generale Programmazione Sanitaria del Ministero della Salute, Piano Nazionale Cronicità, Accordo tra lo Stato, le Regioni e le Province Autonome di Trento e di Bolzano del 15 settembre 2016.

2. D.C.P.M. 29 novembre 2001 Definizione dei livelli essenziali di assistenza. Pubblicato in GAzz. Uff. del 8 febbraio 2002 n.33.

3. D.C.P.M. 12 gennaio 2017. Definizione e aggiornamento dei livelli essenziali di assistenza, di cui all'articolo 1 , comma 7 , del decreto legislativo 30 dicembre 1992, n. 502. Pubblicato in Gazz. Uff. n.65 del 18 marzo 2017 Suppl. Ord.n.15.

4. Baxter S, Johnson M, Chambers D, et al. The effects of integrated care: a systematic review of UK and international evidence. BMC Health Serv Res 2018;18:350.

5. WHO Integrated care models: an overview. Working document; 2016. Available from: http://www.euro.who.int/ data/assets/pdf_file/0005/322475/Integrated-care-models-overview.pdf

6. Action Plan for prevention and control of non-communicable diseases 2012-2016"; "Resolution EUR/RC 61/R3 on the action plan for implementation of the European strategy for the prevention and control of non-communicable diseases 2012-2016. http://www.euro.who.int/en/ health-topics/noncommunicable-diseases/cancer/publications/2012/action-plan-for-implementation-of-the-european-strategy-for-the-prevention-and-control-of-noncom municable-diseases-20122016

7. "Indirizzi per la presa in carico della cronicità e della fragilità in Regione Lombardia 2016-2018". http://www. lombardiasociale.it/wp-content/uploads/2016/01/DGR4662-23.12.15-Piano-Cronicità.pdf

8. Piano Socio Sanitario Regionale per il triennio 2017/2019 Regione Liguria. http://www.alisa.liguria.it/index.php? option=com_content\&view=article\&id=28\&Itemid $=173$

9. ClinicalTrials.gov. New patient support program in type 2 diabetes. Available from: https://clinicaltrials.gov/ct2/ show/NCT03752567 


\title{
La mancata pianificazione dei fabbisogni di medici e di specialisti in Italia nel Servizio Sanitario pubblico. Quali conseguenze in rapporto all'invecchiamento della popolazione?
}

\author{
Domenico Montemurro, ${ }^{1}$ Matteo D’Arienzo,${ }^{2}$ Chiara Rivetti, ${ }^{3}$ Elena Marcante, ${ }^{4}$ Fabio Ragazzo, ${ }^{5}$ \\ Pierino Di Silverio, ${ }^{6}$ Andrea Rossi, ${ }^{7}$ Carlo Palermo ${ }^{8}$
}

${ }^{1}$ Dirigente di Organizzazione Sanitaria, ULSS 6 EUGANEA, Presidio Madre Teresa di Calcutta; ${ }^{2}$ Dirigente Medico Pronto Soccorso e Medicina d'Urgenza, Azienda Ospedaliero-Universitaria di Modena; ${ }^{3} \mathrm{SC}$ Medicina Interna, Ospedale di Chieri, ASL TO 5; ${ }^{4}$ Medico in Formazione Specialistica in Igiene e Sanità Pubblica, Università degli Studi di Padova; ${ }^{5}$ Dirigente Medico, Medicina Generale 1, Ospedale San Bortolo Vicenza, AULSS 8 Berica; ${ }^{\circ}$ Dirigente Medico, Centro Regionale Trapianti Campania; ${ }^{7}$ Azienda Ospedaliera Universitaria Integrata di Verona, Healthy Aging Center Verona; ${ }^{8}$ Direttore Dipartimento di Medicina Interna e Specialistica, Azienda USL Sud Est Toscana, Arezzo, Italia

\section{Invecchiamento della popolazione italiana e carenza di medici specialisti}

L'invecchiamento della popolazione rappresenta uno dei fenomeni più rilevanti nei paesi industrializzati, ove circa un quarto della popolazione ha più di 65 anni di età, con un incremento dei soggetti con età over 80 ancor più elevato di quello delle altre fasce di età, con significativi riflessi non solo sul mondo sanitario, ma anche su quello socio-assistenziale.

Nel periodo 2003-2008 il tasso di ospedalizzazione degli over 75 anni in Italia è aumentato del 375\%. I pazienti anziani risultano essere quelli con numerosità di gran lunga maggiore in tutte le unità operative delle Aziende Ospedaliere. Tutte le figure specialistiche nel corso del proprio lavoro quotidiano, in Ospedale o sul Territorio, si confrontano con soggetti anziani e/o grandi anziani.

Anche il numero di anziani ultraottantenni sottoposti a interventi chirurgici sta aumentando esponenzialmente negli ultimi anni e nei prossimi decenni si assisterà ad un ulteriore aumento percentuale di interventi chirurgici soprattutto nelle fasce di pazienti più anziani.

Corrispondente: Domenico Montemurro, Via Albere 30, 35043 Monselice (PD), Italia

Tel.: +39.0429.714111.

E-mail: domemontemurro@gmail.com

Articolo pubblicato secondo la Creative Commons Attribution NonCommercial 4.0 License (CC BY-NC 4.0).

CCopyright D. Montemurro et al., 2019

Licensee PAGEPress, Italy

QUADERNI - Italian Journal of Medicine 2019; 7(4):21-25
Uno studio condotto negli Usa ha dimostrato che i pazienti over 75 anni sottoposti a interventi chirurgici nei reparti di chirurgia generale rappresentavano circa il $60 \%$ della popolazione ricoverata. Tra il 2001 e il 2010 si è osservato un aumento del 13\% delle procedure chirurgiche ed è stato stimato un suo ulteriore aumento fino al 28\% nel 2020.

In Italia, l'aumento della popolazione anziana - dovuto ai guadagni in termini di sopravvivenza - e la presenza di generazioni di giovani sempre meno folte conseguenza del continuo calo delle nascite - rendono il nostro paese il secondo più vecchio al mondo, con una stima di 168,7 anziani ogni cento giovani al $1^{\circ}$ gennaio 2018. Questo è quanto evidenzia l'Istat nella 26esima edizione del Rapporto annuale che verte sul tema delle reti e delle relazioni sociali ed economiche.

La tendenza demografica è destinata ad accentuare ulteriormente il processo di invecchiamento: 1'Istat conferma che secondo lo scenario mediano delle previsioni demografiche, tra 20 anni lo squilibrio intergenerazionale sarà ancora più critico, con 265 anziani ogni 100 giovani.

In considerazione della complessità clinica del paziente anziano, emerge la necessità di modelli assistenziali e formativi con una visione patient centered anziché con una visione disease centered. Il modello della presa in carico al posto del modello della cura, che tenga conto delle necessità complessive del paziente anziano, anche nel post ricovero.

I bisogni assistenziali per la popolazione anziana tra ospedale e territorio vanno quindi ridisegnati, così come il numero di specialisti necessari per mantenerne gli standard qualitativi.

Il nostro sistema sanitario rischia di andare incontro nei prossimi anni ad una grave crisi delle proprie risorse professionali mediche. Il pericolo è stato segnalato dall'Anaao Assomed fin dal 2011 (Enrico Re- 
ginato, Carlo Palermo; Sole 24 Ore Sanità n.36). A fronte dell'indifferenza mostrata dai precedenti governi, invischiati in relazioni di potere fondate più su interessi autoreferenziali che su attenzione alle esigenze del Paese, la realtà inesorabilmente sta evidenziando, anno dopo anno, quanto fossero fondate le criticità rilevate, sostenute da molteplici fattori oramai così deteriorati da richiedere urgenti risposte.

Il depauperamento degli organici è certamente conseguente alla crisi economica e all'imposizione del vincolo nazionale della spesa per il personale, perpetrato nel tempo e fissato con la Legge Finanziaria del 2009 al dato del 2004 ridotto dell' $1,4 \%$, che ha avuto come inevitabile conseguenza il mancato rispetto del turnover.

Tuttavia la mancanza di medici specialisti all'interno del SSN e l'accelerazione del loro pensionamento sono realtà che stanno rapidamente assumendo i contorni di una vera emergenza nazionale, cui vanno posti correttivi rapidi ed adeguati per evitare il collasso del sistema stesso.

\section{Carenza di medici specialisti}

Primo fra tutti va ricordato il totale fallimento della programmazione del numero di specialisti per regione e disciplina.

Ogni anno infatti si laureano circa 10.000 medici, ma i contratti per la formazione specialistica nel 2018 sono stati poco meno di 7000, determinando il cosiddetto imbuto formativo, che negli anni ha accumunato in un limbo oltre 10.000 giovani medici, che aumenteranno nei prossimi 5 anni fino ad oltre 20.000 unità senza un forte incremento dei contratti di formazione specialistica. Giovani medici laureati posti tra color che son sospesi destinati a ritentare l'ammissione alle scuole di specialità l'anno successivo o a lasciare il nostro Paese, regalando ad altre nazioni, in particolare Regno Unito, Germania, Svizzera e Francia, l'investimento per la loro formazione scolastica ed universitaria, circa 150.000/200.000€ per medico, il costo di una Ferrari.

A questo aggiungiamo la carenza di vocazioni verso determinate branche specialistiche, testimoniate dal recente censimento ALS (Associazione Liberi Specializzandi), relativo ai contratti di formazione specialistica assegnati all'ottavo scaglione 2018, pubblicate lo scorso ottobre. Da tali dati risulta evidente come, al momento del censimento, alcune specialità chirurgiche risultassero scarsamente appetibili, come ad esempio chirurgia toracica (assegnate il 15,15\% delle borse), chirurgia generale (assegnato il $31,03 \%$ ), chirurgia vascolare (assegnato il $34,48 \%$ ) e ortopedia e traumatologia (assegnato il 47,29\%), Anche per quanto riguarda le specialità legate all'emergenza urgenza va registrata una bassa attrattività: medicina d'urgenza registrava infatti una percentuale di assegnazione del $32,81 \%$ e anestesia e rianimazione del $40,23 \%$. Risultavano invece da tempo saturati al 100\% i posti in chirurgia plastica, dermatologia, oculistica, endocrinologia, pediatria, oftalmologia e cardiologia, discipline che aprono sbocchi di carriera anche sul territorio e nel privato, con prospettiva di maggior guadagno e di una più alta qualità di vita.

Inoltre il peggioramento delle condizioni di lavoro, con aumento dei carichi individuali, associato al mancato rispetto della normativa europea sui riposi ed alimentato da un sentimento di sfiducia rispetto ad un possibile miglioramento della situazione, ha spinto numerosi medici a lasciare gli ospedali pubblici in favore del privato o a emigrare in altre regioni alla ricerca di soddisfazioni professionali ed economiche maggiori. Il fenomeno, inizialmente marginale e $f i$ siologico, sta assumendo ora dimensioni preoccupanti, in particolare in alcune regioni italiane, coinvolgendo in particolare le UU.OO. di Anestesia e Rianimazione, quelle di Ortopedia, con chiare conseguenze sulle attività chirurgiche, e di Pronto soccorso. Una delle regioni più colpite è il Veneto, dove la carenza di personale e di specialisti disponibili a lavorare negli ospedali è tale da produrre un ulteriore problema: a fronte della necessità ufficialmente riconosciuta di 1295 medici specialisti, nei concorsi indetti per la selezione a tempo indeterminato si sta presentando un numero di candidati inferiore a quello richiesto. La stessa regione denuncia infatti che 357 posizioni vacanti non sono state coperte. I numeri più elevati riguardano ancora una volta le specialità legate ad emergenza ed urgenza, anestesia e rianimazione e medicina d'urgenza su tutte, seguite da ginecologia, pediatria, radiologia e ortopedia.

\section{L'emorragia dei pensionamenti}

La carenza di personale medico nelle corsie ospedaliere sarà molto alta nei prossimi anni per il superamento dello scalone previdenziale introdotto dalla riforma Fornero e rischia di subire un'ulteriore brusca accelerazione nel caso in cui vengano inseriti, nella legge di Bilancio in discussione in questi giorni, provvedimenti miranti al suo superamento.

Attualmente escono dal sistema i nati nel 1952 e 1953 con una età media di 65 anni. La curva dei pensionamenti raggiungerà il suo culmine tra il 2018 e il 2022 con uscite per quiescenza valutabili tra $6000 \mathrm{e}$ 7000 ogni anno. Dallo studio della curva demografica (Figura 1) si evince come l'emorragia di medici raggiungerà la cifra di circa 52.000 unità entro il 2025.

È chiaro quindi che non basteranno i giovani medici a sostituire i quiescenti, per colpa dell'errata programmazione degli specialisti perpetrata negli anni passati, ma soprattutto crollerà la qualità generale del 
sistema perché la velocità dei processi in atto non concederà il tempo necessario per il trasferimento di conoscenze dai medici più anziani a quelli con meno esperienza sulle spalle. Si tratta infatti di conoscenze pratiche e capacità tecniche che richiedono tempo e un periodo di passaggio di consegne ed esperienza tra diverse generazioni professionali per essere trasferite correttamente.

\section{Stima della carenza di specialisti per singola specialità. Proiezione al 2025}

Ma quanto impatterà quest'esodo di medici ospedalieri, legato al pensionamento ma anche ad uscite precoci verso il privato, sulle diverse specialità? Abbiamo calcolato una stima, incrociando una proiezione del numero di specialisti uscenti dalle scuole universitarie nei prossimi otto anni, con una previsione dei possibili pensionamenti di specialisti attivi nel SSN al 2025. Abbiamo stimato che solo il $75 \%$ degli specialisti formati scelga di lavorare per il SSN (fonte: rapporto FIASO 2018).

Proiettando al 2025 il numero di specialisti uscenti dalle scuole MIUR previste per il 2018 ( $\mathrm{n}=6934)$, considerato il numero totale di medici specialisti attivi presenti nella rete assistenziale nazionale $(\mathrm{n}=105.310)$ e stimando i pensionamenti dal 2018 al 2025 in 52.700 , il risultato sarebbe un ammanco di circa 16.700 medici.

Le osservazioni sopra esposte, consentono di mettere a confronto, per le principali Specialità, i flussi pensionistici nel SSN nei prossimi 8 anni (fonte: CAT 2016), con le capacità formative post laurea (contratti di formazione specialistica MIUR 2018, ad invarianza di programmazione) nello stesso periodo di riferimento (Figura 1 e Tabella 1). Da questa analisi vediamo che la gran parte delle specialità analizzate andranno in deficit di specialisti, rischiando di impoverire la qualità dei servizi offerti dal SSN, ma per alcune specialità la carenza rispetto al numero di Specialisti formati, sarà maggiore, andando a costituire una vera e propria emergenza, già nel breve termine insostenibile.

In Figura 2 abbiamo indicato le dieci specialità mediche più carenti a livello nazionale nel 2025 secondo le nostre proiezioni. Il dato è stato ottenuto incrociando la stima di nuovi specialisti 2018-2025 entrati nelle scuole di specializzazione negli anni 2014-2021, ad invarianza di programmazione futura rispetto ai posti disponibili nell'anno accademico 2017/18 con le uscite previste nello stesso periodo. Abbiamo incluso nel numero di pediatri mancante anche i pediatri di libera scelta (Fonte dei dati sugli specialisti e sui PLS: CAT 2016).

Va comunque sottolineato come le conseguenze delle stime da noi riportate siano da considerare con severità. Infatti gli organici dei reparti, negli anni precedenti l'anno 2018 considerato per il nostro censimento, hanno sofferto il mancato turnover conseguente al vincolo nazionale della spesa per il personale a partire dal 2010.

\section{Le soluzioni in esame nella legge di bilancio}

L'Anaao ha molto sollecitato l'attuale Governo a porre dei correttivi per far fronte a questa emergenza, stimolando un vivace dibattito.

Ad ora, è stato inserito nelle Legge di Bilancio

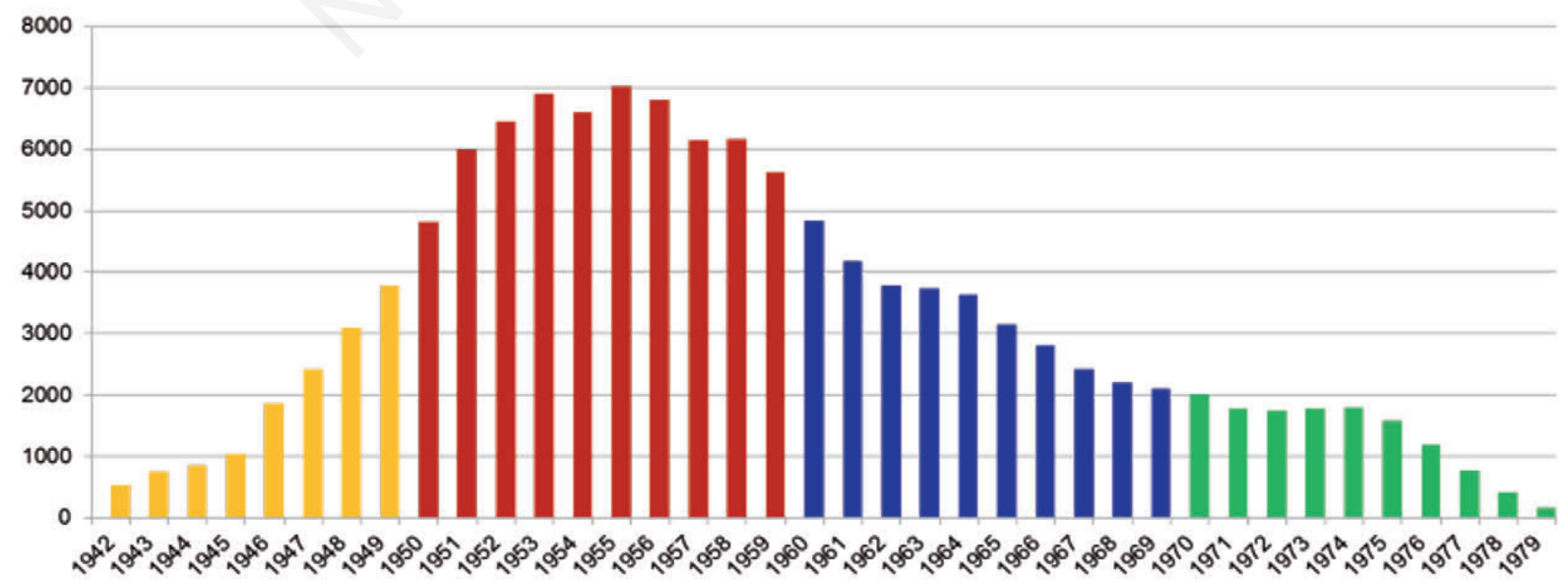

Figura 1. 118.000 medici dipendenti del SSN nel 2008, distribuiti per anno di nascita. Previsione del numero di pensionamenti dal 2018 al 2025 dei Dirigenti Medici del SSN stimata con differenti età di pensionamento. (Enrico Reginato, Carlo Palermo; Sole 24 Ore Sanità: $\left.n^{\circ} 36,2011\right)$. 
per il 2019, l'emendamento che prevede la partecipazione degli Specializzandi dell'ultimo anno a concorsi per Specialisti. L'iniziativa è condivisibile, in quanto permetterebbe di anticipare l'entrata nel mondo del lavoro e di rendere più rapido il già macchinoso sistema concorsuale, necessario per garantire il turnover nei reparti ospedalieri. Poiché infatti gli organici son carenti, anche in molti settori nevralgici degli ospedali, ogni ritardo di sostituzione dei medici andati in pensione determina carichi di lavoro difficili da sostenere per le équipe mediche coinvolte e un conseguente aumento del rischio clinico per operatori e pazienti. Sarebbe comunque auspicabile una previsione più esplicita di assunzione, anche a tempo determinato, degli specializzandi anche prima del conseguimento del titolo. Ma soprattutto, attendiamo una decisa svolta nelle politiche assunzionali eliminando l'anacronistico blocco introdotto con la Finan- ziaria 2010 e incrementando il numero dei contratti di formazione portandoli ad almeno 9500 per avere un congruo numero di specialisti entro il 2025.

\section{Conclusioni}

Le condizioni di lavoro nei reparti ospedalieri e nei servizi territoriali stanno rapidamente degradando. Il blocco del turnover introdotto con la Finanziaria del 2009 ha determinato, ad oggi, una carenza nelle dotazioni organiche di circa 10 mila medici. I piani di lavoro, i turni di guardia e di reperibilità vengono coperti con crescenti difficoltà e una volta occupate le varie caselle si incrocia le dita sperando che nessuno si ammali buttando all'aria il complicato puzzle che bisogna comporre ogni mese. Quindici milioni di ore di straordinario non pagate, numero di turni notturni

Tabella 1. Specialità principali del SSN: pensionamenti dei medici specialisti operanti nel SSN confrontati con i contratti di formazione MIUR ad invarianza di programmazione (proiezione a sette anni, 2018 $\div 2025$ ).

\begin{tabular}{|c|c|c|c|c|c|c|}
\hline Specializzazioni & $\begin{array}{c}\text { Numero di } \\
\text { Specialisti } \\
\text { formati } \\
\text { dal } 2018 \text { al } 2025^{*}\end{array}$ & $\begin{array}{c}\text { Numero di } \\
\text { Nuovi } \\
\text { Specialisti SSN } \\
\text { * 2018-2025** }\end{array}$ & $\begin{array}{l}\text { Specialisti } \\
\text { attivi SSN } \\
\text { anno } 2016 \\
\text { (CAT 2016) }\end{array}$ & $\begin{array}{c}\text { Stima } \\
\text { pensionamenti } \\
2018-2025 * * *\end{array}$ & $\begin{array}{c}\text { Stima } \\
\text { ammanco } \\
\text { Specialisti } \\
\text { al } 2025\end{array}$ & $\begin{array}{c}\% \text { di ammanco } \\
\text { al } 25 \%\end{array}$ \\
\hline Anatomia Patologica & 621 & 466 & 1323 & 673 & -208 & $15,7 \%$ \\
\hline Anestesia Rianimazione e Terapia Intensiva & 5701 & 4276 & 11145 & 5671 & -1395 & $12,5 \%$ \\
\hline Cardiochirurgia & 398 & 299 & 732 & 372 & -74 & $10,1 \%$ \\
\hline Chirurgia Generale & 2904 & 2178 & 6785 & 3452 & -1274 & $18,8 \%$ \\
\hline Chirurgia Pediatrica & 123 & 92 & 349 & 178 & -86 & $24,5 \%$ \\
\hline Ematologia & 759 & 569 & 1396 & 710 & -141 & $10,1 \%$ \\
\hline Farmacologia e Tossicologia Clinica & 184 & 138 & 533 & 271 & -133 & $25,0 \%$ \\
\hline Ginecologia e Ostetricia & 2437 & 1828 & 4858 & 2472 & -644 & $13,3 \%$ \\
\hline Igiene e Medicina Preventiva & 1448 & 1086 & 2876 & 1463 & -377 & $13,1 \%$ \\
\hline Malattie dell'Apparato Cardiovascolare & 2606 & 1954 & 5234 & 2663 & -709 & $13,5 \%$ \\
\hline Malattie dell’Apparato Respiratorio & 805 & 603 & 1442 & 734 & -130 & $9,0 \%$ \\
\hline Malattie Infettive e tropicali & 713 & 535 & 1342 & 683 & -148 & $11,1 \%$ \\
\hline Medicina d'Emergenza-Urgenza & 1962 & 1471 & 11107 & 5652 & -4180 & $37,6 \%$ \\
\hline Medicina Interna & 2705 & 2029 & 7580 & 3857 & -1828 & $24,1 \%$ \\
\hline Microbiologia e Virologia & 130 & 98 & 338 & 172 & -74 & $22,0 \%$ \\
\hline Nefrologia & 989 & 741 & 2119 & 1078 & -337 & $15,9 \%$ \\
\hline Neurochirurgia & 498 & 374 & 850 & 433 & -59 & $6,9 \%$ \\
\hline Neurologia & 1295 & 971 & 2281 & 1161 & -189 & $8,3 \%$ \\
\hline Oftalmologia & 1004 & 753 & 1816 & 924 & -171 & $9,4 \%$ \\
\hline Ortopedia e Traumatologia & 2100 & 1575 & 4055 & 2063 & -489 & $12,0 \%$ \\
\hline Otorinolaringoiatria & 897 & 672 & 1707 & 869 & -196 & $11,5 \%$ \\
\hline Patologia Clinica e Biochimica clinica & 575 & 431 & 1551 & 789 & -358 & $23,1 \%$ \\
\hline Pediatria & 3740 & 2805 & 12042 & 6127 & -3323 & $75,3 \%$ \\
\hline Psichiatria & 1954 & 1466 & 4712 & 2398 & -932 & $19,8 \%$ \\
\hline Radiodiagnostica & 3311 & 2483 & 6067 & 3087 & -604 & $10,0 \%$ \\
\hline Statistica Sanitaria & 15 & 11 & 386 & 196 & -185 & $47,9 \%$ \\
\hline Urologia & 966 & 724 & 1786 & 909 & -185 & $10,3 \%$ \\
\hline
\end{tabular}

*calcolo su stima numero di borse perse specialità 2014-2021; **calcolo su stima ingresso specialisti nel SSN del $75 \%$ sul totale; ***calcolo dei pensionamenti secondo riforma pensionistica "QUOTA 100". 


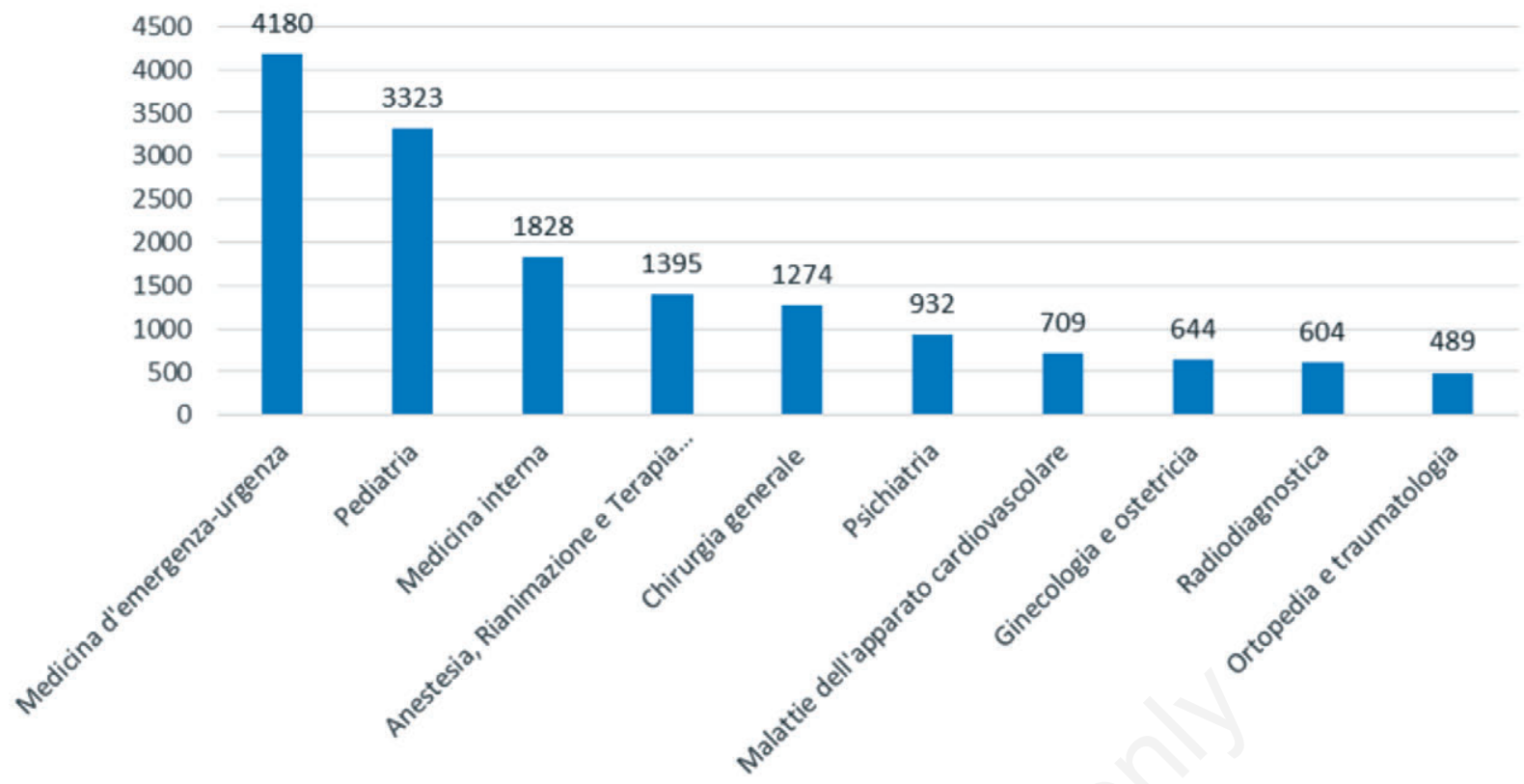

Figura 2. Le dieci specialità mediche più carenti a livello nazionale nel 2025 in caso di approvazione della proposta di riforma della legge Fornero con pensionamento anticipato a 62 anni con almeno 38 anni di contributi. Il numero di pediatri mancante include anche i pediatri di libera scelta. (Fonti dei dati sugli specialisti e sui PLS: CAT 2016, Ministero Salute 2010).

e festivi pro-capite in crescita, fine settimana quasi sempre occupati tra guardie e reperibilità, difficoltà a poter godere perfino delle ferie maturate rappresentano gli elementi su cui si fonda oggi la sostenibilità organizzativa ed economica degli ospedali italiani.

Regioni e Aziende dal 2009 a oggi hanno risparmiato tagliando sul personale, il Bancomat che è stato ferocemente sfruttato per raggiungere l'equilibrio di bilancio. Non si tratta solo di turnover ma anche di gravidanze o di assenze per malattie prolungate mai sostituite. Il risparmio per le aziende relativamente ai soli medici e dirigenti sanitari per il 2018 è valutabile intorno al miliardo di euro. Ormai la situazione è pesante ed i numeri del presente lavoro indicano che la prospettiva rischia di avvitarsi verso il dramma, arrivando addirittura alla mancanza di specialisti pur in presenza di uno sblocco del turnover, in mancanza di interventi che determinino rapidamente un cambiamento. Le soluzioni sono state indicate in due punti qualificanti del Contratto di Governo. Si afferma, infatti, che Il problema dei tempi di attesa è susseguente anche alla diffusa carenza di medici specialisti, infermieri e personale sanitario. Ė dunque indispensabile assumere il personale medico e sanitario necessario, anche per dare attuazione all'articolo 14 della legge n. 161/2014. Ed ancora si ribadisce che I posti per la formazione specialistica dei medici dovrebbero essere determinati dalle reali necessità assistenziali e tenendo conto anche dei pensionamenti, assicurando quindi un'armonizzazione tra posti nei corsi di laurea e posti nel corso di specializzazione.

È necessario, pertanto, non solo sbloccare il turnover ma incrementare anche il finanziamento per le assunzione ed attivare i miliardi di risparmi effettuati dalle Regioni nell'ultimo decennio. Per quanto attiene la formazione post laurea, è arrivato il momento di una riforma globale passando ad un contratto di formazione/lavoro e alla valorizzazione di una rete di ospedali di insegnamento. L'attuale sistema formativo, in particolare nella parte specialistica post-lauream, se confrontato con quello degli altri Paesi Europei, appare obsoleto ed espressione di un arroccamento dell'Università che, pur di non perderne l'egemonia, è disposta a barattare la qualità formativa e la performance dell'intera programmazione di medici specialisti.

Si è giunti oggi ad un bivio ineluttabile: o si cambia direzione, o la sopravvivenza del SSN è in serio pericolo. 


\title{
I rischi di un eccesso di cure negli anziani: fare di più non significa fare meglio
}

\author{
Marco Bobbio \\ già direttore Struttura complessa di Cardiologia, Ospedale Santa Croce e Carle, Cuneo; Segretario Generale di Slow Medicine, \\ Italia
}

\section{Introduzione}

Abbiamo a disposizione un numero straordinario di farmaci e di procedure diagnostiche in grado di curare e guarire malattie una volta invalidanti e mortali, ma corriamo anche il rischio di indurre danni e sofferenze, quando prescriviamo test e trattamenti in modo inappropriato o del tutto incongruo, a persone che a fronte di benefici prevedibili irrisori ne subiranno le conseguenze. ${ }^{1}$

Due aspetti vanno evidenziati: il numero delle persone politrattate aumenta negli anni; nel biennio 199920001 ' $8,2 \%$ di pazienti assumeva 5 o più farmaci diversi e dieci anni dopo la percentuale era aumenta al $15 \%$ (differenza $6,6 \%$; intervallo di confidenza al $95 \%$, da $4,4 \%$ a $8,2 \%)^{2}$ in secondo luogo il problema è ulteriormente accentato nelle persone anziane in quanto la presenza di comorbidità, legata all'età, aumenta la probabilità di una poliprescrizione. Dal Rapporto Nazionale 2017 sull'Uso dei Farmaci in Italia dell'Osservatorio sull'Impiego dei Medicinali (OsMed) dell' $\mathrm{AIFA}^{3}$ si ricava che la popolazione con più di 64 anni assorbe oltre il $60 \%$ della spesa in assistenza convenzionata. Il $94 \%$ degli anziani nel corso del 2017 ha ricevuto almeno una prescrizione; complessivamente i soggetti tra 65 e 69 anni hanno assunto 7,7 farmaci diversi (il 21,6\% dei soggetti ha assunto più di 10 principi attivi diversi) e quelli con età pari $\mathrm{o}$ superiore a 85 anni 11,8. Per quanto riguarda la prevalenza d'uso dei farmaci in età geriatrica si sono collocati al primo posto i medicinali antiulcera peptica e malattia da reflusso gastroesofageo $(48,3 \%)$, seguiti

Corrispondente: Marco Bobbio, via Pietra del Gallo 45, 10025 Pino Torinese (TO), Italia.

E-mail: mbobbio51@gmail.com

Articolo pubblicato secondo la Creative Commons Attribution NonCommercial 4.0 License (CC BY-NC 4.0).

CC Copyright M. Bobbio, 2019

Licensee PAGEPress, Italy

QUADERNI - Italian Journal of Medicine 2019; 7(4):26-30 dagli antitrombotici (41,5\%), dalle sostanze modificatrici dei lipidi $(34,4 \%)$ e dai farmaci antinfiammatori ed antireumatici non steroidei $(32,8 \%)$.

\section{Cause della politerapia}

In molti casi la politerapia è giustificata da condizioni cliniche che richiedono necessariamente la prescrizione di più farmaci, ma è stato anche dimostrato che 1 su 5 farmaci comunemente usati nelle persone anziane viene prescritto in modo inappropriato 4 e che tale percentuale aumenta a 1 su 3 nelle persone ricoverate in case di riposo. ${ }^{5} \mathrm{Da}$ una analisi sistematica della letteratura, mettendo insieme i dati ricavati da 44 ricerche, è stata osservata un'associazione positiva della politerapia con il numero di medici prescrittori, con la presenza di malattie metaboliche e di disfunzioni neurologiche; è stata invece riscontrata un'associazione tra il basso numero di farmaci prescritti con l'età avanzata, con la presenza di difetti cognitivi e di disabilità nella vita di tutti i giorni e con la durata del ricovero un casi di cura. ${ }^{6}$ Uno dei meccanismi che inducono la politerapia inappropriata è la cosiddetta $c a$ scata terapeutica, il meccanismo per cui un effetto avverso di un farmaco viene erroneamente diagnosticato come un sintomo di un'altra patologia e trattato con un'ulteriore farmaco invece che con la riduzione del dosaggio o con la sostituzione del farmaco che ha provocato il nuovo sintomo. ${ }^{7}$ Spesso i clinici, quando devono affrontare un sintomo, si focalizzando più sugli ipotetici benefici di un trattamento per la patologia di loro competenza e che sui possibili rischi da interazioni con farmaci già in uso ${ }^{8}$ (Tabella 1 ).

Per evitare di prescrivere inutilmente un nuovo farmaco il medico dovrebbe porsi queste domande: i) Il nuovo sintomo può dipendere da un farmaco che il paziente sta assumendo? ii) Il farmaco che ha provocato il sintomo è essenziale? Quali sono i rischi e i benefici di continuare quel farmaco?

D'altra parte anche il paziente dovrebbe chiedere al medico: invece di aggiungere una nuova medicina, cosa mi può succedere se sospendiamo o sostituiamo quella che mi ha provocato i disturbi? 


\section{Choosing Wisely}

Essendo stati osservati i danni provocati da un eccesso di prescrizioni, in tutto il mondo sono stati avviati progetti per evitare le prescrizione a rischio di inappropriatezza e per ridurre le prescrizioni inappropriate. Per evitare le prescrizioni inutili è stato lanciato il programma Choosing Wisley. Nel 2010 Howard Brody ${ }^{9}$ propose, per arginare la spinta dei sistemi sanitari e delle assicurazioni a razionare le prestazioni sanitarie, che le società scientifiche definissero una lista di cinque test diagnostici, trattamenti medici o chirurgici comunemente prescritti che, secondo le conoscenze scientifiche disponibili, non apportano benefici significativi ai pazienti e li espongono a inutili rischi. Nel 2012 la Fondazione ABIM (American Board of Internal Medicine), con la collaborazione di Consumer Reports, un'organizzazione di consumatori non profit e indipendente, aderì alla proposta di Brody e lanciò l'iniziativa Choosing Wisely, ${ }^{10}$ invitando le società scientifiche ad individuare una lista di 5 test $\mathrm{o}$ trattamenti (the top 5 list), comunemente utilizzati nella propria specialità, il cui impiego possa diventare oggetto di confronto tra medici e pazienti. Nell'aprile del 2012, nove società scientifiche pubblicarono le prime liste, seguite nei mesi successivi da molte altre. Quattro anni dopo le società scientifiche sono diventate più di 70 e hanno individuato più di 400 test e trattamenti sanitari. Un aspetto qualificante del progetto è che le raccomandazioni non rappresentano solo un richiamo ai professionisti, ma dovrebbero diventare uno strumento per avviare un dialogo onesto tra medico e paziente, per facilitare scelte informate e condivise. Negli anni successivi, l'iniziativa si è estesa in molti altri stati; attualmente sono attivi progetti in $\mathrm{Au}-$ stria, Brasile, Canada, Corea del Sud, Danimarca, Francia, Galles, Germania, Giappone, India, Inghilterra, Israele, Nuova Zelanda, Olanda, Svizzera.

Già nel 2012 in Italia il progetto venne lanciato e coordinato da Slow Medicine, un' associazione di professionisti della sanità, pazienti e cittadini che si batte per una medicina sobria, rispettosa e giusta, ${ }^{11}$ con il titolo Fare di più non significa fare meglio - Choosing
Wisely Italy. Anche da noi si è creata l'alleanza con un'associazione di consumatori (Altroconsumo) che ha preparato delle schede informative per i cittadini e i pazienti nelle quali vengono spiegati in modo semplice i rischi di un uso eccessivo di test e trattamenti. ${ }^{12}$ In questo modo viene attribuito ai professionisti della salute in alleanza con pazienti e cittadini la responsabilità di assumere iniziative capaci di promuovere l'uso appropriato delle risorse disponibili. ${ }^{13,14}$ All'inizio 2019 in Italia avevano aderito al progetto 44 Società scientifiche di medici, farmacisti, fisioterapisti, infermieri (tra cui la FADOI) e erano state definite 220 raccomandazioni su pratiche a rischio d'inappropriatezza. ${ }^{15}$ Negli Stati Uniti l'American Geriatric Society ha individuato 10 raccomandazioni a rischio di inappropriatezza e altre società hanno proposto raccomandazioni specifiche per i soggetti anziani (Tabella 2). Anche in Italia alcune raccomandazioni di varie società sono rivolte a prestare attenzione ai pazienti anziani (Tabella 2). Da febbraio 2019 è stata messa a disposizione di medici e pazienti un'App, scaricabile gratuitamente su smartphone con sistema Android e iOS, per trovare rapidamente le raccomandazioni segnalate dalle società scientifiche e per accedere alla schede prodotte da Altroconsumo.

\section{La deprescrizione}

Per ridurre il numero di farmaci prescritti in modo inappropriato o diventati inutili nel tempo sono in corso vari progetti per diffondere il concetto che in determinate condizioni eliminare uno o più farmaci da una lista complessa può essere un vantaggio in termini di salute e di benessere, senza far correre rischi al paziente. La Canadian Deprescribing Network ${ }^{16}$ svolge un compito informativo per far crescere la consapevolezza sulla sicurezza dei medicinali, sulla deprescrizione e sulle alternative più sicure all'uso di farmaci. $\mathrm{Nel}$ sito si trova materiale dedicato ai pazienti. ${ }^{17}$ Anche in Italia gruppi di medici di medicina generale e organizzazioni sanitarie a Bologna, Modena, Novara e Torino hanno avviato alcuni progetti per ridurre le prescrizioni. Il termine deprescrizione significa elimi-

Tabella 1. Effetti negativi della politerapia.

Riduzione dell'efficacia di uno o più farmaci

Aumento del rischio di effetti avversi attuali o potenziali

Aumento del rischio di interazione con uno o più farmaci

Aumento del rischio di irregolarità nell'assunzione delle medicine

Aumento del rischio di sospensione spontanea di uno o più farmaci

Sviluppo di controindicazioni

Aumento del rischio di sintomi geriatrici (cadute, delirium, declino cognitivo, malnutrizione) 
nare terapia a lungo termine, sotto stretto controllo medico e nella letteratura scientifica viene preferito ai termini cessazione, sospensione o interruzione che non contemplano il ruolo attivo del medico nel processo decisionale; in altre parole il medico deve adottare la stessa metodologia clinica che usa per prescrivere un farmaco anche per deprescriverlo, dal momento che non si tratta solo di un meccanismo di mera sospensione. Gli autori anglosassoni sottolineano il fatto che comunque il termine deprescrizione è una parola coraggiosa perché l'aspettativa dei pazienti e dei parenti è sempre quella di aver tutto il possibile, senza preoccuparsi delle interazioni tra farmaci. ${ }^{18,19}$ In Italia invece alcuni esperti sostengono che il termine deprescrizione possa spaventare i pazienti con l'idea che si voglia risparmiare risorse economiche sulla loro salute. Il Ministero della Salute ha adottato il termine riconciliazione della terapia farmacologica per indicare un processo formale che permette, in modo chiaro e completo, di rilevare e conoscere la terapia farmacologica seguita assieme ad altre informazioni relative al paziente e consente al medico prescrittore di valutare con attenzione se proseguirla, variarla o interromperla del tutto o in parte. Con la deprescrizione - ricorda la nota - vengono esclusi dalle terapie i farmaci ritenuti a vario titolo non più necessari; la riconciliazione terapeutica, invece, si pone l'obiettivo di verificare quanto il paziente segue le raccomandazioni del medico su dosi, tempi e frequenza di assunzione e migliorare il livello di aderenza alla terapia. Altri autori infine preferiscono il termine armonizzazione terapeutica delle cure.

In realtà la deprescrizione è un processo delicato che va adottato con le dovute cautele perché preoccupa i pazienti, può provocare la ricomparsa di sintomi e deve essere sempre concordato con il paziente che svolge un ruolo fondamentale nel condividere il processo. ${ }^{20}$ Nella letteratura sono stati individuate numerose difficoltà alla deprescrizione che vanno conosciute per saperle affrontare (Tabella 3).

Recentemente sta aumentando il numero di articoli che descrivono protocolli utilizzati per adottare una deprescrizione sicura e che hanno portato a una riduzione importante del numero di farmaci, senza aver creato effetti indesiderati, con una piena soddisfazione da parte dei pazienti che avevano un minor carico di farmaci da assumere ogni giorno..$^{21-24}$

\section{INTERCheck}

L'Istituto Farmacologico Mario Negri ha messo a punto un programma INTERCheck ${ }^{25,26}$ che permette di migliorare l'appropriatezza prescrittiva nel paziente anziano, attraverso un approccio di valutazione delle terapie che tiene in considerazione diversi aspetti della farmacologia geriatrica tra cui le interazioni tra farmaci, i farmaci potenzialmente inappropriati nell'anziano secondo differenti criteri delle letteratura scientifica (Beers; START/STOPP) e consiglia come sospendere i farmaci che necessitano riduzione graduale delle dosi, come modificare il dosaggio in soggetti con alterata funzionalità renale, come identificare pazienti a maggior rischio di effetti indesiderati con l'algoritmo GerontoNet ADR Risk Score. ${ }^{27}$ Il programma e l'App possono essere scaricati gratuita-

\title{
Tabella 2. Raccomandazioni di Choosing Wisely riguardanti i pazienti anziani.
}

\begin{abstract}
Stati Uniti
Ha aderito alla campagna Choosing Wisely l'American Geriatric Society che ha individuato come pratiche a rischio di inappropriatezza le seguenti 10: non utilizzare l'alimentazione parenterale nei pazienti con demenza; non usare gli antipsicotici come primo approccio nel trattamento dei pazienti con sintomi di demenza; evitare l'uso di altri farmaci oltre la metformina per raggiungere $\mathrm{HgA1}$ c $<7,5$; non usare benzodiazepine come prima scelta nel caso di insonnia, agitazione e delirium; non usare antimicrobici per trattare batteriurie in soggetti asintomatici; non prescrivere inibitori della colinesterasi per la memoria senza periodiche valutazioni dei benefici cognitivi; non raccomandare gli screening per il tumore della mammella, del colonretto, della prostata e dei polmoni senza tener conto dell'aspettativa di vita; evitare l'uso di stimolanti dell'appetito per trattare l'anoressia e la cachessia negli anziani; non prescrivere un nuovo farmaco senza aver rivisto la lista di quelli che il paziente sta già assumendo; non usare costrizioni fisiche per controllare disturbi comportamentali nei pazienti ospedalizzati con delirium. Hanno fornito raccomandazioni che riguardano gli anziani anche l'American Academy of Nursing (non lasciare a lungo a letto o in poltrona i pazienti anziani ricoverati; non usare costrizioni fisiche per controllare disturbi comportamentali nei pazienti ospedalizzati con delirium) e l'American Pshyciatric Association (non usare gli antipsicotici come primo approccio nel trattamento dei pazienti con sintomi di demenza; non prescrivere di routine antipsicotici come primo approccio per l'insonnia)

\section{Italia}

La Federazione Nazionale degli Ordini delle Professioni Infermieristiche (FNPOI) raccomanda di non utilizzare disinfettanti sitolesivi sulla cute integra nei soggetti anziani allettati con cute fragile e/o compromessa, la Cochrane Neurological Sciences Field (CNF) di non usare benzodiazepine o altri ipnotici negli anziani come prima scelta nell'insonnia, la Federazione delle Associazioni dei Dirigenti Ospedalieri Internisti (FADOI) di non trattare con antibiotici la batteriuria nei soggetti anziani in assenza di sintomi urinari, di non usare benzodiazepine negli anziani come prima scelta per insonnia, agitazione e delirium, di non prescrivere di routine farmaci ipolipemizzanti nei pazienti con aspettativa di vita limitata, la Società Italiana di Farmacia Clinica e Terapia (SIFACT) di non prescrivere il vaccino antinfluenzale MF59 nella popolazione anziana e infine la Società Italiana di Medicina Generale (SIMG) di non prescrivere di routine in prima istanza benzodiazepine o Z-drugs nei pazienti anziani in caso di insonnia.
\end{abstract}


Tabella 3. Barriere alla deprescrizione.

Difficoltà di interazione tra medico e paziente

Tempi ridotti per le visite

Frammentazione delle decisioni terapeutiche tra vari professionisti

Incomplete informazioni sui motivi che avevano giustificato un trattamento

Non conoscenza delle intolleranze ai farmaci

Cambiamento nel tempo degli obiettivi terapeutici

Incertezza sui benefici e sui rischi di continuare o interrompere un farmaco specifico

Abitudini prescrittive consolidate

Preoccupazioni per gli effetti avversi dovuti alla sospensione

Pressione alla prescrizione indotta da raccomandazioni contenute in specifiche linee guida

Medicina difensiva

mente e sono un valido supporto per le decisioni cliniche al letto del malato, quando si scrive la lettera di dimissione e in ambulatorio. Alcuni reparti di medicina interna e geriatria l'hanno già adottato e lo utilizzano routinariamente.

\section{Conclusioni}

L'eccessiva prescrizione di procedure diagnostiche e di trattamenti è un problema crescente e sempre più evidenziato nella letteratura scientifica per cui sono stati avviati programmi per evitare le prescrizione a rischio di inappropriatezza (Choosing Wisely) e per ridurre le prescrizioni inappropriate (deprescrizione).

È necessario avviare parallelamente corsi di aggiornamento per una prescrizione accorta che si devono adeguare ai seguenti principi: ${ }^{28}$ i) non prendere in considerazione solo il trattamento farmacologico; ii) adottare diverse strategie prescrittive; iii) prestare la massima attenzione agli effetti avversi; iv) adottare con cautela e scetticismo i nuovi farmaci; v) coinvolgere il paziente su un programma condiviso; vi) prendere in considerazione gli effetti a lungo termine; vii) spiegare al paziente il motivo di una prescrizione; viii) valutare se qualche farmaco può essere sospeso.

Medici e pazienti devono condividere la responsabilità dell'inappropriatezza. Sottolineare che le ripercussioni associate con un cattivo uso delle terapia si può tradurre in un aumento della soddisfazione del paziente e a un miglioramento della cura. Penso che sia necessario far crescere un salubre scetticismo sul valore e sull'efficacia delle terapie, per evitare l'induzione di una futile politerpia che può mettere a rischio la salute del paziente.

\section{Messaggi chiave}

- Il numero delle persone politrattate aumenta negli anni.

- Nel corso del 2017 in Italia i soggetti tra 65 e 69 anni hanno assunto in media 7,7 farmaci diversi e i soggetti di età pari o superiore a 85 anni 11,8 .

- 1 su 5 farmaci comunemente usati nelle persone anziane viene prescritto in modo inappropriato.

- Uno dei meccanismi che inducono la politerapia inappropriata è la cascata terapeutica, il meccanismo per cui un effetto avverso di un farmaco viene erroneamente diagnosticato come un sintomo di un'altra patologia e trattato con un'ulteriore farmaco.

- Il progetto internazionale Choosing Wisely ha invitato le società scientifiche ad individuare una lista di 5 test o trattamenti comunemente utilizzati nella propria specialità, il cui impiego possa diventare oggetto di confronto tra medici e pazienti.

- In Italia Slow Medicine ha lanciato e coordina il progetto Fare di più non significa fare meglio Choosing Wisely Italy.

- Con il termine deprescrizione si intende la valutazione della terapia a lungo termine, sotto stretto controllo medico, per individuare quali farmaci non sono più indispensabili.

- Sono disponibile le App di Choosing Wisely e di INTERCheck per aiutare i medici a una individuare in modo appropriato i farmaci da prescrivere o eventualmente sospendere.

\section{Bibliografia}

1. Bobbio M, Lusiani L, Frediani R. Troppe cure. Quaderni Ital J Med 2014;2:131-4.

2. Kantor ED, Rehm CD, Haas JS, et al. Trends in prescription drugs use among adults in the United States from 1999-2012. JAMA 2015;314:1881-21.

3. Osservatorio Nazionale sull'impiego dei Medicinali. L'uso dei farmaci in Italia. Rapporto Nazionale 2017. Roma: Agenzia Italiana del Farmaco; 2018. Disponibile a: http://www.aifa.gov.it/sites/default/files/Rapporto_Os Med_2017_AIFA.pdf

4. Roughead EE. Potentially inappropriate prescribing among Australian veterans and war widows/widowers. Intern Med J 2007;37:402. 
5. Stafford AC. Inappropriate prescribing in older residents of Australian care homes. J Clin Pharm Ther 2011; 36:33.

6. Jokanovic N, Tan ECK, Dooley MJ, et al. Prevalence and factors associated with polypharmacy in long-term care facilities: A systematic review. JAMDA 2015;16: 535,e1-e12.

7. Rochon PA, Gurwitz JH. The prescribing cascade revisited. Lancet 2017;389:1778-80.

8. Bobbio M, Lusiani L, Frediani R. Overtreatment. Ital J Med 2015;9:204-6.

9. Brody H. Medicine's ethical responsibility for health care reform: the Top Five list. N Engl J Med 2010; 362:283-5.

10. Cassel CK, Guest JA. Choosing wisely: helping physicians and patients make smart decisions about their care. JAMA 2012;307:1801-2.

11. Sito ufficiale di Slow Medicine: https://www.slowmedicine.it

12. Altroconsumo. Cure in eccesso: un rischio per la salute; 08 ottobre 2018. Disponibile a: https://www.altroconsumo.it/salute/dal-medico/speciali/esami-inutili

13. Domenighetti G, Vernero S. Looking for waste and inappropriateness: if not now, when? Intern Emerg Med 2014;9:S1-7.

14. Vernero S, Domenighetti G, Bonaldi A. Italy's "Doing more does not mean doing better" campaign. BMJ 2014;349:g4703.

15. Sito ufficiale di Choosing Wisely Italy: http://www.choosingwiselyitaly.org/

16. Sito ufficiale del Canadian Deprescribing Network: https://www.deprescribingnetwork.ca/

17. Tannenbaum C. You may be at risk; 2014. Available from: http:/www.criugm.qc.ca/fichier/pdf/BENZOeng.pdf

18. Reeve E, Turner JP. Patients' perspectives on the brave new word 'deprescribing'. Int J Pharm Pract 2015; 23:90-1.

19. Alldred DP. Deprescribing: a brave new word? Int J Pharm Pract 2014;22:2-3.

20. Lown Institute. Deprescribing starts with shared decision making. Available from: https://lowninstitute.org/news/ blog/deprescribing-starts-shared-decision-making/

21. Reeve E, Shakib S, Hendrix I, et al. Review of deprescribing processes and development of an evidencebased, patient-centred deprescribing process $\mathrm{Br} \mathrm{J}$ Clin Pharmacol 2014;78:738-47.

22. Avery AJ, Bell BG. Rationalising medications through deprescribing. BMJ 2019;364:1570.

23. Parekh N, Schenker Y, Good CB, Neilson L, Shrank WH. Deprescribing in advanced illness: Aligning patient, clinician, and health plan goals. J Gen Intern Med 2019 [Epub ahead of print].

24. Ailabouni N, Mangin D, Nishtala PS. DEFEATpolypharmacy: deprescribing anticholinergic and sedative medicines feasibility trial in residential aged care facilities. Int J Clin Pharm 2019;41:167-78.

25. InterCheck WEB. Strumento per la valutazione dell'appropriatezza prescrittiva. Disponibile a: https://clinicalweb.marionegri.it/intercheckweb/

26. Ghibelli S, Marengoni A, Djade C D, et al. Prevention of inappropriate prescribing in hospitalized older patients using a computerized prescription support system (INTERcheck ${ }^{\circledR}$ ). Drugs Aging 2013;30:821-8.

27. Pasina L, Marengoni A, Ghibelli S, et al. A multicomponent intervention to optimize psychotropic drug prescription in elderly nursing home residents: An Italian multicenter, prospective, pilot study. Drugs Aging 2016;33:143-9.

28. Schiff GD, Galater WL, Duhig J et al. Principles of conservative prescribing. Arch Intern Med 2011;171:1433-40. 


\title{
Pensionamento: ritirarsi dal lavoro, non dalla vita né da un futuro di buona salute
}

\author{
Roberto Nardi, ${ }^{1}$ Daniele Borioni, ${ }^{1}$ Daniela Tirotta, ${ }^{2}$ Franco Berti, ${ }^{3}$ Giuliano Pinna,,${ }^{4}$ Shirley Ehrlich, ${ }^{5}$ \\ Giovanna Pentella, ${ }^{6}$ Paola Cosma, ${ }^{7}$ Roberta Rapetti, ${ }^{8}$ Andrea Fontanella ${ }^{6}$
}

${ }^{1}$ Medicina Interna, Bologna; ${ }^{2}$ Medicina Interna, Cattolica (RN); ${ }^{3}$ Medicina Interna, Roma; ${ }^{4}$ Medicina Interna, Asti; ${ }^{5}$ Medicina Generale, Imola (BO); ${ }^{6}$ Medicina Interna, Ospedale del Buon Consiglio - Fatebenefratelli, Napoli; ${ }^{7}$ Cairo Montenotte (SV);

${ }^{8}$ Dipartimento di Medicina, Asl n. 2 Savonese, Savona, Italia

\section{Il contesto epidemiologico-demografico}

Invecchio imparando ogni giorno nuove cose

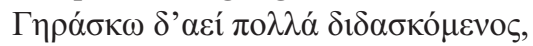
Solone, Elegie

In Italia, secondo i dati ISTAT 2016, gli ultrasessantacinquenni sono 13,4 milioni, il $22 \%$ del totale e nel 2050 saranno oltre i 20 milioni. ${ }^{1}$ In diminuzione risultano sia la popolazione in età attiva di 15-64 anni (39 milioni, il $64,3 \%$ del totale) sia quella fino a 14 anni di età ( 8,3 milioni, il $13,7 \%)$. Dai dati presentati dall'Istituto di Statistica alla Camera, in occasione del suo ventiseiesimo rapporto annuale del maggio 2018, si evince che ogni 100 giovani si contano oggi 170 anziani e che tra dieci anni per 100 giovani in Italia ci saranno ben 217 anziani. L'indice di dipendenza strutturale sale al $55,5 \%$, quello di dipendenza degli anziani al 34,2\%. Diminuisce la speranza di vita alla nascita. Per gli uomini si attesta a 80,1 anni (da 80,3 del 2014), per le donne a 84,7 anni (da 85). L'età media della popolazione aumenta di due decimi e arriva a 44,6 anni. ${ }^{2} \mathrm{Nel}$ periodo 2000-2030 si stima che la popolazione anziana aumenterà dal $15,5 \%$ al $24,3 \%$ in Europa, con punte di ultra 65 enni fino al 33\% nel 2030. La rivoluzione del XXI secolo ha riguardato il cosiddetto longevity shock, che ha comportato un considerevole aumento della speranza di vita (calcolata alla nascita, secondo i dati OMS del 2014, a 80,5 anni negli uomini e a 84,8 nelle donne, con un'aspettativa di vita in buona salute di 72,8 anni) ed

Corrispondente: Roberto Nardi, Medicina Interna, Bologna, Italia.

E-mail: nardidoc48@gmail.doc

Articolo pubblicato secondo la Creative Commons Attribution NonCommercial 4.0 License (CC BY-NC 4.0).

CCopyright R. Nardi et al., 2019

Licensee PAGEPress, Italy

QUADERNI - Italian Journal of Medicine 2019; 7(4):31-47 un cospicuo incremento demografico delle persone che raggiungono un'età molto avanzata (long survivors). ${ }^{3}$ $\mathrm{Si}$ è verificato un cambiamento della nosologia dei pazienti che afferiscono al Servizio Sanitario Nazionale, con prevalenza di malattie cronico-degenerative, alcune delle quali invalidanti. Valutando la speranza di vita per condizioni di salute si evidenzia un calo significativo degli anni vissuti in buona salute (Healthy Life Years Expectancy, HLYE). Secondo i dati dell'European Union Statistics On Income And Living Conditions (EU-SILC) del 2011, all'età di 65 anni, le donne possono aspettarsi di vivere 7,0 anni (31\% della vita rimanente) senza limitazioni nelle attività (misura che coincide con HLYE), 9,4 anni (42\%) con limitazioni moderate nelle attività e 6,1 anni (27\%) con limitazioni gravi nelle attività. Gli uomini della stessa età possono aspettarsi di vivere 8,1 anni ( $43 \%$ della vita rimanente) senza limitazioni di attività, 6,8 anni (36\%) con limitazioni di attività moderate e 3,8 anni $(20 \%)$ con limitazioni di attività gravi. ${ }^{4}$ In Europa le previsioni riportate all'anno 2060 configurano un quadro esplosivo: la quota di popolazione da 0-14 anni è prevista costante, mentre diminuirà quella nella fascia 15-67 anni, passando dal 67 al 56\%. A fronte di ciò gli ultra65enni passeranno dal 18 al $30 \%$ della popolazione: uno su tre. Gli ultra80enni, poi, passeranno dal 5 al 12\%. Si modificherà definitivamente il rapporto fra vecchi e giovani (demographic old-age dependency ratio), ossia il rapporto fra gli ultra65enni e quelli fra 0-64 anni. Si prevede che tale indice passerà dall'attuale $26 \% \mathrm{al} 52,5 \%$, per cui anziché avere quattro persone in età di lavoro per ogni ultra65enne, com'era nel 2010, ne avremo solo due. ${ }^{5}$ Lo scenario che si staglia nel futuro non è roseo: è quello di una popolazione autoctona che diminuisce e invecchia, con un impoverimento soprattutto della componente più giovane e con una prevalenza delle età adulta-anziana al centro della vita lavorativa del paese. ${ }^{6}$ In tale contesto demografico è necessario intraprendere con urgenza una strategia politica congruente, sia per il mercato del lavoro che per gli aspetti sanitari correlati. È utile che i singoli individui che vanno incontro alla 
senescenza siano consapevoli delle implicazioni (economiche e relative alla salute) correlate alla cessazione dell'attività lavorativa, ${ }^{7,8}$ nonché delle relazioni tra lavoro ed invecchiamento. ${ }^{9}$

\section{Le fasi del pensionamento: adattamento e disadattamento}

Il pensionamento non costituisce un evento brusco e subitaneo, ma si svolge attraverso diverse fasi, cui dovrebbe corrispondere un adattamento da parte del singolo individuo (Tabella 1). ${ }^{10}$

Le fasi del pensionamento riproducono quelle descritte, in generale, nel modello psico-dinamico del cambiamento di Elizabeth Kübler-Ross ${ }^{11}$ (Figura 1). ${ }^{12}$

Alla luce delle fasi descritte, emergono alcuni concetti: i) l'elaborazione e l'adattamento al pensionamento sono migliori se esiste una pianificazione precedente, mediata tra attività lavorativa, famiglia $\mathrm{e}$ tempo libero o se intervengono comportamenti proattivi preventivi finalizzati a contrastare il disadattamento; ii) l'interruzione brusca dell'attività lavorativa può in alcuni casi comportare uno shock da pensionamento, particolarmente in alcune condizioni di rischio; iii) un rallentamento della velocità di transizione tra attività lavorativa e pensionamento può facilitare l'adattamento del singolo individuo.

\section{La crisi del ruolo e d'identità dopo la cessazione dell'attività lavorativa}

\author{
Spesso commettiamo l'errore di confondere \\ la nostra vita con il lavoro. \\ Il nostro lavoro non è la nostra vita
}

L'uomo della società industriale e post-industriale

Tabella 1. Le fasi del pensionamento.

\begin{tabular}{ll}
\hline Fase & Caratteristiche \\
\hline Remota & $\begin{array}{l}\text { Si riconosce il pensionamento come parte della propria vita lavorativa, ma tale evento è rimosso in un } \\
\text { lontano futuro }\end{array}$ \\
\hline Prossima & $\begin{array}{l}\text { Si avvertono le possibili difficoltà nell'adattamento al ritiro dall'attività lavorativa, nella consapevolezza } \\
\text { di dover affrontare una nuova realtà nel breve termine }\end{array}$ \\
\hline Della luna di miele & $\begin{array}{l}\text { Prevale l'euforia di potersi dedicare finalmente a sé stessi, ai propri hobby, ai viaggi e a tutte le cose } \\
\text { che si volevano fare in precedenza }\end{array}$ \\
\hline Del disincantamento & $\begin{array}{l}\text { Si prende coscienza dei limiti del nuovo status di pensionato, quali la perdita degli amici, colleghi di } \\
\text { lavoro, le possibili difficoltà economiche, lo stato di salute mediocre, eccetera }\end{array}$ \\
\hline Del ri-orientamento (della stabilità) & Si accetta il pensionamento, anche con i limiti da esso imposti, in una vita stabilizzata e riorganizzata \\
\hline Finale & Comprende il periodo della vera e propria senescenza, con le implicazioni ad essa correlate
\end{tabular}

Impatto negativo

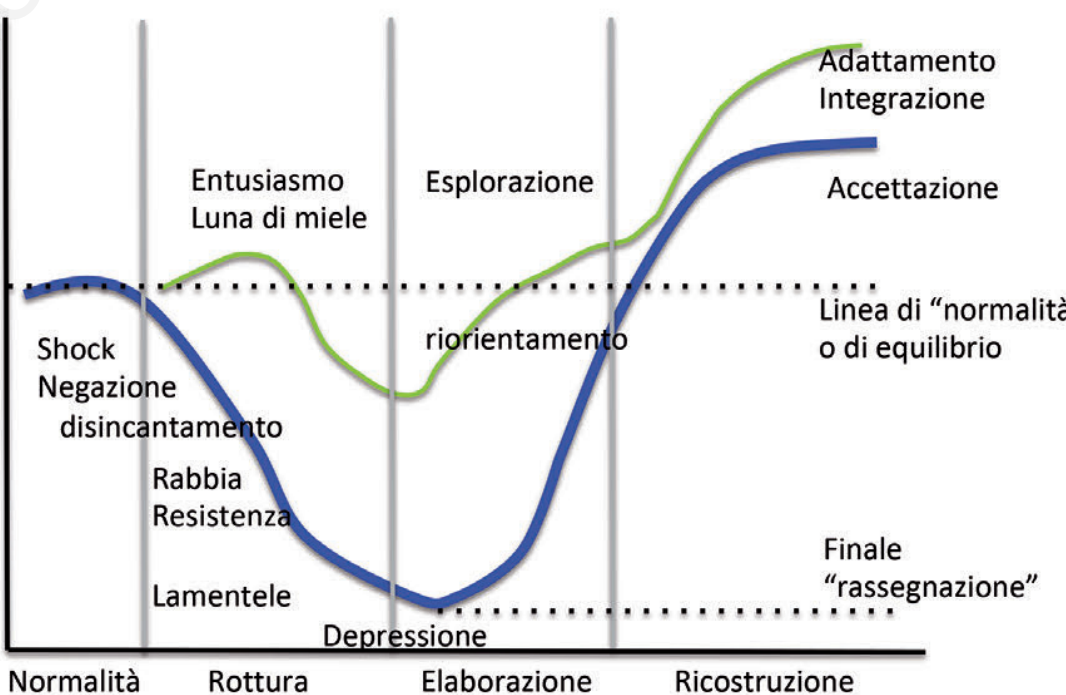

Figura 1. Le fasi di adattamento al pensionamento. (Modificata da Santucci ${ }^{12}$ ). 
è soggetto ed oggetto di produzione e consumo. La vita stessa di tutti noi si è orientata in tal senso, con una prima fase di apprendimento-formazione, un periodo successivo di attività lavorativa produttiva ed una fase di riposo, corrispondente al pensionamento. L'identificazione della propria esistenza soltanto sul lavoro può comportare, al momento della cessazione dell'attività lavorativa e la conseguente perdita del proprio ruolo, un'occasione di crisi, aggravata dal contesto sociale, che tende a privilegiare le persone produttive rispetto a quelle improduttive. ${ }^{6,13}$ In tal modo il pensionato rischia di diventare socialmente un vecchio, anche se biologicamente non lo è ancora. ${ }^{14} \mathrm{La}$ crisi del ruolo o dell' $i$ dentità, con la perdita del prestigio lavorativo e professionale può indurre un abbassamento della stima di sé, con riduzione della partecipazione sociale dell'individuo e possibile deterioramento cognitivo conseguente. Al contrario, nella continuità dell'identità personale, dando più spazio alle sfere personale e sociale spesso trascurate negli anni dell'attività lavorativa, si può realizzare un adattamento graduale al pensionamento, con poche conseguenze sulla qualità della vita, le relazioni famigliari, amicali ed interpersonali.

\section{Pensionamento volontario e pensionamento obbligato}

Del mio lavoro non mi mancano il caos, la burocrazia, le inequità e la pianificazione non condivisa, spesso effettuata da persone dominanti incompetenti, con un clima insopportabile.

Esiste differenza fra pensionamento volontario e pensionamento obbligato, inteso come dipendente da impellenti motivi di salute, problemi economico-finanziari del datore di lavoro, cambiamenti dei contesti lavorativi o da obblighi normativi e di legge. Tali differenze sono state ben descritte nel sesso maschile, ${ }^{15}$ ma possono essere ugualmente riferite anche a quello femminile (Tabella 2).

Analisi longitudinali suggeriscono che lo stato di salute migliora dopo il pensionamento, sia obbligato che volontario, anche se il miglioramento sembra at- tenuarsi nel tempo. Al contrario, l'associazione tra pensione determinata da malattie preesistenti e precaria salute sembra riflettere una selezione di casi piuttosto che un diretto rapporto causa-effetto tra cessazione dell'attività lavorativa e e prognosi. ${ }^{16} \mathrm{Si}$ pone, peraltro, un legittimo quesito: il pensionamento obbligatorio ope legis è oggi una norma socialmente equa, non scevra da privilegi e costituzionalmente accettabile? Si richiama, a tal proposito, l'esempio dei Professori universitari che, a differenza dei Primari ospedalieri, godono nel SSN di un diritto di pensionamento dilazionato fino a 70 anni. Su questo tema esistono evidenze contradditorie. La prevalenza di sindrome ansioso depressiva nei pensionati obbligati sembra essere inferiore rispetto ai pensionati di libera scelta. ${ }^{17}$ Nei pensionati ex dipendenti pubblici esiste una quota rilevante di pazienti depressi. ${ }^{6}$ Anche se il pensionamento non sembra in grado di aumentare il rischio di gravi malattie croniche, il ritiro dal lavoro è stato associato in ex dipendenti delle aziende francesi del gas ed elettriche - pensionati non per ragioni di salute - ad una sostanziale riduzione dell'affaticamento mentale e fisico e dei sintomi depressivi, particolarmente in coloro che avevano una malattia cronica. ${ }^{18}$ Secondo altri dati il pensionamento aumenta significativamente il rischio di una diagnosi successiva di malattia cronica, prevalentemente di tipo cardiovascolare o neoplastica. ${ }^{19}$ I pensionati volontari mostrano atteggiamenti positivi rispetto a quelli non volontari: i fattori predittivi di una miglior soddisfazione dopo il pensionamento sono il precedente stato di salute e le considerazioni soggettive pre-pensionistiche sul ritiro dal lavoro. ${ }^{20}$ Secondo le ventisettesima Retirement Confidence Survey risulta che i fattori più utili per aumentare la produttività sul lavoro sarebbero la pianificazione della pensione (53\%), la pianificazione finanziaria (49\%) o i programmi di assistenza sanitaria (47\%). Dopo un pensionamento obbligato, vivono meglio la loro condizione coloro che: i) impiegano l'aumentato tempo libero per ottimizzare la propria salute; ii) sanno mantenere buoni contatti sociali; iii) continuano a svolgere un'attività fisica regolare; iv) godono di una buona assistenza e/o di assicurazione sanitaria; v) vivono in un contesto di un buon welfare state. ${ }^{21}$

Tabella 2. Differenze fra pensionamento volontario ed obbligato.

\begin{tabular}{ll}
\hline Pensionamento volontario & Pensionamento obbligato \\
\hline Migliore appagamento emozionale & Brusco cambiamento nello stile di vita \\
\hline $\begin{array}{l}\text { Più frequente sensazione di serenità } \\
\begin{array}{l}\text { Migliore pianificazione (attività occupazionali, tempo libero, } \\
\text { interessi economici, eccetera) }\end{array}\end{array}$ & Penso di inutilità, ridotta fiducia ed immagine negativa di sé \\
\hline $\begin{array}{l}\text { Minore prevalenza di stati depressivi, di problemi affettivi e } \\
\text { di conflittualità }\end{array}$ & $\begin{array}{l}\text { Sentimenti prevalenti di debolezza, ostilità, distacco ed isolamento } \\
\text { sociale }\end{array}$
\end{tabular}




\section{È auspicabile la prosecuzione dell'attività lavorativa in età più avanzata?}

\author{
Il lavoro salva l'uomo da tre grandi mali: \\ noia, vizio e necessità ${ }^{2}$
}

L'aumentata speranza di vita può indurre le persone a cercare di prolungare l'attività lavorativa anche dopo il pensionamento. Tale scelta si basa su diverse motivazioni, a favore e contro, oltre a vari ostacoli oggettivi (Tabella 3) ${ }^{23}$ Posticipare la rendita di vecchiaia può essere un'opportunità da non sottovalutare, tenendo conto anche del fatto che chi gode di buona salute può ancora garantire determinati lavori, rendendosi sempre più spesso disponibile a continuare la sua attività anche dopo l'età del pensionamento.

Il ritiro dal lavoro corrisponde ad una fase beata della vita o no? Cosa pensare degli effetti del pensionamento sulla salute? È meglio andare in pensione o semplicemente continuare a lavorare? Qual è la cosa più salutare da fare?. ${ }^{24,25}$ Fino a pochi anni fa nella maggior parte dei paesi solo una minoranza di lavoratori continuava a lavorare dopo i 55-60 anni e questi erano in media più sani di quelli che andavano in pensione (healthy worker effect). Ancora oggi le persone che lavorano, pur percependo la pensione, sono poche ( $21 \%$ del totale dei percettori di pensioni di vecchiaia con meno di 75 anni) e coloro che lavorano oltre l'età di pensionamento sono solamente circa il $6 \%$ del totale delle persone fra i 66 e i 75 anni. ${ }^{26}$ La capacità lavorativa degli anziani, pur se oggetto di pochi studi, sembra modificarsi in maniera differente, a seconda che vengano considerate le capacità fisiche rispetto $\mathrm{a}$ quelle mentali, pur con alcune differenze nelle diverse casistiche. In generale, il declino delle capacità mentali e sociali è più lento e più tardivo di quello delle capacità fisiche, anche se con l'età aumenta la prevalenza di disturbi comuni, soprattutto ansia e depressione. ${ }^{27}$ In uno studio effettuato su più di 16.000 uomini e donne è stato documentato - pur con qualche limite metodologico - un aumento del 51\% nella mortalità per tutte le cause, principalmente da malattie cardiovascolari dopo il ritiro dall'attività lavorativa, mentre una dilazione di 5 anni del pensionamento si associava ad una diminuzione del $10 \%$ della mortalità. Secondo questa ricerca, in sostanza, risulterebbe che

Tabella 3. Elementi a favore e contro alla prosecuzione dell'attività lavorativa dopo il pensionamento.

\begin{tabular}{|c|c|c|}
\hline Pro & Contro & Ostacoli \\
\hline $\begin{array}{l}\text { La produttività dei lavoratori anziani } \\
\text { è sovrapponibile a quella dei giovani }\end{array}$ & $\begin{array}{l}\text { Età cronologica (non biologica) come } \\
\text { limite discriminante rispetto al } \\
\text { mantenimento dell'attività lavorativa }\end{array}$ & Pregiudizi e stereotipi sulla vecchiaia \\
\hline $\begin{array}{l}\text { La maggioranza dei lavoratori anziani } \\
\text { non presenta disabilità fisiche tali da } \\
\text { impedire una qualche attività lavorativa }\end{array}$ & $\begin{array}{l}\text { Politica aziendale finalizzata a mantenere } \\
\text { un'immagine giovanilistica dell'industria }\end{array}$ & $\begin{array}{l}\text { Conflitti generazionali e discriminazioni legate all'età } \\
\text { anagrafica }\end{array}$ \\
\hline $\begin{array}{l}\text { L'assenteismo tra i lavoratori anziani } \\
\text { non è superiore a quello delle altre fasce } \\
\text { di età }\end{array}$ & $\begin{array}{l}\text { Supervalutazione delle capacità lavorative } \\
\text { dei giovani rispetto a quelle degli anziani }\end{array}$ & $\begin{array}{l}\text { Mancanza di protezione sociale ed inadeguata } \\
\text { sicurezza lavorativa }\end{array}$ \\
\hline $\begin{array}{l}\text { La maggior esperienza dei lavoratori } \\
\text { anziani costituisce un valore da tutelare }\end{array}$ & $\begin{array}{l}\text { Ridotto interesse e demotivazione in età } \\
\text { più avanzata }\end{array}$ & $\begin{array}{l}\text { Limiti determinati dall'invecchiamento e dalle } \\
\text { malattie presenti }\end{array}$ \\
\hline $\begin{array}{l}\text { La maggior esperienza dei lavoratori } \\
\text { anziani li rende meno esposti agli } \\
\text { infortuni }\end{array}$ & $\begin{array}{l}\text { Ridotta attitudine agli sforzi fisici e } \\
\text { modificazione dei tempi di lavoro } \\
\text { in relazione alle modificazioni correlate } \\
\text { all'invecchiamento }\end{array}$ & Ridotta capacità di mobilità \\
\hline $\begin{array}{l}\text { I lavoratori anziani garantiscono spesso } \\
\text { maggiore assiduità, diligenza e precisione } \\
\text { nel lavoro }\end{array}$ & $\begin{array}{l}\text { Scarsa propensione ai cambiamenti, } \\
\text { mentalità conservatrice, resistenza e/o } \\
\text { rifiuto all'innovazione, scarsa tendenza } \\
\text { alla mobilità }\end{array}$ & $\begin{array}{l}\text { Limitata libertà di scelta qualora sia necessario un } \\
\text { cambiamento della mansione }\end{array}$ \\
\hline $\begin{array}{l}\text { Gli anziani, con adeguato training, sono } \\
\text { comunque in grado di acquisire nuove } \\
\text { qualificazioni ed implementare nuove } \\
\text { procedure }\end{array}$ & $\begin{array}{l}\text { Ridotta propensione ai cambiamenti, } \\
\text { mancanza d'iniziativa, diminuita } \\
\text { capacità di apprendimento su nuove } \\
\text { tecnologie }\end{array}$ & $\begin{array}{l}\text { Offerta di mansioni di livello minore, meno } \\
\text { remunerative e di scarsa soddisfazione }\end{array}$ \\
\hline $\begin{array}{l}\text { L'esperienza pratica è spesso più utile } \\
\text { delle conoscenze teoriche }\end{array}$ & $\begin{array}{l}\text { Conflitti generazionali, disoccupazione } \\
\text { giovanile, contingenze economiche }\end{array}$ & $\begin{array}{l}\text { Legislazione pensionistica rigida, non flessibile, } \\
\text { ma soggetta a decisioni politiche sempre mutevoli }\end{array}$ \\
\hline $\begin{array}{l}\text { Maggiori conoscenze, competenze, } \\
\text { abilità specifiche, orientamento al } \\
\text { problem solving }\end{array}$ & $\begin{array}{l}\text { Limitazione nelle attività fisiche e mentali, } \\
\text { maggiore vulnerabilità a stress correlato } \\
\text { al lavoro, frequenti insonnia e depressione }\end{array}$ & \\
\hline
\end{tabular}


il pensionamento può essere un fattore di rischio per la mortalità per tutte le cause e per quella cardiovascolare in particolare.$^{28} \mathrm{Da}$ alcune indagini sperimentali si evince che il ritiro da una vita attiva può avere conseguenze negative sulle funzioni cognitive, ${ }^{29}$ con compromissione accelerata della memoria verbale. ${ }^{30}$ Si evidenzia una maggior suscettibilità dei soggetti di oltre 45-50 anni ai rischi correlati ad un elevato impegno ergonomico, con aumentata incidenza di disturbi muscolo-scheletrici. ${ }^{31-33} \mathrm{Il}$ declino funzionale si accentua più rapidamente durante il pensionamento rispetto all'attività lavorativa a tempo pieno in dipendenti di età pari o superiore a 65 anni e la differenza non è spiegata dall'assenza di malattie croniche e di rischi legati allo stile di vita. ${ }^{34}$ Lavorare oltre l'età pensionabile può essere utile per la salute mentale, grazie al mantenimento di un ruolo sociale produttivo e ai vantaggi economici, ma è improbabile che i benefici dell'occupazione post-pensionamento siano universali, proprio in ragione delle differenti caratteristiche e propensioni soggettive, nonché delle diverse condizioni di lavoro individuali. ${ }^{35}$ I potenziali benefici dell'estensione dell'attività lavorativa oltre i limiti imposti dalle norme devono essere considerati alla luce del contesto economico-sociale e delle motivazioni individuali. Da uno studio dell'Università Ca' Foscari di Venezia risulta che, particolarmente in condizioni di crisi economica, il pensionamento può essere vissuto come un agognato sollievo da condizioni di precarietà e incertezza. Ciò sembra riguardare particolarmente gli operai di sesso maschile, negli ultimi anni di vita lavorativa, con peggioramento dello stato affettivo ed aumentato rischio di depressione, in relazione al crescente timore di perdere il lavoro, senza poter trovare una nuova posizione alternativa. ${ }^{36}$ Nella tendenziale propensione a lavorare più a lungo e a dilazionare l'età obbligatoria del pensionamento è importante che gli anziani mantengano il controllo soggettivo - ovvero non etero-imposto - sulla propria decisione di lavorare in età più avanzata. ${ }^{37}$ Secondo altri dati, gli anziani occupati mostrano uno stato di salute migliore rispetto ai disoccupati, particolarmente quando impegnati in attività richiedenti un maggior impegno fisico: gli operai mostrano un minor rischio di rischio di multi-morbilità e limitazione funzionale. ${ }^{38}$ Un recente studio giapponese, effettuato su oltre 15.000 persone, ha dimostrato che strategie finalizzate a lavorare più a lungo possono consentire di vivere meglio, contribuendo ad un'aumentata speranza di vita in miglior salute. ${ }^{39}$ In sostanza, le riforme che inducono le persone a rinviare il pensionamento non sono importanti solamente per una migliore solvibilità dei sistemi pensionistici, ma, col tempo, potrebbero anche rendere un dividendo per la salute degli anziani, migliorando la qualità di vita delle persone e riducendo i costi dell'assistenza sanitaria pubblica. ${ }^{40} \mathrm{La}$ possibilità di proseguire il rapporto lavorativo oltre l'età pensionabile di vecchiaia era, del resto, una delle prerogative della Riforma Fornero che all'articolo 24, comma 4 del Dl 201/2011 aveva incentivato i lavoratori a rimanere sul posto di lavoro oltre il compimento dell'età pensionabile, sino ai 70 anni. ${ }^{41}$ Con la sentenza n. 20089 del 30.07.2018, la Cassazione ha affermato che l'art. 24, comma 4, del D.Lgs. 201/2011 non riconosce al lavoratore alcun diritto soggettivo a rimanere in servizio fino al compimento del settantesimo anno d'età, prevedendo soltanto tale circostanza come mera possibilità da concordare con l'azienda datrice. Stanti i problemi di incertezza che determina l'attuale quadro normativo sarebbe preferibile ricercare accordi di risoluzione consensuale con i singoli lavoratori che, via via, si vengono a trovare in possesso dei requisiti di pensionabilità richiesti per legge.

\section{Pensionarsi prima può far spazio al lavoro dei giovani?}

Il lavoro si basa su forze multi-generazionali, caratterizzate da lavoratori afferenti sostanzialmente a quattro tipologie: i) quelli più anziani (i nati tra il 1928-1945); ii) i boomers (nati dal 1946 al 1964); iii) la generazione X (nati tra il 1965 e il 1980); iv) generazione $Y$ (millennials), nati dopo il 1980, questi ultimi gravati dal dover affrontare la recessione economica dopo il 2007. Mandare in pensione sempre più tardi i lavoratori anziani ancora occupati evoca il legittimo dubbio che tale scelta sia a detrimento dei giovani alla ricerca di un'occupazione stabile. In contrasto con l'ipotesi dell'utilità di una solidarietà espansiva determinata dalla staffetta generazionale vengono addotte almeno tre controdeduzioni: i) nei Paesi in cui si va in pensione più tardi il tasso di occupazione dei giovani non è inferiore rispetto agli altri (anzi risulta mediamente più alto); ii) ritardare l'innalzamento dell'età pensionabile comporterebbe un innalzamento dei contributi sociali, misura assai gravosa per gli stessi giovani (sia sotto forma di minore occupazione che di buste paga più leggere); iii) i posti di lavoro lasciati liberi dagli anziani potrebbero semplicemente sparire, per decisioni politiche e/o aziendali di ridotto turn over, ovvero non essere ricopribili da parte di lavoratori giovani, cui spesso mancano le competenze e l'esperienza dei lavoratori più anziani. ${ }^{42}$ Gli economisti del lavoro hanno infatti dimostrato non solo che è infondata la convinzione che le opportunità occupazionali per i giovani possano aumentare se si prepensionano gli anziani. In presenza di difficoltà economiche può prevalere la regola del last in first out: se la domanda diminuisce, le imprese tendono a favorire la componente della forza lavoro in grado di offrire maggiore qualità e competenza, rispetto all'obbligo di formazione e di tutoraggio dei più giovani. ${ }^{43}$ 
Togliere il lavoro agli anziani per darlo ai giovani, in sostanza, non servirebbe, dato che le aziende non risparmiano un centesimo e non migliorano le loro capacità concorrenziali e produttive con la sostituzione di personale specializzato con giovani inesperti. Va ricordato infine che l'anticipazione della data di pensionamento comporta costi rilevanti: è stato calcolato che in un sistema finanziato a ripartizione (vedi paragrafo successivo) per coprire il costo di un anno di pensione di anzianità goduta in anticipo (mediamente corrispondente a circa 29 mila euro lordi annui), occorrono i contributi sociali versati da ben 5 lavoratori dipendenti giovani: una retribuzione media mensile di 1100 euro nette dà luogo a circa 5600 euro annui di contributi previdenziali a carico del lavoratore e dell'impresa. ${ }^{44}$

I giovani di oggi andranno in pensione a più di 70 anni. Tale dato, non solamente italiano, si evince dal report dell'Organizzazione per la Cooperazione e lo Sviluppo Economico. Secondo le proiezioni dell'OECD, in Italia i giovani che nel 2016 avevano 20 anni andranno in pensione a 71 anni. Secondo questo report, i giovani di danimarca, Italia e Olanda sono quelli che andranno in pensione più tardi, mentre $\mathrm{i}$ giovani di Turchia, Slovenia e Lussemburgo sono quelli che andranno in pensione prima. ${ }^{45}$ Secondo le valutazioni dell'INPS, a causa di un vuoto contributivo e della disoccupazione, l'età pensionabile di chi è nato dopo il 1980 può slittare anche fino a 75 anni. ${ }^{46}$ Lo spostamento in avanti dell'età pensionabile fa emergere un quesito: quando mai i giovani potranno usufruire di una pensione e cosa devono fare per raggiungere almeno 20 anni di contributi versati, che almeno garantiscono un diritto minimo quando si raggiungerà l'età? Oltre ai progetti individuali di capitalizzazione, il riscatto della laurea può essere utile per accelerare l'accesso alla pensione, anche se relativamente oneroso, pur se attivato precocemente. Il riscatto della laurea è uno strumento riconosciuto dall'INPS, rateizzabile e deducibile per gli oneri fiscali, con cui valorizzare ai fini pensionistici il periodo del proprio percorso formativo, ma solamente a seguito di un documentato conseguimento del titolo di studio (https://www.money.it/riscatto-laurea-comefunziona-quanto-costa-conviene-calcolo).

\section{I sistemi pensionistici a ripartizione e a capitalizzazione}

Pagare i contributi per i pensionati di oggi (la ripartizione): Nel sistema a ripartizione i lavoratori attuali pagano i contributi per le pensioni di coloro che hanno smesso di lavorare. In sostanza, la pensione di tutti noi sarà pagata dai lavoratori di domani: l'equilibrio di questo sistema solidale si basa su un giusto rapporto tra il numero dei contribuenti e il numero di pensionati. La maggioranza dei Paesi europei, tra cui l'Italia, si basa su un sistema di questo tipo.
Risparmiare oggi per la propria pensione di domani (la capitalizzazione): La capitalizzazione presuppone che ciascun lavoratore versi denaro per la propria futura pensione. La somma raccolta e il tasso d'interesse determineranno l'importo finale. La capitalizzazione può essere fatta individualmente (i fondi vengono investiti in un conto bancario o in immobili) $\mathrm{o}$ affidata ad un gestore privato (fondi di investimento o fondi pensione).

\section{Chi vorrebbe continuare a lavorare anche dopo l'età della pensione ha solamente motivazioni economiche?}

Uno studio del CENSIS aveva mostrato che la maggioranza degli ultra-sessantenni desidera continuare a lavorare, sia per il reddito aggiuntivo che per non invecchiare: il lavoro diventa così uno strumento per l'autotutela e la realizzazione personale dell'anziano ${ }^{47}$ Entrano in gioco vari fattori, quali il grado d'istruzione più elevato, le migliorate condizioni di salute e la riduzione delle invalidità sino ad età più avanzate, l'interesse per il lavoro e il desiderio di mantenere una qualche responsabilità, il timore dell'inattività, dell'ozio e del senso di inutilità, la voglia di mantenere potere e prestigio, se non la necessità, di conservare relazioni sociali e professionali. Le motivazioni economiche riguardano prevalentemente individui di basso reddito, spesso inferiore al minimo vitale, che continuano a lavorare anche in condizioni fisiche non ottimali. In altri casi si tratta di persone che amano il proprio lavoro e che vogliono ancora sentirsi utili. Per questi ultimi l'attività svolta in condizioni di libera scelta rappresenta un fattore benefico, con vantaggi che si esprimono anche sulla sfera biologica. È evidente che tali opzioni dipendono da diverse variabili (stato di salute, anni complessivi di attività lavorativa svolta, carico lavorativo, contesto e bisogni della famiglia, clima organizzativo e qualità nei rapporti con i colleghi, capacità di adattamento dei lavoratori più anziani all'informatizzazione e alla gestione digitale delle attività, eccetera) e, soprattutto, dal tipo di mansione esercitata. È legittimo sostenere che i lavori gravosi o usuranti (di cui, in Italia, all'articolo 2 del decreto Ministero del Lavoro del 19 Maggio 1999-c.d. Decreto Salvi e D.lgs 67/2011) sono quelli che vengono abbandonati più volentieri, mentre, al contrario, si tende a mantenere attività dirigenziali, professionali ed intellettuali, ben più gratificanti, non solo sul piano economico. La maggior parte delle ricerche sul rapporto tra salute e pensionamento ha prodotto risultati ambigui. È possibile che la salute inizialmente migliori quando si va in pensione, poi, dopo un po', inizia a deteriorarsi a causa della ridotta attività fisica e delle diminuite interazioni sociali. Il pensionamento, oltre ad una rilevante prevalenza di depressione, ${ }^{6}$ può 
comportare anche una percezione soggettiva di ridotta salute fisica e mentale, con maggior ricorso ai farmaci, tanto da far auspicare un'elevazione dell'età obbligatoria di pensionamento, anche alla luce dell'aumentata aspettativa di vita. ${ }^{48}$

\section{Pensionamento flessibile e pensionamento progressivo}

Il timing e le modalità di pensionamento sono elementi importanti per l'adattamento delle persone al ritiro dall'attività lavorativa. ${ }^{49}$ Secondo un'indagine Eurobarometer un terzo degli europei vorrebbe continuare a lavorare dopo l'età della pensione, ritenendo due terzi degli europei - che un lavoro a tempo parziale combinato con una pensione parziale è più attraente di un pieno pensionamento senza attività. Circa il $27 \%$ degli europei di età pari o superiore a 55 anni svolge una qualche forma di attività, comprese quelle di volontariato in varie organizzazioni, dedicandovi in media 14 ore al mese..$^{50}$ Il tema della adattabilità, spesso associato al termine modernizzazione, si ritrova spesso nei rapporti e nei documenti della Commissione Europea, in base alla convinzione che un sistema pensionistico dove i singoli individui possono avere un certo margine di scelta sull'età di ritiro sia più adeguato per rispondere alle rapide trasformazioni di una moderna società. ${ }^{51} \mathrm{Il}$ concetto di flessibilità in tema di pensionamento dovrebbe presupporre il superamento della mera età cronologica come età fissata ope legis, consentendo al singolo individuo una maggior libera scelta su quando ritirarsi dal lavoro, in funzione della vera età bio-fisiologica e dell'idoneità psico-fisica, dei desideri personali, nonché nell'ambiente di lavoro, dell'assenza di controindicazioni ambientali e soggettive. Nel pensionamento progressivo si propone, anche alla luce dell'aumentata speranza di vita, un'alternativa all'interruzione brusca del lavoro, con lo shock da pensionamento che ne può conseguire. Viene data la possibilità di ampliare il periodo di adeguamento alla nuova condizione con periodi di interruzione, alternando attività ed inattività secondo periodi programmati, riducendo le ore lavorative settimanali (il cosiddetto semi-retire, lavorando, ad esempio quattro giorni alla settimana), oppure prolungando i periodi di ferie, recuperando quelle non godute. ${ }^{52}$ Alcuni lavoratori ottengono di poter modificare il proprio orario di lavoro, onde disimpegnarsi gradualmente dall'attività professionale avvicinandosi l'epoca del pensionamento, ma non esistono, in generale, regole formali di questo tipo, anche se sembra crescere l'interesse da parte delle aziende per una qualche forma di pensionamento progressivo. ${ }^{53}$ Proprio perché una popolazione anziana infelice pesa e peserà sempre di più - anche in termini economici - sulla società, è necessario preoccuparsi d'introdurre nuovi metodi di prevenzione per evitare la marginalizzazione delle persone di età avanzata. Tutto ciò pone evidenti problemi di sostenibilità, per cui sono necessarie politiche condivise tra decisori istituzionali, parti sociali, enti locali ed intermedi a favore di un invecchiamento di successo. ${ }^{54} \mathrm{Ai}$ fini della sostenibilità, i sistemi pensionistici in Europa dovrebbero incoraggiare una più lunga permanenza nel mondo del lavoro, facilitando metodi per l'estensione della vita lavorativa (obiettivo 5) attraverso l'aumento dell'occupazione nella fascia tra 55 e i 64 anni ed una diversa concezione del pensionamento anticipato, che non dovrebbe più essere utilizzato come un mezzo per creare occupazione a beneficio dei più giovani o per ridurre la disoccupazione. Negli ultimi tempi, sempre più economisti ritengono che la crisi della zona-euro obbligherà ad un innalzamento dell'età di pensionamento fino a circa 80 anni. ${ }^{55} \mathrm{Negli}$ Stati Uniti non c'è un'età legale in cui un americano può o dovrebbe andare in pensione: si può scegliere di andare in pensione quando si vuole, generalmente tra $\mathrm{i}$ 62 e i 70 anni, ${ }^{56}$ ma dobbiamo ricordare che negli USA tutti sono pressoché obbligati a ricorrere a pensioni integrative individuali private. Negli USA (e non solo) si lavorerà fino a 80 anni, ma è stato evidenziato che il prolungamento della carriera finanche a tale età, se non associato a versamenti del proprio stipendio durante la vita lavorativa, non è in grado di garantire un tenore di vita soddisfacente e che questo viene fortemente condizionato dal precedente reddito annuo. Coloro che hanno percepito uno stipendio medio nel corso della carriera sotto gli 11.700 dollari all'anno dovrebbero rimandare il pensionamento a quando compiono 84 anni prima che il $90 \%$ di loro possa raggiungere almeno il $50 \%$ della possibilità di permettersi di non lavorare più. La fascia tra gli 11.700 e i 31.200 dollari, per avere il $50 \%$ di chance di coprirsi le spese di base per una vecchiaia dignitosa, dovrebbe continuare ad andare in fabbrica o in ufficio fino ai 76 anni. La soglia scende a 72 anni per chi ha una media di introiti tra $i$ 32.200 e i 72.500 , e si riduce ai 65 anni per coloro che superano i 72.500 di media annua. ${ }^{57}$ In Europa la modernizzazione dei sistemi pensionistici dovrebbe tener conto dei cambiamenti del mercato del lavoro (obiettivo 9), adattandosi a modelli di occupazione e di carriera flessibili, con opportuni strumenti incentivanti e/o non premianti, incoraggiando carriere lavorative più lunghe e disincentivando uscite precoci dal mercato lavoro. ${ }^{58}$ Diverse modalità possono essere implementate per facilitare il pensionamento progressivo: ${ }^{59}$ i) un'organizzazione del lavoro basta sulle classi di etàda una certa età in poi distribuire il lavoro, massimizzando l'apporto di esperienza e riducendo il carico psico-fisico dei lavoratori più anziani; ii) un percorso di flessibilità in uscita verso la pensione che si può realizzare con differenti interventi, purché universali, standardizzati, ben calibrati e non discrezionali. È importante garantire un pensionamento flessibile senza disuguaglianze sanitarie 
e sociali tra gli anziani e tra anziani e giovani. La promozione della formazione e degli interscambi fra generazioni di lavoratori, fino a far sfumare i confini tra le classi d'età nei luoghi di lavoro, è uno degli strumenti auspicati per perseguire un rinnovato equilibrio intergenerazionale. ${ }^{60,61}$ Un periodo intermedio di sovrapposizione anziani/giovani, nell'ambito di un pensionamento progressivo e di apprendistato, di studio e di osmotico, rispettivamente per gli uni e gli altri, potrebbe facilitare un percorso di ricambio generazionale, utile per le aziende e i soggetti sociali. Un modo tradizionale di trasmettere l'esperienza per imparare insieme il mestiere, che, forse, nel tempo, potrebbe disperdersi. In sostanza con l'impegno reciproco, lo studio e l'interazione si avvantaggiano tutti: gli apprendisti giovani per imparare il lavoro dall'esperienza degli anziani, gli anziani per trarre dai giovani l'energia vitale ed un rinnovato entusiasmo per adeguarsi ad una nuova esistenza, con la soddisfazione di aver lasciato l'eredità di una competenza specifica, utile non solo per l'Azienda, ma anche per il nostro Paese.

\section{Fattori di rischio di un pensionamento infelice}

Ci ritiriamo quando non ce la facciamo più? Quando andiamo in pensione, l'unica energia che ci rimane è quella di camminare verso la fine?

Prendere, usare, gettare. Nella società consumistica con l'uomo si fa altrettanto: lo si prende per lavorare, lo si utilizza ed alla fine lo si getta ed emargina, ${ }^{62}$ con una dimensione umana che è andata perduta. La persona che va incontro alla senescenza dovrebbe, invece, guardare sempre avanti alla ricerca di un invecchiamento il meno infelice possibile. Secondo Bridget Melton, della Georgia Southern University i capisaldi di un invecchiamento di successo, per traslato di un pensionamento felice, sono: i) evitare malattie gravi e debilitanti e disabilità; ii) impegnarsi nella maggior parte delle normali attività della vita quotidiana; iii) mantenere la funzione cognitiva; iv) essere in grado di affrontare cambiamenti fisici, sociali ed emotivi; v) possedere un senso di controllo della propria vita ${ }^{63}$ Diversi fattori sono correlati ad un pensionamento infelice, categorizzabili in 4 dimensioni: mediche, psico-dinamiche, sociali ed economiche, sintetizzate nella Tabella 4.

Sulla base dei diversi fattori riportati in tabella, consideriamo alcuni elementi salienti.

\section{Fattori medici}

\section{Depressione e pensionamento}

I sintomi della depressione sono comuni nella vecchiaia: $1{ }^{\prime} 8 \%-20 \%$ delle persone anziane ha riferito di stati depressivi o sintomi depressivi negli studi epidemiologici. La depressione, a sua volta, altera la qualità della vita, aumenta i costi dell'assistenza sanitaria ed è associata a mortalità prematura. Le avversità in età infantile, gli eventi negativi nella mezza età, lo stress coniugale, ma anche le difficoltà socio-economiche e psicosociali (come lo stress da lavoro), valutati in età avanzata hanno dimostrato di avere effetti a lungo termine sulla depressione in età avanzata. Tuttavia, la misura in cui le esposizioni durante l'impiego hanno un valore predittivo dopo la pensione non è stata stabilita. Gli studi sui sintomi depressivi come risultato post-pensionamento dovrebbero tener conto, comunque, del contributo di una cattiva salute fisica e dei comportamenti a rischio per la salute in età adulta e avanzata, poiché sono fonti plausibili di bias o causalità inversa, dato che depressione, malattie fisiche e comportamenti a rischio sanitario, tendono a raggrupparsi nel stessi individui. L'assenza di malattie precedenti o la presenza di una sola malattia sembra associarsi significativamente $(\mathrm{P}=0,02)$ ad assenza di depressione nel post pensionamento, mentre almeno 2 malattie si osservano più spesso nei depressi dopo il pensionamento. I depressi presentano più frequentemente una storia di cardiopatia $(\mathrm{P}<0,005) .{ }^{6}$ Sono associati a un elevato rischio di depressione, oltre ai fattori genetici, episodi depressivi ricorrenti, co-morbilità fisica e psichiatrica. ${ }^{64}$

\section{Il rifugio in sostanze voluttuarie}

Un aumento del consumo di alcol a seguito del pensionamento può verificarsi a seguito della perdita dello status sociale e d'identità, ovvero ad una perdita di scopo. Gli adulti di età superiore ai 55-60 anni che consumano $5 \mathrm{o}$ più bevande in un giorno o più di 7 bevande a settimana hanno più probabilità di manifestare sintomi correlati all'alcol rispetto agli adulti più anziani che consumano meno alcolici ${ }^{65}$ (l'American Geriatric Society raccomanda non più di 7 drink a settimana e/o 2 bevande per ogni occasione per gli adulti oltre i 65 anni) ${ }^{66}$ Anche piccole quantità di alcol possono avere un impatto significativo sulla salute. L'abuso etilico può esprimersi in diverse forme: i) $\mathrm{mi}$ suse (bere fino al punto di ubriacarsi); ii) binge drinking (bere periodicamente $>4$ drink per gli uomini e $>3$ per le donne); iii) alcol abuse (bere nonostante gli effetti avversi sulla salute, sull'interazione sociale, sulla performance quotidiana; iv) alcool dependence (brama di alcool, controllo alterato, o sintomi di astinenza). L'alcol è la sostanza voluttuaria più comune utilizzata dagli anziani: il 17\% circa di bevitori ha problemi correlati all'alcool, con il 22\% degli accessi ospedalieri o di pronto soccorso. ${ }^{67,68}$ Uno studio del 2014 sul tema Alcoholism Clinical and Experimental Research, basato sui sondaggi della National Health Interview Survey su 65.303 persone, ha evidenziato che il consumo di alcol è in aumento tra gli anziani in toto, in particolare nel sesso femminile (tra il $1997 \mathrm{e}$ il 2014 dello 0,7 percento all'anno tra i maschi, 1,6 
percento tra le donne). A parità di volume medio nel tempo, il binge drinking, stabile per gli uomini, è aumentato per le donne del 3,7 percento all' anno). ${ }^{69}$ Uno studio del 2009 sull'American Journal of Psychiatry ha rilevato, inoltre, che tra gli adulti tra i 75 e gli 85 anni, circa il $27 \%$ delle donne e uno sconcertante $49 \%$ degli uomini beve oltre le raccomandazioni per la loro età fornite dalle linee guida. ${ }^{70}$ Altri studi, al contrario, non hanno rilevato alcun cambiamento o addirittura una riduzione del consumo di alcolici post pensionamento, in quanto le persone sperimenterebbero meno stress (correlato prima al lavoro) e ridurrebbero i le- gami sociali con i colleghi che incoraggiavano a bere..$^{71-73}$ In sostanza, il ritiro dall'attività lavorativa non sembra, di per sé, correlarsi direttamente all'abuso di alcol: sono piuttosto le condizioni di contesto e le esperienze prima e dopo il pensionamento a condizionare possibili comportamenti voluttuari. ${ }^{74}$ Nella Tabella 5 sono sintetizzati i possibili fattori correlati all'abuso alcolico dopo il pensionamento.

\section{Attività fisica}

Un'attenzione specifica dovrebbe essere rivolta ai pensionati di bassa istruzione e agli adulti recente-

Tabella 4. Fattori coinvolti in un potenziale invecchiamento infelice. ${ }^{6}$

\section{Fattori medici}

- Sedentarietà e sindrome ipocinetica

- Alterazioni dell'equilibrio e sindrome vertiginosa

- Tendenza all'allettamento

- Abitudini voluttuarie (fumo, alcool, ecc.)

- Deficit sensoriali (vista, udito)

- Alterazione della masticazione (edentulia totale o parziale)

- Alimentazione inadeguata

- Patologia del piede

- Poli-patologia cronica invalidante tipica dell'età senile

- Alterazioni mentali e tono dell'umore

- Farmaco-dipendenza

- Danno iatrogenico non farmaco dipendente

Fattori psico-dinamici

- Apatia, disinteresse, isolamento

- Scarsa motivazione

- Personalità rigida ed autoritaria

- Scarsa propensione ai contatti sociali

- Scarsa creatività, rifiuto dei cambiamenti

- Abitudine a dipendere dagli altri

- Scarse capacità di adattamento

Fattori sociali ed economici

- Perdita del coniuge o di persona cara

- Iper-protezione familiare e scarsa abitudine ad essere autonomi

- Solitudine

- Stress familiare

- Pensionamento obbligatorio e perdita del ruolo

- Pregiudizi e stereotipi negativi sulla vecchiaia

- Difficoltà finanziarie

- Scarsa abitudine o pregiudizio ad usufruire dei servizi di supporto

- Mancanza o lontananza dei servizi assistenziali

Fattori ambientali/anagrafici

- Attività professionale precedente

- Fattori geografici/climatici/anagrafici

- Sradicamento dall'ambiente domestico

- Ospedalizzazione

- Istituzionalizzazione in casa di riposo

- Deprivazione sensoriale

- Inadeguatezza dell'alloggio

- Emigrazione

- Pericoli dell'urbanizzazione

- Comfort e sicurezza architettonica insufficienti 
mente pensionati, particolarmente se propensi ad una scarsa attività fisica e all'aumento del tempo passato a guardare la TV e all'uso del computer. ${ }^{75}$ I programmi sanitari finalizzati all'implementazione dell'attività fisica nel post-pensionamento risultano efficaci. ${ }^{76}$

\section{Fattori psicodinamici correlati ad un pensionamento infelice}

In questo ambito giocano un ruolo importante l'apatia, l'isolamento e la scarsa propensione ai contatti sociali. Uno studio con ampia casistica (campione rappresentativo di 10.513 persone in pensione provenienti da 51 paesi sottoposto a questionario) ha mostrato che l'appartenenza a più gruppi sociali ha predetto positivamente la salute e il benessere dei pensionati. ${ }^{77}$ In un altro studio longitudinale i pensionati appartenenti a 2 gruppi sociali prima del pensionamento avevano un rischio di morte del 2\% nei primi 6 anni di pensionamento, un rischio del $5 \%$ se perdevano l'adesione a un gruppo e un rischio del $12 \%$ se perdevano entrambi i gruppi. Inoltre, per ogni perdita di adesione ad un gruppo nell'anno successivo al pensionamento, la qualità di vita 6 anni dopo era inferiore di circa il 10\%. L'effetto dell'appartenenza al gruppo sociale sulla mortalità era paragonabile a quello dell'esercizio fisico. ${ }^{78}$

\section{Fattori sociali ed economici}

Diversi dati suggeriscono vari fattori di rischio socio-economico e psicosociale per i sintomi della depressione post-pensionamento possono essere rilevati già nella mezza età. Le avversità nella mezza età sono state valutate come avversità socio-economiche autoriferite: bassa posizione occupazionale, standard di vita scadente e avversità psicosociali, quali alta tensione lavorativa, poche relazioni amicali. ${ }^{79} \mathrm{Da}$ uno studio prospettico di coorte effettuato in 3.939 dipendenti pubblici britannici, che hanno risposto ad un questionario autosomministrato in età media e in età avanzata (21 anni dopo, età media 67.6 anni al follow-up), è risultato che le avversità socioeconomiche e psicosociali midlife predicono fortemente i sintomi depressivi post-pensionamento: in particolare la bassa posizione occupazionale (odds ratio [OR]: 1,70 , intervallo di confidenza al 95\% [IC]: 1,15-2,51), lo standard di vita mediocre (OR: 2,37, IC 95\%: 1,663,39 ), stress di lavoro elevato (OR: 1,52, IC 95\%: 1,09-2,14) ed i pochi rapporti stretti (OR: 1,51, $95 \%$ IC: $1.12-2.03){ }^{80}$ In realtà anche in questo ambito $\mathrm{i}$ dati sono contrastanti. Da una recente revisione sistematica che ha incluso 22 studi, 13 studi hanno rilevato effetti più positivi dell'uscita dal lavoro in salute tra i dipendenti con uno stato socioeconomico (socioeconomic status, SES), inteso come livello di istruzione, di lavoro e /o di reddito) più elevato rispetto ai dipendenti con un SES più basso. Questi effetti sono stati riscontrati principalmente a seguito di un pensionamento anticipato. In conclusione, gli effetti dell'uscita dal lavoro, o, specificamente, gli effetti del pensionamento anticipato sulla salute sono diversi tra i gruppi socioeconomici. Le politiche di sanità pubblica dovrebbero cosi concentrarsi sul miglioramento della salute dei lavoratori con uno stato socio-economico più basso, in particolare dopo l'uscita dal lavoro. Tuttavia, i risultati di questa revisione dovrebbero essere interpretati con cautela, poiché gli studi hanno utilizzato livelli di salute eterogenei e ciascun risultato di salute è stato dedotto da un numero limitato di studi inclusi. ${ }^{58}$ Tra i fattori sociali risultano importanti tutti quelli correlati, ancora una volta, ad una perdita: perdita di un caro, solitudine, pensionamento obbligatorio e perdita del ruolo, la cui espres-

Tabella 5. Possibili fattori correlati all'abuso alcolico dopo il pensionamento.

\begin{tabular}{|c|c|}
\hline Fattore in causa & Considerazioni \\
\hline $\begin{array}{l}\text { Pensionamento volontario o obbligato } \\
\text { (ad esempio, per problemi di salute } \\
\text { personali o di un coniuge o per stress } \\
\text { famigliare o per pressione } \\
\text { socio-economica) }\end{array}$ & $\begin{array}{l}\text { Fattore associato ad aumento del consumo di alcol, per mancata programmazione del } \\
\text { pensionamento, spesso reso necessario dai problemi di salute, associati, a loro volta, ad aumento } \\
\text { del consumo. I motivi personali (ad esempio, desiderare di andare in pensione, in modo tale da } \\
\text { viaggiare di più o passare più tempo con i nipoti) non correlerebbero, invece, al consumo di alcol }\end{array}$ \\
\hline Rete sociale & $\begin{array}{l}\text { In alcune occupazioni c'è una forte cultura del bere, il social network di amici prima e dopo } \\
\text { il pensionamento ha un notevole impatto }\end{array}$ \\
\hline $\begin{array}{l}\text { Coinvolgimento, soddisfazione } \\
\text { e stress lavorativo }\end{array}$ & $\begin{array}{l}\text { Lasciare ambienti di lavoro stressanti può comportare un minor consumo di alcol dopo la } \\
\text { sospensione dello stress lavorativo }\end{array}$ \\
\hline Età al momento del pensionamento & $\begin{array}{l}\text { Sembra esserci un momento ottimale per la pensione: l'abuso di alcol è probabilmente più grave } \\
\text { tra coloro che vanno in pensione in età relativamente giovane e tra coloro che continuano a lavorare } \\
\text { nonostante siano idonei al pensionamento }\end{array}$ \\
\hline Stress finanziario & Minori entrate disponibili in pensione correlano ad abuso \\
\hline Problemi coniugali & $\begin{array}{l}2 / 3 \text { dei pensionati sperimentano maggiori conflitti coniugali e minore soddisfazione coniugale nei } \\
\text { primi } 2 \text { anni di pensionamento }\end{array}$ \\
\hline
\end{tabular}


sione più rilevante è data dal lutto. Dopo una grave perdita, come la morte di un coniuge, fino a un terzo delle persone subisce effetti dannosi sulla salute fisica o mentale. Tali lutti aumentano il rischio di morte per malattie cardiache e il suicidio, oltre a causare una varietà di disturbi psicosomatici e psichiatrici. Circa un quarto delle vedove/i sperimentano depressione clinica e ansia durante il primo anno di lutto; il rischio scende a circa il $17 \%$ entro la fine del primo anno e continua a diminuire successivamente. ${ }^{81}$ In una casistica elaborata sulla base di questionari compilati dai pensionati nell'associazione depressione/pensionamento sembrano, tuttavia, di scarsa influenza la perdita di persone care, i cambiamenti del nucleo familiare ed il fatto di viver soli. ${ }^{7}$ In una casistica elaborata sulla base di questionari compilati dai pensionati un buon stato di salute precedente non sembra offrire protezione all'aumento della mortalità dopo il lutto, ma, al contrario si assiste ad un aumento di morti improvvise impreviste. ${ }^{82}$ Andrebbe quindi approfondita, con ulteriori studi, la misura in cui il pensionamento possa esporre ulteriormente al dolore diseredato. Si evidenzia, in ogni caso, la necessità di offrire un sostegno universale agli anziani ed ai pensionati al momento del lutto.

\section{Invecchiamento attivo}

Voglio ritirarmi dal lavoro.

Se ti ritiri da... è male.

Se ti ritiri per... è bene,

ma deve essere per... qualcosa di più significativo della TV!

Secondo la definizione dell'Organizzazione Mondiale della Sanità, l'invecchiamento attivo è un processo di ottimizzazione delle opportunità relative alla salute, partecipazione e sicurezza, allo scopo di migliorare la qualità della vita delle persone anziane. . $^{83,84}$ Secondo La Commissione Europea l'Europa può affrontare le sfide del cambiamento demografico solo attraverso l'invecchiamento attivo: da esso dipendono il suo futuro benessere e coesione sociale. L'invecchiamento attivo è una parte essenziale della strategia Europa 2020. Gli anziani devono poter rimanere attivi come lavoratori, consumatori, assistenti, volontari e cittadini portatori di interessi; occorre rafforzare la partecipazione della persona anziana alla vita attiva della comunità e contrastare le conseguenze negative legate a situazioni di solitudine e percezioni di inutilità sociale.$^{85}$ Tale processo, che mai dovrebbe essere considerato vessatorio, dovrebbe interessare l'intero ciclo di vita, proprio quale strumento di miglioramento esistenziale del singolo individuo. L'Unione Europea ${ }^{86}$ indica quattro aree principali nelle quali intervenire: i) l'ambito lavorativo: ovvero promuovere l'invec- chiamento attivo sul luogo di lavoro; ${ }^{87}$ ii) la partecipazione sociale; iii) la salute ed il mantenimento dell'autonomia personale; iv) la solidarietà tra le generazioni. Molteplici sono i modi attraverso i quali è possibile promuovere la partecipazione sociale degli anziani (ad esempio: attività di volontariato, cittadinanza attiva, sviluppo di reti sociali) e diverse opportunità sono offerte dal volontariato, come il network SEVEN, ${ }^{88}$ una rete europea di 29 organizzazioni che promuovono l'apprendimento continuo e il volontariato tra le persone anziane. In uno studio effettuato nel Regno Unito, negli USA e in Italia su 133 soggetti, condotto 10 mesi prima del pensionamento è stato chiesto quali fossero i progetti per il futuro, per approfondire la corrispondenza con gli indici d' invecchiamento attivo (Active Aging Index): il mantenimento di un lavoro dopo la pensione, la partecipazione alla vita sociale e famigliare, il grado d'indipendenza economica, l'autonomia psico-fisica (comprendente salute, attività motoria e studio) e la capacità di mantenere relazioni con amici, parenti e colleghi. I risultati suggeriscono che, in linea di massima, la pianificazione del ritiro dall'attività lavorativa è sostanzialmente coerente con la prospettiva dell'invecchiamento attivo, anche se tra i parametri da considerare dovrebbero essere aggiunte tutte quelle attività che attengono al tempo libero, ovvero di tutte quelle attività dedicate allo svago, il cosiddetto active leisure. Diverse Regioni hanno organizzato per le persone anziane un coinvolgimento in varie attività quali: animazione, gestione, custodia e vigilanza di musei, biblioteche e parchi pubblici, vigilanza sul patrimonio monumentale ed architettonico delle città, conduzione di appezzamenti di terreno e orti, implementazione delle tradizioni, dei mestieri e dell'artigianato locale, assistenza culturale e sociale negli ospedali e nelle carceri, interventi di carattere ecologico nel territorio, eccetera. Nella pianificazione desiderata s'inserisce però un serio problema intercorrente, quello dell'obbligo di accudire i familiari, specialmente anziani, che - almeno nel campione italiano - deriva da una scelta affettiva nel contesto famigliare. La volontà di mantenere un'attività lavorativa dopo la pensione è diverso nei vari paesi: non rientra tra le preferenze degli italiani (anche in presenza di difficoltà economiche), tra gli inglesi prevale l'opzione di mantenersi attivi senza obblighi fissi (ad esempio con un impegno un giorno a settimana, per sei mesi all'anno, onde poter negli altri sei mesi, poter viaggiare), i nord-statunitensi vorrebbero essere impegnati in qualcosa di diverso, con un impiego che possa modificare lo stile di vita. La mancata pianificazione delle attività future comporta in alcuni casi uno stato d'ansia che può compromettere le sensazioni positive generalmente associate al pensionamento. È necessario quindi un maggiore supporto durante la transizione lavoro-pen- 
sione, mediante corsi o colloqui di orientamento. Secondo le opinioni degli intervistati, le aziende potrebbero fornire maggiore supporto durante la transizione al pensionamento, con opportunità di mantenere una connessione sociale con ex colleghi e contribuire a realizzare e adempiere ai piani pensionistici. ${ }^{89}$ Vanno considerate, infine le possibili conseguenze di conflitti intergenerazionali legate all' 'implementazione dell'invecchiamento attivo e la conseguente opportunità d'impegnarsi per una maggior coesione sociale. ${ }^{90}$

\section{Come prevenire gli effetti negativi della cessazione dell'attività lavorativa: consigli per un pensionamento felice e di successo}

\author{
...La giovinezza è il tempo \\ per studiare la saggezza; \\ la vecchiaia è il tempo di metterla in pratica ${ }^{91}$
}

L'invecchiamento non è una gioventù persa $m a$ una nuova fase di opportunità e di rinnovata forza $\mathrm{e}$ l'unico modo per una donna, come per un uomo, di ritrovare se stessa, di conoscersi come persona, è il suo lavoro creativo..$^{92} \mathrm{La}$ vecchiaia è l'età del tempo libero e bisogna adoperarsi per non renderlo un tempo vuoto, di fatto corrispondente ad una morte precoce. ${ }^{93}$ Alcune raccomandazioni potrebbero essere utili ai fini della pianificazione futura per le persone in una società che invecchia per evitar un invecchiamento precario, in termini sia di felicità che di salute ${ }^{94}$ (vedi paragrafo successivo).

\section{Possibili utili indicazioni per un pensionamento felice}

- Sul piano programmatico sociale: quali garanzie dovrebbero essere dovute? i) Implementare politiche di invecchiamento attivo, con il coinvolgimento degli enti locali (i comuni in prima linea) e garantendo che le persone anziane siano impegnate in modo produttivo (retribuito o volontario) nella società, con incentivi adeguati, sia per i datori di lavoro che per i singoli individui; ii) favorire l'anticipazione del pensionamento per i lavoratori addetti a lavori usuranti e a turni notturni e procrastinarlo per coloro che, in buona salute, vogliono continuare a lavorare; iii) incentivare politiche di pensionamento flessibile e/o progressivo, secondo scelte soggettive e in funzione della compatibilità economica, favorendo il part-time; iv) incoraggiare la sicurezza economica individuale e sociale, con garanzie per i risparmi individuali e fornendo un'assistenza sanitaria pubblica di alta qualità a tutti, compreso il rafforzamento dell'assistenza dedicata agli anziani e supporti ade- guati per i care giver; v) rafforzare, con politiche ad hoc, il patto sociale tra anziani e giovani, nella società e nel contesto famigliare, allo scopo di garantire un giusto riequilibrio intergenerazionale.

- Sul piano soggettivo: pianificare in anticipo e perseguire obiettivi specifici per sè stessi: i) non ritirarsi dal contatto quotidiano con famigliari e con amici e colleghi; ii) evitando il più possibile conflitti e cercando di risolvere le controversie; ovvero: coltivare gli affetti e le relazioni famigliari e sociali, mettendo a disposizione il proprio impegno, ricercando mediazioni e soluzioni costruttive e positive; iii) nel contesto famigliare ridefinire gli intenti allo scopo di rinegoziare/riorganizzare la vita insieme: il più delle volte il momento della pensione non coincide tra i due, dando origine ad ulteriori criticità; iv) mantenere il controllo della propria vita ed un ruolo sociale, in funzione delle proprie competenze e la propria creatività, con nuove idee, accettando le sfide per nuove esperienze, professionali e non; v) mantenere, particolarmente nel tempo libero, le attività già praticate o svilupparne di nuove, quali: attività sportive e/o ludiche, giardinaggio, hobby, arte, lettura, scrittura, cultura, cucina, viaggi, cinema, teatro, pratiche ginniche o meditative (mindfulness), per mantenere una vita quotidiana attiva e propositiva; $95 \mathrm{vi}$ ) ricercare sistematicamente piacere, sollievo, curiosità, gioia di dare, per evitare il senso di vuoto e la sensazione che il mondo vada avanti senza di noi, ${ }^{96}$ secondo le proprie attitudini, per conservare le abilità cognitive e per affrontare i cambiamenti fisici, sociali ed emotivi, ovvero: a) avere sempre curiosità nuove e restare sempre attivi (ad esempio, iscrivendosi ad un'Università della terza età), con studio, lettura, pittura, fotografia, giardinaggio, nordik walking, ${ }^{97}$ Tai Chi ${ }^{98}$ (e altre forme di movimento; ${ }^{99} \mathrm{~b}$ ) migliorare la work ability dei lavoratori (anziani e non) mediante interventi di promozione della salute (soprattutto su abitudine al fumo e attività fisica), per prevenire le disabilità, le cadute e le malattie da scarso movimento (ex non usu), garantirsi un'adeguata alimentazione, senza esagerare (mangia e bevi per la tua salute; stai attento al 'girovita' ma anche 'girati la vita'se questa non ti soddisfa); c) garantirsi un'affettività personale con emozioni positive ed interpersonali gratificanti: amare, ridere, gioire, godere, anche ridimensionando le proprie aspettative per non crearsi disillusioni, magari anche allargando la sfera affettiva ad animali domestici, ai fini di un'utile pet therapy; d) arricchire il lato spirituale della propria vita, perseguendo le proprie idee religiose e le proprie vocazioni, aiutando altre persone a facilitare il loro futuro; l'impegno nel volontariato, con messa a disposizione delle 
proprie capacità individuali per gli altri costituisce un ottimo strumento per rendersi utili nella società e confermare il proprio sé; e) prevenire il decadimento cognitivo, ${ }^{100}$ anche con metodi moderni: il NeuroRacer, è un videogioco progettato da un team di ricercatori dell'Università di San Franci$\mathrm{sco})^{101}$ che ha dimostrato essere utile per migliorare la cognizione mentale e la memoria; f) continuare ad apprendere (aun aprendo), esplorando nuovi campi di interesse da cui siamo sempre stati incuriositi. Rispondi alla domanda: $\cos a$ avrei voluto fare se non avessi fatto ... il mio lavoro?: in sostanza crea per confermare la tua creatività!!

\section{Conclusioni}

Aun aprendo (1826) - (imparo ancora), a 92 anni!

Francisco de Goya (1746-1828) - Madrid, Museo del Prado

Enzo Bianchi nel suo ultimo libro, a proposito delle barbe bianche degli anziani ${ }^{102}$ ci spiega che nel Vecchio Testamento, in ebraico, il vecchio viene definito zaqen, che sta proprio per barba, perché la vecchiaia era l'età in cui non ci si radeva più e che un altro termine, impiegato per indicare la senescenza, seib-seiba, evocava l'incanutimento quale indizio di «una beata e serena stagione della vita, quella in cui gustare la pienezza dei giorni»» ${ }^{103}$ Gli anni del pensionamento si trascorrono più o meno serenamente sulla base di quello che si è stati prima e per prima s'intende durante tutta la vita ${ }^{104} \mathrm{Il}$ pensionamento è l'interruzione della propria attività lavorativa o professionale, non un cambiamento nel proprio stile di vita. L'epoca del pensionamento è una soglia artificiale di cambiamento esistenziale, imposta dalla società ad una determinata età, che può portare, in coloro che non si sono preparati in precedenza, ad una sindrome da disadattamento, con possibili conseguenze per la salute ed aumentato rischio di invecchiamento infelice e/o patologico. In altri soggetti, invece, il pensionamento viene visto come liberazione da un peso lavorativo oramai insopportabile. Spetterebbe al mondo del lavoro e alle politiche sociali combattere una cultura dell'emarginazione, che non facilita il cambiamento e che fa coincidere la pensione come tappa obbligata di passaggio dall'essere membro attivo della società che genera e produce, all'esserne ospite inoperoso e passivo, in cui prevale il disimpegno, il ritiro, il ripiegamento su di sé. ${ }^{105}$ Alla luce delle correlazioni tra invecchiamento, pensionamento e stato di salute è lecito chiedersi se le politiche pensionistiche $\mathrm{e}$, in particolare, se il pensionamento obbligatorio ope legis sia ancora uno strumento indispensabile. In alcuni Paesi (ad esempio, negli USA) non esiste un'età defi- nita dalla legge come obbligata per andare in pensione. L'innalzamento - se non la liberalizzazione - oltre un'età limite minima - dell'età pensionabile dovuta a motivazioni economiche, rappresenta uno degli strumenti oggi disponibili per affrontare la sfida posta dall'invecchiamento della popolazione, in uno scenario in cui il giovanilismo e l'ageismo mediatico imperano e la sostenibilità finanziaria è sempre più precaria. Oggi in Italia abbiamo bisogno di flessibilità, con l'introduzione di aggiustamenti sostenibili per un equo accesso alla pensione. ${ }^{1}$ Serve una visione di futuro, in un progetto per le pensioni che non si rivolga solamente indietro. ${ }^{106} \mathrm{Si}$ dovrebbe considerare la possibilità che, una volta raggiunta l'età anagrafica pensionabile, l'attività lavorativa retribuita possa continuare volontaristicamente e sempre in accordo con il datore di lavoro. Come internisti non vogliamo entrare più di tanto nel dibattito tecnico-politico sul tema delle riforme pensionistiche necessarie nel nostro Paese, spesso gravato da motivazioni meramente ideologiche, se non demagogiche. Riteniamo che l'estensione dell'attività lavorativa, secondo una scelta soggettiva ad libitum e quoad valetudinem, con un impegno fattivo, attivo e proattivo delle persone, quando possibile, sia una scelta di vita assolutamente imprescindibile, forse migliore di qualsiasi terapia farmacologica, ai fini della prevenzione primaria e secondaria di molte malattie. Lavorare più a lungo può essere utile per la prevenzione di un invecchiamento patologico ed il benessere soggettivo degli anziani. Vanno verificati i possibili ridotti aggravi a carico della spesa sanitaria pubblica (sempre che potrà esistere un welfare state ed un Servizio Sanitario Nazionale in futuro nel nostro Paese!). Ci sentiamo però di prescrivere una ricetta molto economica per la società, ma molto sfidante ed impegnativa per le singole persone: mantenersi in attività il più possibile, mostrare curiosità ed energia vitale, partecipazione, disponibilità al cambiamento, avere comportamenti alimentari e voluttuari adeguati, un movimento attivo, voglia di apprendere e capacità di autogestire le proprie malattie croniche. Su questo tema le Società Scientifiche mediche ed infermieristiche come la FADOI e l'ANIMMO possono fattivamente contribuire per progetti di ricerca finalizzata, allo scopo di approfondire ulteriormente gli effetti della cessazione dell'attività lavorativa sullo stato di salute, nonché a progetti educazionali rivolti ai pazienti e ai care giver sul self management delle malattie croniche.

\section{Messaggi chiave}

i) I dati epidemiologici mostrano lo scenario futuro di una popolazione che invecchia. Il pensionamento, evento ad elevato impatto nell'esistenza di una persona, non è un evento subitaneo, ma si svolge attraverso fasi progressive, cui dovrebbe corrispondere un adattamento. 
ii) Ciascuno di noi dovrebbe essere consapevole dell'opportunità di sforzarsi sempre, in ogni momento della vita, di raggiungere un equilibrio tra lavoro e tempo libero e delle implicazioni correlate alla cessazione dell'attività lavorativa.

iii) L'adattamento al pensionamento risulta migliore se esiste una pianificazione precedente, mediata tra attività lavorativa, famiglia e tempo libero; l'interruzione brusca dell'attività lavorativa può in alcuni casi comportare uno shock da pensionamento, particolarmente in alcune condizioni di rischio. In sostanza bisognerebbe reinventarsi per le nuove sfide poste dalla cessazione dell'attività lavorativa.

iv) Un rallentamento della velocità di transizione tra attività lavorativa e pensionamento può facilitare l'adattamento del singolo individuo.

v) Diversi fattori sono correlati ad un pensionamento infelice, di tipo medico, psico-dinamico, sociale ed economico. L'adattamento al pensionamento è migliore se intervengono comportamenti proattivi (attinenti le abitudini di vita, l'ambito lavorativo; la partecipazione sociale; la salute e l'autonomia personale; la solidarietà tra le generazioni),

vi) La flessibilità di un pensionamento progressivo potrebbe consentire al singolo individuo una maggior libera scelta su quando ritirarsi dal lavoro, anche per consentire un disimpegno graduale dall'attività professionale.

vii) Le riforme a favore di un pensionamento tardivo volontario (auspicabili soprattutto per i lavori non usuranti e negli anziani in buone condizioni di salute), non sono importanti solo per una migliore solvibilità dei sistemi pensionistici, ma anche, nel tempo, per migliorare la qualità di vita delle persone, riducendo i costi dell'assistenza sanitaria pubblica.

viii) L'ipotesi dell'utilità di una solidarietà espansiva determinata dalla staffetta generazionale non è così certa né documentata e non vi è certezza di ricambio automatico (sia nel settore pubblico che privato) tra posti di lavoro lasciati liberi dagli anziani e conseguente ricambio da parte di lavoratori giovani.

ix) È necessario migliorare le relazioni inter-generazionali e l'integrazione del sapere e del fare tra vecchi e giovani: i primi custodi dell'esperienza, i secondi portatori di entusiasmo e di capacità di modernizzazione/innovazione).

x) L'educazione all'invecchiamento (geragogia) fin dall'età adulta giovanile e alla capacità di autogestire le malattie croniche è fondamentale sempre, e particolarmente nelle ultime fasi dell'attività lavorativa. Le Società Scientifiche medico-infermieristiche possono avere in questo un ruolo determinante.

\section{Bibliografia}

1. Brambilla A, Un sistema che ha bisogno di flessibilità, in AA. Vari, Pensionare la riforma Fornero? A cura di Impresa Lavoro-Centro Studi, Suppl. a "Il Giornale", 2018, pp. $75-79$.

2. ISTAT, Indicatori demografici, https://www.istat.it/it/archivio/18049

3. The Quest for Lasting Stability -- IMF Global Financial Stability Report (GFSR) -- April 2012 -- Table of Contents. At: http://www.imf.org/External/Pubs/FT/GFSR/ 2012/01/index.htm

4. Nardi R, Mathieu G, La Regina M, Profilo biosociale del paziente ospedalizzato del XXI secolo, Quad Ital J Med 2018, 6/3: 4-8

5. Sgroi, M, La triste vecchiaia in Europa, 21 gennaio 2014, https://formiche.net/2014/01/la-triste-vecchiaiadelleuropa/

6. Rosina A, La crescita demografica perduta, https:// www.lavoce.info/archives/40477/la-crescita-perduta-passato-presente-e-futuro-della-popolazione-italiana/, 05.04.16

7. Nardi R, Corneli M, Marchetti C, Calogero P, Cavazzuti F, Pensionamento: ritirarsi dal lavoro non dalla vita. Aspetti medici e psicologici, Coop Editoriale Nuova Brianza, Renate (MI), 1987

8. Wang M, The Oxford Handbook of Retirement, OUP USA, 8 nov 2012

9. Talini D, Ghersi R, Gruppo Invecchiamento e Lavoro CIIP - Consulta Inter-associativa Italiana per la Prevenzione, AGING e-Book di CIIP: il libro d'Argento su Invecchiamento e Lavoro http://www.ciip-consulta.it/, https://www.ciip-consulta.it/images/eventlist/Eventi 2018/180511-Roma/Talini_11-5-18.pdf)

10. Atchley RC, Retirement and leisure partecipation: continuity or crisis? Gerontologist 1971, 2/1, 13

11. Kübler-Ross E, On death and dying, Macmillan, 1969. Riedito come On Death and Dying. What the Dying Have to Teach Doctors, Nurses, Clergy and Their Own Families, Taylor \& Francis, $40^{\mathrm{a}}$ ed. 2008. ISBN 0-41546399-8; ISBN 978-0-415-46399-7. Anteprima limitata. Trad. it.: La morte e il morire, Assisi, Cittadella, 1976. $13^{\mathrm{a}}$ ed.: 2005. ISBN 88-308-0247-6; ISBN 978-88-3080247-6

12. Santucci U. Cambiamento: il modello di Kübler-Ross. Disponibile a: http://www.umbertosantucci.it/atlante/cambiamento-il-modello-di-kubler-ross/

13. Cristini C, Rizzi R, Zago S, La vecchiaia fra salute e malattia, Pendragon Ed, in: Collana: Sentieri della mente, 2005

14. Antonini FM, Fumagalli C, Gerontologia e Geriatria, Wasserman Ed, Milano 1976

15. Peretti O, Wilson C, Voluntary and Involuntary Retirement of Aged Males and their Effect on Emotional Satisfaction, Usefulness, Self-Image, Emotional Stability, and Interpersonal Relationships, The International Journal of Aging and Human Development, 1975, 6/2: 131-138

16. Jokela $\mathrm{M}$ et al, From Midlife to Early Old Age: Health Trajectories Associated With Retirement, Epidemiology 2010, 21/3: 284-290

17. Villamil E, Huppert F, MelzerD, Low prevalence of depression and anxiety is linked to statutory retirement 
ages rather than personal work exit: a national survey, Psychological Medicine, 2006, 36: 999-1009

18. Westerlund H, Vahtera J, Ferrie JE et al, Effect of retirement on major chronic conditions and fatigue: French GAZEL occupational cohort study, BMJ 2010; 341: c6149

19. Behncke S, How Does Retirement Affect Health?, Discussion Paper No. 4253 June 2009 IZA, http://ftp.iza. org/dp4253.pdf

20. Kimmel DC, Price KF, Walker JW, Retirement Choice and Retirement Satisfaction, Journal of Gerontology 1978, 33/4: 575-585

21. Atherley-TC, The Effect of Statutory Retirement on Healthy Ageing, https://pdfs.semanticscholar.org/presentation/0613/7b2d50f40a1247f4c7946bf7162f75e145 8c.pdf

22. Voltaire-Francois-Marie Arouet, Candide, ou l'Optimisme, 1759, Candido, ovvero l'ottimismo, traduzione di Tommaso Maria Grandi, Milano, BCDalai, 2009

23. Gilliand P, Ageing of the population and employment of the elderly, Council of Europe: Population and vocational training division, Strasbourg, 10 oct 1974

24. Lundberg GD, Retirement May Be Hazardous to Your Health?, Medscape J Med 2008; 10/5: 112

25. Lundberg GD, What Do You Think About Retirement?, https://www.medscape.com/viewarticle/904959?nlid=1 26573_2049\&src $=$ WNL_mdplsnews_181214_mscpedit_imed\&uac $=64127 \mathrm{CN} \&$ spon $=18 \& \overline{\mathrm{imp}} \mathrm{ID}=1 \overline{8} 3147$ $7 \& \mathrm{faf}=1$ December 07,2018

26. Boeri T, Per un invecchiamento attivo, Convegno AIP su «Le nuove tecnologie e il valore sociale della ricerca psicologica», Relazione presentata a Roma, 19 settembre 2016

27. D'Errico A, Lavoro e invecchiamento: Condizioni di lavoro e di salute dei lavoratori anziani, Camera del Lavoro di Torino, 21 ottobre 2015

28. Bamia C, Trichopoulou A, Trichopoulos D. Age at retirement and mortality in a general population sample: the Greek EPIC study. Am J Epidemiol. 2008;167:561-569

29. Bonsang E, Adam S, Perelman S, Does retirement affect cognitive functioning? J Health Econ. 2012, 31/3: 490-501

30. Xue B, Cadar D, Fleischmann M et al, Effect of retirement on cognitive function: the Whitehall II cohort study, Eur J Epidemiol 2018, 33/10: 989-1001

31. de Zwart BCH et al, Physical workload and the ageing worker: A review of the literature, International Archives of Occupational and Environmental Health 1995; 68/1: 1-12

32. de Zwart BCH et al Musculoskeletal complaints in The Netherlands in relation to age, gender and physically demanding work, International Archives of Occupational and Environmental Health 1997, 70/5: 352-360

33. Ilmarinen J, Physical requirements associated with the work of aging workers in the European Union. Experimental Aging Research 2002, 28/1: 7-23

34. Stenholm S, Westerlund H, Salo P et al, Age-related trajectories of physical functioning in work and retirement: the role of sociodemographic factors, lifestyle and disease, J Epidemiol Community Health 2014, 68: 503-509

35. Maimaris W, Hogan H, Lock K. The impact of working beyond traditional retirement ages on mental health: Implications for public health and welfare policy, Public Health Reviews 2010; 32: 532-548
36. Belloni M, Meschi E, Pasini G, The effect on mental health of retiring during the economic crisis, Health Economics 2016, 25/S2: 126-140

37. Di Gessa G, Corna L, Price D, Glaser K, The decision to work after state pension age and how it affects quality of life: evidence from a 6-year English panel study, Age and Ageing 2018, 47/3: 450-457

38. Kachan D, Fleming LE, Christ S et al, Health Status Of Older Us Workers And Non workers, National Health Interview Survey, 1997-2011, Prev Chronic Dis 2015,150040. DOI: http://dx.doi.org/10.5888/pcd12. 150040

39. Tomioka K, Beneficial effects of working later in life on the health of community-dwelling older adults, Geriatr Gerontol Int 2018, 18: 308-314

40. Heller-Sahlgren G, Retirement blues, J Health Econ 2017, 54: 66-67

41. Pica F, Quando è possibile trattenersi in servizio dopo l'età pensionabile, https://www.pensionioggi.it/notizie/lavoro/pensioni-quando-e-possibile-trattenersi-in-serviziodopo-1-eta-pensionabile-6545646\#ixzz5RXpI7vIU

42. Ricolfi L, Pensione e lavoro: troppi stereotipi, poca conoscenza, Panorama 13 novembre 2017

43. Santoro E, Moriconi S, Non saranno i prepensionamenti a ridare lavoro ai giovani, https:/www.linkiesta.it/it/article/2016/04/26/non-saranno-i-prepensionamenti-a-ridare-lavoro-ai-giovani/30118/, 26 aprile 2016

44. Patriarca S, Prepensionare per fare lavorare i giovani è una truffa, Il Foglio 23 settembre 2018, https://www.ilfoglio.it/economia/2018/09/23/news/prepensionare-perfare-lavorare-i-giovani-e-una-truffa-214900/

45. OECD, Pensions at Glance 2017, https://read.oecd-ilibrary.org/social-issues-migration-health/pensions-at-aglance-2017 pension glance-2017-en\#page2

46. Sensini M, Corriere della Sera Economia, 19 aprile 2016modifica il 20 aprile 2016, https://www.corriere.it/economia/16_aprile_19/inps-lancia-1-allarme-classe-1980-risch ia-pensionamento-75-anni-116f6e94-0657-11e6-98add281ab178a74.shtml?refresh_ce-cp

47. De Rita G, Da vecchi aumenta la voglia di lavorare, Il Medico d'Italia, 1985, 40: 4

48. Sahlgren GH, Work Longer, Live Healthier: The relationship between economic activity, health and government policy, IEA Discussion Paper No. 46, May 2013, https://www.iea.org.uk/sites/default/files/publications/fil es/Work\%20Longer,\%20Live_Healthier.pdf

49. Fisher GG, Chaffee DS, Sonnega A, Retirement Timing: A Review and Recommendations for Future Research. Work, Aging and Retirement 2016, 2: 230-261

50. Special Eurobarometer 378, Active Ageing, Active Ageing, Summary, January 2012, http://ec.europa.eu/ commfrontoffice/publicopinion/archives/ebs/ebs_378_s um_en.pdf

51. Geoldi G, Gli oneri del pensionamento flessibile, http://nuovo.lavorowelfare.it/wp-content/uploads/ 2015/12/2015-10-27-G2-pensionamento-flessibile.pdf

52. Nardi R, La necessità di prepararsi al pensionamento, Giorn Gerontol 1982, 30: 52

53. Chen YP, Scott Jc, Il pensionamento progressivo negli USA: chi lo sceglie e a che scopo, Quaderni Europei del novo welfare, 7, Feb. 2007, Verso il nuovo welfare: obiettivi e strategie, http://www.newwelfare.org/2007/02/ 26/quaderno-n7-febbraio-2007-elenco-contenuti/ 
54. Brambilla A, Corriere L'Economia del 9/4/2018.

55. Redazione Wall Street Italia, Pensione ormai un miraggio, http://www.wallstreetitalia.com/pensione-ormai-unmiraggio-in-usa-e-non-solo-si-lavorer-fino-a-80-anni/

56. Macis V, Come prepararti alla pensione negli Stati Uniti? (https://italiandistrict.com/florida/consulenza/sistema-pensioni-usa/

57. VanDerher J, EBRI Retirement Security Research Program, https://www.ebri.org/about/team/index.cfm?fa= JackVanDerhei; Maggi G, Negli Usa un futuro di pensioni da fame e vecchi al lavoro. Anche a 80 anni difficile avere un assegno decente", http:/www.lastampa.it/2011/06/ 20/economia/negli-usa-un-futuro-di-pensionida-fame-evecchi-al-lavoro-pAYb1kYSJx5Vyx5nIZkpqJ/pagina .html

58. Fornero E, Monticone C. Il pensionamento flessibile in Europa, http://www.cerp.carloalberto.org/wp-content/ uploads/2008/12/forneromonticone pensionamentoflessibile.pdf; European Commission (2006a), Social protection in the Member States of the European Union, of the European Economic Area and in Switzerland Comparative Tables, Brussels. European Commission (2006b), Synthesis report on adequate and sustainable pensions. Horizontal analysis, Brussels

59. Brambilla A, Pensioni flessibili per una popolazione che invecchia, http://www.ilpuntopensionielavoro.it/site/ home/il-punto-di-vista/pensioni-flessibili-per-una-popolazione-che-invecchia.html)

60. Valenti V, Diritto alla pensione e questione intergenerazionale: Modello costituzionale e decisioni politiche, G Giappichelli Ed, Torino, 2013

61. Grignoli D, L'apprendimento intergenerazionale nei sistemi lavorativi: un possibile percorso di coesione, Scienze e Ricerche, 18 febbraio 2015, http://www. scienzericerche.it $/ \mathrm{p}=2657$

62. Bossio F, Formazione e quarta età. Prospettive pedagogiche, 2002, Filena, Napoli

63. Melton B, Life's Transitions, The Aging Process, 2010, https://slideplayer.com/slide/11910767/

64. Schaap R, de Wind A, Coenen P, Proper K, Boot C, The effects of exit from work on health across different socioeconomic groups: A systematic literature review, Soc Sci Med. 2018; 198: 36-45

65. Chermack ST, Blow FC, Hill EM, Mudd Sam, The relationship between alcohol symptoms and consumption among older drinkers, Alcohol Clin Exp Res.1996; 20: $1153-1158$

66. American Geriatric Society. Clinical guidelines for alcohol use disorders in older adults; 2003. http://www. americangeriatrics.org/products/positionpapers/alcohol.shtml

67. Yogev S, A Couple's Guide to Happy Retirement and Aging 15 Keys to a Lasting Relationship, March 1, 2018

68. Emiliussen J, Andersen K, Nielsen AS, Why do some older adults start drinking excessively late in life? Results from an Interpretative Phenomenological Study. Scand J Caring Sci 2017; 31: 974-983

69. Botman SL, Moore TF, Moriarity CL, et al. Design and estimation for the National Health Interview Survey, 1995-2004, https://www.cdc.gov/nchs/data/series/sr_02/ sr02_130.pdf

70. Rudolf H. Moos, Kathleen K. Schutte, Penny L. Older Adults' Alcohol Consumption and Late-Life Drinking
Problems: A 20-Year Perspective. Addiction. $2009 \mathrm{Au}-$ gust ; 104(8): 1293-1302 NIH Public Access on line su: https://www.ncbi.nlm.nih.gov/pmc/articles/PMC271487 3/pdf/nihms

71. Kuerbis, A. and Sacco, P. The impact of retirement on the drinking patterns of older adults: A review. Addictive Behaviours, 2012. 37 (5): 587-595

72. Zantinge, E., Van den Berg, M., Smit, H., and Picavet, H. Retirement and a healthy lifestyle: opportunity or pitfall? A narrative review of the literature. European Journal of Public Health 2014, 24 (3): 433-439

73. Bamberger, P. Winding Down and Boozing Up: The Complex Link Between Retirement and Alcohol Misuse. Work, Ageing and Retirement. 2014:1-20

74. Yogev S, A Couple's Guide to Happy Retirement 2015, online: https://www.nextavenue.org/retirement-alcoholabuse

75. Van Dyck D, Cardon G, De Bourdeaudhuij I, Longitudinal changes in physical activity and sedentary time in adults around retirement age: what is the moderating role of retirement status, gender and educational level? BMC Public Health. 2016; 16: 1125

76. Lima KC, Caldas CP, Veras RP, Correa RF, Health Promotion and Education: A Study of the Effectiveness of Programs Focusing on the Aging Process. Int J Health Serv. 2017, 47/3: 550-570

77. Lam BCP, Haslam C, Haslam SA, Multiple social groups support adjustment to retirement across cultures, Soc Sci Med. 2018; 208: 200-208

78. Steffens NK, Cruwys T, Haslam C, Social group memberships in retirement are associated with reduced risk of premature death: evidence from a longitudinal cohort study, BMJ Open 2016 2016; 6(2): e010164

79. Kasen S., Chen H., Sneed J.R. Earlier stress exposure and subsequent major depression in aging women. Int $\mathrm{J}$ Geriatr Psychiatry. 2010; 25: 91-99

80. Virtanen M, Ferrie JE, Batty GD, Socioeconomic and Psychosocial Adversity in Midlife and Depressive Symptoms Post Retirement: A 21-year Follow-up of the Whitehall II Study, Am J Geriatr Psychiatry 2015, 23/1: 99-109

81. Parkes CM, Bereavement in adult life, The BMJ, https://www.bmj.com/content/316/7134/856.1, 1998

82. Shah SM, Carey IM, Harris T et al, Do good health and material circumstances protect older people from the increased risk of death after bereavement?, Am J Epidemiol. 2012, 176/8: 689-698

83. WHO, The Political Declaration and Madrid Intenational Plan of Action on ageing, 2002; The World Health Organization's Active ageing: a policy framework, 2002

84. Davidde M, L'invecchiamento attivo, Master Scienziati in Azienda, XVI edizione 2015/2016, http://www.medicinanarrativa.eu/wp-content/uploads/2016/11/Invecchiamento-attivo-di-Marianna-Davidde.pdf

85. Nardin E, Le sfide di un'utenza in continua evoluzione, SENIORnet, Venezia Mestre 8/4/2014

86. European Commission, Committee of the Regions, AGE platform Europe-EU support to local and regional actors, How to promote active ageing in Europe - (2011

87. lmarin J, Promuovere l'invecchiamento attivo sul luogo di lavoro, https://osha.europa.eu/it/node/6776/file_view

88. Senior European Volunteers Exchange Network) (http://www.programsforelderly.com/contributing-senior-volunteer-exchange-network-seven.php 
89. Principi A, Santini S, Socci M, Smeaton D, Cahill K, Vegeris S, Barnes H, Retirement plans and active ageing: Perspectives in three countries, Ageing and Society 2018, 38/1: 56-82

90. Rospi M, L'invecchiamento attivo della popolazione all'interno della coesione sociale tra generazioni: gli strumenti della multilevel governance per nuovi sistemi di welfare, Rivista AIC n: 3/2018 data pubblicazione: 02/07/2018

91. Jean-Jacques Rousseau, Le passeggiate del sognatore solitario, trad. a cura di B. Segaste, Feltrinelli Ed, 2016)

92. Betty Friedan, https://le-citazioni.it/autori/bettyfriedan/

93. Bobbio N, L'età del tempo libero, in: G. Urbani, N. Bobbio, G. Capuani e G et Al, L'anziano attivo. Proposte e riflessioni per la terza e la quarta età, Edizioni della Fondazione G. Agnelli,Torino, 1991, p. 11)

94. Rowe JW, Challenges and Opportunities for Development of a Successfully Aging Society, https://www. macfound.org/networks/research-network-on-an-aging-socie ty/details/

95. Mace C, Mindfulness and mental health. New York, Routledge, 2008. Trad. it. "Mindfulness e salute mentale". Roma, Ubaldini, 2010; Medina J, Il cervello non ha età, Nuovi Saggi Bollati Boringhieri, 2018

96. Cicerone PE, 1 vademecum del pensionato felice, http://www.lescienze.it/news/2010/10/26/news/il_vade mecum_del_pensionato_felice-554256/)

97. Bullo V, Gobbo S, Vendramin B et Al, Nordic Walking Can Be Incorporated in the Exercise Prescription to Increase Aerobic Capacity, Strength, and Quality of Life for Elderly: A Systematic Review and Meta-Analysis, Rejuvenation Research 2018 21/2, 141-161

98. Ho TJ, Ho LI, Hsueh KW et Al, Tai Chi Intervention
Increases Progenitor CD34+ Cells in Young Adults, Cell Transplant 2014, 23/4-5: 613-620

99. Li F, Harmer P, Fitzgerald $\mathrm{K}$ et al, Effectiveness of a Therapeutic Tai Ji Quan Intervention vs a Multimodal Exercise Intervention to Prevent Falls Among Older Adults at High Risk of Falling: A Randomized Clinical Trial, JAMA 2018, 1; 178/10: 1301-1310

100. Fratiglioni L, Wang HX, Brain reserve hypothesis in dementia, J Alzheimers Dis 2007; 12: 11-22

101. Anguera JA, Boccanfuso J, Rintoul JL et al, Video game training enhances cognitive control in older adults, Nature 2013, 501; 97-101

102. Bianchi E, La vita e i giorni. Sulla vecchiaia, Il Mulino Ed, Collana Intersezioni, 2018

103. Polito A, Barbe bianche, uniamoci contro il giovanilismo, 7-Corriere della Sera, 6 luglio 2018, pp 76-77, https://www.corriere.it/sette/18_luglio_05/barbebianche-uniamoci-contro-giovanilismo-5df187787e02-11e8-98cc-f2df688ea5aa.shtml?refresh_ce-cp

104. Bosio M, Educazione gerontologica: educazione alla qualità della vita, in Cavazzuti F, Gerontologia e Geriatria per infermieri, terapisti della riabilitazione e operatori socio - sanitari, Udine, Ed. COLLEGIO IPASVI, 1985, p. 545

105. Freddo S, Dal lavoro al pensionamento. vissuti ed esperienze, Tesi di Dottorato, Università degli Studi di Milano-Bicocca, Coordinatore: Prof.ssa Ottavia Albanese; Tutor: Prof.ssa Silvia Kanizsa, https://boa.unimib.it/retrieve/handle/10281/46120/68926/Tesi\%20dottorato\%2 0S.\%20Freddo-Dal\%20lavoro\%20al\%20pensionamento.\%20Vissuti\%20ed\%20esperienze.pdf

106. De Rita G, Un nodo ingarbugliato, in: AA. Vari, Pensionare la riforma Fornero?. A cura di Impresa LavoroCentro Studi, Suppl. a "Il Giornale", 2018, pp. 51-57. 


\title{
Gestione della depressione, inevitabile compagna del paziente anziano
}

\author{
Emanuela Pecoraro, ${ }^{1}$ Salvatore La Carrubba ${ }^{2}$ \\ ${ }^{1}$ Geriatria, Policlinico Universitario, Palermo; ${ }^{2}$ Medicina Interna, Ospedale Villa Sofia, Palermo, Italia
}

\section{Introduzione}

La depressione, pur essendo tra le entità patologiche più diffuse nella popolazione anziana, è anche una delle meno riconosciute e di conseguenza meno trattate.

La diagnosi può essere confusa con altri disturbi associati all'invecchiamento, oppure, può essere erroneamente accettata come una normale parte del processo di invecchiamento.

La presentazione e i sintomi della depressione possono essere diversi rispetto ai giovani adulti in quanto gli anziani hanno meno probabilità di mostrare sintomi affettivi (ad es. disforia, inutilità e senso di colpa), ed è più probabile che mostrino cambiamenti cognitivi, sintomi somatici (disturbi del sonno, agitazione e perdita generale di interesse). Questi sintomi e le loro modalità di presentazione possono essere attribuiti ad altri disturbi e questo spesso rappresenta un problema in termini di diagnosi differenziale.

Spesso la persona anziana è riluttante a rivolgersi al medico per sintomi di natura psicologica. In molte persone anziane si può rilevare una condizione denominata depressione mascherata caratterizzata da differenti sintomi somatici quali perdita dell'appetito, perdita di peso, riduzione della libido, stipsi e disturbi del sonno che non hanno una spiegazione su base organica. Questi soggetti non riportano esplicitamente tono dell'umore depresso, in quanto hanno difficoltà a verbalizzarlo o si vergognano di soffrire di un disturbo psicologico e/o di avere problemi relazionali e comportamentali e utilizzano il sintomo fisico come strumento di avvicinamento relazionale al medico. ${ }^{1}$

Un altro ostacolo alla corretta identificazione del disturbo depressivo è dovuto al fatto che nella persona

Corrispondente: Salvatore La Carrubba, Medicina Interna, Ospedale Villa Sofia, Palermo, Italia. E-mail: salvatore.lacarrubba@gmail.com

Articolo pubblicato secondo la Creative Commons Attribution NonCommercial 4.0 License (CC BY-NC 4.0).

CCopyright E. Pecoraro e S. La Carrubba, 2019

Licensee PAGEPress, Italy

QUADERNI - Italian Journal of Medicine 2019; 7(4):48-53 anziana la sintomatologia depressiva si sovrappone a differenti patologie coesistenti. ${ }^{2,3} \mathrm{Nel}$ soggetto anziano si possono individuare diverse condizioni d'interesse internistico o neurologico che includono nel loro quadro fenomenologico disturbi depressivi. In particolare la depressione senile si associa a malattie del sistema nervoso centrale (M. di Parkinson, ictus, epilessia, M. di Huntington, traumi cranici e emorragia subaracnoidea), a disturbi endocrini (ipotiroidismo, diabete mellito, $\mathrm{M}$. di Addison, M. di Cushing e iperparatiroidismo), a neoplasie cerebrali, polmonari, renali e altre condizioni cliniche (IMA, LES, fibromialgia, artrite reumatoide e infezioni virali). ${ }^{2}$ Molteplici sono le cause che determinano questo fenomeno, alcune riconducibili a motivazioni di tipo strettamente nosologico altre, invece, legate a condizioni ambientali e sociali che determinano il ritardo di diagnosi e cura per difficoltà, da parte dei familiari e dei medici curanti, di cogliere in tempo $i$ segni iniziali della malattia.

La depressione ha un impatto diverso sulle persone anziane rispetto ai giovani, in particolare, la depressione prolunga $\mathrm{i}$ tempi e le capacità di riabilitazione clinica inficiando negativamente sull'outcome clinico. ${ }^{4}$

Studi condotti su pazienti ricoverati in case di cura per patologie organiche hanno dimostrato che la presenza di depressione aumenta sostanzialmente la probabilità di morte per queste malattie, e per questo motivo, è importante assicurarsi che una persona anziana venga valutata e trattata anche se la depressione è lieve. ${ }^{5}$ Diversi sono gli studi che dimostrano che la depressione aumenta anche il rischio di suicidio, soprattutto negli uomini anziani. Il tasso di suicidi nelle persone di età compresa tra 80 e 84 è infatti più del doppio di quello della popolazione generale. ${ }^{6}$

\section{Epidemiologia}

È stata stimata una prevalenza nei soggetti di età superiore a 65 anni tra 1'1\% e il $35 \% .^{7}$ Questa condizione si presenta in particolar modo tra le persone che risiedono in case di riposo o in istituti di lungodegenza. ${ }^{8}$ L'aumento della popolazione anziana ha determinato un aumento dei disturbi depressivi associati ad altre patologie organiche riconosciute che spesso sono prevalenti; pertanto ciò ha comportato una ulte- 
riore difficoltà nel loro riconoscimento. Secondo l'ultimo rapporto ISTAT (2018), sono oltre 2,8 milioni (il $5,4 \%$ della popolazione con oltre 15 anni) gli italiani che soffrono di depressione e la malattia è in aumento tra gli anziani. Nonostante l'Italia sia uno dei paesi dell'Unione Europea con il minor numero di individui depressi (5,5\% contro il 7,1\% la media Ue), tra gli over 65 il valore raddoppia (l'11,6\% contro $\left.1^{\prime} 8,8 \%\right){ }^{9}$ $\mathrm{Al}$ momento attuale numerosi ricercatori stanno conducendo studi epidemiologici per meglio determinare il grado di rischio che la depressione aggiunge ad altri fattori quali il fumo e l'obesità. Varie sono le ipotesi su tali associazioni, se siano ad esempio legate a scarsa compliance alla terapia o se sussistano invece meccanismi biologici. Inoltre, è ormai stato ampiamente dimostrato che la depressione aumenta il rischio di mortalità per causa cardiaca in soggetti con e senza cardiopatia di base. ${ }^{5}$ D'altra parte va anche considerato che diversi studi condotti su larga scala hanno evidenziato una prevalenza del $15-25 \%$ di disturbi di tipo mentale nei pazienti con una età superiore ai 65 anni. ${ }^{10-14}$ Uno studio condotto dalla FADOI in 1947 pazienti ricoverati nelle Unità Operative di Medicina Interna in Sicilia, ha mostrato che disturbi depressivi non precedentemente diagnosticati erano presenti in 509 pazienti $(26,1 \%)$ e che tali disturbi erano associati a comorbilità internistiche quali ipertensione arteriosa (odds ratio [OR] 1,45; intervallo di confidenza [IC] 95\% 1,18-1,79), diabete mellito (OR 1,48 , IC $95 \% 1,17-1,87$ ), vasculopatia cerebrale (OR 1,50, IC 95\% 1,08-2,07), cirrosi epatica (OR 1,49, IC $95 \% 1,01-2,19)$, e condizioni di disabilità; inoltre, in tale studio fattori predittivi indipendenti per tali disturbi depressivi erano l'età (OR 1,02, IC 95\% 1,011,02 ), il sesso femminile (OR 2,29, IC 95\% $1,83-2,87)$, e la disabilità alle attività strumentali (OR $0,86$, IC $95 \% 0,81-0,93) .{ }^{15}$

\section{Cause della depressione nel paziente anziano}

Il fatto che almeno la metà degli adulti più anziani che si presentano con la depressione non abbia precedenti, suggerisce che possano essere coinvolti diversi meccanismi patologici rispetto ai soggetti che hanno sperimentato in età giovanile disturbi depressivi. ${ }^{16}$ D'altra parte, le persone anziane con depressione che hanno avuto un episodio in età precoce hanno maggiori probabilità di avere una storia familiare di malattia mentale. Diverse condizioni patologiche sono associate ai disturbi depressivi; una depressione può comparire dopo un ictus (20-25\% dei pazienti) e tutti i pazienti in fase post-acuta dovrebbero essere sottoposti a test di screening. Anche un ictus minore o un attacco ischemico transitorio (TIA) può scatenare un grave attacco di depressione. La malattia di Parkinson spesso coesiste con la depressione, e i sintomi depressivi possono a volte preludere all'esordio conclamato della malattia; correlazione molto significativa esiste con la cardiopatia ischemica arrivando ad una prevalenza del 20-25\%; Nei pazienti con diabete mellito di tipo 2 tale correlazione può essere riconosciuta fino al $20 \%$; tra le condizioni neuropsichiatriche, insonnia e disturbi del sonno sono riconosciuti sintomi di depressione, ma tra gli anziani l'insonnia sembra essere anche un fattore di rischio sia per l'insorgenza che per la persistenza dei sintomi depressivi.

\section{Modalità di presentazione della depressione nel paziente anziano e diagnosi}

La depressione in tarda età mostra manifestazioni cliniche ${ }^{16}$ diverse rispetto a quella che insorge in età più precoce, in quanto ha una maggior tendenza alla cronicizzazione, risponde con più difficoltà alla terapia e spesso risulta molto difficile da contrastare. L'irrequietezza motoria si associa frequentemente a sentimenti d'ansia molto accentuata e spesso somatizzata, a timori ipocondriaci con l'ossessione della paura della morte, a contenuti depressivi relativi alla disabilità e alla perdita di autonomia e a idee deliranti centrate sulla convinzione di essere vittima di furti, tradimenti o maltrattamenti. L'associazione con stati d'ansia connota una patologia depressiva di maggior grado di severità e induce una sua più lenta risposta ai trattamenti farmacologici. ${ }^{17}$ Sono frequenti i disturbi della percezione quali illusioni e allucinazioni. In molti casi il soggetto con depressione senile lamenta alterazioni cognitive e circa il 20-50\% degli individui affetti presenta una compromissione cognitiva superiore rispetto ad altri soggetti di pari età e scolarità. ${ }^{18}$

Le prestazioni dei pazienti anziani depressi nei test neuropsicologici che valutano differenti domini cognitivi sono peggiori rispetto a quelle di soggetti sani di pari età e scolarità. Tra le capacità maggiormente compromesse sono la velocità di contestualizzazione delle informazioni, le abilità visuo-spaziali e le abilità esecutive, ${ }^{19}$ inoltre si associa anche un incremento del rischio suicidario soprattutto sopra i 70 anni ed in presenza di disabilità funzionali. ${ }^{20}$ In questo ambito i fattori socio-ambientali risultano essere rilevanti in quanto la maggior parte dei pazienti che commettono atti di suicidio vivono in condizioni di isolamento e di solitudine. ${ }^{21}$ In questi soggetti l'ideazione suicidaria non viene riferita allo specialista e, raramente, viene richiesto un aiuto o un supporto psicologico. ${ }^{22}$

La Risonanza Magnetica ha permesso di evidenziare che la malattia cerebrovascolare si associa alla depressione, tanto da definire una forma di depressione vascolare, spesso associata ad elevato rischio cardiovascolare ed a ipertensione arteriosa, ponendo anche il suggerimento di un maggior rischio di evoluzione verso forme di demenza. ${ }^{23,24}$

Nei soggetti anziani la depressione è spesso associata all'uso di determinati farmaci motivo per cui è 
fondamentale raccogliere una adeguata anamnesi non limitandosi ai farmaci soggetti a prescrizione medica, ma anche farmaci da banco e all'uso di farmaci da medicina alternativa. ${ }^{25}$ Tra i farmaci che possono causare o aggravare la depressione ci sono benzodiazepine, oppioidi, antipsicotici, beta-bloccanti, corticosteroidi (possono causare una serie di reazioni psichiatriche da psicosi), anticonvulsivi, inclusi gabapentin e carbamazepina, FANS, agenti antiparkinsoniani, in particolare levodopa, ranitidina.

L'approccio clinico alla depressione impone un'attenta valutazione dei fattori di rischio, delle comorbilità e dei fattori eziologici associati, valutandone la gravità, il rischio di autolesionismo e il livello di disfunzione. Inoltre, è fondamentale stabilire una buona alleanza terapeutica con il paziente. Molti pazienti anziani con depressione spesso tendono a riportare più sintomi somatici e cognitivi rispetto ai sintomi affettivi. ${ }^{26}$ Vari approcci sono stati usati per diagnosticare la depressione in pazienti con comorbilità. Un approccio esclusivo non considera i sintomi neurovegetativi (ad esempio, cambiamenti nel sonno, energia, appetito e peso), mentre un approccio inclusivo presume che tutti i sintomi contribuiscano all'episodio depressivo, indipendentemente dalla causa. In generale, si suggerisce che un approccio inclusivo possa essere preferibile nei pazienti anziani con patologie internistiche. ${ }^{27} \mathrm{Un}$ aspetto importante per la valutazione della depressione negli anziani riguarda anche la valutazione delle carenze nutrizionali che possono essere responsabili dei sintomi depressivi e la correzione di questi può essere sufficiente per gestire i sintomi depressivi. ${ }^{28}$ Deve essere prestata una dovuta attenzione anche ai fattori psicosociali che possono essere associati all'insorgenza, al peggioramento ed al mantenimento della depressione, tra i quali la solitudine, lo scarso sostegno sociale e familiare, l'assenza di interazione sociale, lo stato di dipendenza, eventuali eventi stressanti, la percezione di cattiva salute, la mancanza di hobby, abitudini alimentari irregolari, l'uso di sostanze e fumo, un livello basso di spiritualità. ${ }^{27}$ Spesso si attribuiscono sindromi psichiatriche a quadri depressivi, ma prima di considerare una diagnosi di tale genere è importante accertarsi che il paziente non abbia un disturbo bipolare. ${ }^{29}$

Per la valutazione dei sintomi depressivi nella popolazione anziana sono state create scale apposite che considerano anche l'eventuale compromissione cognitiva. I questionari disponibili includono Geriatric Depression Scale (GDS), Evans Liverpool Depression Rating Scale (ELDRS), Brief Assessment Schedule (BASDEC) e Patient Health Questionnaire (PHQ-9), la scala di valutazione Hamilton per la depressione (HAM-D). Tuttavia, è importante notare che si tratta di questionari/scale di screening e che sarà necessario un colloquio dettagliato per confermare la diagnosi. ${ }^{30-58}$

\section{Terapia}

Il trattamento della patologia depressiva nel paziente anziano, di base, non è differente da quello dei giovani adulti, e pertanto, i principali strumenti terapeutici sono la terapia farmacologica e la psicoterapia.

Prima di impostare una terapia farmacologica antidepressiva è fondamentale conoscere eventuali farmaci che il paziente già assume, tenendo conto degli eventuali effetti d'interazione tra i farmaci prescritti e quelli già assunti. Per quanto riguarda la classe dei farmaci antidepressivi, numerosi studi confermano che $\mathrm{i}$ triciclici, molto efficaci, sono poco usati nel trattamento del soggetto anziano in quanto, in relazione alla ridotta azione colinergica, il paziente è maggiormente vulnerabile agli effetti anticolinergici centrali e periferici (disturbi cognitivi, ritenzione urinaria, stipsi, disturbi della visione e tachicardia). ${ }^{59}$ Un altro problema connesso all'utilizzo degli antidepressivi triciclici è il pericolo di eventi fatali in caso di assunzione di grosse quantità con finalità suicidarie, condizione, come già descritto, ad elevato rischio nell'anziano depresso. ${ }^{60}$

Una classe di farmaci che si è rivelata essere meglio tollerata per la popolazione anziana è costituita dai serotoninergici selettivi (SSRI), con un rischio di effetti collaterali (nausea, gastralgia, insonnia e irritabilità) di minore allarme clinico. Per quanto riguarda le modalità di somministrazione è molto importante iniziare da un dosaggio molto basso per poi incrementarlo lentamente. Inoltre è fondamentale accertare la corretta comprensione delle indicazioni terapeutiche da parte del paziente, soprattutto nei casi in cui non sia presente un familiare che ne gestisca l'assunzione, tenendo conto che il periodo di assunzione può arrivare a $9-12$ mesi. ${ }^{59}$

Dal punto di vista psicoterapico una delle tecniche maggiormente indicate risulta essere la Terapia del problem solving (PST) ${ }^{61}$ Questo approccio sembra essere efficace, nel trattamento di soggetti anziani affetti da depressione con lieve compromissione cognitiva, nel ridurre i sintomi depressivi e la disabilità. ${ }^{62-64} \mathrm{Un}$ altro approccio che si è riscontrato essere efficace nel trattamento della depressione senile è la terapia cognitivo-comportamentale. ${ }^{65}$

La formulazione del piano di trattamento comporta una decisione, in primo luogo, della strategia terapeutica, quindi sulla scelta degli eventuali farmaci da prescrivere e sugli interventi psicologici da utilizzare. Gli stessi pazienti dovrebbero essere coinvolti nella preparazione del piano di trattamento, ovvero, in determinati casi, coinvolgendo i caregivers, $\mathrm{i}$ quali assumono un ruolo ancora più importante quando il paziente non è in condizione di partecipare alle decisioni terapeutiche sia a causa della gravità della depressione che per una marcata compromissione cognitiva. 


\section{Gestione della depressione da parte dell'internista}

Il paziente anziano, in relazione alle comorbilità, all'aumentata aspettativa di vita, ed alla polifarmacologia è un paziente molto spesso seguito da un internista, che però non può non tener conto della presenza dei disturbi depressivi, delle interferenze tra comorbiltà e stato dell'umore. Nel management clinico, più che in altre condizioni patologiche, è importante stabilire un'alleanza terapeutica. La depressione, infatti, spesso ha un andamento cronico e richiede ai pazienti di partecipare attivamente e aderire al trattamento per lunghi periodi. Un altro aspetto importante per un trattamento di successo è cercare di tollerare eventuali effetti collaterali. Per questi motivi, una forte alleanza medico/paziente è cruciale ed i medici, dovrebbero prestare sempre più attenzione alle preoccupazioni dei pazienti e delle loro famiglie. La gestione della depressione richiede una valutazione continua del decorso dei sintomi e della compliance al trattamento. Di conseguenza, è importante tenere sotto controllo impulsi distruttivi verso sé stessi o altri ed in alcuni casi può essere necessaria una ospedalizzazione $\mathrm{o}$ un trattamento più intensivo. Tutti i pazienti e i loro caregivers devono essere educati sui sintomi della depressione, sulle modalità di trattamento disponibili, sul decorso del disturbo e sul tempo di risposta al trattamento. Elementi educativi specifici possono essere utili in alcune circostanze, ad es. sottolineando che la depressione è una malattia reale e che sono disponibili trattamenti efficaci. Una corretta informazione riguardante le opzioni di trattamento disponibili aiuterà i pazienti a prendere decisioni adeguate, anticipare gli effetti collaterali e favorire l'adesione ai trattamenti.

Molti pazienti anziani possono rifiutare la diagnosi, o temono di diventare dipendenti da psicofarmaci. A volte, i pazienti che sono apertamente sintomatici possono essere scarsamente motivati ed eccessivamente pessimisti circa le loro possibilità di miglioramento con il trattamento. D'altro canto, molti pazienti che ottengono una remissione clinica dopo terapia possono sottostimare la necessità di continuare il trattamento $\mathrm{e}$ considerarlo un peso. Alcuni pazienti potrebbero non essere in grado di prendersi cura di se stessi a causa di deficit cognitivi. I pazienti e i familiari devono anche essere informati sulle possibilità di recidiva dopo un miglioramento.

\section{Qualità di vita}

La depressione senile ha un forte impatto sulla funzionalità quotidiana, sulla qualità di vita e sull'aumento della richiesta di assistenza qualificata. D'altra parte, data l'eterogeneità dei sintomi, in molti casi lo stato depressivo non viene correttamente individuato e di conseguenza trattato. In molte persone anziane si può rilevare una condizione denominata depressione mascherata caratterizzata da differenti sintomi somatici quali perdita dell'appetito, perdita di peso, riduzione della libido, stipsi e disturbi del sonno che non hanno una spiegazione su base organica. Questi soggetti non riportano esplicitamente tono dell'umore depresso, in quanto hanno difficoltà a verbalizzarlo o si vergognano di soffrire di un disturbo psicologico e/o di avere problemi relazionali e comportamentali e utilizzano il sintomo fisico come strumento di avvicinamento relazionale al medico. ${ }^{66}$

\section{Aspetti economico-sociali}

Stabilire i costi a carico della comunità è impresa assai ardua in relazione ai molteplici fattori che intervengono, pur tuttavia i dati disponibili mostrano l'enorme impatto che tale condizione comporta nei paesi occidentali. ${ }^{67,68}$ I costi della depressione, infatti, non sono solo quelli più immediati da intuire, vale a dire ricoveri e terapie, vi sono anche costi sommersi, più nascosti: assenza dal lavoro, sussidi, perdita di produttività. Il vero peso economico della depressione è rappresentato dai costi indiretti, di cui le imprese pubbliche e private sopportano una quota tra il 30 e il 50 per cento: il disturbo incide pesantemente sulla produttività di chi ne soffre, aumentando $i$ costi per le politiche sociali e di welfare. In Europa un lavoratore su dieci si assenta dal lavoro a causa della depressione, per un totale di 21 mila giorni di lavoro persi.

D'altra parte la depressione porta a mancanza di concentrazione, indecisione, perdite di memoria che possono rendere la giornata di lavoro un vero incubo. È quello che gli esperti chiamano presenteeism, ovvero la presenza sul luogo di lavoro in condizioni di salute non ottimali: un fenomeno che secondo alcune stime potrebbe avere costi anche cinque volte superiori a quelli dell'assenteismo vero e proprio. Secondo la ricerca IDEA (Impact of Depression in the Workplace in Europe Audit), che ha coinvolto in tutta Europa oltre 7 mila adulti fra i 16 e i 64 anni, lavoratori e dirigenti, ben il $20 \%$ degli intervistati aveva avuto una diagnosi di depressione e il numero medio di giornate di congedo dal lavoro durante l'ultimo episodio di depressione è stato di 36 giorni. ${ }^{69}$ In Italia, l'assistenza territoriale psichiatrica assorbe il 3,2\% della spesa sanitaria, ed i costi per l'assistenza sanitaria territoriale psichiatrica ammontano nel 2016 a 3,6 miliardi di euro, con un'incidenza sulla spesa sanitaria pubblica totale pari al 3,2\%, secondo stime pubblicate dal Ministero della salute. ${ }^{9}$ I dati rilevati, pur tuttavia, non permettono di distinguere i costi per la depressione estrapolati da tutte le malattie psichiatriche, ma si può intuire che tale peso sia significativo anche se non quantizzabile. Ulteriori osservazioni potrebbero 
fornire indicazioni allo scopo di intervenire in maniera più appropriata allo scopo di abbattere non solo i costi diretti ma i costi indiretti che tale condizione comporta. Tenendo conto del costante aumento dei costi, clinici ed economici a carico dei servizi sanitari, si impone la necessità di una attenzione sempre più crescente e risulta di elevato valore strategico mettere in atto meccanismi di prevenzione e di cura più efficaci coinvolgendo in maniera più attenta le istituzioni politico-sanitarie. ${ }^{70}$

\section{Bibliografia}

1. Alexopoulos GS. Comorbidity of late-life depression: an opportunity for research in mechanisms and treatment. Biol Psychiatry 2002;52:543-58.

2. Lenze EJ, Mulsant BH, Shear MK, et al. Comorbid anxiety disorders in depressed elderly patients. Am J Psychiatry $2000 ; 157: 722-8$.

3. Lenze EJ, Mulsant BH, Shear MK, et al. Comorbidity of depression and anxiety disorders in later life. Depress Anxiety 2001;14:86-93.

4. Lebowitz BD, Pearson JL, Schneider LS, et al. Diagnosis and treatment of depression in late life. Consensus statement update. JAMA 1997:1186-90.

5. Sheline YI, Barch DM, Garcia K, et al. Cognitive function in late life depression: Relationships to depression severity, cerebrovascular risk factorsand processing speed. Biol Psychiatr 2006;60:58-65.

6. Hawton K, Casañas I, Comabella C, et al. Risk factors for suicide in individuals with depression: a systematic review. J Affect Disord 2013;147:17-28.

7. Djernes JK. Prevalence and predictors of depression in populations of elderly: a review. Acta Psychiatr Scand 2006;113:372-87.

8. Covinsky KE, Fortinsky RH, Palmer RM, et al. Relation between symptoms of depression and health status outcomes in acutely ill hospitalized older persons. Ann Intern Med 1997;126:417-25.

9. ISTAT. LA salute mentale nelle varie fasi della vita; 26 luglio 2018. Disponibile a: https://www.istat.it/it/archivio/ 219807

10. Kaufmann MW. Depression, hostility, gender, and myocardial infarction. Program and abstracts from the 153rd Annual American Psychiatric Association Meeting, May 13-18, 2000; Chicago, Illinois. Abstract 28.

11. Simon GE, Von Korff M, Ustun TB, et al. Is the lifetime risk of depression actually increasing? J Clin Epidemiol 1995;48:1109-18.

12. Small GW. Recognition and treatment of depression in the elderly. J Clin Psychiatry 1991;52::S11-22.

13. Chong MY, Tsang HY, Chen CS, et al. Community study of depression in old age in Taiwan: prevalence, life events and socio-demographic correlates. Br J Psychiatry 2001;178:29-35.

14. Reifler BN. Depression: diagnosis and comorbidity. In: Schneider LS, Reynolds CF, Lebowitz BD, Friedhoff AJ (eds). Diagnosis and Treatment of Depression in Late Life. Washington DC: American Psychiatric Press; 1994. pp 55-59.

15. La Carrubba, Manna L, Rinollo C, et al. Depressive symptoms and disability in acute patients with comor- bidities in departments of internal medicine. Ital $\mathrm{J}$ Med 2011;5:261-8.

16. Cole MG, Dendukuri N. Risk factors for depression among elderly community subjects: a systematic review and metaanalysis. Am J Psychiatry 2003;160:1147-56.

17. Beekman AT, de Beurs E, van Balkom AJ, et al. Anxiety and depression in later life: Co-occurrence and communality of risk factors. Am J Psychiatry 2000;157:89-95.

18. Butters MA, Whyte EM, Nebes RD, et al. The nature and determinants of neuropsychological functioning in latelife depression. Arch Gen Psychiatry 2004;61:587-95.

19. Lezak MD. Neuropsychological assessment. New York: Oxford University Press; 1976.

20. Conwell Y, Lyness JM, Duberstein P, et al. Completed suicide among older patients in primary care practices: a controlled study. J Am Geriatr Soc 2000;48:23-9.

21. Conner KR, Duberstein PR, Conwell Y. The validity of proxy-based data in suicide research: a study of patients 50 years of age and older who attempted suicide. I. Psychiatric diagnoses. Acta Psychiatr Scand 2001;104:204-9.

22. Pearson JL, Brown GK. Suicide prevention in late life: directions for science and practice. Clin Psychol Rev 2000;20:685-705.

23. Olin JT, Schneider LS, Katz IR, et al. Provisional diagnostic criteria for depression of Alzheimer disease. Am J Geriatr Psychiatry 2002;10:125-8.

24. Laidlaw K, Davidson K, Toner H, et al. A randomised controlled trial of cognitive behaviour therapy vs treatment as usual in the treatment of mild to moderate late life depression. Int J Geriatr Psychiatry 2008;23:843-50.

25. Sheline YI, Barch DM, Garcia K, et al. Cognitive function in late life depression: Relationships to depression severity, cerebrovascular risk factors and processing speed. Biol Psychiat 2006;60:58-65.

26. Barry KL, Fleming MF, Manwell LB, et al. Prevalence of and factors associated with current and lifetime depression in older adult primary care patients. Fam Med 1998;30:366-71.

27. American Psychiatric Association. Diagnostic and statistical manual of mental disorders: 5th edn. Washington, DC: American Psychiatric Association; 2013.

28. German L, Kahana C, Rosenfeld V, et al. Depressive symptoms are associated with food insufficiency and nutritional deficiencies in poor community-dwelling elderly people. J Nutr Health Aging 2011;15:3-8.

29. Moreno C, Hasin DS, Arango C, et al. Depression in bipolar disorder versus major depressive disorder: results from the National Epidemiologic Survey on Alcohol and Related Conditions. Bipolar Disord 2012;14: 271-82.

30. Reifler BN. Depression: diagnosis and comorbidity. In: Schneider LS, Reynolds CF, Lebowitz BD, Friedhoff AJ (eds). Diagnosis and Treatment of Depression in Late Life. Washington DC: American Psychiatric Press; 1994. pp 55-59.

31. Applegate WB. Use of assessment instruments in clinical settings. J Am Geriatr Soc 1987;34:45-50

32. Zung WW. A self-rating depression scale. Arch Gen Psychiatry 1965:12:63-70.

33. Clayton AH, Holroyd S, Sheldon-Keller A. Geriatric Depression Scale vs Hamilton Rating Scale for Depression in a sample of anxiety patients. Clin Gerontologist 1997;17:3-13.

34. Kitchell MA, Bernes RF, Veith RC, et al. Screening for 
depression in hospitalized geriatric medical patients. J Am Geriatr Soc. 1982;30:174-7.

35. Okimoto JT, Barnes RF, Veith RC, et al. Screening for depression in geriatric medical patients. Am J Psychiatry 1982;139:799-802.

36. Norris JT, Gallagher D, Wilson A, et al. Assessment of depression in geriatric medical outpatients: the validity of two screening measures. J Am Geriatr Soc 1987;35:989-95.

37. Maier W, Philipp M. Comparative analysis of observer depression scales. Acta Psychiatr Scand 1985;72:239-45.

38. Zgourides G, Spofford M, Doppett L. The Geriatric Depression Scale: discriminant validity and elderly daytreatment clients. Psychol Rep 1989;64:1082.

39. Lichtenberg PA, Marcopulos BA, Steiner DA, et al. Comparison of the Hamilton Depression Rating Scale and the Geriatric Depression Scale: detection of depression in dementia patients. Psychol Rep (United States) 1992;70:515-21.

40. Kafonek SD, Roca RP. Proper scoring of the Geriatric Depression Scale (letter). J Am Geriatr Soc 1989;37:819-20.

41. Parmalee PA, Lawton MP, Katz IR. Psychometric properties of the Geriatric Depression Scale among the institutionalized aged. Psychol Assess 1989;4:331-8.

42. Lesher EL. Validation of the Geriatric Depression Scale among nursing home residents. Clin Gerontologist 1986;4:21.

43. Hickie C, Snowdon J. Depression scales for the elderly: GDS, Gilleard, Zung. Clin Gerontologist 1987;6:51.

44. Kafonek S, Ettinger WH, Roca R, et al. Instruments for screening for depression and dementia in a long-term care facility. J Am Geriatr Soc 1989;37:29-34.

45. Folsten MF, Folsten SE, McHugh PR. "Mini-mental state". A practical method for grading the cognitive state of patients for the clinician. J Psychiatr Res 1975;12: 189-98.

46. McGivney SA, Mulvihill M, Taylor B. Validating the GDS depression screen in the nursing home. J Am Geriatr Soc 1994;42:490-2.

47. Burke WJ, Houston MJ, Boust SJ, et al. Use of the Geriatric Depression Scale in dementia of the Alzheimer's type. J Am Geriatr Soc 1989;37:856-60.

48. Burke WJ, Nitcher RL, Roccaforte WH, et al. A prospective evaluation of the Geriatric Depression Scale in an outpatient geriatric assessment center. J Am Geriatr Soc 1992;40:1227-30.

49. Izal M, Montorio I. Adaptation of the Geriatric Depression Scale in Spain: a preliminary study. Clin Gerontologist 1993;13:83-91.

50. Burke WJ, Roccaforte WH, Wengel SP, et al. The reliability and validity of the Geriatric Depression Rating Scale administered by telephone. J Am Geriatr Soc 1995;43:674-9.

51. Nitcher RL, Burke WJ, Roccaforte WH, Wengel SP. A collateral source version of the Geriatric Depression Rating Scale. Am J Geriatr Psychiatry 1993;1;143-52.

52. Sheikh JI, Yesavage JA. Geriatric Depression Scale (GDS): recent evidence and development of a shorter version. In: Clinical Gerontology: A Guide to Assessment and Intervention. New York, NY: The Haworth Press; 1986. pp 165-173.

53. Herrman N, Mittmann N, Silver IL, et al. A validation study of the Geriatric Depression Scale short form. Int J Geriatr Psychiatry 1996;11:451-60.

54. Lesher EL, Berryhill JS. Validation of the Geriatric De- pression Scale - Short Form among inpatients. J Clin Psychol 1994;50:256-60.

55. D'Ath P, Katona P, Mullan E, et al. Screening, detection and management of depression in elderly primary care attendees I: The acceptability and performance of the 15 Item Geriatric Depression Scale (GDS15) and the development of short versions. Family Pract 1994;1:260-6.

56. Scogin F. The concurrent validity of the Geriatric Depression Scale with depressed older adults. Clin Gerontologist 1987;7:23-3.

57. Ferraro FR, Chelminski IJ. Preliminary normative data on the Geriatric Depression Scale-Short Form (GDS$\mathrm{SF}$ ) in a young adult sample. J Clin Psychol 1996;52:443-7.

58. Zalsman G, Weizman A, Carel CA, et al. Geriatric Depression Scale (GDS-15): a sensitive and convenient instrument for measuring depression in young anorexic patients. J Nerv Ment Dis (United States) 2001;189:338-9.

59. Sultana J, Spina E, Trifirò G. Antidepressant use in the elderly: the role of pharmacodynamics and pharmacokinetics in drug safety. Expert Opin Drug Metab Toxicol 2015;11:883-92.

60. Hawton K, Casañas I, Comabella C, et al. Risk factors for suicide in individuals with depression: a systematic review. J Affect Disord 2013;147:17-28.

61. Alexopoulos GS, Raue P, Arean P. Problem-solving therapy versus supportive therapy in geriatric major depression with executive dysfunction. Am J Geriatr Psychiatry 2003;11:46-52.

62. Alexopoulos GS, Raue PJ, Kiosses DN, et al. Problemsolving therapy and supportive therapy in older adults with major depression and executive dysfunction: effect on disability. Arch Gen Psychiatry 2011;68:33-41.

63. Kiosses DN, Leon AC, Arean PA. Psychosocial interventions for late-life major depression: evidence-based treatments, predictors of treatment outcomes, and moderators of treatment effects. Psychiatr Clin North Am 2011;34:377-401.

64. Arean PA, Raue P, Mackin RS, et al. Problem-solving therapy and supportive therapy in older adults with major depression and executive dysfunction. Am J Psychiatry 2010;167:1391-8.

65. Laidlaw K, Davidson K, Toner H, et al. A randomised controlled trial of cognitive behaviour therapy vs treatment as usual in the treatment of mild to moderate late life depression. Int J Geriatr Psychiatry 2008;23:843-50.

66. Sivertsen H, Bjørkløf GH, Engedal K, et al. Depression and Quality of Life in Older Persons: A Review. Dement Geriatr Cogn Disord 2015;40:311-39.

67. Bock JO, Brettschneider C, Wegener A, et al. Cost-Effectiveness of the Treatment of Depression in Old Age. Psychiatr Prax 2015;42:240-7.

68. Jonsson U, Bertilsson G, Allard P, et al. Psychological Treatment of Depression in People Aged 65 Years and Over: A Systematic Review of Efficacy, Safety, and Cost-Effectiveness. PLoS One 2016;11:8.

69. IDEA: Impact of Depression at Work in Europe Audit Final report. Ipsos Healthcare; October 2012.

70. Zhang Y, Chen Y, Ma L. Depression and cardiovascular disease in elderly: Current understanding. J Clin Neurosci 2018;47:1-5. 


\title{
La sessualità nell'anziano
}

\author{
Vilma Duretto, ${ }^{1}$ Giuliano Pinna ${ }^{2}$ \\ ${ }^{1}$ Psicologa; Pedagogista; Sessuologa Clinica Albo FISS; Sessoanalista (IIS); ${ }^{2}$ Consigliere onorario FADOI Piemonte, Italia
}

\section{Introduzione}

L'Italia è attualmente tra i Paesi del mondo con la più alta percentuale di anziani, seconda al Giappone e seguita dalla Germania. Nel 2050 un terzo della popolazione italiana, avrà un'età superiore ai 65 anni, con una aspettativa di vita media, riportando le più recenti rilevazioni ISTAT del $2017,{ }^{1}$ rispettivamente di 85 anni per le donne e 81 per gli uomini.

Il concetto di salute dell'OMS, presentato nel $1948^{2}$ e ancora universalmente accettato dagli addetti ai lavori, definisce la salute come uno stato di completo benessere fisico, mentale e sociale e non la semplice assenza dello stato di malattia o di infermità.

Sempre l'OMS nel documento tecnico n. 572 del $1975^{3}$ a completamento del concetto di salute, afferma che: la salute sessuale è l'integrazione degli aspetti somatici, affettivi, intellettivi e sociali dell'essere sessuato, che consentono la valorizzazione della persona, della comunicazione e dell'amore.

In altri termini se escludiamo la salute sessuale, viene meno il concetto di salute; per estensione verrebbe meno anche una parte dell'articolo 32 della Costituzione Italiana: La Repubblica tutela la salute come fondamentale diritto dell'individuo e interesse della collettività... ${ }^{4}$

Nel 2015 venne presentata a Torino, nell'ambito di un convegno tra sessuologi clinici e professionisti sul tema della salute sessuale nella coppia over $60,{ }^{5}$ la classificazione di alcuni autori sulle Età della vita, che inizialmente da tre sono passate a quattro, come ci riportano dipinti storici (Tabella 1, ${ }^{6,7}$ Figura 1) per giungere a cinque.

Corrispondente: Vilma Duretto, Strada del Salino 4bis, 10133 Torino, Italia.

E-mail: vilmaduretto@yahoo.it ; vilma.duretto.032@psypec.it

Articolo pubblicato secondo la Creative Commons Attribution NonCommercial 4.0 License (CC BY-NC 4.0).

(C) Copyright V. Duretto e G. Pinna, 2019

Licensee PAGEPress, Italy

QUADERNI - Italian Journal of Medicine 2019; 7(4):54-58

\section{Attività sessuale nell'anziano}

In due revisioni, una del 2007 pubblicata sul New England Journal Medicine ${ }^{8}$ e una del 2011 pubblicata sul Journal Sexual Medicine, ${ }^{9}$ la presenza di un'attività sessuale tra le persone con età compresa tra i 57 e i 64 anni era del 67,5\%, mentre tra le persone di età compresa tra 65 e 74 anni era del $46 \%$, e tra 75 e 85 anni del $24 \%$.

Questi dati ovviamente non definiscono la frequenza dell'attività sessuale: per i sessuologi clinici è decisamente meno significativo il concetto di quantità rispetto a quello di qualità dei rapporti sessuali.

Una realtà molto più vicina a noi, più attuale, è la ricerca su Gli anziani il sesso e l'amore condotta dall'Istituto Internazionale di Sessuologia di Firenze Istituto Ricerca e Formazione S.r.L (www.irf-sessuologia.it), dalla prof.ssa Roberta Giommi, ${ }^{10}$ dove, su una popolazione maschile e femminile compresa tra $\mathrm{i}$ 60 e 75 anni, emerge come alla domanda Lei e il/la suo/a partner fate l'amore?, il 75\% delle femmine e il $68 \%$ dei maschi risponde spesso/qualche volta (Figura 2).

A seguire, alla domanda Ritiene che sia normale l'autoerotismo in età anziana?, tra i 60 e i 65 anni, rispondono Qualche volta il $44 \%$ dei maschi e il $43 \%$ delle femmine (Figura 3).

Nota: l'analisi di attendibilità dei grafici in Figura 2 e 3 è stata compiuta tramite l'utilizzo dell'alpha di Cronbach che verifica la coerenza interna degli item della scala. Il risultato suggerisce una buona coerenza interna, gli item correlano tra loro e contribuiscono tutti insieme a rappresentare il campione di comportamenti osservati.

Eppure vi sono ancora molte false credenze sulla sessualità nella senescenza: sempre nel grafico in Figura 3 si vede come circa il $40 \%$ dei maschi non consideri normale l'autoerotismo.

Per molti infatti: i) meglio non parlare di queste cose ad una certa età; ii) le persone anziane non hanno abilità per fare sesso; iii) la sessualità negli anziani è fastidiosa, grottesca, sporca.

Il concetto di vecchiaia è in continua evoluzione: nel congresso della Società Italiana di Gerontologia e Geriatria svoltosi a Roma nel novembre 2018 è emerso un concetto basilare: si diventa anziani dopo i 
75 anni... un 65enne di oggi ha la forma fisica e cognitiva di un 40-45enne di 30 anni fa... un 75 enne quella di un individuo che aveva 55 anni nel 1989.

Sono numeri che fanno riflettere e fanno sorgere tante domande, ma non certo stupire.

Seneca, al termine della sua vita, a 69 anni, nelle lettere a Lucillo scriveva: L'età avanzata, non ancora decrepita, è molto gradevole e io credo, anche, che chi è arrivato all'estremo limite, abbia i suoi piaceri, perlomeno, gli tien luogo di piacere il fatto di non averne più bisogno. ${ }^{11}$ Nell'opera La Repubblica Platone chiedeva a Socrate: Come ti vanno le cose d'amore? Sei ancora capace di rapporti intimi con una donna?, quegli rispose: Zitto amico, sono proprio contento di essermene liberato, come da un padrone rabbioso e intrattabile. ${ }^{12}$ Platone morì a 80 anni e l'opera sopra citata fu scritta approssimativamente tra i 40 e i 60 anni. I vecchi 60enni Seneca e Socrate sono i diversamente giovani di oggi.

Ora non si invecchia più, piuttosto si cresce, non si aggiungono anni alla vita, si aggiunge vita-lità agli anni; come dice J. Medina, il grande biologo molecolare, nel suo libro Il cervello non ha età. ${ }^{13}$

Questa significativa differenza generazionale è

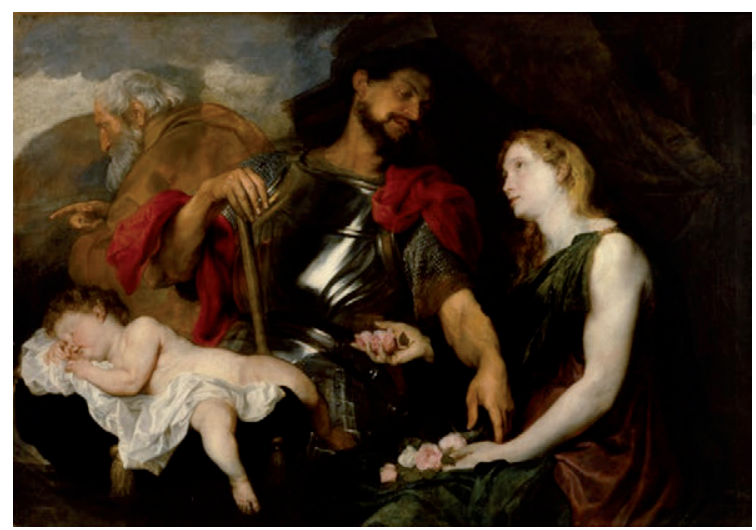

Figura 1. Le quattro età dell'uomo di Antoon van Dyck (Anversa 1599 - Londra 1641) - conservato presso il Museo Civico di Palazzo Chiericati, Vicenza. molto più evidente nel sesso femminile. A sessant'anni oggi una donna è simile ad una quarantenne di una volta, forse a settantacinque anni inizia a sentirsi come si sarebbe percepita sua nonna o bisnonna con venti anni di anticipo.

Sempre più spesso vediamo anche donne mature in affettuosa compagnia di giovani maschi e non solo più uomini brizzolati in compagnia di seducenti giovani donne.

Ma non solo si diventa anziani quindici o venti

\section{Lei ed il suo partner fate l'amore? (60-75)}

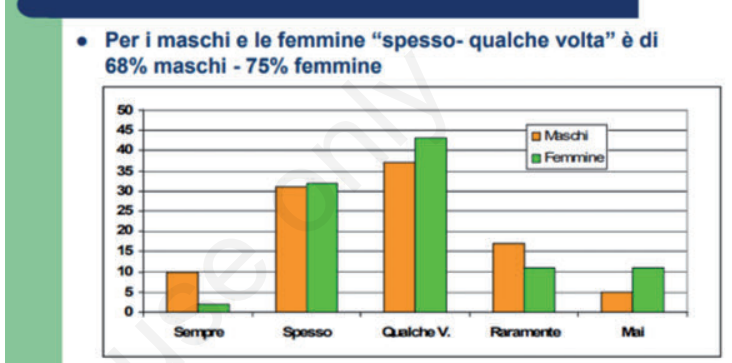

Figura 2. Risposta in base al genere alla domanda: Lei $e$ il/la partner fate l'amore?

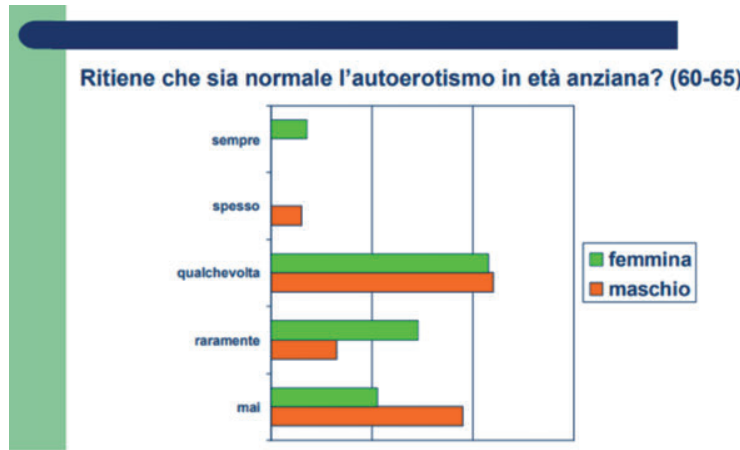

Figura 3. Risposta in base al genere alla domanda: $R i$ tiene che sia normale l'autoerotismo in età anziana?

Tabella 1. Le età della vita.

\begin{tabular}{|c|c|}
\hline $1^{\circ}$ età: dal concepimento-nascita ai 30 anni & GIOVANE \\
\hline $2^{\circ}$ età: dai 30 ai 60 anni & ADULTO \\
\hline $\begin{array}{c}3^{\circ} \text { età: } \text { dai } 60 / 65 \text { agli } 80 \text { anni } \\
\text { dai } 60 \text { ai } 75 \text { anni }\end{array}$ & $\begin{array}{l}\text { ANZIANO (P. Laslet) } \\
\text { ANZIANO }(\text { L. Peirone) })^{7}\end{array}$ \\
\hline $\begin{aligned} 4^{\circ} \text { età: } & \text { dai } 75 \text { ai } 90 \\
& \text { dagli } 80-85 \text { ai } 90\end{aligned}$ & $\begin{array}{l}\text { VECCHIO (L. Peirone) })^{7} \\
\text { VECCHIO (P. Laslet) }\end{array}$ \\
\hline $5^{\circ}$ età: dai 90 ai 100 e oltre & GRANDE VECCHIO \\
\hline
\end{tabular}


anni dopo, lo si diventa in modo diverso: la diversa longevità è una vittoria, sulle malattie, sulle difficoltà della vita, sui pericoli e sulle debolezze.

Da una ricerca del Dipartimento di Geriatria dell'Università di Padova del 2016, emerge che le vedove risultano più in forma delle mogli con un marito ancora in vita. Si sono liberate di un uomo quasi sempre più anziano di loro e spesso vivono una nuova storia d'amore meno impegnativa di un matrimonio. Riscoprono l'amicizia di un'altra donna, si rendono conto come Socrate di essere più libere di un tempo, ma soprattutto diversamente libere. E la sessualità segue questo più lento invecchiare. Anzi, la sessualità non invecchia, se ci intendiamo sul significato di sessualità. Cosa è dunque la sessualità? Un coito? Un bacio? Un abbraccio? Una carezza? Uno sguardo languido? Un soave profumo maschile o femminile? Una parola dolce o appassionata? È tutto questo, perché la sessualità non ha corpo senza la sensualità.

Ancora, cos'è la sessualità? Potremmo considerarla come espressione della comunicazione, come un dono da portare all'altro. Noi nasciamo esseri sessuati, i nostri sensi, alcuni prima, come l'olfatto, altri più tardi, come la vista, ci accompagnano dai primi giorni della nostra vita sino agli ultimi. Così è per la sessualità: quanto nutrono il corpo una carezza affettuosa, un tenero abbraccio, un inebriante profumo,un bacio sulla bocca! Essi trasmettono ad alcune parti del cervello intense sensazioni, vere esplosioni di piacere, di gioia, di serenità: sono un antidepressivo naturale. Ecco che non dobbiamo stupirci se, per esempio, lavare delicatamente il corpo del proprio partner o profumarlo di un gradevole olio sprigiona sensazioni di piacere inebriante: azioni sul corpo piacevoli, piacevolmente modificano i nostri circuiti cerebrali; una parola sentita ed espressa con autentico affetto ha un effetto psicoterapeutico, promuove piacere, benessere, serenità.

È quella che Helen Singer Kaplan, ideatrice della Terapia Mansionale Integrata ${ }^{14}$ definì Focalizzazione Sensoriale. Qual è l'obbiettivo di tutti questi comportamenti? Promuovere il riconoscimento dell'altro, riconoscere attraverso di noi l'altro e ottenerne, in cambio, un riconoscimento, una conferma dell'identità, un vero e proprio rinforzo all'autostima: è un ansiolitico naturalmente prodotto.

L'amore per l'altro passa prima dall'amore per se stessi: prima devo conoscere e riconoscere me stesso (Socrate: conosci te stesso), poi ci saranno il riconoscimento e l'amore per l'altro. Questo percorso amoroso non ha un'età, bensì una diversità nelle età: in qualsiasi epoca della vita, in contesti e culture differenti. Perché le emozioni di base quali paura, rabbia, tristezza, gioia, disgusto, sono universali. Come la musica è linguaggio universale, compreso in tutto il mondo, così è la sessualità: possiamo ascoltare musica jazz, classica, rock, ma tutte si basano sul medesimo pentagramma, su sequenze infinitamente diverse di note.

Devono esistere però delle regole. Come la melodia, l'assonanza, il piacere dell'ascolto richiedono il rispetto di regole precise e imprescindibili, così è per la sessualità: possiamo guardarci, toccarci, ascoltarci, baciarci, ma tutto nel rispetto di regole e comportamenti che permettano un buon funzionamento individuale e relazionale con se stessi e con gli altri. Trasmettere queste regole e correggere i comportamenti è il lavoro della pedagogia e psicologia sessuologica.

Sembra ovvio, ma è necessario ribadire che la prima regola comportamentale da osservare è il valore della riservatezza e dell'intimità, a qualsiasi età.

La sessualità non è un fatto pubblico, non va esposta sul davanzale della finestra come un rigoglioso vaso di gerani, di cui andar fieri, bensì deve essere conservata, custodita, ammirata, percepita e sentita per il noi. Del resto, molti delicati e bellissimi fiori appassiscono se troppo esposti al sole e alle intemperie.

Un concetto molto importante espresso da Zygmunt Bauman, ${ }^{15}$ anche evocato da Jacques Lacan ${ }^{16}$ e recentemente riportato da Massimo Recalcati ${ }^{17}$ è che quando si espone e ci si espone un po' troppo alla finestra, forse è perché la stanza è vuota, perché mi serve la conferma dell'altro per sopperire all'assenza della mia conferma... Un po' come la storia del pescatore o del cacciatore che con racconti di prede fantastiche cercano la propria soddisfazione nello stupore provocato negli altri.

Proprio come il parafilico esibizionista il cui unico godimento è nell'acquisizione visiva dello stupore dell'altro.

Dobbiamo arredare le stanze della nostra vita a qualsiasi età con sane e congrue esperienze di sessualità, non metterle in vetrina: non facciamo entrare chiunque in casa nostra, che avrà sicuramente una porta, una chiave ed un campanello per garantirne la riservatezza.

Nessun accanimento, né farmacologico, né comportamentale, dobbiamo sentire cosa ci fa star bene, senza esibirlo. Essere ciò che si è, nel rispetto delle regole di espressione del corpo dell'altro e del proprio.

Perché quindi dovremmo negare agli anziani, ai vecchi, ai diversamente giovani questi diritti? Seguendo queste idee, dovremmo dunque negare anche ai bambini di abbracciarsi, di baciarsi, di sorridersi, di annusarsi?

Vi sono delle regole, dei limiti che occorre insegnare, trasmettere, che possono essere culturalmente diversi, soprattutto in questa società sempre più riccamente multietnica, ma che sempre rispondono alle emozioni universali in qualsiasi età. Siamo individui sessuati dalla nascita alla morte. Anche quando per età, scelta o impedimenti sono assenti rapporti genitali, la sessualità trova per esprimersi alternative peculiari all'età e alla persona.

Lasciamo quindi nel tempo vivere la diversa e ricca espressione della sessualità che è in noi.

Oggi la sessualità e l'amore nella terza, quarta, quinta età non sembrerebbero essere più negati o addirittura stigmatizzati come un tempo; sempre più numerose sono le nuove coppie di seniores, anche se non sempre formalizzate dal matrimonio o consolidate da una coabitazione. 
Sono modi nuovi di vivere situazioni di coppia con cui il senior cerca di far fronte alla solitudine. La solitudine la si teme ad ogni età. Nella terza età e nelle età a venire è il male peggiore, un male che ha ripercussioni negative sia nel corpo che nella psiche: il lavoro non occupa più lo spazio della quotidianità, i figli hanno lasciato il nido e ancora non è tempo di replicare la bio-geniorialità, alcuni amici coetanei hanno lasciato questo mondo.

Ecco che i rapporti di affettuosa amicizia che nascono nell'ordinaria quotidianità o nei sempre più numerosi contesti ricreativi, culturali e on-line, sui forum, chat, mettono in luce l'esistenza di bisogni di comunicazione profonda, di compagnia, di sessualità, di presenza affettiva: il sano rifiuto della solitudine.

Sembrerebbe che il 70\% degli attuali anziani passa intere giornate senza scambiare una sola parola con un'altra persona.

La vecchiaia non è la pace dei sensi, come molti giovani amano sottolineare, bensì la pace tra i sessi, tra i generi: il rapporto tra donna e uomo, forse lungo una linea di una saggezza maturata in base all'esperienza, risulterebbe essere più equilibrato, con una indubbia maturazione sul piano relazionale. Molti pregiudizi andrebbero affrontati molto presto: ad esempio, una corretta educazione sessuale scolastica potrà fare molto per trasmettere ai bambini e ai giovani ragazzi il messaggio rasserenante di un erotismo che accompagni l'uomo e la donna per tutta la vita. Proprio come scriveva J.P. Sartre nel 1943 nel libro L'essere e il nulla: La sessualità appare con la nascita e scompare con la morte. ${ }^{18}$

Scriveva Gabriel García Márquez nella Lettera d'addio, il suo testamento: Convincerei ogni uomo e ogni donna che sono i miei favoriti e vivrei innamorato dell'amore, dimostrerei agli uomini quanto sbagliano nel pensare che si smette di innamorarsi quando si invecchia, senza sapere che si invecchia quando si smette d'innamorarsi ... ai vecchi insegnerei che la morte non arriva con la vecchiaia ma con l'oblio... ${ }^{19}$

Un autore un po' più recente, quale Giampaolo Pansa nel suo libro Vecchi, folli e ribelli ${ }^{20}$ affronta l'espressione dell'affettiva nella senilità con sana, serena perversa e polimorfa realtà.

\section{Il Grey divorce}

Un fenomeno che secondo le rilevazioni ISTAT del $2010,{ }^{21}$ sembrerebbe essere un trend in crescita, è quello del Grey divorce: 1'11,2\% delle separazioni avviene tra gli over 60 maschi (la rilevazione precedente, 7 anni prima, si assestava sul 5,9\%). Nelle donne over 60 le separazioni avvengono in una percentuale del $6,4 \%$, anche qui trend doppio rispetto a 7 anni prima, pari al 3,6\%.

A quali considerazioni ci portano questi dati? i) Indubbiamente esiste un allentamento della censura sociale: stiamo parlando di generazioni che hanno vissuto la rivoluzione culturale del ' 68 . I maschi, anche e soprattutto in conseguenza dell'epocale emancipazione della donna verificatasi in quel periodo, hanno acquisito competenze tradizionalmente femminili. Ciò ha riguardato anche la loro autonomia e la loro cura. Che dire poi dei nuovi modelli culturali proposti dai media, vedi fiction sui Cesaroni, Dallas, Dinasty o i vari talk-show? ii) La recente disponibilità di nuovi farmaci permette di funzionare meglio e con chi si vuole; iii) Spesso i problemi d'incomprensione tra gli over 60 sono causati da erronee interpretazioni, ovvero si scambia per mutamento del carattere una malattia, una demenza senile, una vasculopatia o Alzheimer... e invece di curarsi si divorzia; iv) infine è decisiva la maggiore indipendenza della donna, che porta a una maggiore autonomia decisionale nel separarsi dal marito padrone.

Alla luce di quanto detto non ci resta che augurarci di poter lungamente e serenamente invecchiare, ricordandoci che chi ben semina ben raccoglie. La sessualità non è improvvisazione, ma un lungo creativo e meditato linguaggio, che non s'improvvisa, ma si costruisce: il trascorrere degli anni non può che renderlo più ricco, articolato, e profondo.

Una proposta a tutte le coppie etero, omo, geometriche, ecc. a trovare il gas nel prosieguo della relazione con i 10 comandamenti per proseguire una serena vita sessuale affettiva relazionale: i) riscoprire e mantenere il valore della seduzione; ii) mantenere e riscoprire la diversità dell'autoeroticità insieme; iii) focalizzare ed esprimere quello che stimola ed inibisce il desiderio sessuale; iv) programmare l'intimità e l'attività sessuale: non è naturale e non s'improvvisa; v) esprimere il piacere verso l'espressione della sessualità; vi) comunicare apertamente i propri bisogni; vii) risolvere assieme i problemi sessuali e affettivo relazionali; viii) sfidare le (false) credenze sulle disfunzioni sessuali; ix) utilizzare in modo corretto e complice i farmaci quando possibile soluzione nelle disfunzioni sessuali; $\mathrm{x}$ ) continuare ad avere una buona relazione e comunicazione (Figura 4).

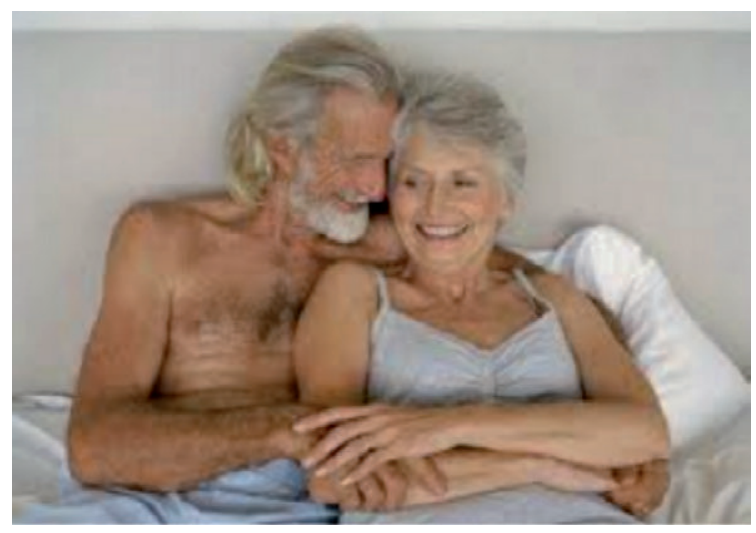

Figura 4. L'importanza di mantenere una serena vita sessuale affettiva relazionale. 
Allacciandosi al decimo comandamento è fondamentale riconoscere che per avere una buona relazione orizzontale è fondamentale continuare a coltivare una buona relazione in verticale; a tal proposito vi invito alla lettura di I dieci comandamenti della coppia a cura di Jeffrey K. Zeig e Tami Kulbatsky, redatti e condivisi da 80 famosi psicologi e psicoterapeuti. ${ }^{22}$

\section{Bibliografia}

1. ISTAT. Indicatori demografici - stime per l'anno 2016; 6 marzo 2017. Disponibile su: www.istat.it

2. FISS, WAS. La salute sessuale per il terzo millennio Dichiarazione e documento tecnico. Disponibile a: www.fissonline.it / www.was.org

3. World Health Organization. $29^{\wedge}$ Assemblea Mondiale della Salute; 29 marzo 1976 - Atti ufficiali. Disponibile su: www.apps.who.int

4. Senato della Repubblica - Costituzione della Repubblica Italiana; dic. 2012. Disponibile su: www.senato.it

5. Duretto V. Aggiungere vita agli anno...quando il meno può valere di più. Atti del Convegno ASST 2015 - La Coppia over 60: le professioni di aiuto si confrontano sul tema della salute sessuale, 24 ottobre 2015, Torino.

6. Laslet P. A fresh map of life. London: Palgrave Macmillan; 1996.

7. Peirone L, Gerardi E. Il sole della sera. Torino: Edizione Antigone; 2009.

8. Tessler Lindau S, Schumm LP, Laumann EO, et al. A study of sexuality and health among older adults in the United States. N Engl Med 2007;357:762-74.

9. Proceedings of the 16th Annual Fall Scientific Meeting of the Sexual Medicine Society of North America. Miami Beach, Florida November 10-14, 2010. J Sexual Med 2011;8:Supplments 1-5.

10. Giommi R. Gli anziani il sesso e l'amore. Istituto Internazionale di Sessuologia di Firenze, Istituto Ricerca e Formazione S.r.L. Disponibile a: www.irf-sessuologia.it

11. Seneca Lucio Anneo. Lettere a Lucillo. I Classici. Forlì: Foschi Editore; 2017.

12. Platone. La Repubblica. Bari: Edizione Laterza; 2007

13. Medina J. Il cervello non ha età. Torino: Ed. Bollati Boringhieri; 2018.

14. Singer Kaplan H. Nuove terapie sessuali. Firenze: Bompiani; 1977.

15. Bauman Z. Amore liquido - sulla fragilità del legami affettivi. Bari: Ed. Laterza; 2006.

16. di Liberto Y. Il pieno e il vuoto: Jacques Lacan, Gilles Deleuze e il tessuto del reale. Nocera: Ed. Orthotes; 2017.

17. Recalcati M. Non è più come prima. Milano: Ed. Raffaello Cortina; 2014.

18. Sartre JP. L'essere e il nulla. Milano: Ed. Il Saggiatore; 2014.

19. García Márquez G. Lettera d'addio (2012 circa, di non pubblicazione e di discussa appartenenza all'autore). Fonte "La Repubblica" - lun. 14 aprile 2014.

20. Pansa G. Vecchi, folli e ribelli - Il piacere della vita nella terza età. Milano: Rizzoli Editore; 2016.

21. ISTAT. Separation and divorces in Italy; Year 2010. Disponibile su: www.istat.it

22. Zeig G, Kulbatsky T. I dieci comandamenti della coppia. Milano: Ed. Saggi Ponte alle Grazie; 2012. 


\title{
Gli ostacoli metodologici nella comunicazione con il paziente anziano
}

\author{
Mario Felici, ${ }^{1}$ Angela Tufi, ${ }^{1}$ Antonio Pulerà, ${ }^{1}$ Salvatore Lenti ${ }^{2}$ \\ ${ }^{1}$ U.O.C. Geriatria; e ${ }^{2}$ U.O.S.D. Ipertensione, dislipidemia e rischio cardiovascolare, Ospedale San Donato di Arezzo, \\ USL Toscana sudest, Arezzo, Italia
}

\section{Introduzione}

Negli ultimi decenni la medicina interna è passata dal curare in prevalenza pazienti con patologia acuta d'organo alla necessità di gestire un numero sempre più crescente di pazienti con riacutizzazioni di patologie croniche multiple e con maggiore fragilità clinica e sociale. Questo cambiamento epidemiologico sanitario ha costretto a ridisegnare i modelli comportamentali e organizzativi dell'assistenza. ${ }^{1}$ L'importanza di acquisire e sviluppare capacità comunicazionali appare ancora più importante per il medico in un contesto nel quale prevalgono le necessità assistenziali di tipo cronico e il successo delle cure è in buona parte affidato anche al contesto socio-familiare nel quale è inserito. ${ }^{2}$ Senza una relazione medico-paziente ben strutturata è difficile pensare che le scelte del paziente possano essere consapevoli e la sua adesione ai percorsi diagnostico-terapeutica sia affidabile e attiva. È sempre più evidente che l'approccio alla cura dovrà risultare rispettoso delle preferenze, dei bisogni e dei valori del singolo paziente, assicurando che questi aspetti guidino le decisioni cliniche. Gli ostacoli inerenti alla comunicazione diretta con il paziente anziano dovuti principalmente a barriere fisiche (deficit sensoriali e cognitivi) e psicologiche (ansia, depressione, bassa autostima) sono molto frequenti nella pratica clinica quotidiana ed ogni medico, con la sua sensibilità e le sue abilità comunicative riesce comunque a superare con efficacia. Gli ostacoli più difficili da superare sono invece quelli dovuti principalmente alla mancata, o errata, pratica metodologica. Ostacoli metodologici inquadrati sia nella più ampia cornice delle communication skills che nella capacità

Corrispondente: Mario Felici, U.O.C. Geriatria, Ospedale San Donato di Arezzo, USL toscana sudest, Arezzo, Italia. E-mail: mariofelici@me.com

Articolo pubblicato secondo la Creative Commons Attribution NonCommercial 4.0 License (CC BY-NC 4.0).

CCopyright M. Felici et al., 2019

Licensee PAGEPress, Italy

QUADERNI - Italian Journal of Medicine 2019; 7(4):59-62 del medico di integrare, nel suo colloquio clinico, la sua agenda (diagnosi e terapia - disease) con l'agenda del paziente (vissuto di malattia - illness) e con l'agenda del familiare (sickness). ${ }^{3}$

\section{Primo ostacolo metodologico: inquadramento del paziente nel giusto contesto clinico}

Il modello di Kurtz e Snowden, applicato alla medicina, permette di definire una medicina della semplicità, una medicina della complessità ed una medicina del caos. ${ }^{4}$ Ogni ambito della medicina è dunque caratterizzato dall'interazione fra le evidenze scientifiche certificate $\mathrm{e}$ la condivisione della comunità medica. Il sapere e la conoscenza fanno quindi riferimento allo specifico contesto. Quindi il primo ostacolo da superare sarà quello di saper inquadrare il paziente, nell'ambito clinico che maggiormente lo rappresenta. Di fatto, l'anziano ricoverato nei reparti di medicina interna è inquadrabile nella cornice teorica della medicina della complessità (buone pratiche cliniche, carenza di solide certezze scientifiche e ambigua condivisione di queste) oppure nella medicina del caos (assenza di evidenze scientifiche e frammentata condivisione di pratiche cliniche per lo più emergenti). In base al contesto clinico si declinerà poi sia il metodo clinico di approccio che il registro comunicativo utilizzato (patient-oriented o person-oriented) (Figura 1).

È facilmente intuibile che se viene a mancare la cornice teorica di riferimento è facile creare barriere alla comunicazione e ancor di più ostacoli nella relazione di fiducia tra medico e paziente e la sua famiglia. La difficolta del clinico nell'affrontare il paziente con condizioni cliniche multiple è stata ben affrontata da Tinetti e collaboratori ${ }^{5}$ in cui si tratteggia con chiarezza il problema dell'interazione fra le indicazioni di due o più linee guida in termini di compatibilità e di scelta da affrontare che logicamente ne deriva. Spesso il medico è costretto a prendere determinate decisioni in condizioni di incertezza per mancanza assoluta di informazioni rilevanti o per cattiva qualità di quelle esistenti, facendo ricorso, più spesso di quanto si pensi, alla sua esperienza clinica. I buoni dottori, sottolinea Cagli ${ }^{6}$ riescono a impiegare l'esperienza clinica e le prove della letteratura 
medica qualificata, ma nessuna di queste componenti può essere sufficiente. Saltare l'inquadramento del contesto clinico equivale a non avere un punto di riferimento dell'ambiente in cui si opera. Viene considerato un ostacolo alla comunicazione/relazione perché sia nella medicina della complessità che del caos, durante il colloquio clinico, devono essere presi in considerazione gli aspetti della malattia percepiti dal punto di vista del paziente, ovvero l'illness. L'esplorazione dell'illness rimane un punto caratterizzante la comunicazione dell'approccio orientato alla persona come portatore di malattia e non identificato con essa. Non dare la giusta importanza a tale dimensione narrativa, soprattutto per l'anziano malato, costituisce senza meno un secondo ostacolo alla comunicazione. Quindi se è vero che la comunicazione non deve essere vista come un processo di trasmissione di informazioni, ciò è ancor più evidente nella relazione con l'anziano, che vuole trasmettere i contenuti soggettivi del suo essere è quindi incline a fornire dettagli e argomentazioni circa la sua storia di malattia. Pertanto, la comunicazione con il paziente, e a maggior ragione con il paziente anziano, deve essere un processo interattivo e l'interazione è completa solo se il medico riceve feedback su come il messaggio è stato interpretato e quali emozioni ha suscitato nel paziente.

\section{Secondo ostacolo metodologico: la valutazione della fragilità}

Per quanto riguarda il contenuto della comunicazione dobbiamo ricordare che per comunicare e con- dividere un piano di vita e di cure con il paziente anziano, dovremo tenere conto delle condizioni del paziente in un contesto di inquadramento fit, frail e vulnerabile. La letteratura più consistente individua la fragilità dell'anziano in una sindrome biologica caratterizzata da riduzione delle riserve e della resistenza agli stress e provocata dal declino cumulativo di più sistemi fisiologici; $;$,8 è quindi ben differenziata dalla disabilità. Si caratterizza inoltre per l'eterogeneità e l'instabilità e per la vulnerabilità con tendenza a manifestazioni peggiorative della salute. Sicuramente vi concorrono fattori molto diversi, di natura biologica, medica, psicologica ed ambientale; anche il suo impatto clinico è condizionato dall'ambiente fisico, sociale e dalle situazioni che perturbano l'equilibrio della vita quotidiana. ${ }^{9} \mathrm{Il}$ concetto di fragilità della persona è stato oggetto di crescente interesse negli ultimi anni, soprattutto in relazione al fenomeno del progressivo invecchiamento della popolazione e benché ampio spazio vi sia stato dedicato dalla letteratura scientifica, non è stato raggiunto ancora un pieno accordo circa i criteri più corretti per identificarlo. Esiste, invece, accordo nel ritenere la fragilità uno stato biologico età-dipendente caratterizzato da ridotta resistenza agli stress, secondario al declino cumulativo di più sistemi fisiologici e correlato a pluripatologia, disabilità, rischio di istituzionalizzazione e mortalità.

Ad oggi la valutazione della performance fisica utilizzando lo Short Physical Performance Battery $(\mathrm{SPPB})^{10} \mathrm{e}$ l'inquadramento secondo il Fenotipo Clinico di L. Fried ${ }^{11}$ permette una semplice ma soddi-

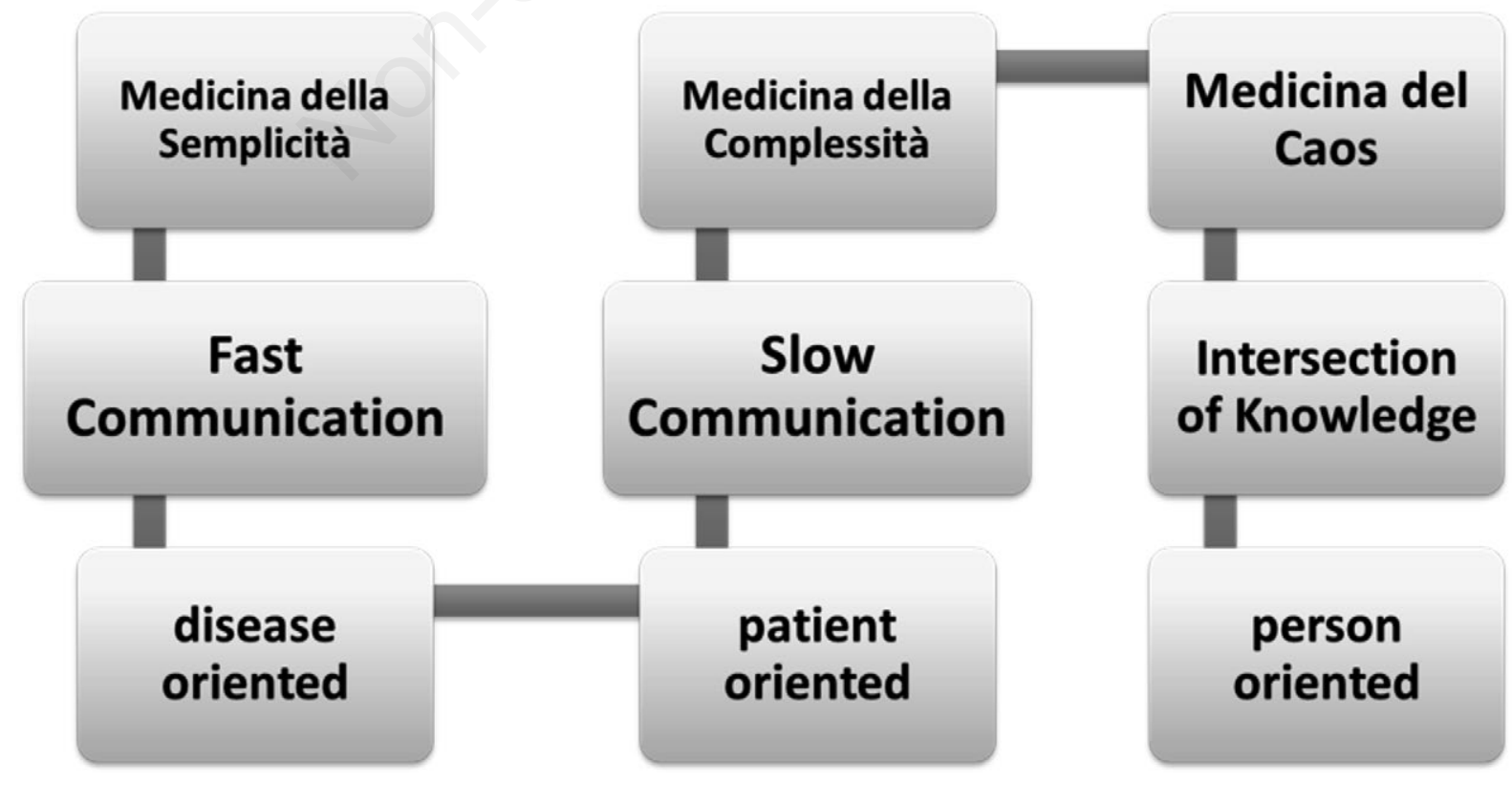

Figura 1 . Il contesto clinico, il registro comunicativo e il metodo clinico. 
sfacente distinzione fra pazienti robusti (Fit), vulnerabili (Vulnerable) e fragili (Frail). Tale caratterizzazione è alla base di processi decisionali sull'appropriatezza clinica e prognostica di strategie diagnostiche e terapeutiche già in atto sia sul campo oncologico geriatrico ${ }^{12}$ che su quello chirurgico cardiovascolare. ${ }^{13}$ La classificazione del paziente in fit, vulnerable o frail deve essere utilizzata dal medico per determinare le effettive possibilità e risorse che il paziente possiede in relazione al suo stato di salute. In quest'ottica la valutazione della fragilità diviene uno strumento da utilizzare nel momento in cui le idee, le preoccupazioni e le aspettative del paziente, cioè il suo illness, non sono in linea con l'oggettività del suo stato di salute. In molti casi la mutata funzionalità del paziente lo porta a non accettare lo stato di cambiamento in atto e quindi rifiutare l'assistenza e la cura. La presa di consapevolezza che deriva dall'essere fit o frail da parte del paziente, è condizione necessaria per progettare una cura personalizzata e per negoziare quegli aspetti che della cura stessa, che vengono meno accettati o mal sopportati dal paziente (Tabella 1).

\section{Terzo ostacolo metodologico: il caregiver e il senso di auto-efficacia}

Un ultimo fattore di ostacolo alla comunicazione, ma non per questo meno importante, è costituito dalla presenza dei familiari del paziente. Nella grande maggioranza dei casi il familiare costituisce una risorsa e in alcuni un ostacolo allarmante. Ovviamente ci si riferisce a quei casi in cui il paziente anziano ha perso la capacità e/o la lucidità cognitiva sufficiente per stare in una relazione diretta con il medico e pertanto tale funzione viene necessariamente mediata dal familiare. Rivestire il ruolo di mediatore per il familiare significa dovere, e sapere, interpretare il mondo del paziente e di conseguenza prendere delle decisioni al suo posto. In questo senso la comunicazione può essere ostacolata dai familiari in virtù del loro senso di autoefficacia nel loro ruolo di caregiver al momento della dimissione. I pochi dati disponibili in letteratura mettono in evidenza che maggiore è il senso di auto efficacia del caregiver e minore è il carico dei problemi fisici, psicologici, sociali e finanziari di cui soffre derivanti dal prendersi cura delle persone anziane. Il senso di auto efficacia è costituito dalle convinzioni circa le capacità che il caregiver pensa di avere, o di non avere, di organizzare ed eseguire le sequenze di azioni necessarie per produrre determinati risultati per la salute del paziente. ${ }^{14}$ Appare chiaro che, nell'applicazione dell'approccio metodologico fin qui descritto diventa importante l'esplorazione, indiretta questa volta, dell'illness del familiare. Vale a dire esplorare quali sono le idee della malattia, quali le preoccupazioni e quali le aspettative che il familiare ha nel rivestire il ruolo di caregiver. Questa tecnica comunicativa si rende necessaria qualora la tendenza del familiare è quella di costruirsi una realtà a volte poco aderente alla situazione oggettiva di salute del paziente. Ciò può essere la conseguenza delle condizioni di affettività ed emotività derivante dai sistemi di attaccamento con il paziente. ${ }^{3}$

\section{Conclusioni}

Parlare di ostacoli alla comunicazione nel paziente anziano è certamente un argomento che comporta molteplici varianti. Possiamo ricordare gli ostacoli derivati dallo stato cognitivo del paziente, dai suoi stati emotivi e dai suoi deficit sensoriali. In termini di abilità comunicative intese come competenza clinica e non come attitudine personale, di fronte a pazienti contestualizzati sempre più nella medicina della complessità e del caos, affrontare il problema con un approccio metodologico aiuta il clinico a progettare un percorso di vita e di cura al paziente in un'ottica di medicina person centered (Figura 2).

Quando il processo comunicativo necessario per costruire la relazione medico paziente, inizia dall'esplorazione dell'illness del paziente (quale idea ha della sua malattia, quali sono le sue preoccupazioni e quali sono le sue attese) e poi si sviluppa lungo la direzione di riconoscere il contesto clinico più appro-

Tabella 1. Principi guida nella comunicazione con il paziente anziano.

\begin{tabular}{ll}
\hline Accogliere le preferenze del paziente & $\begin{array}{l}\text { Registrare l'illness del paziente e della famiglia e definire quali sono gli } \\
\text { obiettivi di cura in base ai desideri riferiti }\end{array}$ \\
\hline Interpretare ed applicare le evidenze cliniche & $\begin{array}{l}\text { Esistono evidenze cliniche certificate? Sono applicabili al paziente? Che } \\
\text { cosa ci si può attendere? }\end{array}$ \\
\hline Stimare la prognosi del paziente (fit, vulnerabile, fragile) & Può essere stimata l'attesa di vita del paziente? \\
\hline Considerare la fattibilità del trattamento & $\begin{array}{l}\text { Il trattamento è compatibile con le condizioni cliniche del paziente e con le } \\
\text { altre terapie? }\end{array}$ \\
\hline Ottimizzare le terapie e definire il piano di vita e di cura & Ricercare, condividere e monitorizzare nel tempo il beneficio netto di salute
\end{tabular}




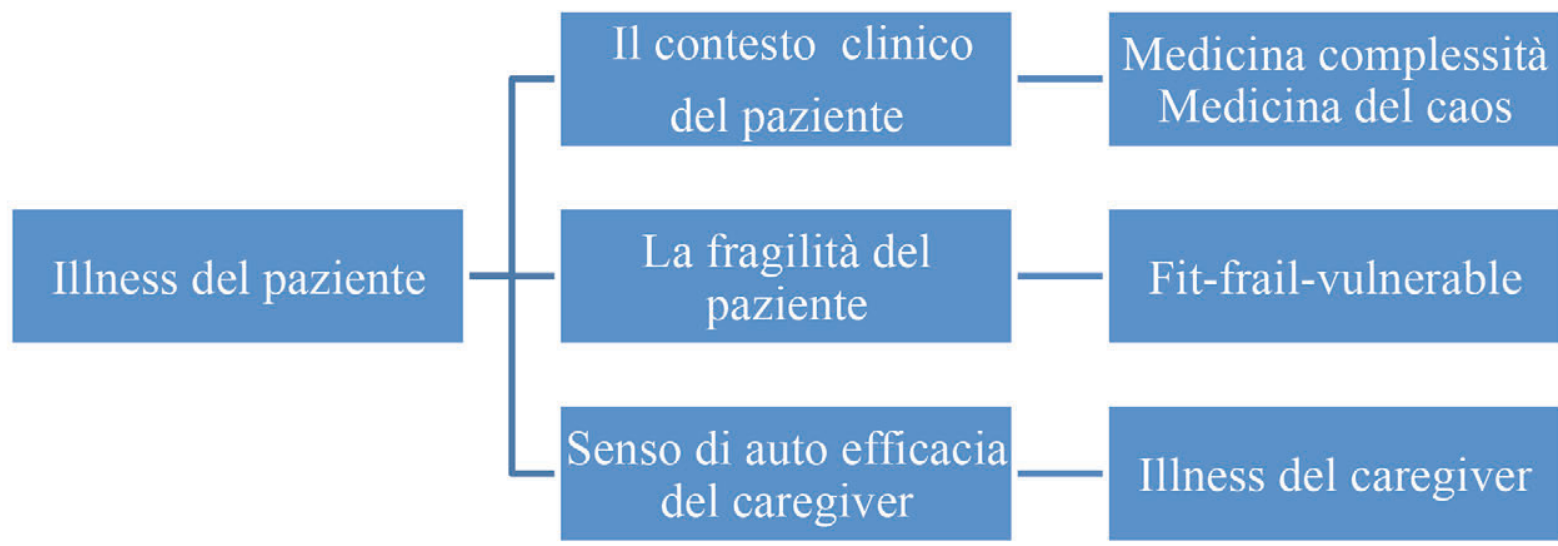

Figura 2. Il progetto di vita e di cura del paziente nelle sue articolazioni.

priato, di definire in modo scientifico la fragilità del paziente stesso e di riconoscere le problematiche relative al senso di autoefficacia del caregiver, è facile pensare di aver ridotto al minimo ulteriori ostacoli comunicativi.

\section{Bibliografia}

1. Richard T, Coulter A, Wicks P. Time to deliever patient centred care. BMJ 2015;350:h530.

2. Felici M, Lenti S. I contesti clinici e comunicativi della medicina. Quaderni Ital J Med 2017;5:e8.

3. Occhini L, Pulerà A, Felici M. Illness e comportamento di malattia. Quaderni Ital J Med 2017;5:46-54.

4. Kurtz F, Snowden DJ. The new dynamics of strategy: sense-making in a complex and complicated world. IBM Syst J 2003;3:462-83

5. Tinetti ME. Potential pitfalls of disease-specific guidelines for patients with multiple conditions. N Engl J Med 351:2870-4.

6. Cagli V. La crisi della diagnosi. Roma: Armando; 2007.

7. Fried LP, Tangen CM, Walston J, et al. Frailty in older adults. Evidence for a phenotype. J Gerontol 2001;56: M146-7.

8. Fried LP, Ferrucci L, Darer J, et al. Untangling the concepts of disability, frailty, and comorbility: implication for improved targeting and care. J Gerontol A Biol Sci Med Sci 2004;59:255-63.

9. Foroni M, Mussi C, Erzili E, Salvioli G. L'anziano fragile e il paziente anziano fragile: problema solo terminologico? G Gerontol 2006;54:255-9.

10. Volpato S, Cavalleri M, Fotini S, Guerra G. Predictive value of the short physical performance battery following hospitalization in older patients. J Gerontol Med Sci 2011;66:89-96.

11. Fried LP, Tangen CM, Wlston J, et al. Frailty in older adults: evidence for a phenotype. J Gerontol Series A Biol Med Sci 2001;56:M146-57.

12. Associazione Italian di Oncologia Medica (AIOM). Linee guida Tumori dell'anziano; 2015. Disponibile a: https://www.aiom.it/category/linee-guida/

13. Heckman GA, Braceland B. Integrating frailty assesment into cardiovascular decision making. Canad J Cardiol 2016;32:139-41.

14. Bandura A. Il senso di autoefficacia. Aspettative su di sé e azione. Trento: Erickson; 1999. 


\title{
L'importanza della capacità di educare all'autogestione delle malattie croniche (self-management) per prevenire l'aggravamento e i ricoveri ripetuti non programmati
}

\author{
Micaela La Regina, ${ }^{1}$ Antonio Greco ${ }^{2}$ \\ ${ }^{1}$ SS Risk Management, ASL 5 Spezzino, La Spezia; ${ }^{2}$ Dipartimento Scienze Mediche, UOC di Geriatria, IRCCS Casa Sollievo \\ della Sofferenza, San Giovanni Rotondo (FG), Italia
}

\section{Introduzione}

In tutti i Paesi si è evidenziata la necessità di sensibilizzare i cittadini sul fenomeno dell'aumento delle patologie croniche. In Europa, una persona su quattro già in età lavorativa ha una malattia cronica (neoplasie, malattie muscoloscheletriche, mal di schiena, cefalea, diabete, bronchite cronica, ipertensione arteriosa, depressione, malattie cardio-vascolari, eccetera), e il mondo del lavoro non è ancora preparato ad affrontare questo fenomeno. Il tasso e l'impatto individuale e sociale delle malattie croniche aumenta ulteriormente nell'età del pensionamento. L'Institute of Medicine (IOM) identifica con sempre maggiore frequenza una discrepanza tra la domanda del bisogno assistenziale nell'ambito della cronicità ed in realtà appare chiaro come l'attuale sistema non possa affrontare questo compito nemmeno anche se spinto a ritmi più sostenuti mentre l'unica via d'uscita è cambiare il sistema. ${ }^{1}$

Il cambiamento è rappresentato dalla responsabilizzazione dei pazienti e dei loro familiari e/o caregiver nella gestione delle malattie croniche, sia da parte delle Aziende Sanitarie, che da parte dei professionisti.

Se l'empowerment è il processo attraverso il quale le persone raggiungono la padronanza delle loro vite ${ }^{2}$ e ancora, un processo educativo finalizzato ad aiutare il paziente a sviluppare la conoscenze, le capacità, le attitudini e il grado di consapevolezza necessari ad assumere responsabilità nelle decisioni che riguar-

Corrispondente: Micaela La Regina, S.S. Risk Management, ASL 5 Spezzino, via Fazio 30, 19121 La Spezia, Italia. Tel.: +39.0187533782.

E-mail: micaela.laregina@asl5.liguria.it

Articolo pubblicato secondo la Creative Commons Attribution NonCommercial 4.0 License (CC BY-NC 4.0).

CCopyright M. La Regina e A. Greco, 2019

Licensee PAGEPress, Italy

QUADERNI - Italian Journal of Medicine 2019; 7(4):63-68 dano la sua salute, ${ }^{3}$ l'espressione concreta e integrata dell'empowerment del paziente è l'autogestione della sua condizione o self management, che dovrebbe costituire un obiettivo cruciale di assistenza per svariate ragioni, quali miglior controllo dei sintomi, mantenimento dell'autonomia funzionale, miglioramento della qualità di vita, riduzione delle complicanze e del ricorso all'ospedale.

Alla base di ciò, tuttavia, vi è uno shift nella relazione paziente-operatore sanitario tradizionale, il passaggio ad un nuovo paradigma in cui i pazienti giocano un ruolo chiaro nel guidare la propria cura $\mathrm{e}$ gli operatori sanitari ne sono partner.

Nel nostro Servizio Sanitario Nazionale sono già previste azioni in tal senso (Tabella 1$){ }^{4}$

Nonostante ciò, i piani di dimissione dall'ospedale e i programmi di follow-up si rivelano spesso carenti, dato che non sempre incentivano l'adozione di rinnovati comportamenti di adeguato follow-up e di autocura.

Nel caso dello scompenso cardiaco, ad esempio, una delle principali cause di ricovero in Medicina Interna, la scarsa o quasi nulla aderenza alla terapia e alle misure dietetiche ed il mancato riconoscimento precoce dei sintomi di aggravamento sono alla base delle riammissioni ripetute non programmate in ospedale.

\section{Il self-management}

Tale termine venne usato per la prima volta oltre 40 anni fa da Thomas Creer per indicare la partecipazione attiva dei pazienti ai loro trattamenti. Oggi il termine autogestione è ampiamente usato ed è descritto da una varietà di definizioni e concettualizzazioni, che contribuiscono a una mancanza di chiarezza e di letteratura.

A livello generale, l'autogestione è definita come la gestione quotidiana delle condizioni di salute di individui nel corso di una malattia cronica.

Sebbene il termine autogestione sia spesso utilizzato in modo intercambiabile con termini come cura di sé, autoregolamentazione, educazione del paziente e counseling del paziente, l'autogestione si è evoluta 
oltre la pratica di fornire semplicemente informazioni e aumentare la conoscenza del paziente. Sebbene i concetti siano correlati, l'autosufficienza è interpretata da molti autori come l'insieme di quei comportamenti svolti da persone sane per prevenire le malattie, piuttosto che limitarsi a gestire la malattia esistente.

L'autoregolamentazione è più lontana, riferita alla capacità di controllare e gestire pensieri, emozioni o comportamenti.

Le descrizioni concettuali e teoriche dell'autogestione con riferimento a componenti, processi e risultati si sono ampliate e si sono evolute dal 1980, quando Corbin e Strauss ${ }^{5}$ hanno identificato 3 serie di compiti associati al lavoro di convivenza con una malattia cronica, quali: i) gestione medica della condizione; ii) gestione del comportamento; e iii) gestione emotiva.

L'autogestione è anche uno dei 6 elementi essenziali identificati nel Chronic Care Model. Tale modello mira a migliorare la qualità e i risultati concentrandosi su cambiamenti a livello di sistema che possono avere un impatto sui fattori relativi al paziente e correlati agli erogatori di assistenza e viene sempre più utilizzato come framework per l'identificazione di elementi del sistema che si ritiene influenzino i risultati della malattia cronica.

L'autogestione di una malattia cronica non esiste in se, ma piuttosto nel contesto di altre persone e influenze. Fondamentali per il suo successo sono le relazioni tra i pazienti e i loro partner in ambito sanitario (principalmente gli infermieri), amici, comunità, e familiari.

La famiglia è un'importante fonte di sostegno per le persone con malattie croniche. Gli individui con livelli più alti di sostegno familiare hanno - è stato dimostrato - una maggiore aderenza all'autogestione e un migliore controllo sulle loro condizioni. Questo è particolarmente importante nell'autogestione a lungo termine dove l'enfasi sulla famiglia sull'autosufficienza e sul successo personale, coesione familiare, e risposte attente ai sintomi sono state associate a migliori risultati per il paziente. La ricerca sull'autogestione familiare e comunitaria ha identificato fattori di rischio e fattori protettivi in grado di influenzare i risultati, nonché potenziali obiettivi di intervento. ${ }^{6}$
Molte condizioni croniche, come le malattie cardiache, il diabete e l'artrite, sebbene uniche nelle loro caratteristiche e richieste, condividono aspetti comuni nella loro gestione. Questi includono: l'affrontare i sintomi e la disabilità, il monitoraggio degli indicatori fisici, la gestione di complessi trattamenti farmacologici, il mantenimento di adeguati livelli di nutrizione, dieta ed esercizio fisico, l'adeguamento alle esigenze psicologiche e sociali, compresi i difficili adattamenti dello stile di vita.

L'identificazione e l'elaborazione di strategie comuni incentrate sul paziente per affrontare queste sfide è al centro del campo dell'autogestione. Indipendentemente dalla condizione cronica, lo sviluppo di un insieme generico di abilità si è dimostrato efficace nel permettere alle persone di gestire efficacemente la loro malattia e migliorare i risultati sanitari.

Altrettanto importante è l'esigenza di sviluppare modelli più sofisticati di autogestione, adattati alle varie condizioni e situazioni di salute.

\section{Digital Health e self-management}

La diffusione degli strumenti digitali (PC, smartphone, tablet, ecc.) con i loro applicativi (internet, apps, ecc.) e l'avvento dei social network ha influenzato e continua ad influenzare il mondo sanitario, compreso il self-management delle malattie croniche.

Le nuove tecnologie consentono molti più trattamenti e opzioni di gestione delle malattie croniche sia per i medici che per i pazienti. Tali soluzioni si estendono a tutto lo spettro di cure e includono diagnosi e monitoraggio, rilevamento precoce del rischio, trattamento e riabilitazione, ma anche fornitura di feedback e allarmi e strategie educazionali e motivazionali che facilitano cambiamenti nei comportamenti disfunzionali o mantenimento di uno stile di vita sano. ${ }^{7}$

Dispositivi biomedici come i glucometri, utilizzati per misurare i livelli di zucchero nel sangue, ora devono essere interconnessi con altri dispositivi medici e sistemi di cartelle cliniche elettroniche. Le informazioni prodotte da tali dispositivi sono più utili se considerate nel contesto generale della cura del paziente

Tabella 1. Dall'educazione al self management e oltre.

[...] Si tratta di un percorso, strutturato e sistematico che, partendo dall'informazione-educazione sugli stili di vita utili a contrastare il malessere e l'evoluzione della patologia, deve progressivamente fornire una serie di competenze e di abilità al paziente ed ai Caregiver orientate verso:

- la capacità di fare fronte alla malattia;

- il mantenimento dello stato di salute (self care maintenance); $e$

- progressivamente, verso la vera e propria autogestione della patologia (self care management).Rispetto all'efficacia del processo educativo va segnalata la tendenza dei sistemi più avanzati a desanitarizzare quest'area, evitando di perseguire gli eccessi specialistici della conoscenza e ricorrendo ad attori potenzialmente più empatici e più comunicativi. Fondamentale, quindi, in quest'area il ruolo dei pazienti-esperti, capaci di condividere e trasmettere anche il proprio vissuto, così come importante è il ruolo del volontariato esperto, annoverato nell'ampia area di esperienze che fa capo all'autogestione promossa attraverso personale laico, non professionista (Lay-led Self Management) [...]

Fonte: Ministero della Salute, Piano Nazionale della Cronicità, Accordo tra lo Stato, le Regioni e le Province Autonome di Trento e di Bolzano del 15 settembre 2016. 
insieme ad altri segni vitali, ad esempio la pressione sanguigna, la temperatura e il peso. Lo sviluppo di dispositivi medicali contestuali intelligenti supporterà diagnosi e monitoraggio più accurati, supporto per l'autogestione e la cura di sé una volta che un paziente ritorna alla comunità.

Lo sviluppo di tali dispositivi è stato recentemente applicato anche a sottogruppi di popolazione di età più avanzata teoricamente meno pronti a cogliere ed utilizzare le opportunità dello strumento informatico. In una recente esperienza condotta nel nostro Ospedale si è collaudato un sistema di controllo a distanza della glicemia, della pressione corporea e degli stili di vita (tempo dedicato all'attività fisica riscontrando una buona accettabilità del sistema da parte del paziente ed un controllo più efficace dei valori di pressione arteriosa e di glicemia). Il sistema basato su di una app in grado di girare su smartphone (Android) è collegato con sistema near field communication (NFC) che collega il telefono ad uno sfigmomanometro ed ad un glucometro. $^{8}$

Lo stetoscopio che può essere collegato a un telefono cellulare consente ai pazienti di monitorare la propria salute polmonare.

Altri dispositivi biomedici emergenti che supportano una migliore gestione delle malattie croniche includono un sistema di riabilitazione per pazienti con ictus che usano feedback vibrotattile, un nuovo sistema di analisi del movimento facciale per paresi facciale, un'esplorazione degli effetti dell'esercizio per dialisi e malati di cancro e l'influenza di ambiente su quelli con demenza e malattia coronarica.

Numerosi sistemi di information and communication technology sono stati validi come suopporto alle activities of dailiy living del paziente anziano con demenza ed in particolare anche con le funzioni di memory trainer e tele- riabilitazione cognitiva. Il progetto Alzheimer patient's home rehabilitation by a Virtual Personal Trainer-based Unique Information System Monitoring (ALTRUISM) nell'ambito del Bando Aiuti a Sostegno dei Partenariati Regionali per l'Innovazione, Regione Puglia 2017 ha dimostrato, su trenta soggetti affetti da malattia di Alzheimer, un rallentamento della progressione di malattia. ${ }^{9}$

Gli smartphone e altri dispositivi mobili ora hanno la capacità di un personal computer e, se accoppiati anche con una connessione Internet di base, possono consentire il tele-monitoraggio, cioè il trasferimento di dati fisiologici come pressione sanguigna, peso, dettagli elettrocardiografici e saturazione di ossigeno attraverso il cavo telefonico o digitale da casa al fornitore di servizi sanitari. Un recente Cochrane Review ha confermato il valore del tele-monitoraggio non invasivo per le persone con insufficienza cardiaca nel ridurre la mortalità del $20 \%$ e i ricoveri relativi allo scompenso cardiaco del $29 \%$. Combinato con un sup- porto telefonico strutturato, ha ridotto la mortalità per tutte le cause del 13\% e nel processo ha fornito un'esperienza accettabile per i pazienti. ${ }^{10}$

Sempre basate sugli smartphone e altri dispositivi mobili anche le apps sono sempre più utilizzate per poter assistere i pazienti cronici nell'autogestione. Tuttavia, una recente revisione che ha passato in rassegna le evidenze relative all'efficacia, all'usabilità e alla valutazione delle app volte ad assistere i pazienti nell'autogestione di 11 patologie differenti ha promosso solo le app per il diabete e i benefici rilevati erano di importanza clinica marginale, non su endpoint clinici difficili, come mortalità o ospedalizzazioni. Questo non esclude le loro potenzialità, solo richiama alla necessità di progettarle e sperimentarle per conseguire benefici clinici, infatti solo una minoranza di studi faceva riferimento esplicitamente l'utilizzo di teorie del cambiamento comportamentale per sostenere l'intervento dell'app.

Da notare infine che i pazienti hanno iniziato a utilizzare sempre più il Web come strumento di comunicazione, anziché come semplice distributore di informazioni e ciò ne amplifica l'utilità potenziale nell'autogestione. Sebbene gli over 65 , più spesso affetti da patologie croniche, rimangano fortemente collegati alle fonti di assistenza medica offline, l'uso dei consigli dei social network tra di essi è in crescita. Circa 1 su 3 (34\%) di essi accedono a siti di social networking come Facebook e LinkedIn e circa 1 su 5 (20\%) di questi utenti contribuisce regolarmente a questi siti taggando, categorizzando o commentando i contenuti sanitari/medici online. In particolare, sono disposti a condividere le informazioni sulla cura di sé all'interno di determinati social network allo scopo di fornire e ricevere informazioni di autogestione specifiche per la malattia. L'evoluzione delle comunità di e-patient ha portato a maggiori opportunità di acquisizione di conoscenze e supporto sociale, portando a una migliore qualità della vita correlata alla malattia.

Tradizionalmente, gli esperti di salute pubblica hanno fornito informazioni sulle malattie croniche in forma statica con materiali scritti e audio online (Web 1.0). L'eHealth ha mostrato di poter migliorare i risultati di salute in modo anche economicamente conveniente. La rapida crescita nell'adozione delle tecnologie Web 2.0, come documentato sopra, suggerisce che gli interventi Internet partecipativi possono aiutare gli individui più con malattie croniche a diventare attivamente impegnati nella propria assistenza sanitaria. Aggiustando i risultati per l'età, l'istruzione e il tipo di accesso a Internet, è stato dimostrato che vivere con una malattia cronica aumenta la probabilità di contribuire o di visualizzare contenuti di salute generati dagli utenti come post di blog, esami ospedalieri o medici e podcast. Inoltre, $\mathrm{i}$ forum di discussione online forniscono uno spazio ad accesso aperto per i pazienti con malattie croniche 
per lo scambio di informazioni sulla patologia e su come controllare le riacutizzazioni della malattia. infine, le evidenze disponibili mostrano che i gruppi di auto-assistenza online possono aumentare il capitale sociale in modi che non indeboliscono e, anzi potrebbero in alcuni casi rafforzare le connessioni iperpersonali tra pazienti e operatori sanitari.

Questo è solo un esempio di come electronic Health (eHealth) e mobile Health (mHealth) possono migliorare i risultati di salute, la qualità della vita e il benessere e facilitare l'empowerment e l'impegno del paziente funzionale. La mobile Health, utilizzata con dispositivi come impianti e sensori biomedici, ha il potenziale per essere una tecnologia dirompente che potrebbe superare molte delle limitazioni associate al trattamento tradizionale, limitato e costoso di molte patologie croniche. Il modello consentirebbe di adeguare la gestione delle malattie croniche alle esigenze di ogni singolo individuo.

\section{Il ruolo della robotica}

L'impiego di strumenti informatici associati a componenti sensoriali è stato ulteriormente migliorato dall'impiego di robot assistivi. Il robot unisce all'integrazione di queste due componenti la percezione di un unicum percepito come una sorta di compagno che quindi favorisce potenzialmente una miglior integrazione tra assistente/assitito.

In questo ambito l'UOC di Geriatria di San Giovanni Rotondo ha sperimentato nell'ambito di diversi progetti Europei tre diversi robot.

Nella prima esperienza il Robot MARIO (Managing Active and healthy aging with use of caRing servIce robots, Horizon 2020, PHC-21) è stato usato nell'UOC di Geriatria di Casa Sollievo della Sofferenza per prevenire l'isolamento sociale e rallentare il deficit cognitivo dell'anziano fragile.

Un numeroso gruppo di anziani ha dimostrato un'elevata accettabilità del robot, una significativa riduzione della depressione nei soggetti con Alzheimer e dello stress nei loro care giver. ${ }^{11}$

Una seconda esperienza, tuttora in corso, basata sulla metodologia della Agile Co-Creation in cui sviluppatori ed end user lavorano insieme allo sviluppo del robot è dedicata alla prevenzione del rischio di cadute. Il Progetto denominato Agile CoCreation of Robots for Ageing, su bando Horizon 2020, PM-14, della Comunità Europea è realizzato mediante la creazione di un robot in grado di favorire la mobilità dell'anziano prevendo disturbi della statica e conseguentemente il rischio di cadute. Il Robot denominato ASTRO integra una componente motoria ad una sensoriale con cui è in grado di registrare il tono muscolare degli arti eseguendo un monitoraggio di eventuali improvvisi deficit di forza in grado di generare la caduta.
Associato ad ASTRO è stato realizzato un altro Robot denominato Buddy sostanzialmente simile ad un piccolo cagnolino in grado di stimolare l'anziano a compiere movimenti e giochi in grado di favorire il mantenimento di schemi motori e comportamentali. ${ }^{12}$

\section{I benefici del self-management}

Due dei programmi di autogestione di maggior successo e più noti sono l'Arthritis Self-Management Program (ASMP) e il Diabetes Self-Management Program (DSMP), sviluppati con NINR e supporto del NIH da 2 pionieri nel campo. ${ }^{13}$

L'ASMP hanno mostrato nei soggetti coinvolti riduzione del dolore, maggiore autoefficacia percepita e maggiore qualità di vita, meno visite mediche $\mathrm{e}$, in alcuni casi, ridotta disabilità. Tali risultati sono persistiti per almeno 4 anni dopo la partecipazione iniziale al programma. L'ASMP è stato il precursore per lo sviluppo dello Stanford Chronic Disease Self-Management Program (CDSMP) che è stato adottato negli Stati Uniti, in Canada, Australia, Nuova Zelanda, Regno Unito, e in altri paesi e tradotto in numerose lingue per l'uso in tutto il mondo.

I programmi di autogestione continuano a essere sviluppati, valutati e implementati con successo per numerose malattie e condizioni croniche.

Organismi come la Cochrane Collaboration hanno condotto analisi basate sull'evidenza di varie strategie di autogestione, trovando merito in programmi focalizzati su argomenti quali terapia anticoagulante, asma, disturbo bipolare, diabete, ecc.

La capacità di autogestire le condizioni croniche, tuttavia, è direttamente influenzata da fattori legati alla propria comunità e all'ambiente domestico e alle risorse. Le disparità demografiche contribuiscono alla formazione di alcune barriere ambientali che possono risultare in condizioni croniche. Ad esempio, alcune popolazioni hanno scarso accesso a strutture per il fitness e ricreative o a negozi di alimentari che vendono frutta e verdura fresca. Lo stesso dicasi per l'ambiente domestico e cittadino. Da qui la necessità di affiancare interventi sociali o domestici per sostenere l'autogestione delle malattie croniche.

\section{Self-management e utilizzazione delle risorse sanitarie}

Un fattore chiave dell'interesse attuale per l'autogestione è il suo potenziale contributo all'erogazione di un'assistenza sanitaria efficiente, attraverso il miglioramento delle attività di prevenzione e la riduzione della dipendenza dai servizi di assistenza sanitaria tradizionale. Tuttavia, la portata del contributo dell'autogestione alla gestione della domanda non è chiara. 
Rapporti positivi sugli impatti del supporto di autogestione sull'utilizzo delle cure sanitarie non sono sempre stati replicati, e alcuni interventi di autogestione potrebbero aumentare la domanda. Se i pazienti non sono pronti o sufficientemente preparati per i nuovi ruoli, possono subire conseguenze negative. Aumentare il ruolo dei pazienti nella gestione delle condizioni a lungo termine, riducendo al contempo l'accesso ai servizi formali, può provocare ansia, difficoltà di gestione e riduzione della qualità delle cure. Raggiungere risparmi sui costi riducendo significativamente la qualità della vita dei pazienti sarebbe un risultato negativo per i servizi sanitari e i pazienti. ${ }^{14}$

La domanda è quindi: vi sono modelli di supporto all'autogestione associati a significative riduzioni nell'utilizzo dei servizi sanitari senza compromettere i risultati, tra i pazienti con condizioni a lungo termine?

Una recente revisione ${ }^{15}$ che ha incluso 184 studi sul self-management in una grande varietà di patologie croniche ha provato a rispondere a questa domanda. I dati raccolti hanno evidenziato che gli interventi di supporto all'autogestione ${ }^{16,17}$ possono ridurre l'utilizzo dei servizi sanitari senza compromettere gli esiti della salute dei pazienti, possono ridurre l'uso dell'ospedale e i costi totali, sebbene gli effetti siano generalmente limitati. L'evidenza di riduzioni significative nell'utilizzo è più evidente e robusta per gli interventi nei disturbi respiratori e cardiovascolari.

Fra le diverse tipologie di interventi di supporto all'autogestione delle malattie croniche, il coinvolgimento della famiglia nell'autogestione ha dimostrato di migliorare i tassi di riammissione in ospedale e di accesso al pronto soccorso e gli interventi di transitional care (comprendenti l'istruzione sull'autogestione, la pianificazione delle dimissioni, il follow-up strutturato e il coordinamento tra i diversi operatori sanitari) una percentuale inferiore di accessi in pronto soccorso a 3 mesi, un tasso inferiore di riammissioni a 3, 6, 12 e 18 mesi, una media inferiore dei giorni di riammissione a 3, 6, 12 e 18 mesi, una mortalità inferiore a 36,12 e 18 mesi dopo la dimissione, ragion per cui tali interventi andrebbero inclusi nella riorganizzazione dei servizi sanitari.

\section{Conclusioni}

Dal momento che le patologie croniche rappresentano ormai una priorità per la salute pubblica, l'autogestione continuerà a crescere come approccio cruciale alla gestione di queste condizioni, alla prevenzione delle complicanze e alla promozione del benessere.

Esistono evidenze promettenti, come delineate in questo articolo, circa l'efficacia e efficienza dei programmi di intervento di autogestione e potenzialità nell'ambito della salute digitale..$^{18,19}$
L'autogestione ha un valore particolare in quanto rappresenta una fusione degli obiettivi del paziente, della famiglia, del clinico, della comunità e del sistema sanitario. A tale riguardo, l'autogestione va al di là della tradizionale gestione delle malattie incorporando il più ampio concetto di prevenzione, sottolineando l'idea che coloro che sono malati cronici hanno ancora bisogno di interventi preventivi per promuovere il benessere e mitigare l'ulteriore deterioramento della salute. ${ }^{20}$ Quindi, se si considera la natura dell'autogestione in tutti i suoi elementi e le sue caratteristiche pratiche, non è solo un approccio logico alla salute e all'assistenza sanitaria, ma è anche un modo ottimale per affrontare le condizioni croniche come un problema di salute pubblica e di equità sociale.

\section{Bibliografia}

1. Adams S, Smith PK, Allan PF, et al. Systematic review of the chronic care model in chronic obstructive pulmonary disease prevention and management. Arch Intern Med 2007;167:551-61.

2. Rappaport J. Terms of empowerment/exemplars of prevention: Toward a theory for community psychology. Am J Commun Psychol 1987;2:121-48.

3. Feste C, Anderson RM. Empowerment from physolophy to practice. Patient Educ Cons 1995;26:139-44.

4. Mistero della Salute. Piano Nazionale della Cronicità. Disponibile su: http://www.salute.gov.it/imgs/C_17_pubblicazioni_2584_allegato.pdf

5. Corbin J Strauss A. Grounded theory research: procedures, canons, and evaluative criteria. Qual Sociol 1990;13:3-22.

6. Pathways Project. Employment \& Chronic Diseases, New Pathways In Europe, Pathways Project-Participation To Healthy Workplaces And inclusive Strategies in the Work Sector; March 2019. Available from: https://www.path-ways.eu/

7. Grady PA, Grough LL. Self-management: a comprehensive approach to management of chronic conditions. Am J Public Health 2014;104:e25-31.

8. Sancarlo D, D'Onofrio G, Matera A, et al. Implementation of a solution for the remote monitoring of subjects affected of metabolic disease: The Metabolink Project. In: Cavallo F, et al., eds. Ambient Assisted Living: Italian Forum. Milano: Springer; 2016. pp 191-6.

9. D’Onofrio G. Information and communication technologies for the activities of daily living in older patients with dementia: a systematic review. J Alzheim Dis 2017;57:927-35.

10. Corazza G, Nardi R. Prime esperienze di governo clinico-assistenziale. Lo scompenso cardiaco: il progetto OSCAR (Ottimizzazione della terapia e della gestione dello Scompenso cardiaco nell'Anziano con Ricoveri ripetuti). Nursing Manage Inferm 2003;9:37-47.

11. D'Onofrio G. Assistive robots for socialization in elderly people: results pertaining to the needs of the users. Aging Clin Exp Res 2018 [Epub ahead of print].

12. D'Onofrio G, Fiorini L, Hoshino H, et al. Agile Co-Creation for Robots and Aging (ACCRA) Project: new tech- 
nological solutions for older people. Eur Geriatr Med 2018;9:795-800.

13. Lorig K. Chronic disease self-management program: insights from the eye of the storm. Front Public Health 2014;2:253.

14. Turner RM, Ma Q, Lorig K, et al. Evaluation of a diabetes self-management program: claims analysis on comorbid illnesses, health care utilization, and cost. J Med Internet Res 2018;22:e207.

15. Stellefson M, Chaney B, Barry AE, et al. Web 2.0 Chronic disease self-management for older adults: a systematic review. J Med Internet Res 2013;15:e35.

16. Scott IA, Scuffham P, Gupta D, et al. Going digital: a narrative overview of the effects, quality and utility of mobile apps in chronic disease self-management. Aust Health Rev 2018 [Epub ahead of print].

17. Lewis J, Ray P, Liaw ST. Recent worldwide develop- ments in eHealth and mHealth to more effectively manage cancer and other chronic diseases - a systematic review. Yearb Med Inform 2016;1:93-108.

18. Panagioti M, Richardson G, Small N, et al. Self-management support interventions to reduce health care utilisation without compromising outcomes: a systematic review and meta-analysis. BMC Health Serv Res 2014;14:356.

19. McBain H, Shipley M, Newman S. The impact of selfmonitoring in chronic illness on healthcare utilisation: a systematic review of reviews. BMC Health Serv Res 2015; 15:565.

20. Le Berre M, Maimon G, Sourial N, et al. Impact of transitional care services for chronically ill older patients: a systematic evidence review. J Am Ger Soc 2017;65: 1597-608. 


\title{
Le cure di fine vita negli anziani: il diritto di morire bene
}

\author{
Luigi Lusiani, ${ }^{1}$ Claudio Santini ${ }^{2}$ \\ ${ }^{1}$ ex Direttore SOC di Medicina Interna, PO di Castelfranco Veneto (TV), AULSS 2 Veneto, Basiliano (UD); ${ }^{2}$ Direttore UOC \\ di Medicina Interna, Ospedale Vannini, Roma, Italia
}

\section{Morire è naturale}

Le persone anziane muoiono, naturalmente.

Definire quando una persona diventa anziana è problematico e arbitrario, ma, a prescindere da ciò, si può dire che nessuno ha difficoltà ad accettare questa asserzione iniziale, anche se, in realtà, ciascuno avverte una qualche istintiva resistenza interiore se si parla della propria morte.

Oggi si parla con disinvoltura di superamento della morte attraverso la crioconservazione del soma (o di parte di esso), la manipolazione genetica, il potenziamento del corpo e della mente con estensioni o sostituzioni protesiche di vario tipo che ci trasformerebbero in simbionti o in una nuova specie di homo technologicus destinato a portare ad estinzione l'homo sapiens; se ne parla, come destino che ci attende, senza che ciò abbia una serie base scientifica per costruire ipotesi plausibili al di là di annunci visionari. ${ }^{1,2}$ Tuttavia, dobbiamo per ora ammettere che la morte resta parte della nostra prospettiva biologica, come è stato per tutti i nostri simili che ci hanno preceduto, e quindi della nostra natura. Qualcosa con cui fare i conti, senza esorcismi. ${ }^{3}$

Eppure, paradossalmente, la morte come fatto naturale non trova un chiaro riscontro, né una appropriata traduzione in ambito medico ospedaliero. Prova ne è che la categoria morte naturale non è contemplata nelle classificazioni delle cause di morte nei sistemi ICD9-CM e ICD10. Da ciò risulta che in ospedale si muore ufficialmente e immancabilmente a causa di qualche malattia.

Per quanto sia innegabile il fatto che, se una per-

Corrispondente: Luigi Lusiani, ex Direttore SOC di Medicina Interna, PO di Castelfranco Veneto (TV), AULSS 2 Veneto, via Portatrici carniche 2, 33031 Basiliano (UD), Italia. E-mail: g.lusiani52@gmail.com

Articolo pubblicato secondo la Creative Commons Attribution NonCommercial 4.0 License (CC BY-NC 4.0).

CCopyright L. Lusiani e C. Santini, 2019

Licensee PAGEPress, Italy

QUADERNI - Italian Journal of Medicine 2019; 7(4):69-74 sona finisce in ospedale negli ultimi giorni di vita, ciò avviene perchè la stessa è portatrice di uno o di plurimi stati morbosi, va anche detto che sussiste una differenza sostanziale tra chi muore a causa di, e chi muore in presenza di una data condizione. La mancata distinzione non è un fatto solamente formale, né è irrilevante dal punto di vista clinico, perché da un lato produce distorsioni di ordine epidemiologico, dall'altro alimenta la crescente medicalizzazione delle fasi terminali della vita dei malati cronici e della loro morte, cui stiamo assistendo.

Sul piano epidemiologico, si creano le premesse per dimostrare erroneamente che la medicina sta fallendo nel prevenire la mortalità determinata da specifiche condizioni morbose, che tipicamente si presentano negli stati terminali, senza per questo essere veramente responsabili di un esito evitabile (l'embolia polmonare costituisce un chiaro esempio di ciò). Sul piano clinico, quello che più direttamente riguarda la pratica medica, si lascia intendere che è sempre possibile scongiurare o almeno dilazionare la morte (vista come un fallimento, per definizione) intervenendo tecnologicamente sulle sue cause. Nella mentalità garantista che pervade la nostra cultura, se ciò è possibile, è anche doveroso, ed anzi dovuto. In realtà si tratta di un malinteso micidialmente nocivo, perché tende a giustificare a priori l'oltranzismo medico, e al contempo a favorire il fatto che l'ospedalizzazione in fase terminale diventi apportatrice ai morenti di sofferenze aggiuntive, sostenute da cure indebite e futili.

Recuperare, là dove è possibile ed ha senso, il concetto di naturalità del morire è dunque un compito di ordine culturale, che investe tutta la nostra società nel suo insieme e nelle sue diverse componenti, ma in particolare la classe medica, in quanto essa è chiamata a presidiare questo passaggio vitale.

Dobbiamo ammettere che la morte resta parte della nostra prospettiva biologica, come è stato per tutti i nostri simili che ci hanno preceduto, e quindi della nostra natura. Qualcosa con cui fare $i$ conti, senza esorcismi. Recuperare, là dove è possibile ed ha senso, il concetto di naturalità del morire è un compito di ordine culturale, che investe tutta la nostra so- 
cietà nel suo insieme e nelle sue diverse componenti, ma in particolare la classe medica, in quanto essa è chiamata a presidiare questo passaggio vitale.

\section{Morire bene è un diritto}

Morire bene può suonare contraddittorio, in quanto salda qualcosa di intrinsecamente ripugnante, come la morte, con una categoria desiderabile come il bene. $\mathrm{Ma}$ in realtà è importante che venga presentato come un diritto per i malati terminali, e come un dovere per i medici nei loro confronti, e parte importante dei programmi di salute.

Anche se, quando si parla di salute, nessuno può sentirsi autorizzato a rivendicare per sé o per gli altri il diritto ad un risultato (il morire bene, in questo caso), va affermato che a tutti i malati alla fine della vita devono essere garantite cure appropriate al loro stato. Si tratta di cure diverse da quelle che si rivolgono ai malati in fase acuta, che devono ispirarsi a paradigmi che non sono più la ricerca etiologica e la guarigione, bensì il controllo dei sintomi in quanto tali e l'accompagnamento psicologico e spirituale (comfort medicine). ${ }^{4,5}$

Affinchè il morire bene possa essere perseguito, in primo luogo è necessario che la morte, una volta giudicata - sulla base di discernimento clinico - come ineluttabile esito della traiettoria della malattia in atto, essa venga esplicitamente contemplata ed anzi annunciata, e assunta nella sua valenza clinica. Questo passaggio non è per nulla scontato, né facile, perché impegna fortemente sul piano emotivo in primo luogo il paziente, ma anche medici e familiari, e presuppone un livello comunicativo non ordinario.

Ciò che ordinariamente avviene, invece, è che la prospettiva della morte venga taciuta, per una forma di rispetto e per debolezza di pensiero, che nei medici si instauri una rassegnata inerzia come di fronte ad un fallimento irreparabile, e che - quel che è peggio - vengano mantenute le abituali opzioni diagnostico-terapeutiche, come se si dovesse ancora scongiurare qualcosa; senza tener conto che alla fine dell'esistenza le cure abituali tendono a diventare sproporzionate e addirittura dannose, quando quello che conta di più diventa la qualità residua e non necessariamente la quantità ulteriore di vita.

Nei malati ricoverati in ospedale e ormai giunti alla loro fase terminale, misurabile nell'ordine delle settimane e non di più, gli internisti devono essere capaci di proporre e gestire la comfort medicine nel loro reparto, come medicina rivolta scientemente ai sintomi disturbanti, e solo a quelli (dolore, dispnea, astenia, anoressia, disturbi dell'alvo, nausea/vomito, ansietà, depressione, delirium, agitazione, secchezza delle fauci, eccessive secrezioni respiratorie e quant'altro sia soggettivamente disturbante a prescindere dalla gravità), e questo in maniera proattiva (non solo interventi su chiamata e al bisogno), trascurando ogni intento diagnostico e il controllo accanito dei parametri vitali, garantendo invece sostegno sul piano psicologico e spirituale; il tutto dopo aver pianificato ogni programma di concerto con i malati (se competenti) e l'ambiente dei familiari care-giver, attraverso passaggi comunicativi non sbrigativi e necessariamente reiterati. Deve essere chiaro che le cure di fine vita non sono finalizzate al prolungamento della vita residua, ma al benessere ancora possibile, al sollievo dai disturbi e all'accompagnamento ad una morte di qualità, senza abbandoni né rinunce: non si tratta di rinunciare a cure, ma di orientarsi al comfort del paziente.

Conviene non confondere tutto ciò con le cure palliative vere e proprie: queste ultime abbracciano un arco di tempo maggiore se sono iniziate (come è raccomandato) in maniera tempestiva, possono essere simultanee rispetto ai trattamenti curativi (come è raccomandato nei neoplastici avanzati), richiedono personale dedicato che garantisca continuità fra le cure territoriali e quelle ospedaliere e interventi a scavalco. Le cure di fine vita ospedaliera devono saldarsi con le cure palliative già istituite, ma gli internisti devono essere in grado di gestire in proprio la comfort medicine intraospedaliera, con capacità di iniziativa anche nelle situazioni carenti.

A tutti i malati alla fine della vita devono essere garantite cure diverse da quelle che si rivolgono ai malati in fase acuta, che devono ispirarsi a paradigmi che non sono più la ricerca etiologica e la guarigione, bensì il controllo dei sintomi in quanto tali $e$ l'accompagnamento psicologico e spirituale (comfort medicine).

\section{La responsabilità degli internisti}

Gli studi dimostrano come, nel nostro come in altri paesi, i pazienti cronici espressamente interpellati esprimano la preferenza di concludere la loro esistenza tra le mura domestiche e i conforti familiari. Ma in realtà la maggior parte di essi decede in ospedale, dove viene ricoverata quando le condizioni cliniche si deteriorano rendendo le cure domiciliari impraticabili, ma altre volte per scelta dei familiari, impreparati, spaventati o semplicemente confusi all'idea di gestire il fine vita dei loro anziani a casa. ${ }^{6,7}$

I dati italiani relativi ai ricoveri ospedalieri mostrano come nel 2014, su un totale di 198.156 decessi registrati nelle SDO, 102.498 (par al 51,7\%) sono intercorsi nei reparti internistici o geriatrici; i decessi registrati specificamente nelle Medicine Interne sono 
risultati più numerosi di quelli registrati in tutti gli altri reparti singolarmente considerati, secondo un fattore di 2,5 rispetto alle Terapie Intensive, 5,6 rispetto alle Geriatrie, 7,9 rispetto alle Cardiologie, 2,5 rispetto a tutte le rimanenti specialità cumulativamente conteggiate [dati confidenziali, analizzati e messi a disposizione da G. Mathieu]. Non è da attendersi che questi numeri possano variare in termini assoluti in un futuro prossimo, perché, anche nella prospettiva che presto proliferino le strutture alternative all'ospedale per i pazienti terminali (cfr. Hospice e altre forme di servizi domiciliari), l'aumento della popolazione anziana tenderà a controbilanciarne l'effetto favorevole. Dunque è necessario che gli internisti, già ora coinvolti per forza di cose, coltivino e migliorino la loro professionalità nelle cure di fine vita, come parte integrante $\mathrm{e}$ qualitativamente molto importante del loro lavoro.

Tuttavia bisogna ammettere che le cure di fine vita rappresentano una necessità non ancora pienamente soddisfatta in ospedale, soprattutto per la mancanza di formazione pre-laurea e post-laurea indirizzata in questo senso. Il ritardo con cui il mondo Accademico e le Facoltà di Medicina stanno affrontando il problema viene compensato con percorsi formativi suppletivi (cfr. Master e altri corsi professionalizzanti) in attesa di iniziative più strutturate e robuste per il futuro. Quand'anche le Cure Palliative venissero via via organizzate all'interno degli ospedali e nei territori collegati con personale specificamente dedicato (cosa di per sé auspicabile), permarrà sempre grande il fabbisogno di competenza e sensibilità culturale specifica negli internisti, anche perché, tenendo conto che solo il $25 \%$ del totale delle morti è dovuto al cancro, la tipologia dei malati potenziali beneficiari delle cure palliative sarà destinata ad essere rappresentata in maniera prevalente dalle insufficienze d'organo singole o multiple, gravi e irreversibili di squisita pertinenza internistica.

Bisogna ammettere che le cure di fine vita rappresentano una necessità non ancora pienamente soddisfatta in ospedale, soprattutto per la mancanza di formazione pre-laurea e post-laurea indirizzata in questo senso.

\section{Le cure di fine vita secondo i paradigmi della comfort medicine}

Il mondo degli internisti ospedalieri raccolto intorno alla FADOI, negli ultimi anni ha rivolto riflessioni ed iniziative alle cure di fine vita e alla medicina della terminalità, che hanno trovato articolazione in pubblicazioni monografiche dedicate, ${ }^{8,9}$ eventi pubblici come le Giornate Nazionali sulle Cure di Fine Vita in Medicina Interna istituite a partire dal 2018 in tutte le regioni italiane, e nella estensione di un Deca- logo FADOI-ANIMO sulle Cure di Fine Vita ${ }^{10}$ per i propri affiliati. Il Decalogo addita la necessità che gli internisti acquisiscano consapevolezza del problema (un gran numero dei decessi ospedalieri avvengono in Medicina Interna, ma non tutti gli internisti sono per questo già preparati, né tutti i reparti organizzati in maniera orientata) e che essi si riferiscano alle norme esistenti (Codice di deontologia medica 2016, Legge 219/17 sulle Disposizioni Anticipate di Trattamento, in particolare), indica le specifiche competenze professionali da acquisire ed accrescere da parte di ognuno (terapia del dolore, sedazione terminale, nutrizione e idratazione artificiale, tecniche comunicative), all'interno di principi umanistici ispiratori di fondo, che sono il rispetto della dignità personale del morente, la proporzionalità delle cure e la desistenza terapeutica, il ripudio di ogni idea di abbandono.

A grandi linee, le cure di fine vita secondo i paradigmi della comfort medicine all'interno di un reparto internistico contemplano: i) la identificazione dei pazienti idonei (non è la prima cosa che si deve fare all'ingresso in reparto, perché non si tratta mai di una decisione affrettata o urgente: questo va rimandato ai giorni successivi, per una decisione ponderata e condivisa); ii) la identificazione dei bisogni del paziente, ivi compresi quelli psicologici e spirituali; iii) la pianificazione assistenziale ad hoc (essenziale la cooperazione degli infermieri per le decisioni di loro pertinenza) che consideri la sospensione/non attivazione dei monitoraggi dei parametri vitali e la non attivazione di pratiche rianimatorie, ma anche la astensione dalle più routinarie pratiche infermieristiche di rilevazione dei parametri vitali non finalizzate al comfort del paziente; iv) la rimodulazione della terapia $^{11}$ (compresa quella considerata salva-vita) e la conversione alla via sottocutanea della terapia parenterale; v) la prescrizione della terapia anticipatoria per i sintomi disturbanti (dolore, dispnea, nausea/vomito, agitazione/delirium, rantolo terminale) e la terapia al bisogno per gli stessi; vi) la sorveglianza pro-attiva rivolta ai bisogni; vii) una comunicazione intensa e costante con i pazienti e i familiari, che va tracciata e documentata.

Queste cure si caratterizzano per le molte cose che intenzionalmente trascurano (esami di laboratorio, altri esami diagnostici, registrazione dei parametri vitali), che normalmente distolgono medici e infermieri da quello che veramente conta per il paziente, e si qualificano per l'atteggiamento proattivo sui bisogni, la sorveglianza sui sintomi disturbanti e il mantenimento di livelli di comunicazione non ordinari. Nessuna decisione è considerata definitiva e non rivedibile. Il tutto è rivolto al comfort del paziente.

Le cure di fine vita si caratterizzano per le molte cose che intenzionalmente trascurano 
(esami di laboratorio, altri esami diagnostici, registrazione dei parametri vitali), che distolgono medici e infermieri da quello che veramente conta per il paziente, e si qualificano per l'atteggiamento proattivo sui bisogni, la sorveglianza sui sintomi disturbanti e il mantenimento di livelli di comunicazione non ordinari.

\section{La diagnosi di morte imminente}

Il più delicato problema cui ci si trova davanti parlando di cure di fine vita, che intimidisce e scoraggia soprattutto i neofiti nell'affrontare un malato giunto al suo percorso finale, è quello del riconoscimento (vera e propria diagnosi) di morte imminente e in genere della possibilità di pronosticare il tempo residuo. Le domande cui si tratta esplicitamente o implicitamente di rispondere, in termini prosaici sono: qual è l'inizio della fine?, quanto tempo resta prima della morte?, quanto si è garantiti da possibili confutazioni da parte di colleghi o delle persone interessate?

Di fronte ad un malato cronico, neoplastico avanzato o con insufficienze d'organo gravi e irreversibili (insufficienza cardiaca, respiratoria, renale, epatica, demenza e altre sindromi neurologiche invalidanti), la diagnosi di morte imminente può fondarsi sui criteri validati dalla Liverpool Clinical Pathway for the Dying Patients: ${ }^{12}$ i) la non sussistenza della possibilità di intervenire sui fattori causali dello stato morboso attuale (deve essere chiaro, per esempio, che uno stato comatoso da ipercalcemia maligna in un paziente neoplastico va affrontata in prima istanza secondo i paradigmi della medicina per acuti correggendo la calcemia, qualsiasi sia la prognosi di fondo); ii) uno stato di allettamento cronico (per esempio, un Performance Status pre-ricovero $<50$, ciò che equivale ad uno stato di allettamento per la maggior parte delle ore diurne a domicilio); iii) alimentazione spontanea ridotta alla assunzione di pochi cibi liquidi; iv) incapacità nella assunzione di terapia p.o.; v) stato di persistente sonnolenza/sopore.

Il concetto di imminenza resta in un ambito di indeterminatezza sostanziale, non facilmente eliminabile. In uno studio recente ${ }^{13}$ condotto su 357 malati neoplastici avanzati è stata identificata una serie di segni presenti negli ultimi 3 giorni di vita con una specificità superiore al $95 \%$ nell'indicare la morte imminente (mancata reattività pupillare, diminuita risposta agli stimoli verbali e visivi, incapacità di chiudere le palpebre, iperestensione del collo, rantolo, emorragie gastroenteriche). Nel portare in evidenza un quadro molto eloquente, che non deve scomodare la statistica ma solo il buon senso clinico, lo studio ha confermato la incapacità di altri 44 segni presi in considerazione (compresi i normali parametri vitali, quali la $\mathrm{FC}$, la $\mathrm{PAO}$, la temperatura, gli atti respiratori e la $\mathrm{sO}$ ) di fornire indica- zioni predittive. A dire che, o sono presenti dei segni molto ovvii, o non si è in grado di anticipare alcunché, neppure in termini temporali molto stretti.

Una disamina sistematica degli indicatori per la definizione e la valutazione prognostica dei malati terminali o ad alto rischio di mortalità ospedaliera presenti in letteratura è stata condotta da Nardi e coll. per conto della FADOI nel 2015. ${ }^{14}$ La maggior parte degli indicatori della letteratura sono stati costruiti su casistiche oncologiche o su insufficienze d'organo singolarmente considerate, con risultati difficilmente trasferibili da una tipologia di malati ad un'altra. Negli anni successivi, per quanto riguarda lo specifico della Medicina Interna, sono stati elaborati indicatori originali $\left(\right.$ ACLAP $^{15}$, COMPLIMED $^{16}$ ), che sono venuti ad arricchire lo scenario. La pletora di indicatori e la continua ricerca in merito riflettono la difficoltà di arrivare a risultati soddisfacenti. Dunque, l'incertezza prognostica persiste, perché anche i migliori indicatori raggiungono una accuratezza dell'ordine del $70 \%$, poco utile per decisioni cliniche su singoli pazienti.

Nell'attesa di strumenti più raffinati, non ci si può rassegnare ad una paralisi decisionale, perché, se è vero che in medicina la autoreferenzialità va scongiurata e che è giusto basare le proprie scelte sulle evidenze, è comunque dovere del medico decidere al meglio, in coscienza, in nome della beneficialità.

Quanto alla confutabilità, bisogna dire che essa è intrinseca alle scelte mediche, perché solamente per una quota minoritaria del loro lavoro i medici si trovano ad agire all'interno di solide sponde di evidenze scientificamente fondate. Tanto è vero che su quasi ogni argomento si trovano raccomandazioni autorevoli per dare risposte ai quesiti clinici quotidiani. Ma le raccomandazioni non sono e non possono mai diventare prescrizioni garantiste: sono il punto di confluenza del pensiero scientifico su un certo argomento e strumenti di supporto alle scelte individualizzate, tanto più preziosi quanto più si collocano su terreni incerti. Se un medico cerca nelle linee guida il dettato delle sue scelte e la sicurezza psicologica, o, peggio, la protezione dai contenziosi, fa cattiva medicina, falsificando il senso stesso delle raccomandazioni delle linee guida (che servono ad indicare il modo in cui si entra in un problema, non il modo in cui se ne esce), e tradendo il proprio mandato per la medicina difensiva, quella che si fa a difesa di sé, non del malato. Quanto detto non deve aprire la strada a false sicurezze e alla autoreferenzialità, ma dare il coraggio e la capacità al medico di affrontare le vere incertezze, come tali.

Parlando delle scelte relative alle cure di fine vita, va sottolineato che è importante che all'interno di un reparto esse vengano maturate, organizzate e portate avanti come lavoro di equipe (coinvolgendo fin da subito la componente infermieristica), non come scelta 
individuale di un medico particolarmente sensibile al problema. Questo è fondamentale, non tanto per evitare le confutazioni e le messe in discussione, quanto le incoerenze dei comportamenti, e in ultima analisi l'efficacia delle scelte.

\section{Morire bene ed eutanasia}

Il termine eutanasia è equivoco. Per evitare malfondate controversie è importante chiarire di cosa si intende parlare.

Eutanasia (buona morte, in senso letterale) sembra a prima vista omologo e lessicalmente identico al concetto del morire bene, di cui siamo venuti parlando come diritto da garantire alle persone anziane. Ma il termine è usato per uno spettro di situazioni e significati che vanno distinti. ${ }^{17}$

Trascurando l'annientamento di vite indegne di vita propugnato dal nazionalsocialismo hitleriano (che pure veniva presentato come eutanasia), ad un estremo dello spettro si colloca la eutanasia attiva nella duplice accezione di morte su richiesta e suicidio assistito (nella prima il medico interviene direttamente, nella seconda assiste); queste pratiche sono legalizzate in alcuni paesi (Belgio, Olanda, Svizzera, e alcuni stati degli USA) entro limiti ben precisi. Al centro si colloca la eutanasia indiretta che fa riferimento al concetto del doppio effetto, esemplificato dall'uso di sedativi ad alte dosi con fini antalgici, potenzialmente capaci di accelerare decessi attraverso la depressione respiratoria (la reale consistenza di questo fenomeno è stata messa in discussione da studi che hanno mostrato come la sedazione terminale allunghi la vita residua, anziché accorciarla ${ }^{18}$ ). All'altro estremo si colloca l'eutanasia passiva nella accezione di desistenza terapeutica, che può passare da una deliberazione medica (a prescindere dalla volontà del paziente) o da una decisione volontaria di un paziente competente; ancora, la desistenza può riguardare la interruzione di terapie in atto (cfr. ventilazione meccanica), ovvero la rinuncia a priori di un trattamento: questa distinzione non coglie cose diverse dal punto di vista della rilevanza etico-giuridica, ma va tenuta presente per le sensibilmente diverse implicazioni psicologiche che riguardano chi è chiamato a decidere.

Le cure di fine vita e la comfort medicine non hanno nulla a che fare con la eutanasia attiva (morte su richiesta, suicidio assistito), ed anzi se ne discostano sostanzialmente: le prime sono una forma di accanito accompagnamento del paziente morente rivolto al lenimento dei sintomi disturbanti, con attenzione alla dimensione psicologica e spirituale della assistenza, lasciando che la morte succeda, a suo tempo; la seconda è un intervento tecnicamente molto preciso e puntuale, che irrompe nel dominio del tempo (hic et nunc), rivolto a generare un evento (la morte).
La desistenza terapeutica non è mai rinuncia per se stessa, ma scegliere il meglio per il paziente (possibilmente insieme con lui), e quindi semplicemente buona medicina.

La legislazione italiana non legittima la eutanasia attiva; il codice deontologico medico la ripudia.

Il Decalogo FADOI-ANIMO sulle Cure di Fine Vita si colloca in linea con la deontologia medica (non avrebbe senso che ne derogasse), e asserisce che non iniziare dei trattamenti o sospenderli, se essi non rispondono a criteri etici e umanistici di proporzionalità delle cure, non significa causare la morte, ma accettare che essa non possa essere prevenuta.

Le cure di fine vita non hanno nulla a che fare con la eutanasia attiva (morte su richiesta, suicidio assistito), ed anzi se ne discostano sostanzialmente: le prime sono una forma di accanito accompagnamento del paziente morente, e lasciano che la morte succeda, a suo tempo; la seconda è un intervento puntuale, che irrompe nel dominio del tempo (hic et nunc), rivolto a generare un evento (la morte).

Il Decalogo FADOI-ANIMO sulle Cure di Fine Vita asserisce che "non iniziare dei trattamenti o sospenderli, se essi non rispondono a criteri etici e umanistici di proporzionalità delle cure, non significa causare la morte, ma accettare che essa non possa essere prevenuta'.

\section{Conclusioni}

Queste precisazioni sulla eutanasia e il morire bene, enunciate in maniera apodittica, non assorbono né esauriscono gli argomenti che sottostanno alle discussioni di ordine etico che entrano in scena ogni volta che ci si pone di fronte al problema della morte e del suo senso. Sono qui poste ai fini di favorire ogni discussione, ma soprattutto di evitare che, di fronte alla consistenza del problema, il medico internista si senta sovrastato, e che questo sentimento dia adito ad alibi per evitare di cambiare comportamenti e scelte abitudinarie. In realtà parlando di malati anziani dentro traiettorie di malattie croniche allo stadio di gravità irreversibile, non possiamo proiettare l'universo delle problematiche legate alla vita e alla morte (diverso è pensare ai nascituri, ai bambini, ai giovani traumatizzati, agli stati vegetativi, alle persone mantenute in vita con mezzi artificiali). Il fine della vita negli anziani va affrontato con naturalezza e in riferimento alla naturalità del morire, e soprattutto con la semplice preoccupazione di non diventare artefici, al di là di ogni intenzione, di sofferenze aggiuntive per i morenti.

La medicina nella quale siamo immessi ha un moto inerziale impresso da un imperativo tecnologico che, purtroppo, va in direzione contraria alle esigenze 
umanistiche basilari delle persone, e richiede efficienze che comprimono fino a sottrarre del tutto il tempo della riflessione condivisa, della comunicazione e della relazione nella cura.

Tutti ne sono vittime. Ai medici, e agli internisti in particolare, il dovere di non rendersene complici.

\section{Il fine della vita negli anziani va affrontato} con naturalezza e in riferimento alla naturalità del morire, e soprattutto con la semplice preoccupazione di non diventare artefici, al di là di ogni intenzione, di sofferenze aggiuntive per $i$ morenti.

\section{Bibliografia}

1. ÒConnell M. Essere una macchina. Milano: Adelphi; 2018.

2. Harari YN. Homo deus. Milano: Bompiani; 2018.

3. Gawande A. Being mortal. London: Wellcome Collection; 2017.

4. Blinderman CD, Billings AB. Comfort care for patients dying in the hospital. N Engl J Med 2015;373:2449-61.

5. Lusiani L, Bordin M, Mantineo G, et al. Cure di fine vita nei pazienti oncologici terminali in Medicina Interna. Ital J Med 2012;6:110-5.

6 . Beccaro M, Costantini M, Giorgi Rossi P, et al. Acutal and preferred place of death of cancer patients. Results from the Italian survey of the dying of cancer (ISDOC). J Epidemiol Community Health 2006;60:412-6.

7. Bertolissi S, Miccinesi G, Giusti F. Come si muore in Italia. Storia e risultati dello studio Senti-MELC. Rivista SIMG 2013;2:17-34.

8. Zulian F, Campanini M, Lusiani L, et al. vari. Proble- matiche di fine vita: il ruolo della Medicina Interna. Quaderni Ital J Med 2017;5:1-146.

9. Zulian F, Campanini M, Lusiani L, et al. Saper desistere per curare fino in fondo: come intraprendere il migliore percorso di assistenza nel paziente con grave insufficienza d'organo in Medicina Interna. Quaderni Ital J Med 2017;5:1-148.

10. Lusiani L, Ballardini G, Nardi R, et al. A decalogue for end-of-life care in Internal Medicine. Ital $\mathrm{J}$ Med 2018;12:230-4.

11. Cester A, Busonera F. La rimodulazione della terapia negli anziani in fase terminale. Quaderni Ital J Med 2017;5:85-92.

12. Ellershaw J, Foster A, Murphy D, et al. Care for the dying: the last hours or days of life. BMJ 2003;326:30-4.

13. Hui D, Dos Santos R, Chisholm G, et al. Bedside clinical signs associated with impending death in patients with advanced cancer: preliminary findings of a perspective, longitudinal cohort study. Cancer 2015;121: 960-7.

14. Nardi R, Belmonte G, Lusiani L, et al. Metodi di riconoscimento e di valutazione del paziente in fase terminale o a rischio di elevata mortalità in ospedale. Quaderni Ital J Med 2017;5:111-45.

15. Tiraboschi M, Ghidoni S, Zucchi A, et al. I determinanti della mortalità a breve termine dei pazienti non oncologici ricoverati in Medicina Interna: un'esperienza sul campo. Quaderni Ital J Med 2017;5:40-8.

16. Bonizzoli E, Gussoni G, Anelli G, et al. The complexity of patients hospitalized in Internal Medicine wards evaluated by FADOI-COMPLIMED score. A hypothetycal approach. PLoS One 2018 [Epub ahead of print].

17. Borasio GD. Saper morire. Torino: Bollati-Boringhieri; 2015.

18. Kohara H, Ueoka H. Sedation for terminally ill patients with cancer with uncontrollable physical distress. J Palliat Med 2005;8:4-20. 


\section{Il programma delle otto $R$ : utopia o possibile realtà per migliorare l'assistenza agli anziani?}

Francesco D'Amore

Primario f.r. di Medicina Interna, Roma, Italia

\section{L'angoscia della vecchiaia}

Il sentimento e l'entusiasmo ch'era il compagno e l'alimento della mia vita, è dileguato per me in un modo che mi raccapriccia.

Giacomo Leopardi esprime in questo modo, nel contesto di una lettera all'amico Pietro Brighenti, le sensazioni dell'energia vitale che lo sta abbandonando. ${ }^{1}$

Leopardi non era certamente vecchio quando scrive, con straordinaria sensibilità, questa frase che può far sua un uomo anziano che sente ridurre le proprie energie ed avverte quasi una dissociazione tra il progressivo indebolimento del corpo e i desideri non ancora sopiti dell'animo, una parabola che conduce ad un destino senza orizzonti.

L'uomo prende coscienza della propria fragilità e si fa strada la sensazione di un futuro incerto nel quale non hanno più posto progetti di lavoro, di relazioni, di affetti, un futuro di cui non è più protagonista. Affiorano domande che hanno il colore dell'angoscia e della depressione. Perché vivere a lungo? A chi e a che cosa serve? Qual è il significato della vita? Ritornano inesorabili i ricordi della vita passata ed il senso di immortalità che pervade la società moderna, lascia il posto al pensiero della morte.

Filosofi, poeti, scrittori di tutti i tempi hanno fatto grandi affreschi della vecchiaia, straordinarie e mirabili descrizioni dell'ultimo tratto della vita ed hanno cercato di dare interpretazioni, rimedi e consigli ma si riferivano ad una popolazione percentualmente poco rappresentata e della quale se ne faceva carico, per la quasi totalità, la famiglia.

Corrispondente: Francesco D’Amore, Primario f.r. di Medicina Interna, via Rodolfo Lanciani 2, 00162 Roma, Italia. Fax: +39.06.3729885

E-mail: dott_damore@libero.it

Articolo pubblicato secondo la Creative Commons Attribution NonCommercial 4.0 License (CC BY-NC 4.0).

CCopyright F. D'Amore, 2019

Licensee PAGEPress, Italy

QUADERNI - Italian Journal of Medicine 2019; 7(4):75-78
Nella società di qualche tempo fa, l'anziano, sia pure fragile e vulnerabile, era rispettato ed ascoltato per l'esperienza e la saggezza accumulate ma, nel mondo di oggi, in cui la velocità brucia ogni cosa, usa e getta, è ancora tollerata la lentezza dell'anziano? Il suo pensare, il suo muoversi, il suo parlare, la sua inesperienza nel padroneggiare gli strumenti tecnologici?

Come agire allora, quando il peso sociale, economico e familiare della popolazione ultrasettantenne italiana, che sfiora il $20 \%$ di quella totale, si riflette in tutta la sua complessità sulla società?

\section{L'ossessione della crescita}

Nel mondo economico della società occidentale $\mathrm{e}$, più in generale, nelle nazioni del Nord del pianeta da quasi un secolo rimbalza l'eco inesauribile della crescita, crescita, crescita. L'economia ha fatto della crescita il suo dogma e, per dare concretezza e possibilità alla produzione industriale di sviluppare volumi sempre maggiori, ha stimolato un consumismo compulsivo prodotto da nuove necessità e bisogni. Una società della crescita che ha la sua forza in una produzione industriale che non deve avere pause o cedimenti e che ha il suo braccio armato nell'idea della obsolescenza, di cui se ne possono distinguere tre tipi: i) obsolescenza tecnica, ossia il prodotto è superato da un altro prodotto effettivamente più efficace e più economico; ii) obsolescenza psicologica, una martellante campagna pubblicitaria propone il superamento di un articolo, peraltro ancora del tutto valido; iii) obsolescenza programmata, ovvero produzione di strumenti costruiti con componenti con un tempo limitato di efficacia, pianificato tecnicamente.

Produzione, consumo, obsolescenza costituiscono tre pilastri del mondo globalizzato che ha acceso speranze e migliorato condizioni di vita ma ha anche creato profonde disuguaglianze economiche e culturali e forti tensioni sociali.

In questo sistema non può essere dimenticata, anzi ne è il cuore pulsante, la ricerca che ha portato straordinarie conoscenze in tutti i campi, producendo strumenti dotati di una tecnologia sempre più sofisticata e che determinerà, in un futuro non lontano, sostan- 
ziali modificazioni nel campo lavorativo ed un'amplificazione delle capacità intellettive e fisiche dell'uomo attraverso gli strumenti dell'intelligenza artificiale.

Hannah Arendt ${ }^{2}$ scrive la nostra intera economia è divenuta un'economia di spreco, in cui le cose devono essere divorate ed eliminate con la stessa rapidità con cui sono state prodotte, ammesso che il processo stesso non giunga a una fine improvvisa e catastrofica.

Un modello economico e sociale che appare consolidato e che fa pensare e temere, per alcuni aspetti, ad un esaurimento di risorse primarie, al consumo della terra e delle sue bellezze, in sostanza ad una perdita di valori non rinnovabili. Lo smarrimento in cui l'umanità potrebbe venire a trovarsi, ha fatto nascere riflessioni sui comportamenti della società occidentale introducendo pensieri diversi sul concetto di benessere ed in particolare sull'uomo, considerato come una macchina revisionabile in tutti i suoi pezzi.

Alcuni avvenimenti, sempre più frequenti negli ultimi anni, come i cambiamenti climatici caratterizzati da manifestazioni improvvise e catastrofiche, la linea della desertificazione che si sposta continuamente più a Nord, la riduzione delle calotte polari e dei ghiacciai ad ogni latitudine, i problemi legati alla produzione agricola ed alla pesca, hanno posto, non solo a livello nazionale, ma anche nell'agenda delle grandi assise internazionali, l'urgente necessità di attuare provvedimenti rispettosi della natura, del clima, dell'ambiente. A questo fine sono stati proposti, tra molte contrastanti idee dei rappresentanti delle nazioni, progetti per limitare i danni provocati dall'industria e dal consumo del suolo, per uno sviluppo controllato dell'agricoltura e per un razionale comportamento alimentare consigliato dal movimento dello slow food in opposizione alla moda del fast food e dello junk food.

\section{L'utopia della decrescita}

Personaggi come Thoreau, Ruskin, Tolstoj contestarono, fin dal nascere della rivoluzione industriale, nella seconda metà dell'ottocento, il consumismo senza freni e senza limiti, perché immaginavano una società attenta ai bisogni reali dell'uomo, non condizionata dalla pubblicità, impegnata alla costruzione di una società più giusta e più semplice (simple living, downshift, simplicity with style), semplicità che, negli U.S.A., si ispira alla filosofia di Henry Thoreau.

Dopo un lungo periodo di relativo silenzio, negli anni sessanta del secolo scorso, il movimento, che prende il nome di decrescita (termine entrato solo da pochi decenni nel nostro vocabolario), trova la forza di lanciare un'offensiva importante ad opera di John Kennet Golbraith e Vance Packard. Col termine decrescita si intendono idee e relativi comportamenti che oppongono alla crescita illimitata e al consumismo senza freni, la costruzione di una società con uno stile di vita frugale, sobrio, semplice. La decrescita non vuole essere una forma di recessione, di regressione o di crescita zero ma piuttosto un progetto per una politica del doposviluppo (Serge Latouche).

Sono trascorsi da allora alcuni decenni ma i titoli della comunicazione riguardano ancora, tutti i giorni, i numeri dell'economia ed in particolare quelli dell'aumento o della riduzione della crescita. La decrescita è una parola d'ordine, uno slogan per contrastare la crescita per la crescita e che indica, invece, la strada di un'interpretazione e programmazione della vita umana nella quale dovrebbero avere meno spazio i sovra, sovrasviluppo, sovrapproduzione, sovrainquinamento, sovraconsumo, sovrabbondanza, sovramedicalizzazione, come scrive Jean-Paul Besset. ${ }^{3}$ Besset aggiunge e sottolinea Uscire dall'autostrada del progresso non significa chiudersi nel vicolo cieco del passato.

L'impegno di coloro che teorizzano questi concetti, tende alla realizzazione di un sogno, una società più libera da condizionamenti, più sobria, più felice $\mathrm{e}$ in grado, quindi, di rifiutare il superfluo, quello che soffoca ed opprime, quello che è imposto dalle mode e dalle convenzioni. ${ }^{4}$

La decrescita così teorizzata non sembra rappresentare un modello che possa sostituirsi a quello dello sviluppo, ma ha in sé valori che possono generare comportamenti validi ed efficaci per l'uomo e per l'ambiente.

Lo sviluppo sostenibile è quello che soddisfa le necessità delle attuali generazioni senza compromettere la capacità delle future generazioni di soddisfare le proprie (Commissione mondiale sull'ambiente e lo sviluppo dell'ONU, 1987).

\section{Il programma delle otto $R$}

Serge Latouche, economista francese, in questa visione, ha proposto la possibilità della costruzione di un mondo della decrescita serena attraverso il programma radicale, sistematico, ambizioso delle otto $R{ }^{5}$ Otto $R$ che costituiscono un circolo virtuoso che inizia da Rivalutare, cioè ripensare ad un cambiamento dei bisogni e dello stile di vita, e nel quale potrebbe entrare, come suggerisce Roberto Nardi, un'altra R, Ridimensionare, che, meglio di tante altre $\mathrm{R}$, caratterizzerebbe la società della sobrietà; organizzare, cioè, su basi nuove, per adeguarsi a nuove esigenze.

Le otto $R$ proposte da Latouche sono: i) Rivalutare: rivoluzione culturale per uscire dalla religione dell'eccesso; ii) Riconcettualizzare: educazione e formazione, ripensare la costruzione dei cittadini; iii) $R i$ ciclare: lavorare i rifiuti delle nostre attività; iv) Ristrutturare: adattare la produzione ai valori; v) Riutilizzare: imparare a riparare e a comprare prodotti di seconda mano; vi) Ridistribuire: ripartire le ricchezze 
ed il patrimonio della natura; vii) Ridurre: ridimensionare i bisogni e lo stile di vita; viii) Rilocalizzare: pensare globalmente, agire localmente.

Il modello di un mondo più semplice, così concepito, potrebbe essere la base teorica della sanità italiana per costruire un sistema rivolto alla popolazione anziana che ha necessità di essere assistita, non solo per il peso delle malattie organiche, ma anche e soprattutto per quello prodotto dai problemi psicologici. Non si può pensare ad una ricetta unica, valida per ogni anziano, ma certamente il senso insito nella $R$ del Ridimensionare porterebbe a immaginare una rete che intercetti i bisogni comuni, una rete capace di promuovere servizi e programmi capaci di rendere la vita del malato anziano più facile. Di conseguenza dovrebbero essere rivisti i percorsi di un uomo che non ha più l'energia di attendere ore per essere visitato al Pronto Soccorso, che non è nelle condizioni di restare su una barella spesso per giorni, che non è pronto a rispondere velocemente alle domande di un medico ospedaliero, sempre diverso, in occasione di una visita ambulatoriale. Una situazione che, nella realtà quotidiana, non rispetta la dignità dell'uomo che appare subordinata a tante altre esigenze.

Il programma delle $R$ di Latouche ha probabilmente un sapore utopico, ma prospetta la possibilità di una condizione di vita coerente per un uomo più fragile e vulnerabile. La proposta di Latouche allontana il pericolo adombrato da Alexis de Tocqueville ${ }^{6}$ che aveva prospettato una obsolescenza dell'onore, obsolescenza dell'uomo stesso, e che immagina una società che non rivolge lo sguardo all'indietro ma ha l'obiettivo di rispondere ai bisogni ed ai valori di ciascuna persona con umanità, senza assumere, peraltro, una forma romantica.

\section{Un'idea nuova di assistenza}

Nessuna ricerca può negare i vantaggi che lo sviluppo ha messo a disposizione dell'uomo aiutandolo nelle invalidità, riducendogli i disagi, accompagnandolo a superare le complicazioni che si possono verificare nei lunghi anni di sofferenza causate da malattie croniche.

La qualità dell'assistenza agli anziani, pur tuttavia, non sempre e dappertutto è adeguata: difficoltà di accedere alle cure, tortuosità burocratiche, tempi infiniti per l'esecuzione di esami, disagi di ogni tipo in caso di ricovero, assenza di strutture o comunità che possano diminuire l'angoscia della solitudine, vera e propria malattia della vecchiaia.

Si dovrebbe auspicare, quindi, il rinnovamento di una programmazione comprendente servizi dedicati alla manutenzione continua nel tempo, all'implementazione di interventi routinari, domiciliari, medici e infermieristici, ripetuti e flessibili. La grave carenza che si registra oggi, infatti, non riguarda le situazioni urgenti che vengono assolte in modo straordinariamente tempestivo ed efficace, ma quella che si dovrebbe occupare dell'anziano con i suoi bisogni cronici quotidiani.

Un tale sistema intercetterebbe tante potenziali condizioni di instabilità clinica, eviterebbe l'esorbitante numero degli accessi ai Pronto Soccorso e dei re-ricoveri, migliorerebbe l'aderenza alla terapia e darebbe alle persone la tranquillità di un'assistenza che pone al centro il singolo malato.

Il programma di Latouche potrebbe facilitare, in effetti, un'attività assistenziale personalizzata perché ha in sé lo stimolo di provocare una riflessione sulla istruzione e formazione specifica del personale sanitario, sulla sua più razionale distribuzione, sulla ricollocazione delle risorse economiche, sulla necessaria strumentazione tecnologica per un aiuto umanizzato.

Le $R$ di Latouche sembrano formare la traccia di un percorso e, contestualmente, rappresentare un feedback di verifica relativa alla validità del piano, elaborato per ogni singola persona, per andare incontro non solo alle sue specifiche esigenze sanitarie ma anche, come ripetutamente sottolineato, per compensare i vuoti familiari e amicali.

Ogni $R$, rivalutare, riconcettualizzare, ristrutturare, ridistribuire, rilocalizzare, ridurre, riutilizzare, riciclare, spinge, infatti, a esaminare tutti gli aspetti che riguardano l'attività dell'uomo e a prenderne le relative misure; un lavoro complesso che dovrebbe impegnare, ovviamente, non solo la sanità ma tante e diverse strutture, sociali e civili, che potrebbero far liberare talenti sopiti, utili al singolo e alla collettività.

Si renderebbe manifesto, in tal modo, non solo quello che la società fa per l'anziano ma anche quello che questi potrebbe fare per la società.

Una comunità, nella quale dovrebbero prevalere azioni di vicinanza e comportamenti clinici ragionati, la Slow medicine e la Choosing wisely troverebbero terreno fertile per le loro raccomandazioni: riportare al loro giusto valore i fondamentali dell'esame clinico, rispondere a criteri di appropriatezza esami, procedure, consulti, personalizzare i trattamenti terapeutici condivisi in un'alleanza terapeutica, evitare di sperperare risorse per eseguire prestazioni inutili e spesso dannose. $^{7}$

L'auspicabile umanesimo della decrescita potrebbe convivere con i totem della modernità e del progresso per attuare le migliori forme comportamentali e valoriali, adeguate ad una comunità di anziani, antichi gesti consolatori, volti ad esorcizzare la tristezza, esilio dei vecchi, come immaginava Simone de Beauvoir.

La vecchiaia cambia il modo di vivere, ma non deve essere il tempo di un ripiegamento su se stessi, anzi, deve continuare ad esplorare sentimenti ed affetti con una nuova consapevolezza, con un senso di curio- 
sità che non invecchia, scrive lo psichiatra Massimo Ammaniti. $^{8}$

L'uomo non è un assemblaggio, per quanto complesso, di pezzi ma un unico del quale prendersi cura in una visione globale ed è proprio affermando questo concetto che il programma di Latouche assume una sua realistica validità poiché concorrerebbe ad allargare l'orizzonte di un domani assistenziale più socializzante, più dialogante, più felice.

\section{Bibliografia}

1. Leopardi G. Lettera a Pietro Brighenti, 21 aprile 1820.

2. Arendt H. Vita activa. La condizione umana (1958). Milano: Bompiani; 2004. p. 95.

3. Besset J-P. La scelta difficile. Come salvarsi dal progresso senza essere reazionari. Bari: Dedalo; 2007.

4. Toraldo di Francia G. Il Rifiuto. Torino: Einaudi; 1978.

5. Latouche S. La scommessa della decrescita. Milano: Feltrinelli; 2016.

6. Chanial P. La Sociologie comme philosophie politique et réciproquement. Paris: La Découverte; 2011.

7. Frediani R. Choosing Wisely, le scelte di FADOI, Bertinoro 17-18 novembre 2017.

8. Ammaniti M. La curiosità non invecchia. Milano: Mondadori; 2017. 


\section{LINEE GUIDA PER GLI AUTORI}

I Quaderni dell 'Italian Journal of Medicine (Quaderni ITJM), costituiscono una collana supplementare solo online annessa alla rivista Italian Journal of Medicine contenente lavori solo in lingua italiana.

I Quaderni ITJM pubblicano:

- Monografie ad hoc individuate dal Presidente FADOI, dal Consiglio Direttivo, dal Board Scientifico o dall'Editor in Chief dell'Italian Journal of Medicine, in funzione del contesto scientifico-istituzionale attuale.

- Monografie ad hoc su temi di particolare rilevanza scientifica a cura della Commissione FADOI Giovani.

- Traduzioni in italiano di alcuni lavori pubblicati sui numeri standard dell'Italian Journal of Medicine, di particolare interesse per la comunità scientifica.

\section{STESURA DEI LAVORI}

I lavori dovranno essere redatti in modo conforme alle linee guida sotto riportate:

- I manoscritti devono essere scritti interamente in lingua italiana, su documento di Word, con carattere Times New Roman/Arial, dimensione 12, formato A4, interlinea doppia e margini $2,54 \mathrm{~cm}$

Parole totali: $\max$ 4000; Sommario/Abstract: $\max 250$ parole; Bibliografia: min 40 voci; Tabelle e Figure: $3 / 5$ totali (le tabelle non devono superare n. 1 pagina del documento in Word).

- La strutturazione del contenuto deve attenersi agli standard internazionali per la Rassegna (Review): i) Abstract riassuntivo dell'intero lavoro; ii) Introduzione al tema trattato; iii) Criteri e strumenti di ricerca (criteri di inclusione/esclusione, banche dati consultate, ...); iv) i successivi paragrafi devono illustrare le più recenti scoperte scientifiche nel settore; v) Conclusioni; vi) Bibliografia.

- La prima pagina deve riportare: i) titolo (in stampatello minuscolo), senza acronimi; ii) nome e cognome per esteso di ciascun autore; iii) affiliazione(i) di ciascun autore, numerate con numeri arabi iv) eventuali ringraziamenti; v) nome e indirizzo postale completi dell'autore corrispondente, corredati da telefono, fax, e-mail; vi) da 3 a 5 parole chiave, separate da virgola. La seconda pagina può riportare: i) contributi degli autori, e.g. informazioni relative a contributi sostanziali delle persone coinvolte nello studio (http://www.icmje.org/\#author); ii) dichiarazione relativa a potenziali conflitti d'interesse; iii) ulteriori informazioni (e.g. fondi, esposizioni durante conferenze...).

- In caso di utilizzo di tabelle, queste devono essere tutte numerate con numeri arabi e citate nel testo in ordine consecutivo (e.g. NON nominare le tabelle come Tabella $1 \mathrm{~A}, 1 \mathrm{~B}, \ldots$ o $1.0,1.1, \ldots$ ). Le tabelle devono essere presentate in formato editabile. Ciascuna tabella deve essere corredata da una breve didascalia; in caso di abbreviazioni, riportare una nota a piè di CIASCUNA tabella che spieghi TUTTE le abbreviazioni presenti in ognuna.

- In caso di utilizzo di figure, queste devono essere inviate in formato tiff o .jpg, allegate al manoscritto in singoli files, secondo le seguenti specifiche:

i) a colori (salvate in modalità CMYK): minimo 300 dpi di risoluzione;

ii) in bianco e nero: minimo $600 \mathrm{dpi}$ di risoluzione;

iii) minimo $17,5 \mathrm{~cm}$ di larghezza.

Ciascuna figura deve essere corredata da una breve didascalia.

$N B$ : In caso di Tabelle/Figure riprese e/o modificate da altri lavori già pubblicati, sarà cura degli autori accertarsi se tali materiali siano o meno coperti da copyright e procurarsi i permessi necessari per la riproduzione. Tali permessi dovranno essere allegati alla versione definitiva del lavoro. L'ufficio editoriale si riserva la facoltà di rimuovere Tabelle/Figure coperte da copyright, se sprovviste dei necessari permessi.

- In caso di utilizzo di abbreviazioni, la prima volta che esse sono citate è necessario scrivere per esteso la definizione+abbreviazione tra parentesi tonde [e.g. risonanza magnetica $(\mathrm{RMN})]$, a seguire si dovrà riportare solo l'abbreviazione (unica eccezione: nei titoli e nelle didascalie di tabelle e figure NON si utilizzano abbreviazioni).

\section{BIBLIOGRAFIA}

Le voci bibliografiche devono essere formattate secondo lo stile Vancouver.

Nella sezione Bibliografia, le voci bibliografiche devono essere numerate consecutivamente nell'ordine in cui appaiono per la prima volta nel testo (NON in ordine alfabetico) e, nel testo, devono essere indicate con numeri arabi in apice. Voci bibliografiche riferite a comunicazioni personali o dati non pubblicati devono essere incorporate nel testo e NON inserite tra le voci numerate [e.g. (Wright 2011, dati non pubblicati) o (Wright 2011, comunicazione personale)]. Le voci bibliografiche nella sezione Bibliografia devono tassativamente essere preparate come segue:

i) più di 3 autori, citare 3 autori, et al. Se il lavoro contiene solo 4 autori, citarli tutti e 4 ;

ii) titolo del lavoro in stampatello minuscolo;

iii) nome della rivista, senza punti, abbreviato secondo gli standard internazionali; in caso di dubbi sulla corretta abbreviazione, fare riferimento ai seguenti siti:

a. ISI Journal Abbreviations Index (http://library.caltech.edu/ reference/abbreviations/);

b. Biological Journals and Abbreviations (http://home.ncifcrf. gov/research/bja/);

c. Medline List of Journal Titles (ftp://ftp.ncbi.nih.gov/pubmed/J_Medline.txt);

iv) inserire l'anno di pubblicazione subito dopo il nome della rivista, seguito da punto e virgola;

v) NON inserire giorno o mese di pubblicazione;

vi) citare solo il volume, seguito dai due punti (NON citare il fascicolo tra parentesi);

vii) abbreviare le pagine, e.g. 351-8

Per accertarsi di aver correttamente formattato le voci bibliografiche, confrontarle con le citazioni in PubMed (http://www.ncbi. nlm.nih.gov/pubmed).

Esempi (prestare attenzione anche alla punteggiatura):

Articolo standard su Rivista

Halpern SD, Ubel PA, Caplan AL. Solid-organ transplantation in HIV-infected patients. N Engl J Med 2002;347:284-7.

Proceedings

Christensen S, Oppacher F. An analysis of Koza's computational effort statistic for genetic programming. In: Foster JA, Lutton E, Miller J, Ryan C, Tettamanzi AG, eds. Genetic programming. EuroGP 2002: Proceedings of the 5th European Conference on Genetic Programming, 2002 Apr 3-5, Kinsdale, Ireland. Berlin: Springer; 2002. pp 182-91.

Articoli i cui autori sono Organizzazioni

Diabetes Prevention Program Research Group. Hypertension, insulin, and proinsulin in participants with impaired glucose tolerance. Hypertension 2002;40:679-86.

Libri

Murray PR, Rosenthal KS, Kobayashi GS, Pfaller MA. Medical microbiology. 4th ed. St. Louis, MO: Mosby; 2002. (CITAZIONE DEL LIBRO INTERO)

Meltzer PS, Kallioniemi A, Trent JM. Chromosome alterations in human solid tumors. In: Vogelstein B, Kinzler KW, eds. The genetic basis of human cancer. New York, NY: McGraw-Hill; 2002. pp 93113. (CITAZIONE DI UN CAPITOLO)

\section{MODALITÀ D'INVIO DEI LAVORI}

Monografie

Gli Autori dovranno fare riferimento all'Editor-in-Chief o alle persone da lui designate nelle lettere di invito a scrivere gli articoli programmati.

Per gli articoli inviati da giovani internisti, gli Autori dovranno fare riferimento alla Dr.ssa Paola Gnerre (e-mail: pgnerre@yahoo.it) e agli eventuali altri referenti da lei designati, nelle prime fasi di stesura dei manoscritti, revisioni e correzioni. 


\section{QUADERNI}

dell'Italian Journal

of Medicine

La Dr.ssa Gnerre raccoglierà poi le versioni definitive dei lavori di ciascuna monografia e provvederà all'invio di tutti i materiali all'ufficio editoriale.

I lavori solo nella loro versione definitiva e approvata dalla Commissione FADOI Giovani dovranno pervenire all'ufficio editoriale già pronti per l'impaginazione e immediata pubblicazione (già corredati da eventuali permessi per la riproduzione di tabelle e immagini redatti secondo le presenti linee guida).

Traduzioni

Previo invito dell'Editor-in-Chief, gli Autori dovranno far pervenire all'ufficio editoriale la versione tradotta in italiano, al seguente indirizzo e-mail: paola.granata@pagepress.org

Il file in formato Word dovrà essere formattato secondo gli standard editoriali della rivista ufficiale ed essere già pronto per impagina- zione e immediata pubblicazione (corredato da eventuali permessi per la riproduzione di tabelle e immagini).

Si prega di inviare le eventuali tabelle in formato editabile e le figure in alta definizione secondo gli standard sopra riportati.

\section{NOTA PER GLI AUTORI}

I lavori pubblicati sui Quaderni ITJM non verranno indicizzati, ma saranno liberamente disponibili in un'apposita sezione del sito FADOI (http://www.fadoi.org/) e della rivista ufficiale.

Gli Autori i cui lavori siano accettati per la pubblicazione sui Quaderni ITJM e che fossero interessati a vederli pubblicati anche sulla rivista ufficiale, dovranno sottomettere attraverso il sito dell'ITJM (www.italjmed.org) la versione (già tradotta) in inglese e redatta in modo conforme alle linee guida della rivista; seguiranno poi la procedura di selezione tramite peer review e, se accettati, saranno inseriti nel piano editoriale standard.

\section{STAFF EDITORIALE}

Paola Granata, Journal Manager

paola.granata@pagepress.org

Claudia Castellano, Production Editor

Tiziano Taccini, Technical Support
PUBBLICATO DA

PAGEPress Publications

via A. Cavagna Sangiuliani 5

27100 Pavia, Italy

T. +39.0382 .464340$

F: +39.0382 .34872$

\section{agepress}

www.pagepress.org info@pagepress.org

\section{QUADERNI - ITALIAN JOURNAL OF MEDICINE}

Tutti gli articoli pubblicati sui QUADERNI - Italian Journal of Medicine sono redatti sotto la responsabilità degli Autori. La pubblicazione o la ristampa degli articoli della rivista deve essere autorizzata per iscritto dall'editore. Ai sensi dell'art. 13 del D.Lgs 196/03, i dati di tutti i lettori saranno trattati sia manualmente, sia con strumenti informatici e saranno utilizzati per l'invio di questa e di altre pubblicazioni e di materiale informativo e promozionale. Le modalità di trattamento saranno conformi a quanto previsto dall'art. 11 del D.Lgs 196/03. I dati potranno essere comunicati a soggetti con i quali PAGEPress intrattiene rapporti contrattuali necessari per l'invio delle copie della rivista. Il titolare del trattamento dei dati è PAGEPress Srl, via A. Cavagna Sangiuliani $5-27100$ Pavia, al quale il lettore si potrà rivolgere per chiedere l'aggiornamento, l'integrazione, la cancellazione e ogni altra operazione di cui all'art. 7 del D.Lgs 196/03.

https://www.italjmed.org/index.php/ijm/quad 
PAGEPress - Scientific Publications

Via A. Cavagna Sangiuliani, 5 - 27100 Pavia - Italy

Phone +390382 464340 - Fax +390382 34872

www.pagepress.org 